\author{
ZENTRUM \\ FÜR BIODIVERSITÄT UND NACHHALTIGE LANDNUTZUNG \\ SEKTION \\ BIODIVERSITÄT, ÖKOLOGIE UND NATURSCHUTZ \\ - Centre of Biodiversity and Sustainable Land Use - \\ SEction: Biodiversity, Ecology and Nature Conservation
}

\title{
Assembly processes in soil animal communities: Integrating phylogeny and trait-based approaches
}

\author{
Dissertation zur Erlangung des Doktorgrades der \\ Mathematisch-Naturwissenschaftlichen Fakultäten der \\ Georg-August-Universität Göttingen
}

\author{
vorgelegt von \\ Master of Science \\ Ting-Wen Chen \\ aus \\ Kaohsiung City, Taiwan
}

Göttingen, Dezember 2017 

Referent:

Prof. Dr. Stefan Scheu

Korreferent:

Prof. Dr. Holger Kreft

Tag der mündlichen Prüfung: $\quad$ 26.01.2018 

Nothing in biology makes sense except in the light of evolution.

Theodosius Dobzhansky 



\section{Table of contents}

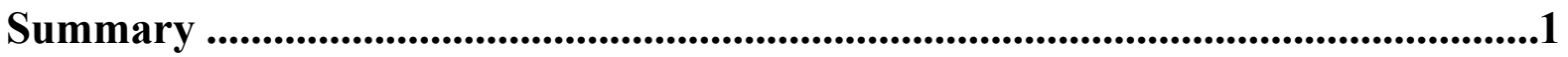

\section{Chapter 1}

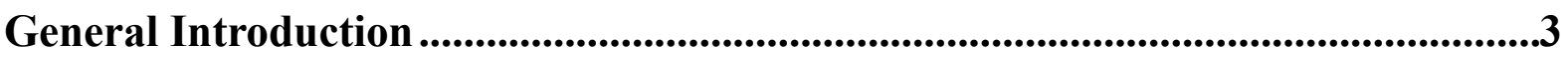

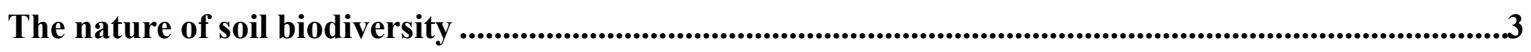

Inferring processes from patterns ...........................................................................................................3

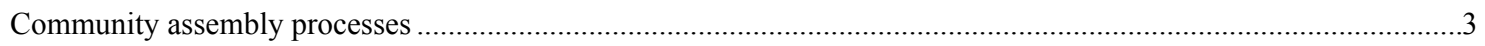

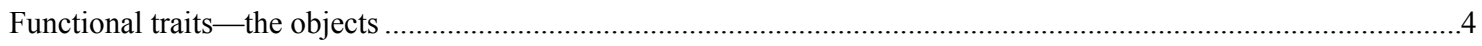

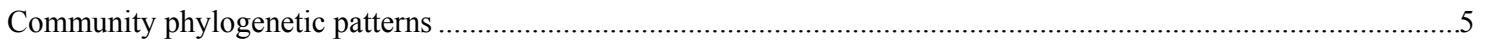

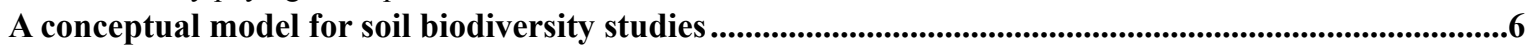

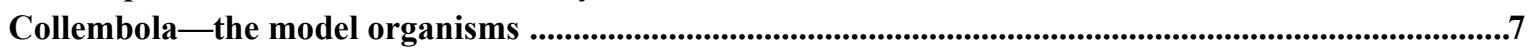

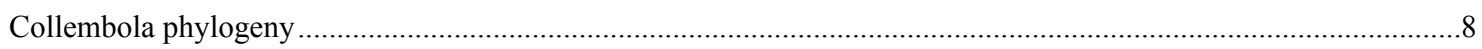

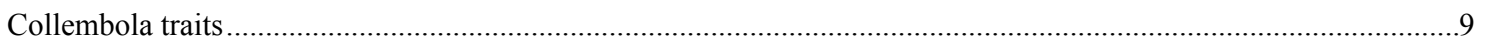

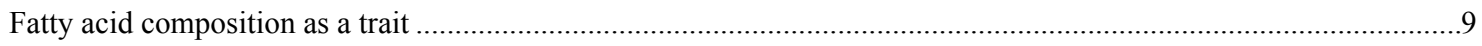

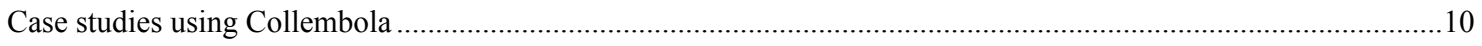

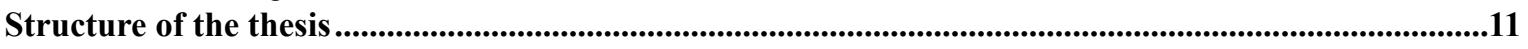

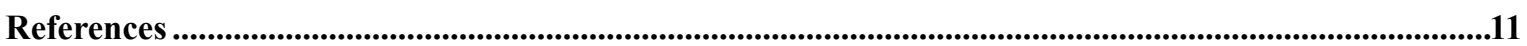

\section{Chapter 2}

Collembola Phylogeny and Trait Evolution ....................................................................17

Abstract ...............................................................................................................................................................17

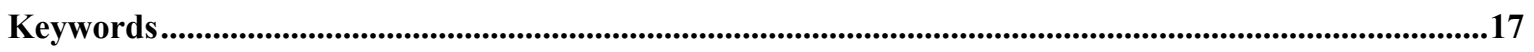

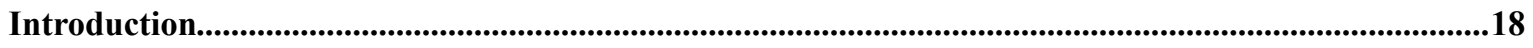

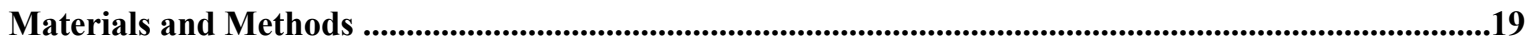

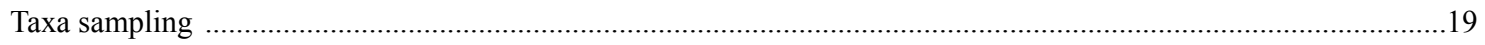

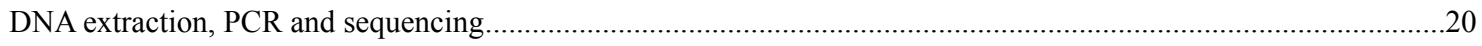

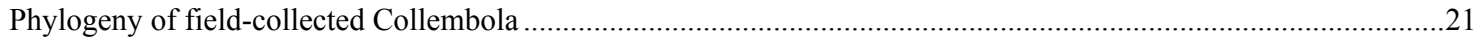

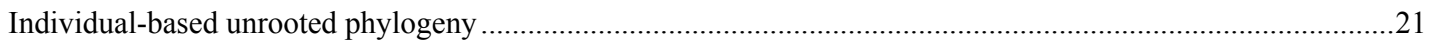

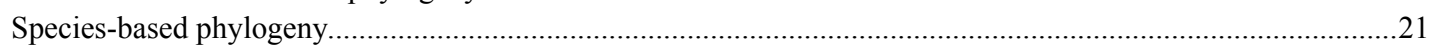

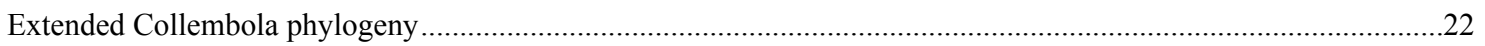

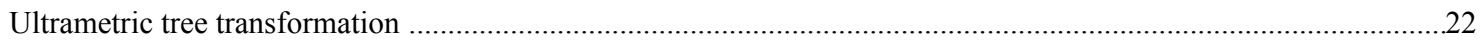

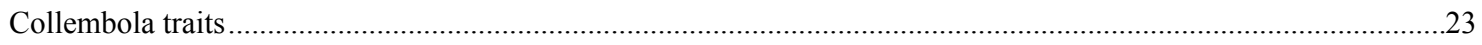

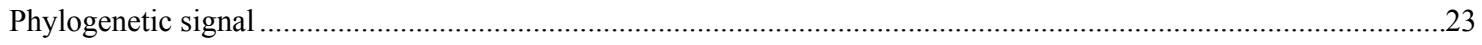

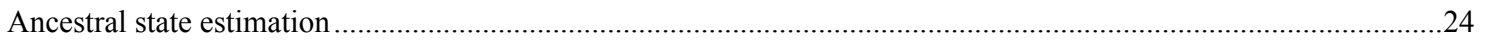

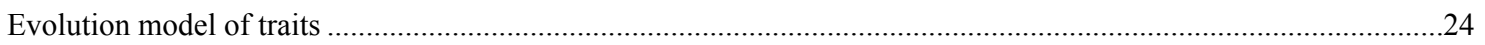

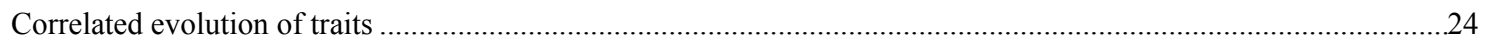

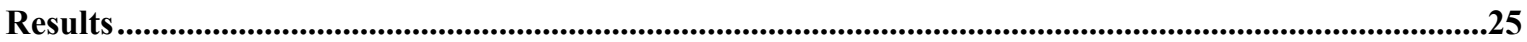

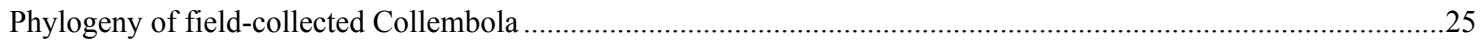

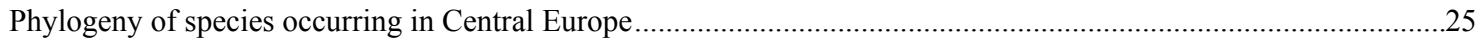

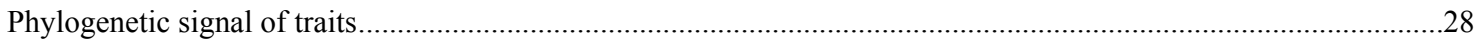

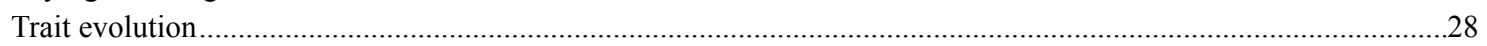

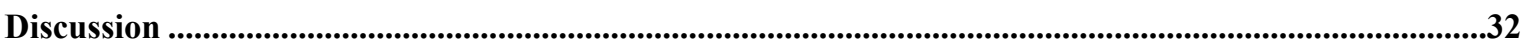

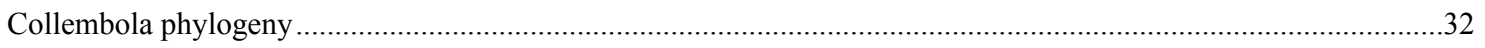

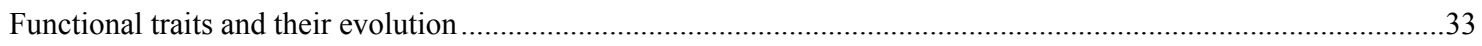

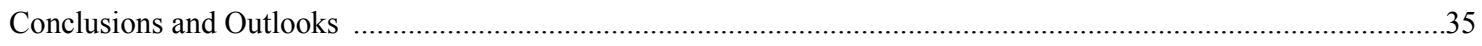

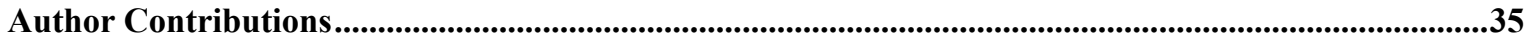

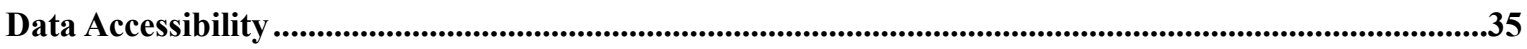

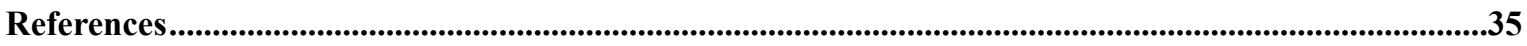

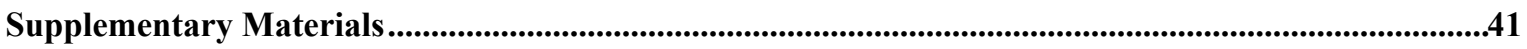




\section{Chapter 3}

Neutral lipid fatty acid composition as trait and constraint in Collembola
evolution

\section{Chapter 4}

\section{Mechanisms of Collembola species coexistence as indicated by phylogeny and}

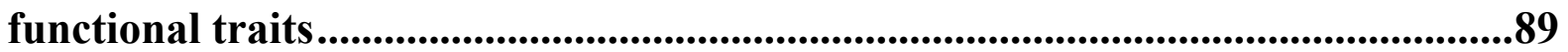

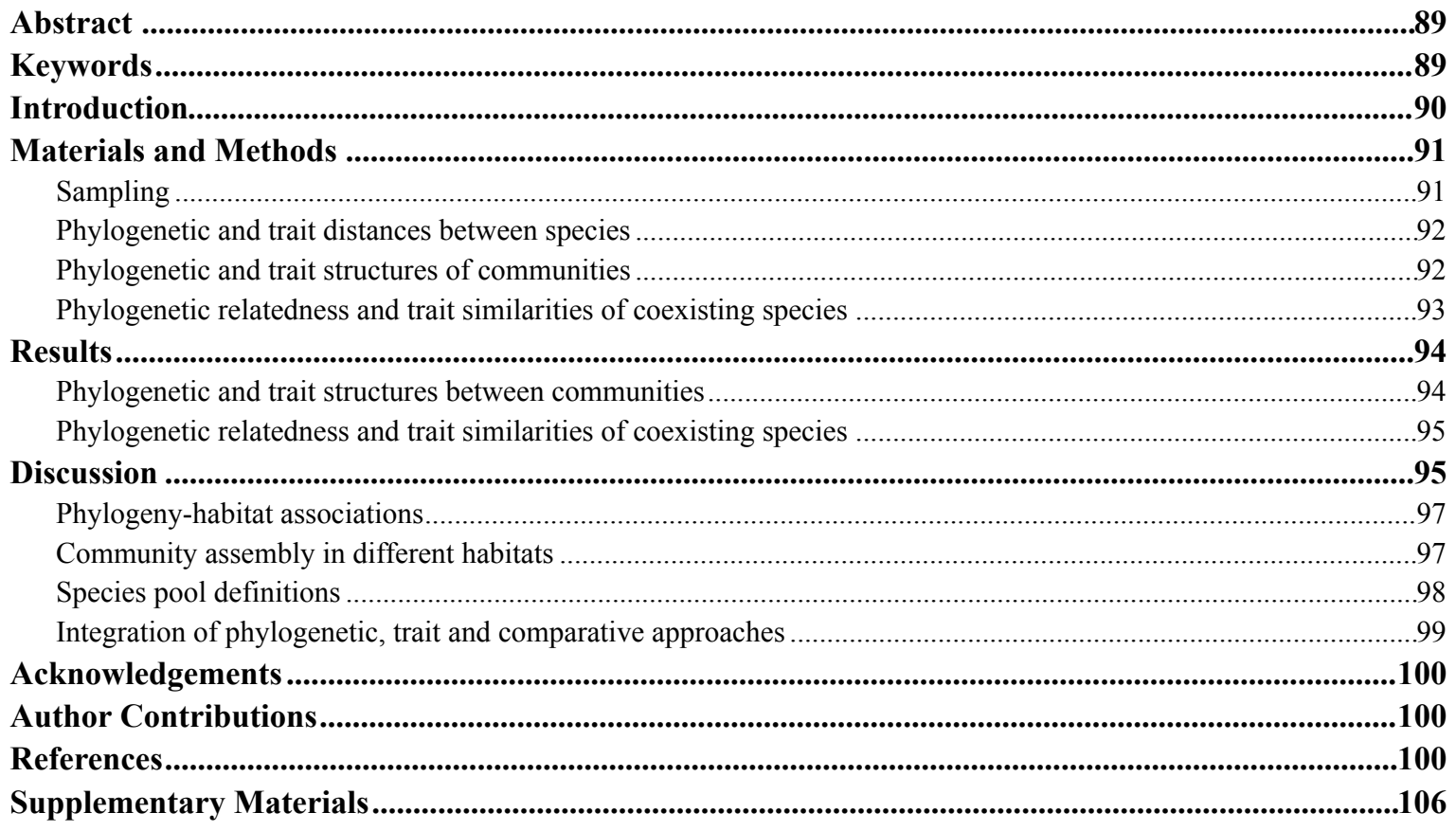




\section{Chapter 5}

\section{Inferring assembly processes of Collembola communities along successional}

trajectories using phylogenetic approaches.......................................................................109

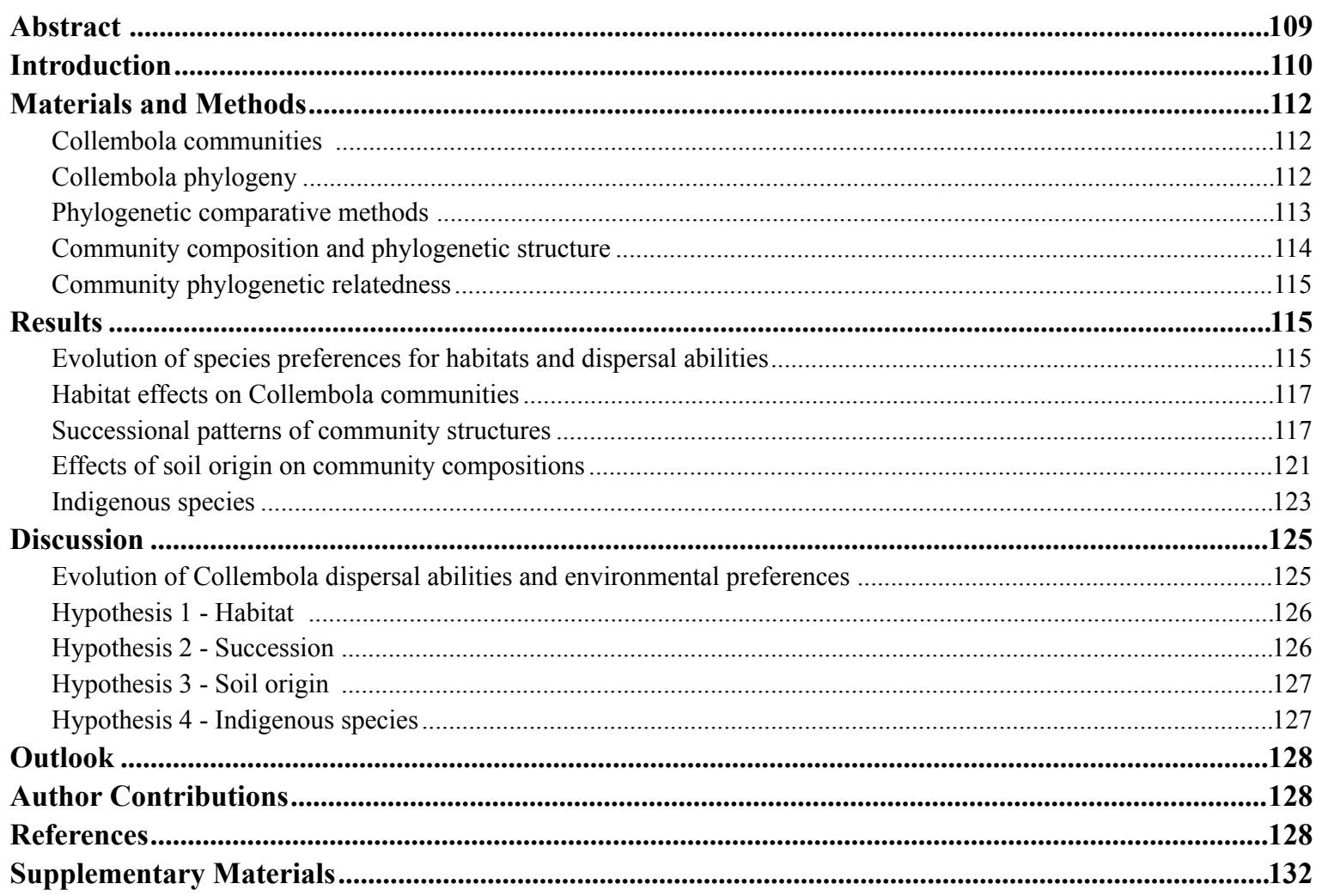

\section{Chapter 6}

\section{General Discussion}

Assembly processes of soil Collembola communities .....................................................................141

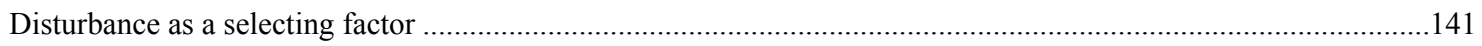

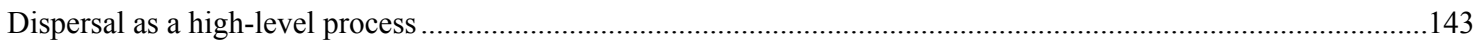

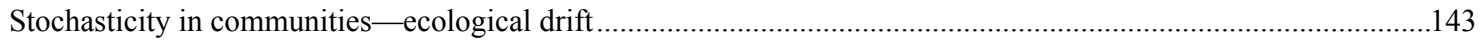

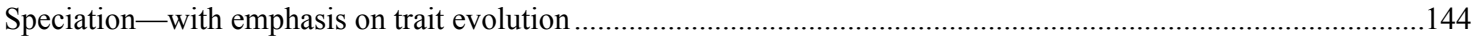

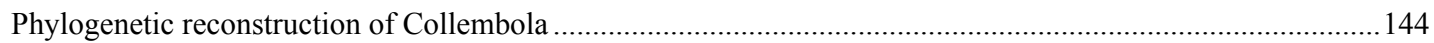

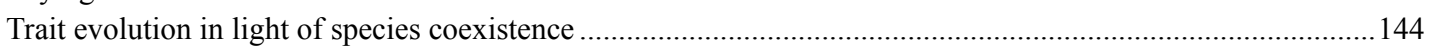

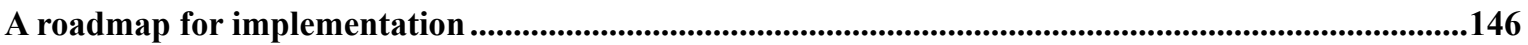

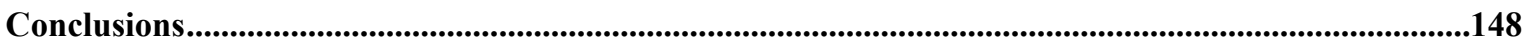

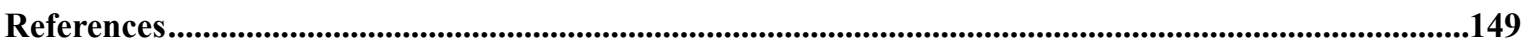

Acknowledgements..............................................................................153

\section{List of publications}

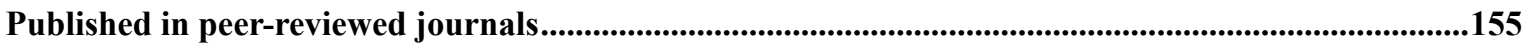

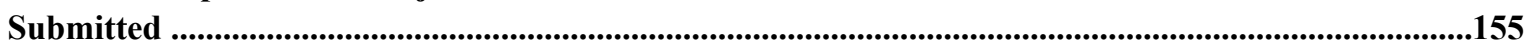

Thesis declarations

Declaration of the author's own contribution to manuscripts with multiple authors ............................157

Plagiarism declaration 


\section{Summary}

Soils are among the most biodiverse systems on earth. The coexistence in soils of a multitude of animal species has long puzzled soil ecologists. How can so many species co-occur, and what are the processes driving and maintaining species coexistence in soil? Using a deductive approach, I propose that (1) there are assembly processes, (2) that work on, or are related to, certain objects, i.e., functional traits, to (3) produce particular patterns. I use a conceptual model combining patterns of evolution of species traits, trait similarity and phylogenetic relatedness between coexisting species, from which to infer assembly processes in soil Collembola (springtail) communities collected from habitats characterized by different disturbance regimes.

In Chapter 2, I reconstruct a Collembola phylogeny and use phylogenetic comparative methods to explore phylogenetic signal, model of evolution and ancestral state for a variety of traits, including body shape, body length, pigmentation, number of ommatidia, vertical stratification and reproductive mode. The results demonstrate that body shape of Collembola evolved quickly early in their diversification but slowed down afterwards. In contrast, evolutionary transitions in pigmentation, number of ommatidia and reproductive mode depended on how deep in the soil that species live. Ancestral Collembola traits were likely slender body, hemiedaphic way of life, sexual reproduction, possession of many ommatidia and bright color, but these traits presumably changed several times during species diversification. The phylogenetic signal detected in these traits forms the basis of further community phylogenetic analyses.

In Chapter 3, I propose the neutral lipid fatty acid composition of Collembola as a functional trait related to both food resources and physiological functions and test phylogenetic signal in fatty acid profiles. Long-chain polyunsaturated fatty acids related to physiological functions demonstrated phylogenetic signal. In contrast, most food resource biomarker fatty acids and the ratios between bacterial, fungal and plant biomarker fatty acids exhibited no phylogenetic signal. These results suggest that Collembola with close phylogenetic affinity experienced similar environments during divergence, while niche partitioning in food resources among closely related species favored species coexistence.

In Chapter 4, I use both community phylogenetic and trait-based approaches to infer the assembly processes of Collembola communities inhabiting arable fields, grasslands and forests. The results indicate that Collembola communities in arable fields were mainly structured by environmental filtering, while niche partitioning dominated in forests. Epedaphic (surface-living) species showed phylogenetic clustering in grasslands and forests, while in forests they also possessed similar traits. Hemiedaphic (sub-surfacedwelling) species were phylogenetically clustered in arable fields and grasslands, but in forests they were phylogenetically overdispersed and had different traits. However, the assembly of euedaphic (soildwelling) communities did not differ from random patterns. Furthermore, different phylogenetic groups of Collembola showed different patterns in the three habitats. These results suggest that Collembola assemblages are driven by different mechanisms in different habitats, with the relative importance of these mechanisms different between soil strata and between phylogenetic lineages. 
Summary

Furthermore, applying community phylogenetic approaches to a manipulative soil block experiment (Auclerc et al. 2009; Soil Biology and Biochemistry 41, 1596-1604) in Chapter 5 shows that Collembola community composition during their succession in forest and meadow soil was determined by the interaction of dispersal and selection processes. Niche partitioning gradually strengthened at later successional stages, offsetting the effects of environmental filtering. As a consequence of dispersal, community composition changed gradually from that resembling the original habitats to that of the new habitats.

In the final chapter I ascribe the above-mentioned patterns to the scenarios presented in the conceptual model and discuss the likely mechanisms, with reference to the four high-level processes, selection, dispersal, drift and speciation, proposed in The Theory of Ecological Communities (Vellend 2016). I provide a roadmap for integrating phylogenetic comparative methods, community phylogenetic analyses and trait-based approaches in studies on the assembly processes of soil communities. Overall, this thesis is the first application of new methods developed in community ecology and evolutionary biology to the study on assembly processes in the soil communities. Future studies using the conceptual model and roadmap proposed in this thesis will advance our understanding of the mechanisms driving and maintaining soil biodiversity from both ecological and evolutionary perspectives. 


\section{Chapter 1}

\section{General Introduction}

\section{The nature of soil biodiversity}

Soils are among the most biodiverse systems on earth and have been viewed as "the poor man's tropical rainforest" (Giller 1996). The coexistence in soils of a multitude of animals has long puzzled soil ecologists, as reflected in the phrase "the enigma of soil animal species diversity" (Anderson 1975). For example, the density of soil mesofauna, animals with a body width between $0.2 \mathrm{~mm}$ and $2 \mathrm{~mm}$, typically ranges between 10,000 and 200,000 individuals $\mathrm{m}^{-2}$, and local species richness in temperate deciduous forests is usually between 60 and 200 species (Petersen and Luxton 1982). How can so many species cooccur, and what are the processes driving and maintaining species coexistence in soil?

\section{Inferring processes from patterns}

For natural communities, assembly processes are hidden. What is observable in nature are the final patterns, the results of various mechanisms working on the members of communities. Using a deductive reasoning approach, I propose that (1) there are assembly processes, (2) that work on, or are related to, certain objects, to (3) produce particular patterns. By designing models of specific assembly processes and deriving the subsequent patterns, it is possible to relate the patterns observed in real communities with those derived from the models, thus inferring the assembly processes.

\section{Community assembly processes}

Theories posit that communities are affected by a number of processes, including niche-related (Chase and Leibold 2003), neutral (Hubbell 2001) and biogeographical processes (Ricklefs 1987). Recently, Vellend $(2010,2016)$ in The Theory of Ecological Communities linked community ecology and evolutionary biology and summarizes a variety of processes into four overarching high-level processes: selection, drift, dispersal and speciation. In this theory, niche-related processes, such as abiotic environmental filtering and interspecific competition, usually considered as major drivers of community composition, are ascribed to selection processes. Studies on soil biotic communities usually fall into this category and focus on e.g., community-environment relationships (Scheu and Schulz 1996, Scheu et al. 2003, Eissfeller et al. 2013). Ecological drift, in line with the neutral theory of biodiversity (Hubbell 2001), emphasizes stochasticity (i.e., rare or unpredictable fluctuations of populations) of local communities. Some studies suggest that the contribution of stochastic drift to soil community assembly is similar to that of deterministic processes (Minor 2011, Caruso et al. 2011, Caruso et al. 2012). Furthermore, dispersal as a high-level process (i.e., movement of individuals between local communities or from source populations) interacts with selection processes and so together influence local community composition. Metacommunity studies of soil invertebrates point to the generality of frequent dispersal of 
individuals between local communities, resulting in mass effects being a dominant metacommunity scenario for soil biota (Ingimarsdóttir et al. 2012, Heiniger et al. 2014). Finally, speciation, as a consequence of selection and dispersal but not usually discussed as part of community ecology, is the only process generating new ecologically relevant phenotypes or traits-the objects on which selection processes can work.

\section{Functional traits-the objects}

Functional traits are properties of species which influence their performance and fitness (Violle et al. 2007, Pey et al. 2014). Ecologically, functional traits regulate the occurrence of species in habitats and the coexistence with other species (McGill et al. 2006, Ackerly and Cornwell 2007, Adler et al. 2013). They are the objects (or targets, media) on which work selection processes, such as environmental filtering and interspecific competition, resulting in certain patterns of traits in local communities. There are two categories of ecological traits, $\alpha$ and $\beta$ niche traits. $\beta$ niche traits determine the environmental tolerance of species, while $\alpha$ niche traits relate to resource exploitation (Ackerly and Cornwell 2007). Similar $\beta$ niche traits but different $\alpha$ niche traits allow species to live under similar environmental conditions but utilize different resources thereby promoting coexistence (Silvertown et al. 2006).

From an evolutionary perspective, traits are the products of the adaptation of species during their evolutionary history. They may exhibit phylogenetic signal (i.e., a statistic pattern where closely related species resemble each other in their trait values), since species inherit similar traits from their common ancestors. Where this occurs, variations in traits between species are predicted by phylogenetic distances. However, environmental constraints in the past may result in more conserved traits than predicted, while other diversifying mechanisms, such as adaptive radiation or competition, may result in trait divergence and therefore in traits being phylogenetically labile. As a consequence, $\beta$ niche traits are usually phylogenetically conserved and exhibit phylogenetic signal, while $\alpha$ niche traits tend to be evolutionarily labile or divergent (Ackerly et al. 2006, Best and Stachowicz 2013). The different evolutionary consequences of $\alpha$ and $\beta$ niche traits allow species coexistence (Silvertown et al. 2006, Ackerly and Cornwell 2007). The phylogenetic signal in functional traits therefore forms a mechanistic link between the evolutionary history of species and the contemporary ecological processes to which they are exposed (Cavender-Bares et al. 2009). Contemporary selection processes, such as environmental filtering and biotic interactions, work on existing traits, resulting in similar and/or different traits among the members of local communities.

Trait-based approaches aim at inferring community assembly processes from the patterns of traits within and between communities. A community with species possessing similar traits is usually inferred to result from environmental filtering, while coexisting species with different traits indicate interspecific competition or niche partitioning (Widenfalk et al. 2015, Widenfalk et al. 2016). Soil ecologists have recently adopted the concept of functional traits, in addition to simple species identity, to investigate 
belowground community structure and its association with the environment (Vandewalle et al. 2010, Pey et al. 2014, Moretti et al. 2017). Commonly used functional traits of soil invertebrates include morphological characters (e.g., body size, pigmentation and eye morphology), life history (or performance) traits (e.g., reproductive mode and fitness), physiological traits (e.g., metabolic rate and desiccation resistance), behavioral traits (e.g., dispersal mode), as well as ecological preferences that interrelate with other traits (Pey et al. 2014, Moretti et al. 2017). Using functional traits has been shown to be more powerful than simply using species identity for predicting the environmental associations of communities in soil (Makkonen et al. 2011, Bokhorst et al. 2012). Therefore, trait-based approaches are increasingly adopted in analyzing soil communities.

However, not all traits relevant to assembly processes can be measured in soil invertebrates (Moretti et al. 2017). Given that functional traits exhibit phylogenetic signal, phylogenetic information about species can be used as a surrogate for functional traits to infer assembly processes (Kembel 2009, Mouquet et al. 2012, Cadotte et al. 2013). Soil invertebrates likely exhibit phylogenetic signal (Pachl et al. 2012, Ponge and Salmon 2013, Potapov et al. 2016, Malcicka et al. 2017), and therefore, in this thesis I adopt the methods of community phylogeny which have been developed among plant ecologists (Webb et al. 2002, Cavender-Bares et al. 2009) and applied these methods to the studies on soil animal communities.

\section{Community phylogenetic patterns}

Community phylogenetic approaches explore the phylogenetic patterns of local communities to investigate the relative contribution of different processes to community assembly (Webb et al. 2002, Cavender-Bares et al. 2009). The essence of the community phylogenetic approach is to compare the observed pattern of phylogenetic distances between species in local communities with that derived from null model communities by randomly drawing species from a pre-defined species pool. If the assumption of phylogenetic conservatism of ecologically relevant traits is accepted, a community composed of phylogenetically closely related species can be inferred to be structured by environmental filtering. The environment may select for species possessing certain conserved traits that cope with specific abiotic conditions. In contrast, low relatedness among coexisting species reflected in each species having different traits points to the dominance of competitive interactions, as species compete for the same resources thereby limiting local coexistence or occupying different niches (Webb et al. 2002, CavenderBares et al. 2009, but see Gerhold et al. 2015).

Furthermore, community phylogenetic approaches are sensitive to spatial and taxonomic scales and the definition of species pool (Cavender-Bares et al. 2006, Swenson et al. 2006, Cavender-Bares et al. 2009). At larger spatial scales, environmental filtering influences local communities more strongly than species interactions, while the species interactions are more influential at finer taxonomic or spatial scales (Cavender-Bares et al. 2006, Swenson et al. 2006, Cavender-Bares et al. 2009). Therefore, using different specific pool definitions at different scales may help to gain deeper insight into the processes working at 
different spatial, temporal and taxonomic levels (Swenson et al. 2006, Emerson and Gillepsie 2008, Lessard et al. 2012).

Community phylogenetic approaches have shown their value for inferring assembly processes in aboveground communities inhabiting various environments characterized by disturbance regimes or harshness. For example, phylogenetic clustering in communities of plants (Webb 2000, Dinnage 2009, Ding et al. 2012), birds (Gianuca et al. 2014), amphibians (Brum et al. 2013) and bees (Pellissier et al. 2013, Sydenham et al. 2016) indicates that intensive disturbance and harshness in environments work predominantly as filtering processes leading to the coexistence of phylogenetically closely related species possessing similar disturbance-adapted traits (Ding et al. 2012, Gianuca et al. 2014). However, community phylogenetic approaches have not yet been commonly applied to soil biota [but see Bässler et al. (2014) and Thorn et al. (2016) for fungi; Li et al. (2014) for nematodes; Hausberger and Korb (2015) and Hausberger and Korb (2016) for termites; Andújar et al. (2015) and Thorn et al. (2016) for beetles], despite the exceptional diversity of soil communities and the varied traits possessed by different species.

\section{A conceptual model for soil biodiversity studies}

In this thesis, I use the conceptual model of Emerson and Gillespie (2008) that considers (1) evolution of species traits, (2) trait similarity and (3) phylogenetic relatedness between coexisting species (Figure 1.1). Patterns of these three measurable elements together produce scenarios of coexisting species that situate somewhere between the four extreme cases:

(a) Conserved traits + similar states + closely related species;

(b) Conserved traits + different states + distantly related species;

(c) Divergent traits + different states + closely related species;

(d) Convergent traits + similar states + distantly related species.

Here, conserved, divergent and convergent indicate how traits have evolved during species diversification, while similar or different indicate the differences in trait states between coexisting species.

Various processes can produce the above-mentioned patterns. In case (a) local species possess similar traits that have evolved in a conserved manner, and the local species are phylogenetically close relatives. This suggests that environmental filtering is the predominant process by selecting species that possess certain traits and therefore resulting in similar traits between coexisting species. The strength of species dispersal is weak, compared to local environmental filtering. This scenario may also suggest sympatric speciation, since species within local communities belong to the same phylogenetic clades and each community is assembled of species from a single clade all of which inherit their traits from the common ancestor. Case (b) provides an example in which the local community is assembled from species of various phylogenetic clades each possessing different traits inherited from their ancestors. This suggests that contemporary competition drives species toward niche partitioning; the local species possess 
different traits. Here, dispersal is intermediate, allowing species from different phylogenetic clades to meet together. In case (c) species possess different traits but assemble from the same phylogenetic clades. Divergent traits resulting from species diversification may suggest historical competition, while different traits among coexisting species indicate niche partitioning. The dispersal ability of the species is likely limited. Finally, in case (d), environmental filtering is likely a predominant process that selects local species with similar traits that evolved convergently, presumably reflecting past competition or adaptation. This scenario also points to substantial dispersal allowing species to colonize new habitats where selection is at work.

\section{(a)}

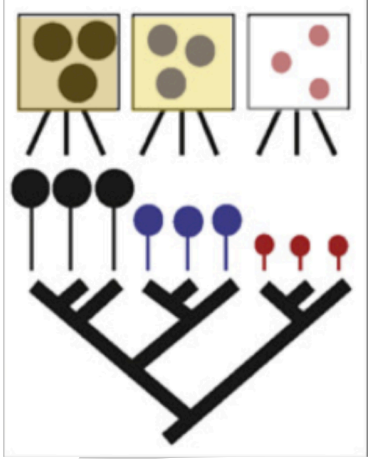

(b)

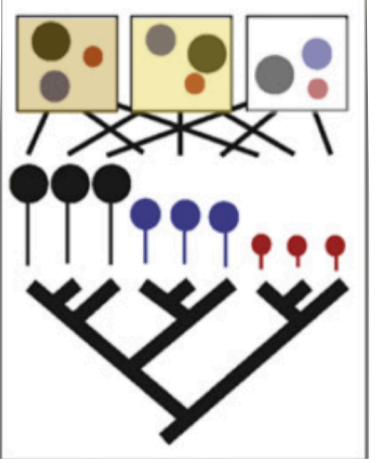

(c)

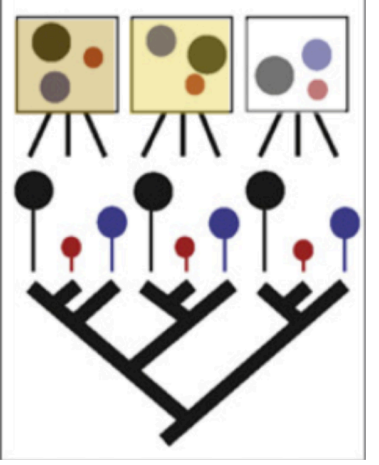

(d)

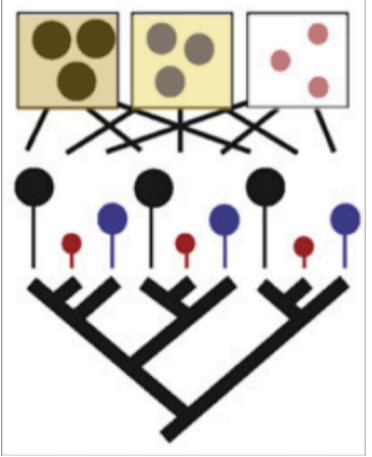

\section{Figure 1.1}

The conceptual model used in this thesis. Possible processes resulting in the four scenarios are (a) sympatric speciation + phylogenetic niche conservatism of trait + environmental filtering, (b) phylogenetic niche conservatism of trait + intermediate dispersal ability + contemporary competition, (c) historical competition + niche partitioning + low dispersal ability and (d) environmental filtering + convergent evolution of trait + substantial dispersal. Figures are modified from Emerson and Gillespie (2008). Squares represent local communities composed of species with different states of a trait (circles with different sizes and colors). Trait states are mapped on the phylogenetic tree, with connecting lines to the communities that indicate phylogenetic belonging of the species in the local communities.

Given that the target objects are known, the patterns are used to infer the processes. This thesis aims at integrating community phylogenetic approaches with phylogenetic comparative and trait-based methods, to study assembly processes in one of the most widespread and abundant soil arthropods, Collembola.

\section{Collembola — the model organisms}

Collembola (springtails) are early-derived Hexapoda characterized among others by a "jumping organ" (furca) at the ventral side of abdomen. The evolution of the furca likely contributed to species diversification (Beutel et al. 2017). The furca presumably evolved to escape predators, but it is reduced or lost in some soil-dwelling species. Another feature that characterizes Collembola is the ventral tube, from which Collembola got their name from Greek colla (glue) and embolus (piston). The ventral tube functions in fluid balance and is presumably important because the habitats of Collembola are usually moist, such as soil, tree canopies, aquatic surfaces, glaciers and caves (Hopkin 1997). 
In soil, Collembola are among the most abundant and diverse mesofauna. Their density may reach 10,000-100,000 individuals $\mathrm{m}^{-2}$, with a typical species richness of 60-80 species in temperate deciduous forests (Petersen and Luxton 1982). They are decomposers, feeding mainly on fungi and decaying organic matter but may also function as omnivores consuming a variety of food resources (Scheu and Falca 2000, Berg et al. 2004, Chahartaghi et al. 2005, Ferlian et al. 2015). They regulate microbial activity and therefore contribute to decomposition processes and nutrient cycling (Petersen and Luxton 1982, Rusek 1998, Schaefer et al. 2009).

Community compositions of Collembola typically vary with habitat characteristics. Different Collembola communities have been reported inhabiting arable fields, grasslands and forests, three types of habitats dominating mosaic landscapes in Central Europe (Ponge et al. 2003, Sousa et al. 2006, Martins da Silva et al. 2012, Heiniger et al. 2014). This thesis aims at investigating soil Collembola communities sampled from habitats characterized by different disturbance regimes by integrating phylogenetic comparative methods, community phylogenetic and trait-based approaches. The challenges in applying such approaches include the lack of knowledge on phylogenetic relationships of Collembola. Also, traits relevant to processes need to be identified and phylogenetic signal of traits needs to be tested.

\section{Collembola phylogeny}

The first fossil record of Collembola is from the Devonian, ca. 400 million years ago (Hirst and Maulik 1926, Whalley and Jarzembowski 1981). Today about 8,600 Collembola species have been described and assigned to four orders, i.e., Poduromorpha, Entomobryomorpha, Neelipleona and Symphypleona (Bellinger et al. 1996-2017; www.collembola.org), with their phylogenetic relationships still in debate. Molecular phylogenetic studies suggest that Symphypleona are sister to the other Collembola taxa, either paraphyletic (D'Haese 2002, Luan et al. 2005) or monophyletic (Xiong et al. 2008, Schneider et al. 2011, Yu et al. 2016) and that Entomobryomorpha are paraphyletic due to the position of Tomoceridae (Schneider et al. 2011). Furthermore, the phylogenetic position of Neelipleona is not resolved, although they are likely more closely related to Entomobryomorpha than to Symphypleona (Deharveng 2004).

However, previous phylogenetic studies of Collembola either were based on few genetic markers (D'Haese 2002) or few taxa (Xiong et al. 2008), or focused on single taxonomic groups (Schneider et al. 2011, Yu et al. 2016). Different marker selection, unbalanced taxon sampling and choice of outgroup taxa may lead to inconsistency in phylogenetic inference (Heath et al. 2008, Rosenfeld et al. 2012). Since phylogenetic relationships of Collembola species are still not resolved, in the thesis I first construct phylogenetic trees for locally occurring Collembola species sampled from arable fields, grasslands and forests, and then apply the trees in the subsequent studies on trait evolution and community phylogeny. 


\section{Collembola traits}

Collembola are among the few soil taxa for which trait databases are available (Vandewalle et al. 2010, Pey et al. 2014, Matty P. Berg, unpublished data). Collembola traits, such as reproductive modes and morphological characters, are associated with the environment (Makkonen et al. 2011, Bokhorst et al. 2012, Salmon and Ponge 2012, Salmon et al. 2014, Widenfalk et al. 2015). Spherical body shape, large body size, dark pigmentation and sexual reproduction are characteristic of species occurring in open habitats and at the soil surface, whereas small body size, lack of eye spots, pale color and asexual reproduction are typical traits of species inhabiting forests and living in soil (Salmon et al. 2014). That particular traits are associated with habitats suggest that community assembly processes are driven by selection processes (Vellend 2016), e.g. environmental filtering or interspecific competition (Widenfalk et al. 2015, Widenfalk et al. 2016).

In this thesis, I use body shape, body length, pigmentation, number of ommatidia, vertical stratification and reproductive mode as traits of Collembola. Phylogenetic signal of these traits is analyzed using comparative methods. To link traits that presumably underlie contemporary assembly processes to evolutionary processes, I estimate ancestral character states of the traits and compare models of trait evolution. Furthermore, I propose neutral lipid fatty acid composition as a trait that can reflect both food resources and physiological attributes of different species.

\section{Fatty acid composition as a trait}

Neutral lipid fatty acids (NLFAs) in fat deposits of consumers are commonly used to identify diets of soil animals (Ruess and Chamberlain 2010, Ferlian et al. 2015). Specific NLFAs directly incorporated from food resources without modification in consumers carry signatures of food resources ('dietary routing'). Thus, these NLFAs are used as biomarkers to identify the origin of food. As omnivores in soil, Collembola consume a wide range of food resources including detritus, roots and root exudates, bacteria, fungi and algae (Hopkin 1997), which are difficult to measure directly in the field. By examining biomarker fractions of NLFAs in Collembola, the dietary routes can be identified (Chamberlain et al. 2005, Ferlian et al. 2015). Proportions of different biomarker NLFAs may be considered as a trophic trait , i.e., an $\alpha$ niche trait that reflects food resources.

Other NLFAs are synthesized or modified by consumers from precursors and then further integrated into other compounds. For example, long-chain polyunsaturated fatty acids are essential for biosynthesis of prostaglandins and eicosanoids, which are associated with reproduction, immune response and temperature regulation (Chamberlain et al. 2004, Chamberlain and Black 2005, Haubert et al. 2008, Ruess and Chamberlain 2010). Proportions of these fatty acids may thus represent physiological attributes, i.e., $\beta$ niche traits that reflect environmental requirements of species. 
In this thesis, I analyze NLFA profiles of field-sampled Collembola. A further compilation of literature-reported NLFA profiles in other Collembola species allows phylogenetic signal to be measured in a broader context.

\section{Case studies using Collembola}

To conduct community phylogenetic analyses for studying assembly processes of Collembola communities, three datasets are needed: a phylogenetic tree, a trait matrix and a species-site (community) matrix. Assembly processes are inferred from the patterns of phylogenetic relatedness and trait similarity of the coexisting species in local communities (an $\alpha$-diversity approach; Webb et al. 2002). The observed patterns in communities are compared with those generated by null models, where species are randomly selected from a pre-defined species pool. The species pool can be defined in various ways, for example, at different taxonomic levels or for species with certain traits e.g., vertical stratification in soil profile. In this thesis I focus on the comparison of species coexistence patterns between Collembola communities inhabiting arable fields, grasslands and forests. Since these habitats are characterized by distinct disturbance regimes and vegetation, community assembly processes in soil are likely different between habitats. In a disturbed habitat, Collembola assemblages are likely to be driven by environmental filtering, resulting in similar traits and phylogenetically related species coexisting locally (Widenfalk et al. 2015). In contrast, different traits of distant relatives coexisting in a stable environment suggest that communities are predominantly influenced by niche partitioning or interspecific competition (Widenfalk et al. 2016).

I also use a metacommunity phylogenetic approach (phylogenetic $\beta$-diversity; Graham and Fine 2008). In distinction to traditional $\beta$-diversity studies where species are treated independently, phylogenetic $\beta$ diversity considers similarity in traits and phylogeny between species when exploring dissimilarities between local communities thus allowing regional or evolutionary processes to be connected to local processes, such as environmental filtering and interspecific competition (Graham and Fine 2008). Accounting for non-independence between coexisting species in the traits and phylogeny can help to investigate community-environmental associations from an evolutionary perspective (Pillar and Duarte 2010, Duarte 2011, Duarte et al. 2016).

Furthermore, inferring processes from patterns may benefit from experimental manipulations, compensating for the insufficiency of process inference in pure observational studies (Weber and Agrawal 2012). Controlling for factors that potentially influence community assembly is a necessity if processes are to be explicitly tested. In this respect, soil animal communities are more easy to manipulate than those of higher plants or vertebrates, for example, by defaunation (removing animals from soil) followed by the translocation of soil blocks (Ponge et al. 2008, Auclerc et al. 2009, Heiniger et al. 2015). Taking the data of Collembola communities from the experiment conducted by Auclerc et al. (2009) that aimed at ascribing species to different groups of dispersal ability and habitat preferences, I reanalyze 
species compositions using (meta)community phylogenetic approaches, to understand assembly processes that result from dispersal, are determined by soil properties, and differ at different successional stages.

\section{Structure of the thesis}

The aim of this thesis is to investigate assembly processes of soil Collembola communities in different types of habitats using phylogenetic comparative methods, community phylogenetic approaches and traitbased analyses. The general hypothesis tested here is that species in disturbed habitats are determined by environmental filtering that results in phylogenetic clustering and similar traits, while in relatively stable habitats interspecific competition or niche partitioning is predominant that leads to phylogenetic overdispersion with species possessing different traits, given that traits exhibit phylogenetic signal.

In Chapter 2, I construct Collembola phylogeny and use phylogenetic comparative methods to explore phylogenetic signal, model of evolution and ancestral state for a variety of traits, including body shape, body length, pigmentation, number of ommatidia, vertical stratification and reproductive mode. In Chapter 3, fatty acid composition is considered as a trait of Collembola and its phylogenetic signal is measured. Chapter 4 aims at inferring community assembly processes of Collembola inhabiting arable fields, grasslands and forests using community phylogenetic approaches. Further in Chapter 5, I test for phylogenetic signal in habitat/soil preferences and dispersal abilities of Collembola species reported in Auclerc et al. (2009), and with a specific focus on assembly processes I explore the phylogenetic patterns in communities derived from the experimental designs. In Chapter 6, I ascribe the patterns of Collembola communities found in this thesis to the scenarios in the conceptual model (Figure 1.1), discuss the likely processes referring to the four high-level processes in The Theory of Ecological Communities (Vellend 2010, 2016) including selection, dispersal, drift and speciation, and propose a roadmap for soil ecologists to integrate phylogenetic comparative methods, community phylogenetic analyses and traitbased approaches in studies on the assembly processes of soil communities.

\section{References}

Ackerly, D.D. \& Cornwell, W.K. (2007) A trait-based approach to community assembly: Partitioning of species trait values into within- and among-community components. Ecology Letters, 10, 135-145.

Ackerly, D.D., Schwilk, D.W. \& Webb, C.O. (2006) Niche evolution and adaptive radiation: testing the order of trait divergence. Ecology, 87, S50-61.

Adler, P.B., Fajardo, A., Kleinhesselink, A.R. \& Kraft, N.J.B. (2013) Trait-based tests of coexistence mechanisms. Ecology Letters, 16, 1294-1306.

Anderson, J.M. (1975) The enigma of soil animal species diversity. Progress in Soil Zoology: Proceedings of the 5th International Colloquium on Soil Zoology (ed J. Vaněk), pp. 51-58. Springer Netherlands, Dordrecht.

Andújar, C., Arribas, P., Ruzicka, F., Platt, A.C., Timmermans, M.J.T.N. \& Vogler, A.P. (2015) Phylogenetic community ecology of soil biodiversity using mitochondrial metagenomics. Molecular Ecology, 24, 3603-3617. 
Auclerc, A., Ponge, J.-F., Barot, S. \& Dubs, F. (2009) Experimental assessment of habitat preference and dispersal ability of soil springtails. Soil Biology and Biochemistry, 41, 1596-1604.

Bässler, C., Ernst, R., Cadotte, M., Heibl, C. \& Müller, J. (2014) Near-to-nature logging influences fungal community assembly processes in a temperate forest (ed J Barlow). Journal of Applied Ecology, 51, 939-948.

Bellinger, P.F., Christiansen, K.A. \& Janssens, F. Checklist of the Collembola of the world (www.collembola.org).

Berg, M.P., Stoffer, M. \& van den Heuvel, H.H. (2004) Feeding guilds in Collembola based on digestive enzymes. Pedobiologia, 48, 589-601.

Best, R.J. \& Stachowicz, J.J. (2013) Phylogeny as a proxy for ecology in seagrass amphipods: which traits are most conserved? PLOS ONE, 8, e57550.

Beutel, R.G., Yavorskaya, M., Mashimo, Y., Fukui, M. \& Meusemann, K. (2017) The phylogeny of Hexapoda (Arthropoda) and the evolution of megadiversity. Proceedings of the Arthropodan Embryological Society of Japan, 51, 115.

Bokhorst, S., Phoenix, G.K., Bjerke, J.W., Callaghan, T. V., Huyer-Brugman, F. \& Berg, M.P. (2012) Extreme winter warming events more negatively impact small rather than large soil fauna: shift in community composition explained by traits not taxa. Global Change Biology, 18, 1152-1162.

Brum, F.T., Gonçalves, L.O., Cappelatti, L., Carlucci, M.B., Debastiani, V.J., Salengue, E.V., dos Santos Seger, G.D., Both, C., Bernardo-Silva, J.S., Loyola, R.D. \& da Silva Duarte, L. (2013) Land use explains the distribution of threatened New World amphibians better than climate. PLoS ONE, 8, 4-11.

Cadotte, M., Albert, C.H. \& Walker, S.C. (2013) The ecology of differences: assessing community assembly with trait and evolutionary distances. Ecology Letters, 16, 1234-1244.

Caruso, T., Chan, Y., Lacap, D.C., Lau, M.C., McKay, C.P. \& Pointing, S.B. (2011) Stochastic and deterministic processes interact in the assembly of desert microbial communities on a global scale. The ISME Journal, 5, 14061413.

Caruso, T., Taormina, M. \& Migliorini, M. (2012) Relative role of deterministic and stochastic determinants of soil animal community: a spatially explicit analysis of oribatid mites. The Journal of Animal Ecology, 81, $214-221$.

Cavender-Bares, J., Keen, A. \& Miles, B. (2006) Phylogenetic structure of Floridian plant communities depends on taxonomic and spatial scale. Ecology, 87, S109-22.

Cavender-Bares, J., Kozak, K.H., Fine, P. V \& Kembel, S.W. (2009) The merging of community ecology and phylogenetic biology. Ecology Letters, 12, 693-715.

Chahartaghi, M., Langel, R., Scheu, S. \& Ruess, L. (2005) Feeding guilds in Collembola based on nitrogen stable isotope ratios. Soil Biology and Biochemistry, 37, 1718-1725.

Chamberlain, P.M. \& Black, H.I.J. (2005) Fatty acid compositions of Collembola: unusually high proportions of C20 polyunsaturated fatty acids in a terrestrial invertebrate. Comparative Biochemistry and Physiology Part B: Biochemistry and Molecular Biology, 140, 299-307.

Chamberlain, P.M., Bull, I.D., Black, H.I., Ineson, P. \& Evershed, R.P. (2004) Lipid content and carbon assimilation in Collembola: implications for the use of compound-specific carbon isotope analysis in animal dietary studies. Oecologia, 139, 325-335.

Chamberlain, P.M., Bull, I.D., Black, H.I.J., Ineson, P. \& Evershed, R.P. (2005) Fatty acid composition and change in Collembola fed differing diets: identification of trophic biomarkers. Soil Biology and Biochemistry, 37, 1608-1624.

Chase, J.M. \& Leibold, M.A. (2003) Ecological Niches: Linking Classical and Contemporary Approaches. University of Chicago Press, Chicago, IL.

D'Haese, C.A. (2002) Were the first springtails semi-aquatic? A phylogenetic approach by means of 28S rDNA and optimization alignment. Proceedings of the Royal Society B, 269, 1143-1151.

Deharveng, L. (2004) Recent advances in Collembola systematics. Pedobiologia, 48, 415-433. 
Ding, Y., Zang, R., Letcher, S.G., Liu, S. \& He, F. (2012) Disturbance regime changes the trait distribution, phylogenetic structure and community assembly of tropical rain forests. Oikos, 121, 1263-1270.

Dinnage, R. (2009) Disturbance alters the phylogenetic composition and structure of plant communities in an old field system. PLOS ONE, 4, e7071.

Duarte, L.D.S. (2011) Phylogenetic habitat filtering influences forest nucleation in grasslands. Oikos, 120, $208-215$.

Duarte, L.D.S., Debastiani, V.J., Freitas, A.V.L. \& Pillar, V.D. (2016) Dissecting phylogenetic fuzzy weighting: theory and application in metacommunity phylogenetics. Methods in Ecology and Evolution, 7, 937-946.

Eissfeller, V., Langenbruch, C., Jacob, A., Maraun, M. \& Scheu, S. (2013) Tree identity surpasses tree diversity in affecting the community structure of oribatid mites (Oribatida) of deciduous temperate forests. Soil Biology and Biochemistry, 63, 154-162.

Emerson, B.C. \& Gillespie, R.G. (2008) Phylogenetic analysis of community assembly and structure over space and time. Trends in Ecology \& Evolution, 23, 619-630.

Ferlian, O., Klarner, B., Langeneckert, A.E. \& Scheu, S. (2015) Trophic niche differentiation and utilisation of food resources in collembolans based on complementary analyses of fatty acids and stable isotopes. Soil Biology and Biochemistry, 82, 28-35.

Gerhold, P., Cahill, J.F., Winter, M., Bartish, I. V \& Prinzing, A. (2015) Phylogenetic patterns are not proxies of community assembly mechanisms (they are far better). Functional Ecology, 29, 600-614.

Gianuca, A.T., Dias, R.A., Debastiani, V.J. \& Duarte, L.D.S. (2014) Habitat filtering influences the phylogenetic structure of avian communities across a coastal gradient in southern Brazil. Austral Ecology, 39, 29-38.

Giller, P.S. (1996) The diversity of soil communities, the 'poor man's tropical rainforest'. Biodiversity and Conservation, 5, 135-168.

Graham, C.H. \& Fine, P. V. (2008) Phylogenetic beta diversity: linking ecological and evolutionary processes across space in time. Ecology Letters, 11, 1265-1277.

Haubert, D., Häggblom, M.M., Scheu, S. \& Ruess, L. (2008) Effects of temperature and life stage on the fatty acid composition of Collembola. European Journal of Soil Biology, 44, 213-219.

Hausberger, B. \& Korb, J. (2015) A phylogenetic community approach for studying termite communities in a West African savannah. Biology Letters, 11, 20150625.

Hausberger, B. \& Korb, J. (2016) The impact of anthropogenic disturbance on assembly patterns of termite communities. Biotropica, 48, 356-364.

Heath, T.A., Hedtke, S.M. \& Hillis, D.M. (2008) Taxon sampling and the accuracy of phylogenetic analyses. Journal of Systematics and Evolution, 46, 239-257.

Heiniger, C., Barot, S., Ponge, J.-F., Salmon, S., Botton-Divet, L., Carmignac, D. \& Dubs, F. (2014) Effect of habitat spatiotemporal structure on collembolan diversity. Pedobiologia, 57, 103-117.

Heiniger, C., Barot, S., Ponge, J.-F., Salmon, S., Meriguet, J., Carmignac, D., Suillerot, M. \& Dubs, F. (2015) Collembolan preferences for soil and microclimate in forest and pasture communities. Soil Biology and Biochemistry, 86, 181-192.

Hirst, S. \& Maulik, S. (1926) On some arthropod remains from the Rhynie Chert (old red sandstone). Geological Magazine, 63, 69-71.

Hopkin, S.P. (1997) Biology of the Springtails (Insecta: Collembola). Oxford University Press, Oxford.

Hubbell, S.P. (2001) The Unified Neutral Theory of Biodiversity and Biogeography. Princeton University Press, Princeton and Oxford.

Ingimarsdóttir, M., Caruso, T., Ripa, J., Magnusdottir, O.B., Migliorini, M. \& Hedlund, K. (2012) Primary assembly of soil communities: disentangling the effect of dispersal and local environment. Oecologia, 170, 745-754. 
Kembel, S.W. (2009) Disentangling niche and neutral influences on community assembly: assessing the performance of community phylogenetic structure tests. Ecology letters, 12, 949-60.

Lessard, J.-P., Belmaker, J., Myers, J.A., Chase, J.M. \& Rahbek, C. (2012) Inferring local ecological processes amid species pool influences. Trends in Ecology \& Evolution, 27, 600-607.

Li, J., Li, S., Chen, Y., Jia, P., Hua, Z., Wang, S., Song, Y., Liao, B. \& Shu, W. (2014) Phylogenetic structures of soil nematode communities along a successional gradient in an unreclaimed copper mine tailings site. Soil Biology and Biochemistry, 77, 179-186.

Luan, Y., Mallatt, J.M., Xie, R.D., Yang, Y.M. \& Yin, W. (2005) The phylogenetic positions of three basal-hexapod groups (Protura, Diplura, and Collembola) based on ribosomal RNA gene sequences. Molecular Biology and Evolution, 22, 1579-1592.

Makkonen, M., Berg, M.P., van Hal, J.R., Callaghan, T. V., Press, M.C. \& Aerts, R. (2011) Traits explain the responses of a sub-arctic Collembola community to climate manipulation. Soil Biology and Biochemistry, 43, 377-384.

Malcicka, M., Berg, M.P. \& Ellers, J. (2017) Ecomorphological adaptations in Collembola in relation to feeding strategies and microhabitat. European Journal of Soil Biology, 78, 82-91.

Martins da Silva, P., Berg, M.P., Serrano, A.R.M., Dubs, F. \& Sousa, J.P. (2012) Environmental factors at different spatial scales governing soil fauna community patterns in fragmented forests. Landscape Ecology, 27, 1337-1349.

McGill, B.J., Enquist, B.J., Weiher, E. \& Westoby, M. (2006) Rebuilding community ecology from functional traits. Trends in Ecology \& Evolution, 21, 178-185.

Minor, M.A. (2011) Spatial patterns and local diversity in soil oribatid mites (Acari: Oribatida) in three pine plantation forests. European Journal of Soil Biology, 47, 122-128.

Moretti, M., Dias, A.T.C., de Bello, F., Altermatt, F., Chown, S.L., Azcarate, F.M., Bell, J.R., Fournier, B., Hedde, M., Hortal, J., Ibanez, S., Öckinger, E., Sousa, J.P., Ellers, J. \& Berg, M.P. (2017) Handbook of protocols for standardized measurement of terrestrial invertebrate functional traits. Functional Ecology, 31, 558-567.

Mouquet, N., Devictor, V., Meynard, C.N., Munoz, F., Bersier, L.F., Chave, J., Couteron, P., Dalecky, A., Fontaine, C., Gravel, D., Hardy, O.J., Jabot, F., Lavergne, S., Leibold, M.A., Mouillot, D., Munkemuller, T., Pavoine, S., Prinzing, A., Rodrigues, A.S., Rohr, R.P., Thebault, E. \& Thuiller, W. (2012) Ecophylogenetics: advances and perspectives. Biological Reviews, 87, 769-785.

Pachl, P., Domes, K., Schulz, G., Norton, R., Scheu, S., Schaefer, I. \& Maraun, M. (2012) Convergent evolution of defense mechanisms in oribatid mites (Acari, Oribatida) shows no "ghosts of predation past." Molecular Phylogenetics and Evolution, 65, 412-420.

Pellissier, L., Pradervand, J.-N., Williams, P.H., Litsios, G., Cherix, D. \& Guisan, A. (2013) Phylogenetic relatedness and proboscis length contribute to structuring bumblebee communities in the extremes of abiotic and biotic gradients (ed A Baselga). Global Ecology and Biogeography, 22, 577-585.

Petersen, H. \& Luxton, M. (1982) A comparative analysis of soil fauna populations and their role in decomposition processes. Oikos, 39, 288-388.

Pey, B., Nahmani, J., Auclerc, A., Capowiez, Y., Cluzeau, D., Cortet, J., Decaëns, T., Deharveng, L., Dubs, F., Joimel, S., Briard, C., Grumiaux, F., Laporte, M.-A.A., Pasquet, A., Pelosi, C., Pernin, C., Ponge, J.-F., Salmon, S., Santorufo, L. \& Hedde, M. (2014) Current use of and future needs for soil invertebrate functional traits in community ecology. Basic and Applied Ecology, 15, 194-206.

Pillar, V.D. \& Duarte, L.D.S. (2010) A framework for metacommunity analysis of phylogenetic structure. Ecology Letters, 13, 587-596.

Ponge, J.-F., Gillet, S., Dubs, F., Fedoroff, E., Haese, L., Sousa, J.P. \& Lavelle, P. (2003) Collembolan communities as bioindicators of land use intensification. Soil Biology and Biochemistry, 35, 813-826. 
Ponge, J.-F. \& Salmon, S. (2013) Spatial and taxonomic correlates of species and species trait assemblages in soil invertebrate communities. Pedobiologia, 56, 129-136.

Ponge, J.-F., Tully, T. \& Gins, A. (2008) Short-term responses of two collembolan communities after abrupt environmental perturbation: A field experimental approach. Pedobiologia, 52, 19-28.

Potapov, A.A., Semenina, E.E., Korotkevich, A.Y., Kuznetsova, N.A. \& Tiunov, A. V. (2016) Connecting taxonomy and ecology: Trophic niches of collembolans as related to taxonomic identity and life forms. Soil Biology and Biochemistry, 101, 20-31.

Ricklefs, R.E. (1987) Community diversity: Relative roles of local and regional processes. Science, 235, 167-171.

Rosenfeld, J.A., Payne, A. \& DeSalle, R. (2012) Random roots and lineage sorting. Molecular Phylogenetics and Evolution, 64, 12-20.

Ruess, L. \& Chamberlain, P.M. (2010) The fat that matters: Soil food web analysis using fatty acids and their carbon stable isotope signature. Soil Biology and Biochemistry, 42, 1898-1910.

Rusek, J. (1998) Biodiversity of Collembola and their functional role in the ecosystem. Biodiversity and Conservation, 7 , 1207-1219.

Salmon, S. \& Ponge, J.-F. (2012) Species traits and habitats in springtail communities: A regional scale study. Pedobiologia, 55, 295-301.

Salmon, S., Ponge, J.-F., Gachet, S., Deharveng, L., Lefebvre, N. \& Delabrosse, F. (2014) Linking species, traits and habitat characteristics of Collembola at European scale. Soil Biology and Biochemistry, 75, 73-85.

Schaefer, M., Migge-Kleian, S. \& Scheu, S. (2009) The role of soil fauna for decomposition of plant residues. Functioning and Management of European Beech Ecosystems (eds R. Brumme \& P.K. Khanna), pp. 207-230. Springer Berlin Heidelberg, Berlin, Heidelberg.

Scheu, S., Albers, D., Alphei, J., Buryn, R., Klages, U., Migge, S., Platner, C. \& Salamon, J.-A. (2003) The soil fauna community in pure and mixed stands of beech and spruce of different age: trophic structure and structuring forces. Oikos, 101, 225-238.

Scheu, S. \& Falca, M. (2000) The soil food web of two beech forests (Fagus sylvatica) of contrasting humus type: stable isotope analysis of a macro- and a mesofauna-dominated community. Oecologia, 123, 285-296.

Scheu, S. \& Schulz, E. (1996) Secondary succession, soil formation and development of a diverse community of oribatids and saprophagous soil macro-invertebrates. Biodiversity and Conservation, 5, 235-250.

Schneider, C., Cruaud, C. \& D’Haese, C.A. (2011) Unexpected diversity in Neelipleona revealed by molecular phylogeny approach (Hexapoda, Collembola). Soil Organisms, 83, 383-398.

Silvertown, J., McConway, K., Gowing, D., Dodd, M., Fay, M.F., Joseph, J.A. \& Dolphin, K. (2006) Absence of phylogenetic signal in the niche structure of meadow plant communities. Proceedings of the Royal Society B, 273, 3944.

Sousa, J.P., Bolger, T., da Gama, M.M., Lukkari, T., Ponge, J.-F., Simón, C., Traser, G., Vanbergen, A.J., Brennan, A., Dubs, F., Ivitis, E., Keating, A., Stofer, S. \& Watt, A.D. (2006) Changes in Collembola richness and diversity along a gradient of land-use intensity: A pan European study. Pedobiologia, 50, 147-156.

Swenson, N.G., Enquist, B.J., Pither, J., Thompson, J. \& Zimmerman, J.K. (2006) The problem and promise of scale dependency in community phylogenetics. Ecology, 87, 2418-2424.

Sydenham, M.A.K., Moe, S.R., Stanescu-Yadav, D.N., Totland, Ø. \& Eldegard, K. (2016) The effects of habitat management on the species, phylogenetic and functional diversity of bees are modified by the environmental context. Ecology and Evolution, 6, 961-973.

Thorn, S., Bässler, C., Bernhardt-Römermann, M., Cadotte, M., Heibl, C., Schäfer, H., Seibold, S. \& Müller, J. (2016) Changes in the dominant assembly mechanism drives species loss caused by declining resources. Ecology Letters, 19, 163-170. 
Vandewalle, M., de Bello, F., Berg, M.P., Bolger, T., Dolédec, S., Dubs, F., Feld, C.K., Harrington, R., Harrison, P.A., Lavorel, S., Silva, P.M., Moretti, M., Niemelä, J., Santos, P., Sattler, T., Sousa, J.P., Sykes, M.T., Vanbergen, A.J. \& Woodcock, B.A. (2010) Functional traits as indicators of biodiversity response to land use changes across ecosystems and organisms. Biodiversity and Conservation, 19, 2921-2947.

Vellend, M. (2010) Conceptual synthesis in community ecology. The Quarterly review of biology, 85, 183-206.

Vellend, M. (2016) The Theory of Ecological Communities. Princeton University Press, Princeton.

Violle, C., Navas, M.L., Vile, D., Kazakou, E., Fortunel, C., Hummel, I. \& Garnier, E. (2007) Let the concept of trait be functional! Oikos, 116, 882-892.

Webb, C.O. (2000) Exploring the Phylogenetic Structure of Ecological Communities: An Example for Rain Forest Trees. The American Naturalist, 156, 145-155.

Webb, C.O., Ackerly, D.D., McPeek, M.A. \& Donoghue, M.J. (2002) Phylogenies and community ecology. Annual Review of Ecology and Systematics, 33, 475-505.

Weber, M.G. \& Agrawal, A. a. (2012) Phylogeny, ecology, and the coupling of comparative and experimental approaches. Trends in Ecology \& Evolution, 27, 394-403.

Whalley, P. \& Jarzembowski, E.A. (1981) A new assessment of Rhyniella, the earliest known insect, from the Devonian of Rhynie, Scotland. Nature, 291, 317.

Widenfalk, L.A., Bengtsson, J., Berggren, Å., Zwiggelaar, K., Spijkman, E., Huyer-Brugman, F. \& Berg, M.P. (2015) Spatially structured environmental filtering of collembolan traits in late successional salt marsh vegetation. Oecologia, 179, 537-549.

Widenfalk, L.A., Malmström, A., Berg, M.P. \& Bengtsson, J. (2016) Small-scale Collembola community composition in a pine forest soil - Overdispersion in functional traits indicates the importance of species interactions. Soil Biology and Biochemistry, 103, 52-62.

Xiong, Y., Gao, Y., Yin, W. \& Luan, Y. (2008) Molecular phylogeny of Collembola inferred from ribosomal RNA genes. Molecular phylogenetics and evolution, 49, 728-735.

Yu, D., Zhang, F., Stevens, M.I., Yan, Q., Liu, M. \& Hu, F. (2016) New insight into the systematics of Tomoceridae (Hexapoda, Collembola) by integrating molecular and morphological evidence. Zoologica Scripta, 45, 286-299. 


\title{
Chapter 2
}

\section{Collembola Phylogeny and Trait Evolution}

Ting-Wen Chen, Jo-Fan Chao, Matty P. Berg, Ina Schaefer, Stefan Scheu

\begin{abstract}
Collembola (springtails) are among the most diverse soil mesofauna. As basal Hexapoda derived from a crustacean ancestor they diversified on land and occupied a wide variety of ecological niches, with distinct morphological and ecological traits among different taxonomic groups. However, information on trait evolution during Collembola diversification is scarce. At least in part this is due to the lack of knowledge on phylogeny across taxonomic levels. In this study, we first reconstructed phylogenetic trees of Collembola from various taxonomic groups that locally co-occur in arable fields, grasslands and forests. We then used phylogenetic comparative methods to investigate the evolution of functional traits of Collembola. Results demonstrate that body shape of Collembola evolved quickly early in their diversification but slowed down afterwards. In contrast, evolutionary transitions of pigmentation, number of ommatidia and reproductive mode correlated with vertical stratification of species living in soils. Ancestral traits of Collembola were likely slender body, hemiedaphic way of life, sexual reproduction, possession of many ommatidia and bright body color, but these traits presumably changed several times during diversification of species. The traits with phylogenetic signal can help to investigate assembly processes in soil animal communities using community phylogenetic approaches and our study paves the way for integrating of evolutionary approaches into soil ecological studies.
\end{abstract}

\section{Keywords}

ancestral state reconstruction; Brownian motion model; Early Burst model; evolutionary constraint; functional trait; life form; phylogenetic comparative method; phylogenetic signal; reproductive mode; springtail; soil 


\section{Introduction}

Diversification processes of soil organisms are less investigated than aboveground biota, albeit the tremendous diversity of soil animals has puzzled ecologists for decades (Anderson 1975, Petersen and Luxton 1982, Giller 1996). Extant soil invertebrates include Nematoda, Annelida, Chelicerata, Myriapoda and Hexapoda that terrestrialized several times independently (von Reumont et al. 2012, Rota-Stabelli et al. 2013, Minter et al. 2017). Among these, Hexapoda derived from a crustacean ancestor with the fossil record dating back to the Devonian or earlier (Hirst and Maulik 1926, Whalley and Jarzembowski 1981, Rota-Stabelli et al. 2013, Wolfe et al. 2016) and rapidly diversified into various ecological niches. Today, Collembola (springtails) are the most abundant and diverse Hexapoda living in soil (Hopkin 1997).

About 8,600 species of Collembola have been described (Bellinger et al. 1996-2017; www.collembola.org). Systematically, they are classified into the four orders: Poduromorpha, Entomobryomorpha, Symphypleona and Neelipleona. Species of different orders are characterized by distinct morphological characters and usually prefer different ecological environments. For example, Entomobryomorpha are elongate in body shape, while Symphypleona are globular, and both usually live on the soil surface. Similarly, elongated Poduromorpha and globular Neelipleona predominantly dwell in the soil (Salmon et al. 2014). Morphological characters possessed by different Collembola species presumably are associated with adaptation to the habitat they colonize, and thus represent functional traits on which ecological processes can work (Violle et al. 2007, Pey et al. 2014).

However, traits associated with environmental factors are also shaped by evolutionary processes. As being inherited from a common ancestor, functional traits of species show phylogenetic signal, i.e., closely related species possess similar traits. As a result of different evolutionary mechanisms, a trait can be phylogenetically conserved (i.e., shaped by evolutionary constraints), convergent (i.e., evolved repeatedly in distantly related species) or labile. These evolutionary mechanisms can be inferred using phylogenetic comparative methods (Blomberg and Garland 2002, Losos 2008, Revell et al. 2008, Cooper et al. 2010) such as phylogenetic signal measurements, ancestral character state reconstruction and likelihood comparisons of evolutionary models. In this study we examined the patterns of evolution in Collembola functional traits including body length, body shape, intensity of pigmentation, number of ommatidia (eyes), vertical stratification in soil profile and reproductive mode-all associated with environmental factors (Widerfalk et al. 2015).

Although it appeals intuitively that variations in functional traits of different species have evolutionary bases, few studies have tested evolutionary hypotheses of traits in soil animals. Previous studies indicated that desiccation resistance of Isopoda (Dias et al. 2013) and defense mechanisms of Oribatida (Pachl et al. 2012) exhibit phylogenetic signal. In Collembola, ecological preferences (Ponge and Salmon 2013) and stable isotopic signatures (Potapov et al. 2016) are likely to exhibit phylogenetic signal. Recently, Chen et al. (2017) demonstrated that fatty acid profiles of Collembola exhibit phylogenetic signal. Furthermore, Malcicka et al. (2017) suggested that trophic guilds of Collembola and their mouthpart structures evolved 
in parallel. However, the results of these studies are limited, as they only used a single comparative method (Dias et al. 2013, Malcicka et al. 2017, Chen et al. 2017) or used taxonomy as proxy for phylogenetic relationships without basing them on phylogenetic trees inferred by genetic markers (Ponge and Salmon 2013, Potapov et al. 2016).

One challenge in applying phylogenetic comparative approaches to soil animals is the lack of phylogenetic information for most taxa. In Collembola phylogenetic relationships between the four orders are still debated. Symphypleona, either paraphyletic (D’Haese 2002, Luan et al. 2005) or monophyletic (Xiong et al. 2008, Schneider et al. 2011, Yu et al. 2016), are recovered as the sister group to the other Collembola. Species of Entomobryomorpha are paraphyletic, presumably due to the position of Tomoceridae. Furthermore, the phylogenetic position of Neelipleona is not resolved, although they are likely more closely related to Entomobryomorpha than to Symphypleona (Deharveng 2004). However, previous studies on Collembola phylogeny were based on few genetic markers (D'Haese 2002), few taxa (Xiong et al. 2008) or focused on a narrow spectrum of taxonomic groups (Frati and Carapelli 1999, Frati et al. 2000, Soto-Adames 2002, Burkhardt and Filser 2005, Park 2009, Cicconardi et al. 2010, Greenslade et al. 2011, Schneider et al. 2011, Cicconardi et al. 2013, Zhang et al. 2014, Yu et al. 2016). A phylogeny of Collembola including all major evolutionary lineages and various taxonomic levels is still lacking.

In this study, we reconstructed phylogenetic trees of Collembola from various taxonomic groups that locally co-occur in arable fields, grasslands and forests in Central Europe. We further included sequences deposited in GenBank for additional Central European species, to construct a more comprehensive phylogeny. Then, we mapped Collembola traits on the phylogenetic tree, measured phylogenetic signal, tested models of trait evolution and reconstructed ancestral states. We hypothesized that (1) Collembola functional traits show phylogenetic signal, with closely related species having similar traits, and that (2) the evolution of major traits of Collembola is correlated with the vertical stratification of species in soil, i.e. their depth distribution.

\section{Materials and Methods}

\section{Taxa sampling}

Collembola were sampled between March and June 2014 from arable fields, grasslands and forests at six sites near Göttingen, Germany (Figure 2.1, Table S2.1). In each of the arable fields and grasslands, one suction sample equal to a surface area of $154 \mathrm{~cm}^{2}$ was taken for $10 \mathrm{~s}$ to collect surface-living individuals. Then, to sample soil-dwelling individuals, a soil core ( $5 \mathrm{~cm}$ diameter, $5 \mathrm{~cm}$ depth) was taken at the center of the area from which the suction sample was collected. In each forest, litter material $(\mathrm{L}$ and $\mathrm{F}$ layer) from an area of $154 \mathrm{~cm}^{2}$ was sampled by hand, followed by a $10 \mathrm{sec}$ suction sample of the humus layer ( $\mathrm{H}$ layer). Animals from this suction sample later were added to the animals from the litter sample forming the full sample of organic layers. Further, to sample soil dwelling Collembola, a soil core $(5 \mathrm{~cm}$ 
diameter, $5 \mathrm{~cm}$ depth) was taken at the center of the area sampled for Collembola in organic layers. Collembola from the suction samples from arable fields and grasslands were directly transferred into $96 \%$ ethanol, while those in the organic layers and soil cores were extracted by heat (Kempson et al. 1963), collected in water and then transferred into $96 \%$ ethanol every two days over a period of ten days. Samples were kept at $4^{\circ} \mathrm{C}$ until identification and then stored at $-80^{\circ} \mathrm{C}$. Collembola identification was based on Hopkin (2007), Fjellberg (1998, 2007) and Gisin (1960); nomenclature followed Bellinger et al. (1996-2017; www.collembola.org).

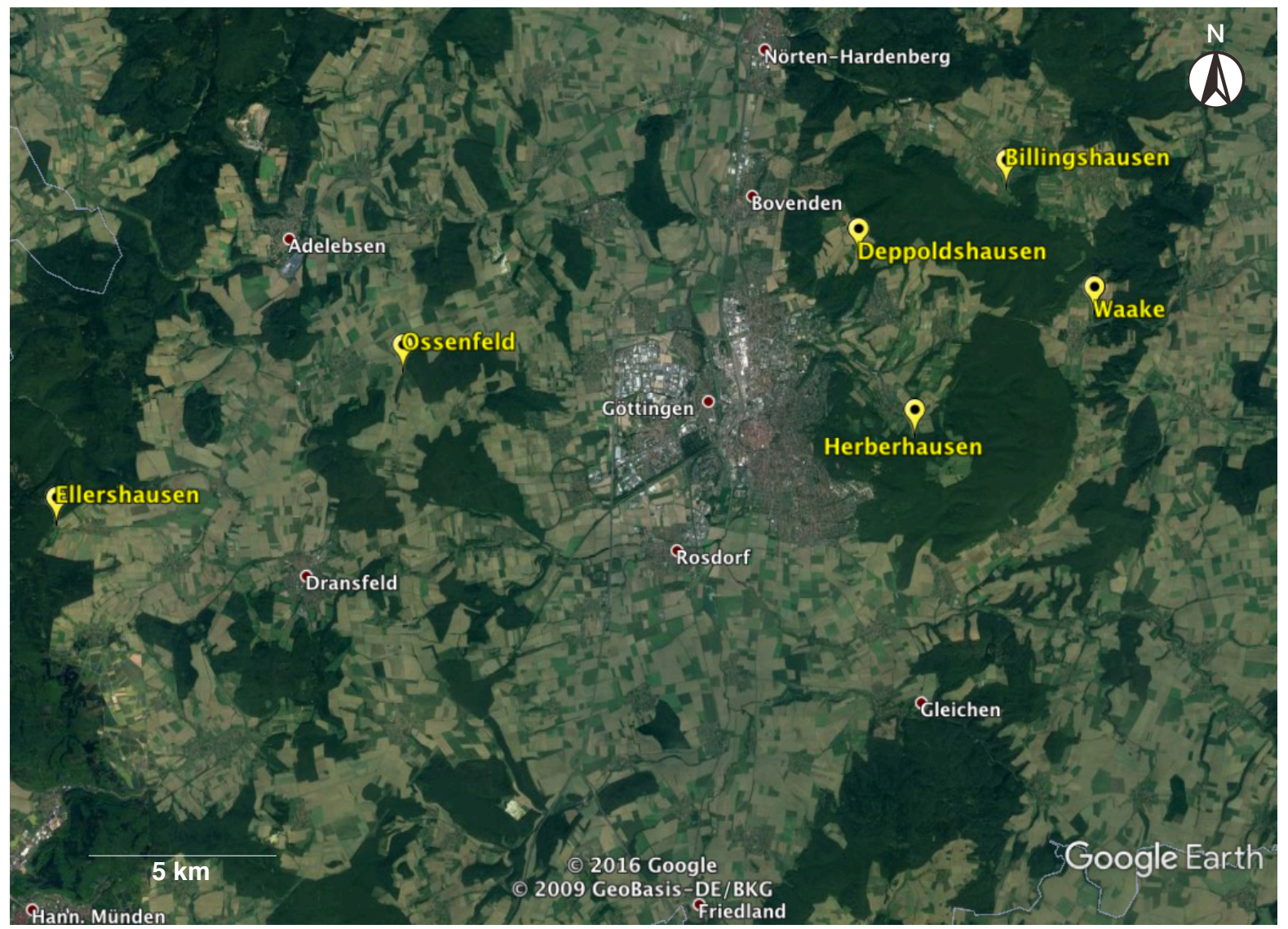

Figure 2.1

Sampling sites of this study. For more details see text and Table S2.1.

\section{DNA extraction, PCR and sequencing}

In total, 75 morphological species representing 51 genera and 18 families were collected from the study sites and used for phylogenetic reconstruction. To cover cryptic species, multiple individuals of each species were sequenced, if replicates were available. Genomic DNA from a single individual was extracted using the DNeasy ${ }^{\circledR}$ Blood and Tissue Kit (Qiagen, Hilden, Germany) following the manufacturer's protocol for animal tissue. Two ribosomal genes, partial $18 \mathrm{~S}$ rRNA ( 680 bp; McGaughran et al. 2010) and partial 28S rRNA covering the D1-D2 region ( 780 bp; D’Haese 2002) and D3-D5 region ( 570 bp; Luan et al. 2005), and two protein coding genes, Histone H3 (H3; nuclear gene, 
372 bp; von Saltzwedel et al. 2016) and Cytochrome Oxidase I (COI; mitochondrial gene, 681 bp; Schneider et al. 2011), were amplified separately in $25 \mu \mathrm{l}$ volumes containing $12.5 \mu \mathrm{l}$ SuperHot Taq Mastermix (Genaxxon Bioscience GmbH, Ulm, Germany), $1.5 \mu$ l of each primer (10 pM; Table S2.2), $2 \mu \mathrm{l} \mathrm{MgCl}_{2}(25 \mathrm{mM})$ and 3-5 $\mu \mathrm{l}$ template DNA. PCR conditions for 18S rRNA, 28S rRNA and H3 included an initial activation step at $95^{\circ} \mathrm{C}$ for $15 \mathrm{~min}$, followed by 35 amplification cycles (with a denaturation step at $94^{\circ} \mathrm{C}$ for $30 \mathrm{~s}$, a primer-annealing step at the optimal temperature for $45 \mathrm{~s}$ and a elongation step at $72^{\circ} \mathrm{C}$ for $30 \mathrm{~s}$; Table S2.2) and ended with a final elongation step at $72^{\circ} \mathrm{C}$ for 6 min. The PCR program for COI was identical to that used in Anslan and Tedersoo (2015). Positive PCR products were purified with the PCR DNA Purification Mini Prep Kit (Genaxxon Bioscience GmbH, Ulm, Germany) following the manufacturer's protocol and sent for sequencing to the Göttingen Genome Laboratory (Institute for Microbiology and Genetics, University of Göttingen, Germany). The obtained sequences were checked and ambiguous positions were corrected using Sequencher 5.0 (Gene Code Corporation, Ann Arbor, Michigan, USA) aided by chromatograms.

\section{Phylogeny of field-collected Collembola}

\section{Individual-based unrooted phylogeny}

First, individual-based phylogenetic trees were built to remove redundant sequences of the same species. Sequences of the five genetic markers were aligned separately in R 3.2.2 (R Core Team 2015) using the functions AlignSeqs and AdjustAlignment with the default parameter settings (package "DECIPHER"; Wright 2015). In each marker set, terminal gaps at the beginning and the end of sequences were replaced by "?". The alignments of the five markers were concatenated in a supermatrix (3,084 bp) using SequenceMatrix 1.8 (Vaidya et al. 2011). An unrooted phylogeny was inferred using Bayesian Inference (BI) in MrBayes 3.2.4 (Ronquist et al. 2012), setting the model of sequence evolution as GTR+I+G. Bayesian Inference was conducted by two independent runs of four chains, 5,000,000 generations, 0.02 temperature and 0.5 burn-in fraction; all other parameters remained set as default. The resulting consensus tree was checked to remove redundant sequences of the same species for the following species-based phylogenetic inference. Redundant sequences were excluded if genetic distances between individuals were less than $5 \%$ across all five markers. Different lineages of the same species were retained if they showed genetic distances more than $5 \%$ using the $\mathrm{R}$ function otuPhylo (Steven Kembel pers. comm.). Overall, a total of 102 operational taxonomic units (OTUs) of different species and withinspecies lineages were obtained for the species-based phylogenetic reconstruction (Table S2.3; Accession Number KY230697-KY231137).

\section{Species-based phylogeny}

Sequences of the 102 OTUs were aligned with the outgroup taxa Zygentoma (Insecta), Machilis (Insecta: Archaeognatha), Callibaetis (Insecta: Palaeoptera), Baculentulus (Protura), Parajapyx (Diplura) and 
Speleonectes (Crustacea; Table S2.3) using the R functions mentioned above. For each genetic marker the aligned sequences were trimmed to the same length. The best model of sequence evolution for all tested markers were fitted with $\mathrm{GTR}+\mathrm{I}+\mathrm{G}$ according to Akaike information criterion (AIC) estimated in jModelTest 2.1.4 (Guindon and Gascuel 2003, Darriba et al. 2012). Terminal gaps of each alignment were replaced by "?", and a supermatrix including all genetic markers was generated using SequenceMatrix 1.8 (Vaidya et al. 2011), resulting in a total length of 3,650 bp of the alignment. Bayesian Inference (BI; MrBayes 3.2.4, Ronquist et al. 2012) was applied for phylogenetic reconstruction by setting the five markers (all GTR $+\mathrm{I}+\mathrm{G}$ ) unlinked, two independent runs, four chains, 10,000,000 generations, 0.05 temperature and 0.5 burn-in fraction. Other parameters were set as default. Furthermore, Collembola phylogeny was inferred using Maximum Likelihood (ML) algorithm in RAxML 7.0.3 (Stamatakis 2006) setting the GTR $+\mathrm{I}+\mathrm{G}$ model and 1,000 bootstrap replicates. BI and ML trees were similar in topology and we continued the analyses of trait evolution with the BI tree.

\section{Extended Collembola phylogeny}

To construct a more comprehensive phylogeny, we further included sequences of the abovementioned genes reported in the literature for other Collembola species with recorded Central European occurrence (Bellinger et al. 1996-2017; www.collembola.org). Here 242 OTUs of 167 species, 82 genera and 18 families were downloaded from NCBI, covering most of the common Collembola in Central Europe (Table S2.4). Since outgroups always resulted in polytomies in the backbone of the tree in preliminary trials, we constructed an unrooted phylogeny using the extended dataset. The downloaded sequences were aligned with the field-collected dataset following the steps mentioned above, except that D1 and D2 regions of $28 \mathrm{~S}$ rRNA were aligned separately and the three codon positions of proteincoding genes (H3 and COI) were spilt into three alignments. The model of sequence evolution of each marker was estimated using jModelTest 2.1.4. Terminal gaps of each alignment were replaced by "?", and concatenated in a supermatrix. The total length of the alignment was 3,083 bp. Bayesian Inference was applied, setting the genetic markers unlinked and other parameters as default except for 10,000,000 generations, 0.02 temperature and 0.5 burn-in fraction.

\section{Ultrametric tree transformation}

The species-based BI tree was transformed to a chronogram (ultrametric tree) using a penalized likelihood approach by setting different models of substitution rate variation among branches, i.e., correlated, relaxed, discrete and strict clock models, using the function chronos implemented in the $\mathrm{R}$ package "ape" (Paradis et al. 2004). The most appropriate ultrametric tree was selected based on the smallest value among all PHIIC generated from different models (Paradis 2013). The ultrametric tree based on the strict clock model was selected. For the morphological species including different genetic 
lineages (cryptic species), only one OTU was retained. Traits of species were mapped onto the ultrametric phylogenetic tree using the function plot.phylo implemented in the R package "ape" (Paradis et al. 2004).

\section{Collembola traits}

Traits of the field-sampled Collembola species were extracted from a trait database (Matty P. Berg, unpublished data) compiled from literature, including maximum body length, overall body shape, intensity of pigmentation, number of ommatidia (eyes), vertical stratification in soil profile and reproductive mode. These traits are associated with environmental gradients or stress (Vandewalle et al. 2010, Makkonen et al. 2011, Salmon et al. 2014, Widenfalk et al. 2015). Collembola body length was used as continuous variable spanning from 0.4 to $6.5 \mathrm{~mm}$, whereas body shape (slender, stocky, spheric), pigmentation (pale, bright, dark), number of ommatidia [many (6-8), few (1-5), none (0)], vertical stratification (epedaphic, hemiedaphic, euedaphic) and reproductive mode (parthenogenetic, bisexual) were used as categorical variables. Species occurrence in each of the three habitats was further included as a binary variable [0 (absence), 1 (presence)], and species logarithmic density summed across habitat types and sites was treated as further continuous variable (Table S2.4).

\section{Phylogenetic signal}

Phylogenetic signal in continuous, categorical and binary variables was analyzed using Blomberg's K (Blomberg et al. 2003), Pagel's lambda (Pagel 1999, Freckleton et al. 2002) and D statistic (Fritz and Purvis 2010), respectively. Blomberg's $\mathrm{K}$ was calculated using the function phylosig implemented in the $\mathrm{R}$ package "phytools" (Blomberg et al. 2003, Revell 2012). Significance tests were done by randomizing species on the phylogeny 1,000 times to test whether trait values showed phylogenetic signal or not (i.e., $\mathrm{H} 0=0$ ). In case of significant $\mathrm{K}$-values of traits, the observed $\mathrm{K}$-value was further compared with 10,000 simulated K-values to test whether phylogenetic signal significantly differed from the level expected under Brownian motion evolution model (i.e., $\mathrm{H} 0=1$; Revell et al. 2007). Simulations of trait values were conducted using the function fastBM in the R package "phytools" (Revell 2012). Lower and higher phylogenetic signal than predicted by a Brownian motion model was defined as a K-value in the 0.025 and 0.975 quantiles of the log-transformed simulated K-values, respectively. For categorical variables, Pagel's lambda was estimated using the function fitDiscrete implemented in $\mathrm{R}$ package "geiger" (Pagel 1999, Freckleton et al. 2002, Harmon et al. 2008). An appropriate trait evolution model was estimated from one of the equal-rates (ER), symmetric (SYM) and all-rates-different (ARD) models using likelihood comparison. The ER model was accepted for all categorical traits. Then, a star-like tree (lambda 0) was transformed from the original tree (lambda 1). Likelihoods of the distribution of trait states among species were compared, given the lambda 1 and lambda 0 trees. If the lambda 0 tree was accepted, the trait showed no phylogenetic signal (Pagel 1999, Freckleton et al. 2002). Phylogenetic signal in binary variables was measured using the $\mathrm{D}$ statistic with 10,000 permutations by the function phylo.d 
implemented in the R package "caper" (Fritz and Purvis 2010, Orme et al. 2013). A D-value smaller than zero indicated a trait with conserved phylogenetic signal, while a value greater than one suggested that a trait is divergent. The observed $\mathrm{D}$-value was compared with simulated $\mathrm{D}$-values generated by two models, phylogenetic randomness and Brownian threshold models (Fritz and Purvis 2010).

\section{Ancestral state estimation}

For continuous traits with phylogenetic signal, ancestral character states were estimated using Maximum Likelihood method by the function fast $A n c$ and then followed by a traitgram plotted using the function phenogram implemented in the R package "phytools" (Revell 2012). For the categorical traits with phylogenetic signal, stochastic character mapping was used to estimate ancestral character states using the function make.simmap implemented in the R package "phytools" (Huelsenbeck et al. 2003, Bollback, 2006, Revell 2012). Trait state transition was assumed following the ER model. Prior distribution on root node was estimated from tip character states. Transition matrix $Q$ was sampled 1,000 times from the posterior probability distribution using Bayesian MCMC. Then, 1,000 stochastic maps were simulated which were conditioned by the sampled value of Q. Numbers of trait state transitions in the tree were calculated based on the mean and median. Posterior probabilities of trait states were mapped to the tree nodes.

\section{Evolution model of traits}

For continuous traits, the fits of trait evolution under Brownian motion model (BM) and OrnsteinUhlenbeck model (OU) were compared using the function fitContinuous implemented in the R package "geiger", while for discrete traits, the fits of trait evolution under the rate constancy model, white-noise model, Pagel's lambda model (lambda), time-dependent model (delta), Early-burst model (EB) and punctuational model (kappa) were compared using the function fitDiscrete. Akaike Weights $\left(\mathrm{AIC}_{\mathrm{w}}\right)$ were used to estimate the support of each tested model to the trait states.

\section{Correlated evolution of traits}

To explore the evolution of traits Collembola body length was converted into a categorical variable and coded as small $(0.4-1.5 \mathrm{~mm})$, medium $(1.6-3.1 \mathrm{~mm})$ and large $(>3.2 \mathrm{~mm})$. Then, all the categorical trait variables were coded as binary variables to estimate relationships of evolutionary changes between any two traits using Pagel's general method as implemented in the function fitPagel in the R package "phytools" (Pagel 1994, Revell 2012). The model of evolution for each trait was set as ER. Using likelihood ratio test, the independent model was compared with the dependent model. Three dependent models (" $x$ ", " $y$ " and "xy") were used to explore the dependency of substitution rate of one variable on the other ("X" on "Y"), that of the opposite direction ("Y" on "X") and that in both directions ("X" on "Y" and "Y" on "X"; Pagel 1994). P-values were derived from 1,000 simulations and then adjusted using Benjamini and Hochberg corrections (BH; Benjamini and Hochberg 1995). 


\section{Results}

\section{Phylogeny of field-collected Collembola}

Both BI tree and ML tree using the data on local Collembola species supported monophyletic origin of major taxonomic groups including Entomobryoidea, Isotomidae, Tomoceridae, Neelidae, Symphypleona and Poduromorpha. Entomobryoidea was sister to all other Collembola lineages. Isotomidae was sister to the remaining taxa, while Poduromorpha formed the sister lineage to Symphypleona. Neelidae and Tomoceridae formed one monophyletic clade positioned between Isotomidae and the clade comprising Poduromorpha and Symphypleona, although this positioning was based on weak support. Within Poduromorpha, Neanuridae plus Hypogastruridae and Brachystomellidae formed a monophyletic clade, sister to the clade composed of Tullbergiidae, Onychiuridae and Odontellidae. Notably, monophyly of Hypogastruridae was not supported, and Brachystomellidae was nested within Neanuridae. Symphypleona comprised two clades, one including Sminthurididae, Arrhopalitidae and Katiannidae, and the other including Bourletiellidae and Sminthuridae (with Sminthurides parvulus, however). The position of Dicyrtomidae in the BI tree (sister to Bourletiellidae + Sminthuridae) differed from that in the ML tree (sister to Sminthurididae + Arrhopalitidae + Katiannidae). In Isotomidae most genera formed monophyletic clades including Parisotoma, Isotoma, Vertagopus and Folsomia; however, species of the genus Desoria were separated, with D. violacea Fjellberg, 1979 forming the sister taxon of Vertagopus and D. trispinata forming the sister taxon of Parisotoma. Within Entomobryoidea, Orchesellidae was sister group to Lepidocyrtidae + Entomobryidae (Figure 2.2).

In addition to the position of Dicyrtomidae, the topology of the ML tree differed from that of the BI tree in the relationships within Tullbergiidae, relationship of Brachystomellidae with the other clades, monophyly of Neelus murinus, the position of Isotomodes productus within Isotomidae and that of Pseudosinella within Lepidocyrtidae (Figure S2.1).

\section{Phylogeny of species occurring in Central Europe}

Including the sequences of the other European Collembola species, the phylogenetic tree showed several monophyletic groups: Poduromorpha, Symphypleona, Tomoceridae, Neelipleona, Isotomidae, and Entomobryoidea. Within Poduromorpha, except Triacanthella perfecta (Hypogastruridae) of which the sequence was taken from GenBank, the monophyly of Onychiuroidea was supported and included three monophyletic families, Onychiuridae, Odontellidae and Tullbergiidae. Odontellidae was the sister group to Tullbergiidae, while Onychiuridae was the sister group to Odontellidae plus Tullbergiidae. Relationships of the remaining Poduromorpha were complex. Both Hypogastruridae and Neanuridae were not monophyletic, while Poduridae and Brachystomellidae were close to Neanuridae. Symphypleona was the sister group to Poduromorpha. In Symphypleona, Sminthurididae formed the sister clade to Katiannoidea which composed of Arrhopalitidae and Katiannidae. Dicyrtomidae was the sister group to 


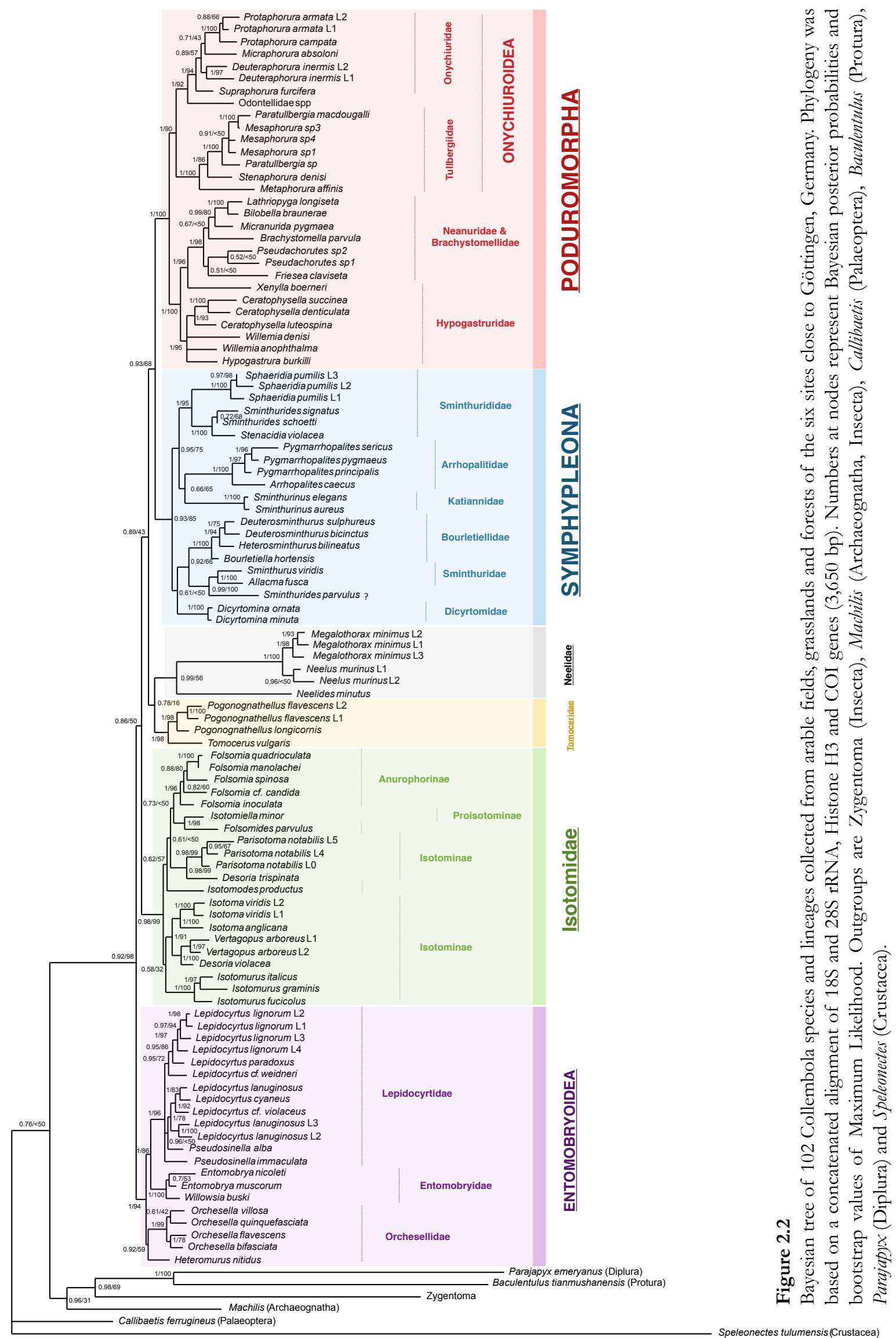


Sminthuroidea which included Sminthuridae and Bourletiellidae. Isotomidae seemed closely related to Entomobryoidea. In Isotomidae, Archisotoma was sister to the other species. Within Entomobryoidea, Entomobryidae and Lepidocyrtidae were sister to each other, forming a monophyletic group sister to Orchesellidae. However, relationships of Tomoceridae and Neelipleona to the other Collembola were not resolved (Figure 2.3).

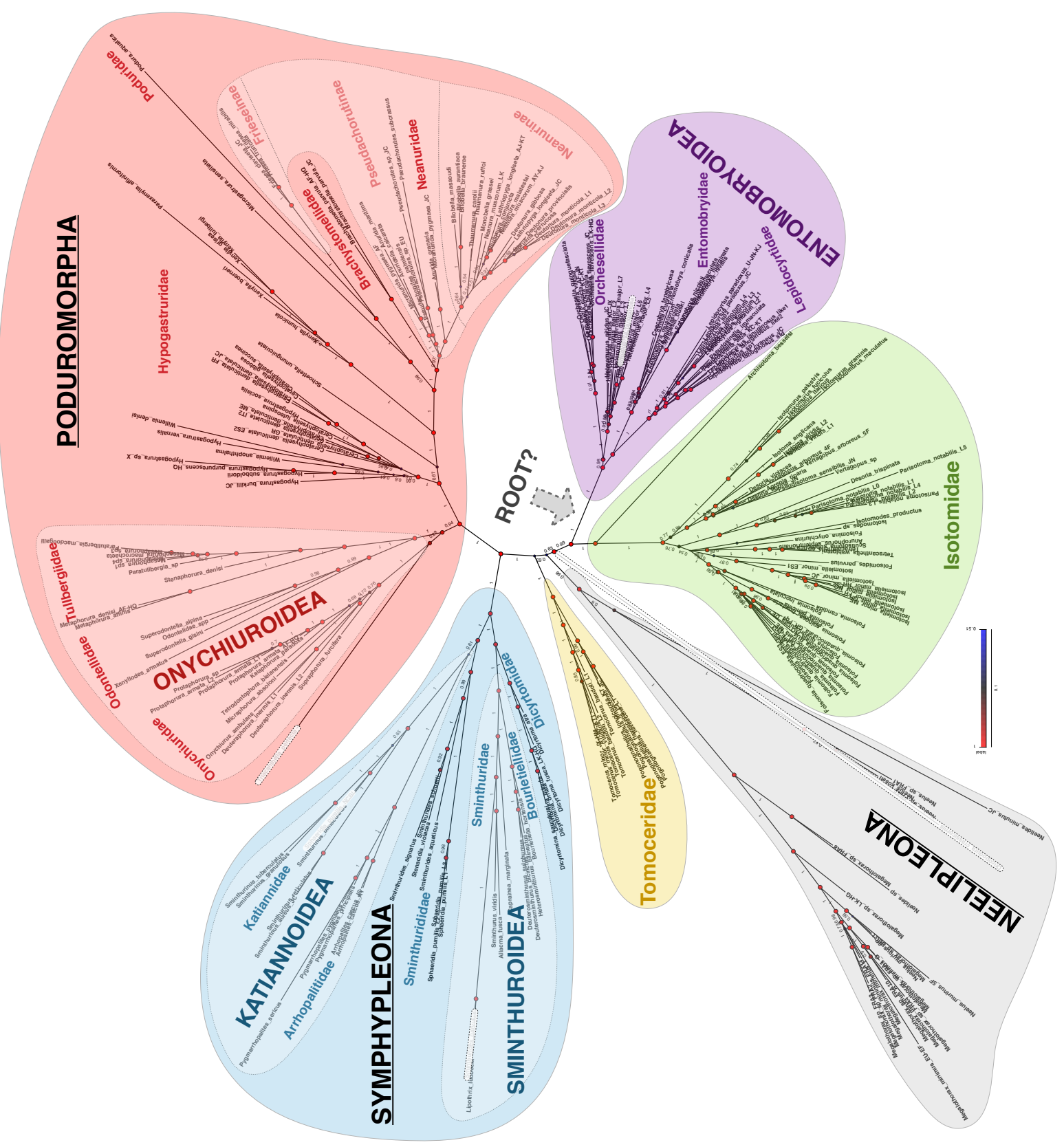

Figure 2.3

Unrooted Bayesian tree of 242 Collembola taxa (including 167 species, 82 genera and 18 families) based on a concatenated alignment of $18 \mathrm{~S}$ and $28 \mathrm{~S}$ rRNA, Histone H3 and COI genes (3,083 bp). Numbers next to branches and colors on nodes represent posterior probabilities of 20,002 sampled trees. 


\section{Phylogenetic signal of traits}

Collembola body length exhibited phylogenetic signal following Brownian motion model $(\mathrm{K}=0.786$, $P_{(\mathrm{H} 0=0)}=0.001 ; 2.5 \%-97.5 \%$ simulated K: 0.587-1.770, $P_{(\mathrm{H} 0=1)}=0.520 ;$ Figure 2.4, S2.2). Body shape, vertical stratification, pigmentation, number of ommatidia and reproductive mode all showed phylogenetic signal as indicated by Pagel's lambda which approached 1.0 (Table 2.1). Species abundance, however, exhibited lower phylogenetic signal than that predicted by Brownian motion model $(\mathrm{K}=0.412$, $P_{(\mathrm{H} 0=0)}=0.029 ; 2.5 \%-97.5 \%$ simulated $\left.\mathrm{K}: 0.581-1.762, P_{(\mathrm{H} 0=1)}<0.001\right)$, indicating abundant species comprised species from different clades. Species occurrence in different types of habitats, however, exhibited no phylogenetic signal, as indicated by the $\mathrm{D}$-values of $0.889,0.790$ and 0.661 for occurrence in arable fields, grasslands and forests, respectively. All D-values deviated from the Brownian threshold model but followed the phylogenetic randomness model.

\section{Table 2.1}

Phylogenetic signal reported as Pagel's lambda with maximum log likelihood test in Collembola categorical traits. Maximum $\log$ likelihood of a trait fit to the given phylogeny $(\log \mathrm{L})$ was tested against that fit to a lambda transformed phylogeny $(\operatorname{logL} 0$, lambda $=0)$. A significant $P$-value in Pagel's lambda test indicates phylogenetic signal in that trait as predicted by the Brownian motion model.

\begin{tabular}{lccccc}
\hline Trait & States & lambda & $\operatorname{logL}$ & $\operatorname{logL0}$ & $\boldsymbol{P}$ \\
\hline Body shape & 3 & 1.000 & -24.48 & -73.36 & $\mathbf{0 . 0 0 0}$ \\
Vertical stratification & 3 & 1.000 & -61.56 & -81.87 & $\mathbf{0 . 0 0 0}$ \\
Pigmentation & 3 & 0.995 & -70.63 & -81.49 & $\mathbf{0 . 0 0 0}$ \\
Number of ommatidia & 3 & 1.000 & -53.40 & -73.36 & $\mathbf{0 . 0 0 0}$ \\
Reproductive mode & 2 & 0.899 & -42.21 & -42.45 & $\mathbf{0 . 0 4 4}$ \\
\hline
\end{tabular}

\section{Trait evolution}

Ancestral states of the examined characters of Collembola were intermediate size, slender body shape, hemiedaphic, bright pigmentation, many ommatidia and sexual reproduction (Figure 2.5, S2.2, S2.3). During Collembola diversification trait states changed several times (Table S2.6). Variation in Collembola body length as a continuous variable was not different from that predicted by the BM model as compared to the OU model, suggesting that difference in body length of Collembola species resulted from a gradual and continuous drift when species diverged. However, evolution of Collembola body shape was supported by the EB model, suggesting that evolution of body shape was faster early in Collembola diversification and decelerated afterwards. The evolution models of the other categorical traits were not different from the rate constancy model (Table 2.2). Evolutionary transitions of Collembola traits depended on each other, especially for vertical stratification, number of ommatidia, pigmentation and reproductive mode, with euedaphic stratification correlated with pale pigmentation, none ommatidia and parthenogenesis (Figure 2.6, S2.4, Table S2.7). Interestingly, body shape did not correlate with any other examined traits. 


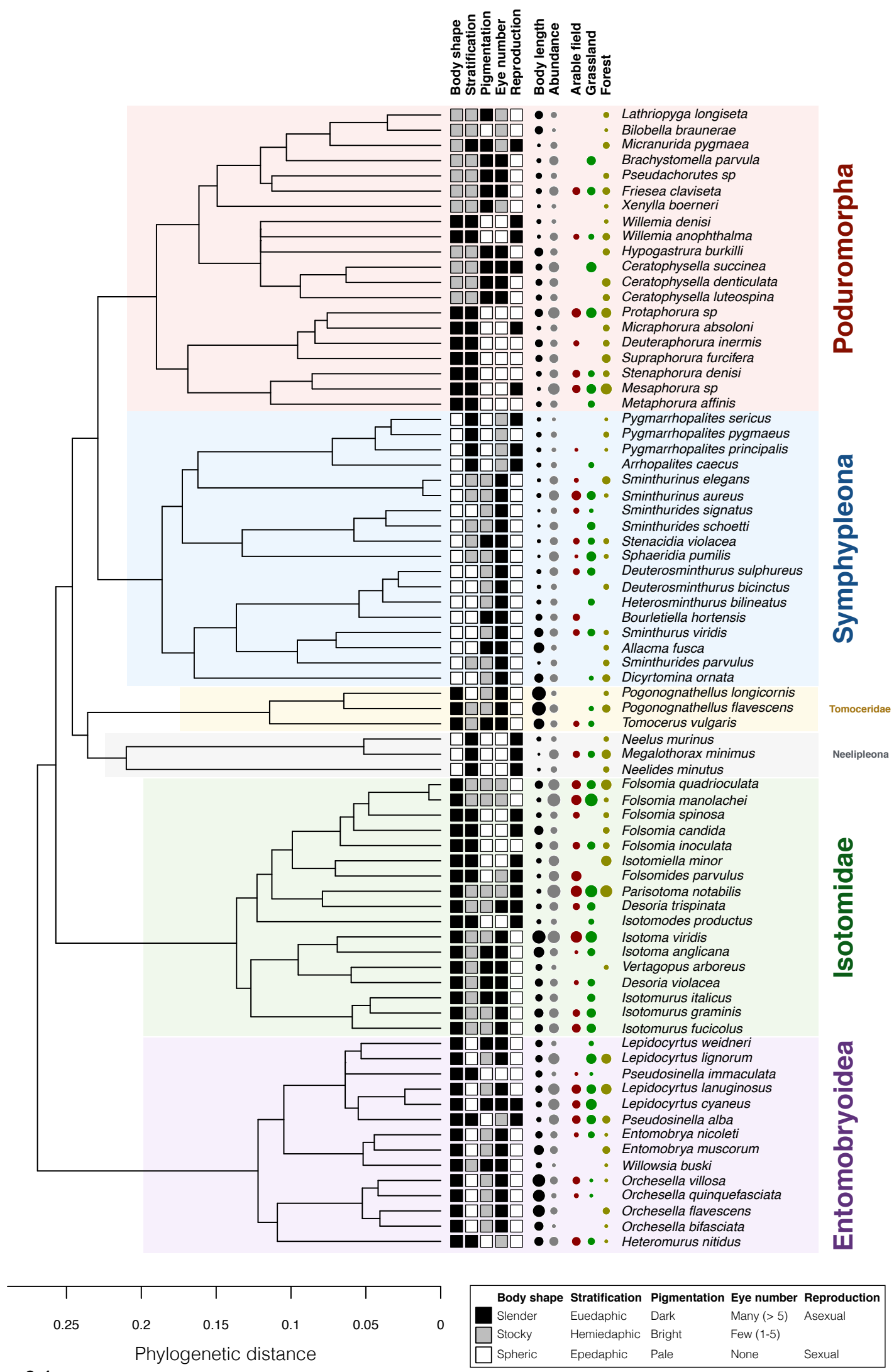

Figure 2.4

Ultrametric phylogenetic tree of the 75 Collembola species based on the Bayesian tree using penalized likelihood that assumes a strict clock model of substitution rate variation among branches. Trait states (squares) and trait values (circles) are plotted next to the tree. Size of a circle represents relative quantity of the trait. 


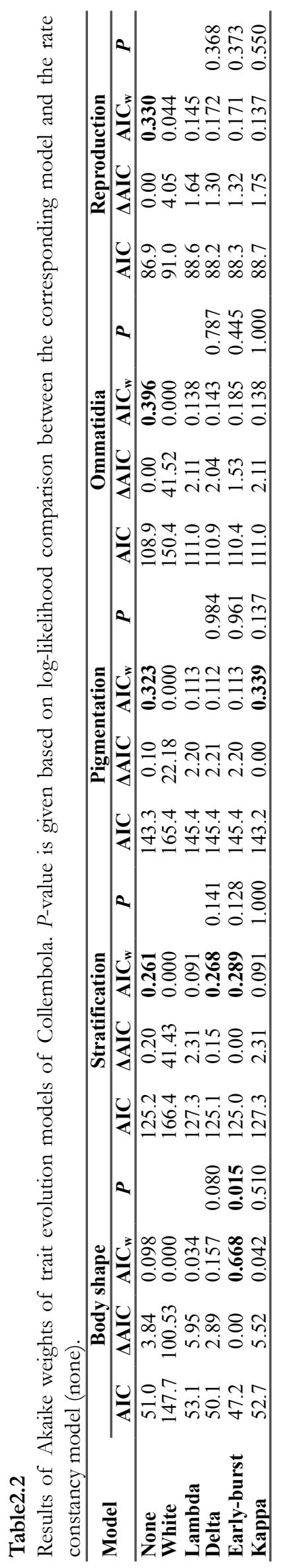




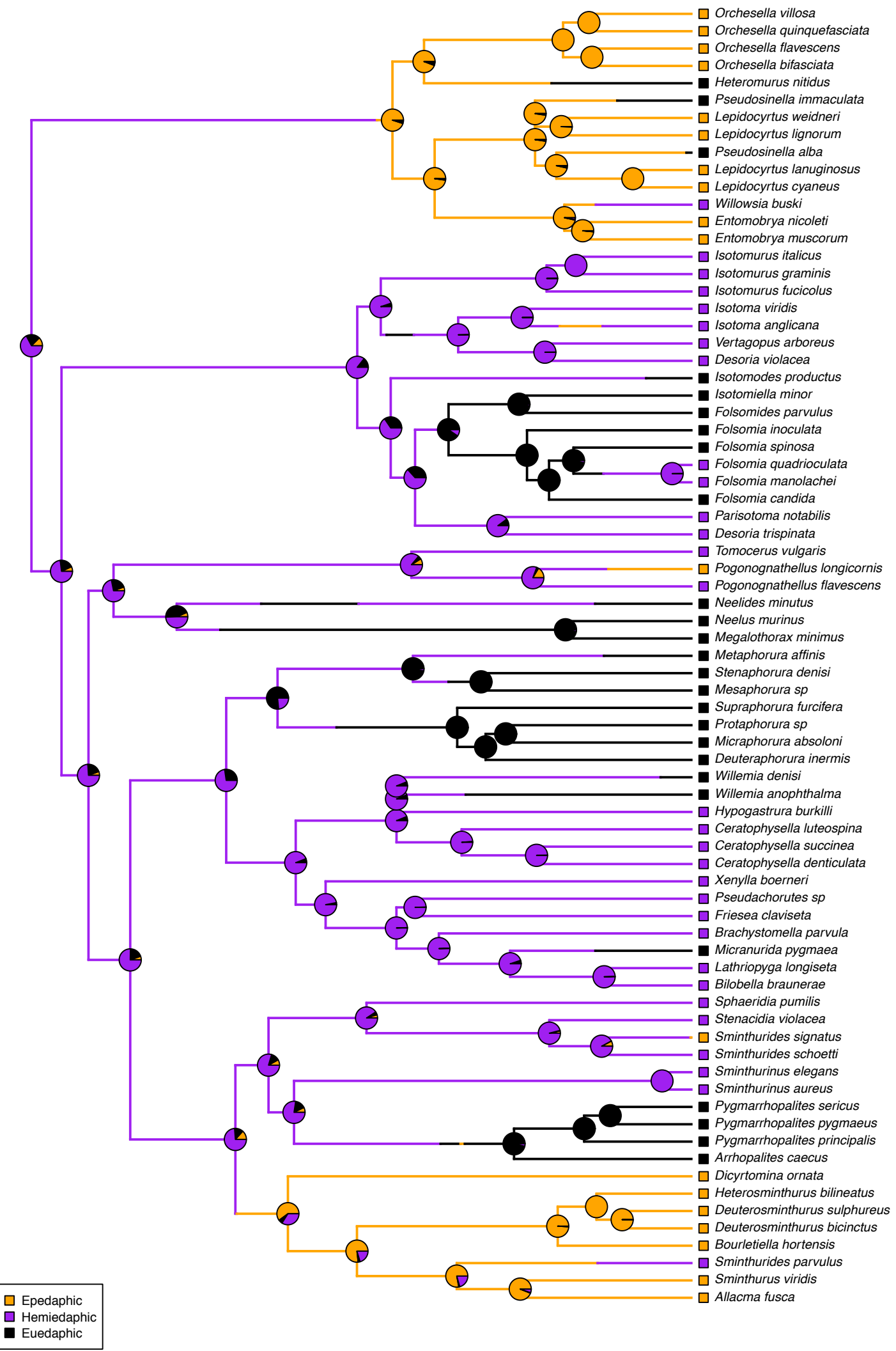

Figure 2.5

Ancestral state reconstruction of vertical stratification of Collembola. Colored squares at the tips of each branch represent the vertical stratification of each extant species. Pie charts on each node indicate the proportion of each character state summed across the posterior distribution of simulations. One of the 1,000 stochastic character maps is plotted. 


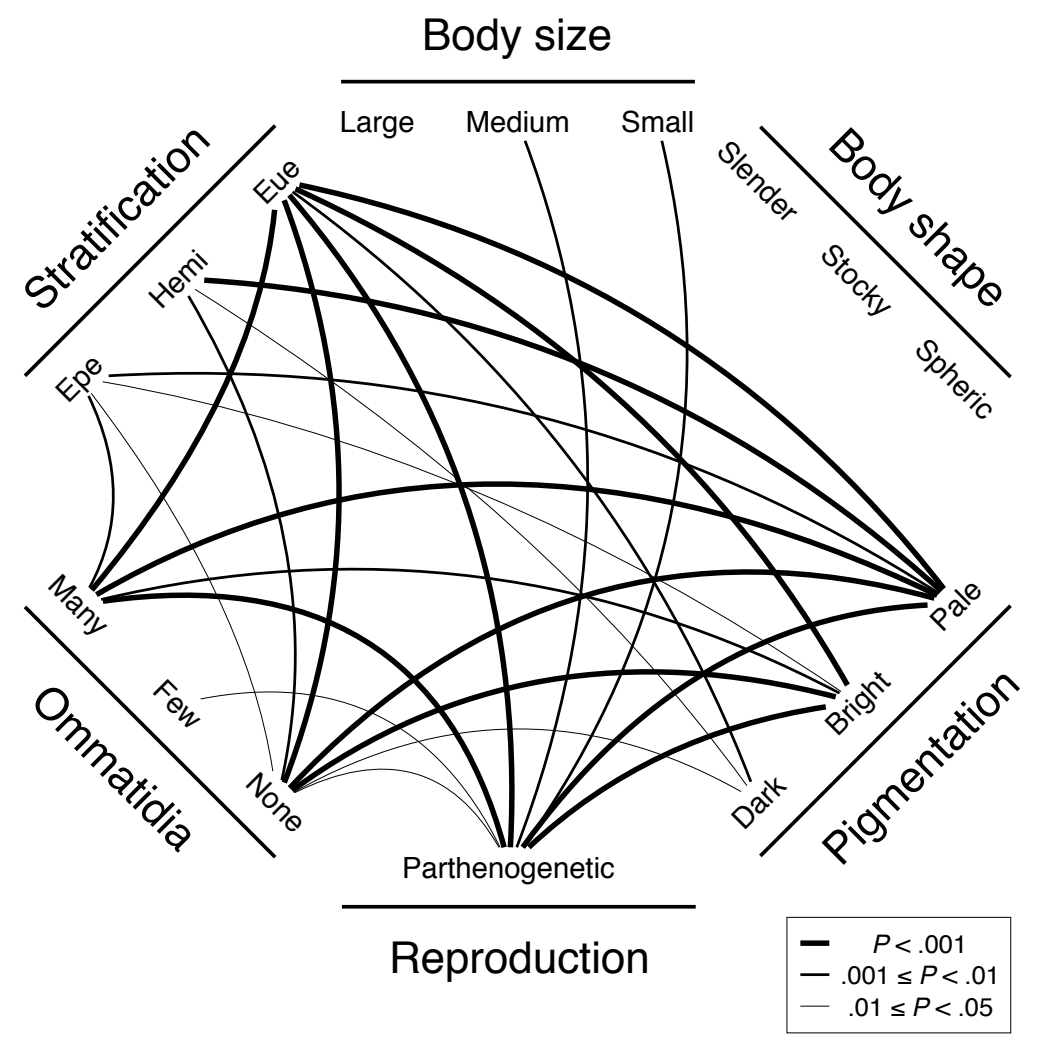

Figure 2.6

Results of Pagel's correlation test between the transition rate of two traits of Collembola; significant dependence between two traits (i.e., "xy" model) are indicated by lines with thickness reflecting the $P$-values. See Table S2.4 for the results of trait dependence by one direction.

\section{Discussion}

In most trait-based studies on soil animals, associations of traits of species with the habitats are usually descriptive but still have been interpreted as the result of adaptation to environmental factors, without testing it in an evolutionary context (Vandewalle et al. 2010, Ponge and Salmon 2012, Bokhorst et al. 2017, but see Malcicka et al. 2017). Here, we conducted phylogenetic comparative analyses to study the evolution of functional traits in soil Collembola. We first reconstructed phylogenetic trees for species occurring in various habitats, and erected a more comprehensive phylogeny as compared to existing Collembola phylogenies. We then focused on the evolution of functional traits of Collembola in light of their associations with different soil layers. Explicitly testing trait evolution is an important step toward integrating phylogeny into studies on assembly processes of soil communities using community phylogenetic approaches. See Chapters 1 and 4 for more details on the community phylogenetic methods.

\section{Collembola phylogeny}

In the present study, both conserved (18S rRNA and H3) and variable (28S rRNA and COI) genetic markers were used to infer the phylogeny of 102 locally occurring Collembola species. Our tree provides 
the most comprehensive Collembola phylogeny to date that included 51 genera and 18 families and considered a variety of closely related outgroups (Misof et al. 2014). This tree indicates that Entomobryoidea and Isotomidae diverged earlier from the other Collembola lineages, while Poduromorpha and Symphypleona are sister groups, contrary to previous studies, which recovered Symphypleona as the sister group to the other Collembola (D'Haese 2002, Xiong et al. 2008, Schneider et al. 2011, Yu et al. 2016). Marker selection, unbalanced taxon sampling and choice of outgroup taxa can affect the topology of phylogenetic trees (Heath et al. 2008, Rosenfeld et al. 2012) and thus resulted in the inconsistency between our tree and those inferred in the previous studies. However, fossil records of Collembola support our findings of the early diverging positions of Entomobryoidea and Isotomidae. The first fossil record of Collembola, Rhyniella praecursor (396-407 million years ago; Hirst and Maulik 1926, Whalley and Jarzembowski 1981, Greenslade 1988), has been assigned to Isotomidae (Greenslade and Walley 1986), suggesting that Isotomidae are likely among the oldest Collembola. Another Paleozoic fossil, Permobrya mirabilis from the Upper Permian, shares characters with extant Entomobryoidea (Riek 1976), suggesting that this superfamily is also phylogenetically old. Fitting the more derived position in our tree, fossil Collembola found in mid and upper Cretaceous amber have been assigned to Collembola families such as Sminthuridae, Neanuridae and Tomoceridae (Christiansen and Pike 2002a, b, Christiansen and Nascimbene 2006). However, in particular early radiation of Collembola lineages needs further investigation using novel approaches, e.g. phylogenomics. Nevertheless, with the extensive taxon sampling and more genes included in this study and the support from the existing fossil record, our tree provides a solid basis for investigating the evolution of traits in Collembola.

\section{Functional traits and their evolution}

Collembola body length, body shape and vertical stratification all showed phylogenetic signal, supporting our first hypothesis. Intuitively plausible, body shape and body length of Collembola may relate to the three-dimensional structure of soil pores (Larsen et al. 2004), as pore size declines typically with soil depth and differs between mineral soil and organic layers. If the structure of soil pores is related to evolutionary adaptation of Collembola species to the vertical distribution in soil profile, we would expect to find evolutionary correlations between body shape, body size and vertical stratification. However, our study does not support the existence of such relationships, suggesting that other mechanisms resulted in the phylogenetic signal of these traits. The evolution of body shape followed the early burst model, suggesting a rapid morphological change in the early evolution followed by relative stasis, presumably under stable evolutionary or ecological constraints. The finding contrasts the conclusion of Harmon et al. (2010) that early evolutionary changes in body shape are rare. In contrast to body shape, body size followed the rate constancy model, suggesting that Collembola gradually changed their body size via drift, an evolutionary process in which trait variation accumulated during evolutionary time. Collembola body size has been shown to relate to desiccation resistance of species (Kærsgaard et al. 2004). Phylogenetic signal detected in the body size therefore supports the idea that phylogenetically 
related species share physiological attributes (Chen et al. 2017). The evolution of vertical stratification of Collembola, though not related to either body shape or body size, was likely related to pigmentation, number of ommatidia and reproductive mode, supporting the second hypothesis.

Evolutionary correlation between pigmentation and vertical stratification suggests that these two traits were functionally related during species diversification. Dark pigmentation is likely a product of adaptation of Collembola to open habitats or surface-living (Salmon et al. 2014), since it reduces damages caused by solar ultraviolet radiation (UV). Also, species with spots and stripes of different colors probably function to mislead predators. Therefore, surface living (epedaphic) species possess shiny or dark pigments, such as many species of Entomobryoidea and Symphypleona. Species dwelling in soil, on the contrary, are usually pale, such as Onychiuroidea. This is further supported by reconstruction of pale coloration as ancestral state in Onychiuroidea. Similar to the pigmentation, significant correlation between the number of ommatidia and vertical stratification indicates that these two traits were functionally related in Collembola evolution. This is also reflected in the eight evolutionary shifts from many ommatidia to no ommatidia, corresponding closely to the nine evolutionary shifts in vertical stratification from hemiedaphic to euedaphic. Surface living Collembola may use their ommatidia as sensory organ to orient themselves according to sunlight (Hågvar 1995) and to detect predators (Salmon et al. 2014). In contrast, euedaphic species rely more on other sensory organs, such as sensory structures on antennae or the postantennal organ (Hopkin 1997, Salmon et al. 2014). Furthermore, contrary to the findings of Malcicka et al. (2017), our study shows that reproductive mode correlated with pigmentation, number of ommatidia and vertical stratification. Indeed, most parthenogenetic Collembola species are euedaphic (Chernova et al. 2010), pale and lack ommatidia. Parthenogenesis in deep soil may reflect the increased difficulty in finding sexual partners due to restricted movement and location of olfactory cues of spermatophores of respectively male partners. In addition, less pronounced resource limitation and dominance of density independent factors in soil may also contribute to the dominance of parthenogenetic Collembola deeper in soil (Chahartaghi et al. 2009).

We did not find phylogenetic signal of species occurrence in different types of habitats, i.e., arable fields, grasslands and forests. Compared to the broadly defined habitats investigated in this study, soil microhabitats, especially the vertical heterogeneity in the soil profile, may have played a more important role for evolutionary adaptations in Collembola than habitat types such as forests and grasslands. Interestingly, in each phylogenetic clade, there was one or few species reaching high abundance, resulting in the significantly lower phylogenetic signal measured in total abundance than that predicted by the Brownian motion model. Since abundance of soil microarthropods positively correlates with the amount of food resources available (Domes et al. 2007, Chahartaghi et al. 2009), this might indicate niche partitioning in food resources between closely related species. Considering the many microhabitats in soil and associated niches, niche partitioning likely contributed to species diversification and this may offer an explanation for the enigma of soil animal biodiversity (Anderson 1975). 


\section{Conclusions and Outlooks}

Overall, phylogenetic signal in body length, body shape, vertical stratification, pigmentation, number of ommatidia and reproductive mode support our first hypothesis that phylogenetically related species of Collembola share similar functional traits. Further, the results suggest that the body shape of Collembola evolved fast during early diversification of lineages but slowed down thereafter. Transitions of pigmentation, number of ommatidia and reproductive mode depended on vertical stratification of species during Collembola diversification, supporting our second hypothesis. The ancestral state of Collembola traits are likely slender body shape, hemiedaphic lifestyle, sexual reproduction, possession of many ommatidia and bright color, but these traits changed several times during Collembola evolution.

Phylogenetic signal in functional traits of soil species provides an evolutionary perspective to soil biodiversity and community assembly processes. The traits of species are products of ecological processes in the past resulting in evolutionary processes. Although trait patterns in communities may reflect environmental associations of the species resulting from contemporary ecological processes, e.g. environmental filtering (Widenfalk et al. 2015), evolution of traits also needs to be considered. If traits evolved in a constrained manner or following Brownian motion model, trait similarity between species in communities cannot be simply inferred as a result of contemporary ecological processes, since also evolutionary mechanisms may have resulted in species possessing similar traits. Our study on trait evolution of soil biota, therefore, paves the way for integrating evolutionary approaches and perspectives into soil ecological studies. Future studies on functional traits and assembly processes of soil communities will benefit from multiple approaches including phylogenetic comparative and community phylogenetic methods.

\section{Author Contributions}

TWC, IS and SS conceived and designed the study; TWC, JFC, and MPB performed the study and collected the data; TWC analyzed the data; TWC, MPB, IS and SS wrote the manuscript.

\section{Data Accessibility}

All DNA sequences generated from this study were deposited in GenBank (Accession Number KY230697-KY231137).

\section{References}

Anderson, J.M. (1975) The enigma of soil animal species diversity. Progress in Soil Zoology: Proceedings of the 5th International Colloquium on Soil Zoology (ed J. Vaněk), pp. 51-58. Springer Netherlands, Dordrecht. 
Anslan, S. \& Tedersoo, L. (2015) Performance of cytochrome c oxidase subunit I (COI), ribosomal DNA Large Subunit (LSU) and Internal Transcribed Spacer 2 (ITS2) in DNA barcoding of Collembola. European Journal of Soil Biology, 69, 1-7.

Bellinger, P.F., Christiansen, K.A. \& Janssens, F. Checklist of the Collembola of the world (www.collembola.org).

Benjamini, Y. \& Hochberg, Y. (1995) Controlling the false discovery rate: a practical and powerful approach to multiple testing. Journal of the Royal Statistical Society Series B, 57, 289-300.

Blomberg, S.P. \& Garland Jr., T. (2002) Tempo and mode in evolution: phylogenetic inertia, adaptation and comparative methods. Journal of Evolutionary Biology, 15, 899-910.

Blomberg, S.P., Garland Jr., T. \& Ives, A.R. (2003) Testing for phylogenetic signal in comparative data: Behavioral traits are more labile. Evolution, 57, 717-745.

Bokhorst, S., Berg, M.P. \& Wardle, D.A. (2017) Micro-arthropod community responses to ecosystem retrogression in boreal forest. Soil Biology and Biochemistry, 110, 79-86.

Bollback, J.P. (2006) SIMMAP: stochastic character mapping of discrete traits on phylogenies. BMC Bioinformatics, 7 , 88.

Burkhardt, U. \& Filser, J. (2005) Molecular evidence for a fourth species within the Isotoma viridis group (Insecta, Collembola). Zoologica Scripta, 34, 177-185.

Carapelli, A., Convey, P., Nardi, F. \& Frati, F. (2014) The mitochondrial genome of the antarctic springtail Folsomotoma octooculata (Hexapoda; Collembola), and an update on the phylogeny of collembolan lineages based on mitogenomic data. Entomologia, 2.

Chahartaghi, M., Maraun, M., Scheu, S. \& Domes, K. (2009) Resource depletion and colonization: A comparison between parthenogenetic and sexual Collembola species. Pedobiologia, 52, 181-189.

Chen, T.-W., Sandmann, P., Schaefer, I. \& Scheu, S. (2017) Neutral lipid fatty acid composition as trait and constraint in Collembola evolution. Ecology and Evolution, 7, 9624-9638.

Chernova, N.M., Potapov, M.B., Savenkova, Y.Y. \& Bokova, A.I. (2010) Ecological significance of parthenogenesis in Collembola. Entomological Review, 90, 23-38.

Christiansen, K. \& Nascimbene, P. (2006) Collembola (Arthropoda, Hexapoda) from the mid Cretaceous of Myanmar (Burma). Cretaceous Research, 27, 318-363.

Christiansen, K. \& Pike, E. (2002a) A preliminary report on the Cretaceous Collembola. Pedobiologia, 46, 267-273.

Christiansen, K. \& Pike, E. (2002b) Cretaceous Collembola (Arthropoda, Hexapoda) from the Upper Cretaceous of Canada. Cretaceous Research, 23, 165-188.

Cicconardi, F., Fanciulli, P.P. \& Emerson, B.C. (2013) Collembola, the biological species concept and the underestimation of global species richness. Molecular Ecology, 22, 5382-96.

Cicconardi, F., Nardi, F., Emerson, B.C., Frati, F. \& Fanciulli, P.P. (2010) Deep phylogeographic divisions and longterm persistence of forest invertebrates (Hexapoda: Collembola) in the North-Western Mediterranean basin. Molecular Ecology, 19, 386-400.

Cooper, N., Jetz, W. \& Freckleton, R.P. (2010) Phylogenetic comparative approaches for studying niche conservatism. Journal of Evolutionary Biology, 23, 2529-2539.

D'Haese, C.A. (2002) Were the first springtails semi-aquatic? A phylogenetic approach by means of 28S rDNA and optimization alignment. Proceedings of the Royal Society of London B, 269, 1143-1151.

Darriba, D., Taboada, G.L., Doallo, R. \& Posada, D. (2012) jModelTest 2: more models, new heuristics and parallel computing. Nature Methods, 9, 772.

Deharveng, L. (2004) Recent advances in Collembola systematics. Pedobiologia, 48, 415-433. 
Dias, A.T.C., Krab, E.J., Mariën, J., Zimmer, M., Cornelissen, J.H.C., Ellers, J., Wardle, D.A. \& Berg, M.P. (2013) Traits underpinning desiccation resistance explain distribution patterns of terrestrial isopods. Oecologia, 172, 667677.

Domes, K., Scheu, S. \& Maraun, M. (2007) Resources and sex: Soil re-colonization by sexual and parthenogenetic oribatid mites. Pedobiologia, 51, 1-11.

Fjellberg, A. (1998) The Collembola of Fennoscandia and Denmark Part I: Poduromorpha (eds NP Kristensen and V Michelsen). Koninklijke Brill, Leiden. The Netherlands.

Fjellberg, A. (2007) The Collembola of Fennoscandia and Denmark Part II: Entomobryomorpha and Symphypleona (eds NP Kristensen and V Michelsen). Koninklijke Brill, Leiden. The Netherlands.

Frati, F. \& Carapelli, A. (1999) An assessment of the value of nuclear and mitochondrial genes in elucidating the origin and evolution of Isotoma klovstadi Carpenter (Insecta, Collembola). Antarctic Science, 11, 160-174.

Frati, F., Dell'Ampio, E., Casasanta, S., Carapelli, A. \& Paolo Fanciulli, P. (2000) Large amounts of genetic divergence among Italian species of the genus Orchesella (Insecta, collembola) and the relationships of two new species. Molecular Phylogenetics and Evolution, 17, 456-61.

Freckleton, R.P., Harvey, P.H. \& Pagel, M. (2002) Phylogenetic analysis and comparative data: a test and review of evidence. The American Naturalist, 160, 712-726.

Fritz, S.A. \& Purvis, A. (2010) Selectivity in mammalian extinction risk and threat types: A new measure of phylogenetic signal strength in binary traits. Conservation Biology, 24, 1042-1051.

Giller, P.S. (1996) The diversity of soil communities, the 'poor man's tropical rainforest'. Biodiversity and Conservation, 5, 135-168.

Gisin, H. (1960) Collembolenfauna Europas. Muséum d’Histoire Naturelle, Geneva.

Greenslade, P.J.M. (1988) Reply to R.A. Crowson's “Comments on Insecta of the Rhynie Chert.” Entomologia Generalis, 13, 115-117.

Greenslade, P., Stevens, M.I., Torricelli, G. \& D’Haese, C. a. (2011) An ancient Antarctic endemic genus restored: Morphological and molecular support for Gomphiocephalus hodgsoni (Collembola: Hypogastruridae). Systematic Entomology, 36, 223-240.

Greenslade, P. \& Whalley, P. (1986) The systematic position of Rhyniella praecursor Hirst and Maulik (Collembola). The earliest known hexapod. Second International Seminar on Apterygota, pp. 319-323. University of Siena, Siena, Italy.

Guindon, S. \& Gascuel, O. (2003) A simple, fast and accurate method to estimate large phylogenies by maximumlikelihood. Systematic Biology, 52, 696-704.

Hågvar, S. (1995) Long distance, directional migration on snow in a forest collembolan, Hypogastrura socialis (Uzel). Acta Zoologica Fennica, 196, 200-205.

Harmon, L.J., Losos, J.B., Jonathan Davies, T., Gillespie, R.G., Gittleman, J.L., Bryan Jennings, W., Kozak, K.H., McPeek, M.A., Moreno-Roark, F., Near, T.J., Purvis, A., Ricklefs, R.E., Schluter, D., Schulte, J.A., Seehausen, O., Sidlauskas, B.L., Torres-Carvajal, O., Weir, J.T. \& Mooers, A.T. (2010) Early bursts of body size and shape evolution are rare in comparative data. Evolution, 64, 2385-2396.

Harmon, L.J., Weir, J.T., Brock, C.D., Glor, R.E. \& Challenger, W. (2008) GEIGER: Investigating evolutionary radiations. Bioinformatics, 24, 129-131.

Heath, T.A., Hedtke, S.M. \& Hillis, D.M. (2008) Taxon sampling and the accuracy of phylogenetic analyses. Journal of Systematics and Evolution, 46, 239-257.

Hirst, S. \& Maulik, S. (1926) On some arthropod remains from the Rhynie Chert (old red sandstone). Geological Magazine, 63, 69-71.

Hopkin, S.P. (1997) Biology of the Springtails (Insecta: Collembola). Oxford University Press, Oxford. 
Hopkin, S.P. (2007) A Key to the Collembola (Springtails) of Britain and Ireland (ed Field Studies Council (FSC)). Shrewsbury.

Huelsenbeck, J.P., Nielsen, R., Bollback, J.P. \& Schultz, T. (2003) Stochastic mapping of morphological characters. Systematic Biology, 52, 131-158.

Kærsgaard, C.W., Holmstrup, M., Malte, H. \& Bayley, M. (2004) The importance of cuticular permeability, osmolyte production and body size for the desiccation resistance of nine species of Collembola. Journal of Insect Physiology, 50, 5-15.

Kempson, D., Lloyd, M. \& Ghelardi, R. (1963) A new extractor for woodland litter. Pedobiologia, 3, 1-21.

Larsen, T., Schjønning, P. \& Axelsen, J. (2004) The impact of soil compaction on euedaphic Collembola. Applied Soil Ecology, 26, 273-281.

Losos, J.B. (2008) Phylogenetic niche conservatism, phylogenetic signal and the relationship between phylogenetic relatedness and ecological similarity among species. Ecology Letters, 11, 995-1003.

Luan, Y., Mallatt, J.M., Xie, R.D., Yang, Y.M. \& Yin, W. (2005) The phylogenetic positions of three basal-hexapod groups (Protura, Diplura, and Collembola) based on ribosomal RNA gene sequences. Molecular Biology and Evolution, 22, 1579-1592.

Macfadyen, A. (1961) Improved funnel-type extractors for soil arthropods. The Journal of Animal Ecology, 30, 171184.

Makkonen, M., Berg, M.P., van Hal, J.R., Callaghan, T. V., Press, M.C. \& Aerts, R. (2011) Traits explain the responses of a sub-arctic Collembola community to climate manipulation. Soil Biology and Biochemistry, 43, 377-384.

Malcicka, M., Berg, M.P. \& Ellers, J. (2017) Ecomorphological adaptations in Collembola in relation to feeding strategies and microhabitat. European Journal of Soil Biology, 78, 82-91.

McGaughran, A., Stevens, M.I. \& Holland, B.R. (2010) Biogeography of circum-Antarctic springtails. Molecular Phylogenetics and Evolution, 57, 48-58.

Minter, N.J., Buatois, L.A., Mángano, M.G., Davies, N.S., Gibling, M.R., MacNaughton, R.B. \& Labandeira, C.C. (2017) Early bursts of diversification defined the faunal colonization of land. Nature Ecology \& Evolution, 1, 175.

Misof, B., Liu, S., Meusemann, K., Peters, R.S., Donath, A., Mayer, C., Frandsen, P.B., Ware, J., Flouri, T., Beutel, R.G., Niehuis, O., Petersen, M., Izquierdo-Carrasco, F., Wappler, T., Rust, J., Aberer, A.J., Aspöck, U., Aspöck, H., Bartel, D., Blanke, A., Berger, S., Böhm, A., Buckley, T.R., Calcott, B., Chen, J., Friedrich, F., Fukui, M., Fujita, M., Greve, C., Grobe, P., Gu, S., Huang, Y., Jermiin, L.S., Kawahara, A.Y., Krogmann, L., Kubiak, M., Lanfear, R., Letsch, H., Li, Y., Li, Z., Li, J., Lu, H., Machida, R., Mashimo, Y., Kapli, P., McKenna, D.D., Meng, G., Nakagaki, Y., Navarrete-Heredia, J.L., Ott, M., Ou, Y., Pass, G., Podsiadlowski, L., Pohl, H., von Reumont, B.M., Schütte, K., Sekiya, K., Shimizu, S., Slipinski, A., Stamatakis, A., Song, W., Su, X., Szucsich, N.U., Tan, M., Tan, X., Tang, M., Tang, J., Timelthaler, G., Tomizuka, S., Trautwein, M., Tong, X., Uchifune, T., Walzl, M.G., Wiegmann, B.M., Wilbrandt, J., Wipfler, B., Wong, T.K.F., Wu, Q., Wu, G., Xie, Y., Yang, S., Yang, Q., Yeates, D.K., Yoshizawa, K., Zhang, Q., Zhang, R., Zhang, W., Zhang, Y., Zhao, J., Zhou, C., Zhou, L., Ziesmann, T., Zou, S., Li, Y., Xu, X., Zhang, Y., Yang, H., Wang, J., Wang, J., Kjer, K.M. \& Zhou, X. (2014) Phylogenomics resolves the timing and pattern of insect evolution. Science, 346, 763-767.

Orme, D., Freckleton, R.P., Thomas, G., Petzoldt, T., Fritz, S.A., Isaac, N. \& W. Pearse. (2013) Caper: comparative analyses of phylogenetics and evolution in R.

Pachl, P., Domes, K., Schulz, G., Norton, R., Scheu, S., Schaefer, I. \& Maraun, M. (2012) Convergent evolution of defense mechanisms in oribatid mites (Acari, Oribatida) shows no "ghosts of predation past." Molecular Phylogenetics and Evolution, 65, 412-420.

Pagel, M. (1994) Detecting Correlated Evolution on Phylogenies: a General Method for the Comparative Analysis of Discrete Characters. Proceedings of the Royal Society of London, 255, 37-45.

Pagel, M. (1999) Inferring the historical patterns of biological evolution. Nature, 401, 877-884. 
Paradis, E. (2013) Molecular dating of phylogenies by likelihood methods: A comparison of models and a new information criterion. Molecular Phylogenetics and Evolution, 67, 436-444.

Paradis, E., Claude, J. \& Strimmer, K. (2004) APE: Analyses of phylogenetics and evolution in R language. Bioinformatics, 20, 289-290.

Park, K.-H. (2009) Molecular biological study on speciation and phylogeny of the order Entomobryomorpha (Collembola: Hexapoda). Entomological Research, 39, 334-340.

Pey, B., Nahmani, J., Auclerc, A., Capowiez, Y., Cluzeau, D., Cortet, J., Decaëns, T., Deharveng, L., Dubs, F., Joimel, S., Briard, C., Grumiaux, F., Laporte, M.-A.A., Pasquet, A., Pelosi, C., Pernin, C., Ponge, J.-F., Salmon, S., Santorufo, L. \& Hedde, M. (2014) Current use of and future needs for soil invertebrate functional traits in community ecology. Basic and Applied Ecology, 15, 194-206.

Ponge, J.-F. \& Salmon, S. (2013) Spatial and taxonomic correlates of species and species trait assemblages in soil invertebrate communities. Pedobiologia, 56, 129-136.

Potapov, A.A., Semenina, E.E., Korotkevich, A.Y., Kuznetsova, N.A. \& Tiunov, A. V. (2016) Connecting taxonomy and ecology: Trophic niches of collembolans as related to taxonomic identity and life forms. Soil Biology and Biochemistry, 101, 20-31.

R Core Team. (2015) R: A language and environment for statistical computing.

von Reumont, B.M., Jenner, R.A., Wills, M.A., Dell'Ampio, E., Pass, G., Ebersberger, I., Meyer, B., Koenemann, S., Iliffe, T.M., Stamatakis, A., Niehuis, O., Meusemann, K. \& Misof, B. (2012) Pancrustacean phylogeny in the light of new phylogenomic data: Support for Remipedia as the possible sister group of Hexapoda. Molecular Biology and Evolution, 29, 1031-1045.

Revell, L.J. (2012) phytools: An R package for phylogenetic comparative biology (and other things). Methods in Ecology and Evolution, 3, 217-223.

Revell, L., Harmon, L. \& Collar, D. (2008) Phylogenetic signal, evolutionary process, and rate. Systematic Biology, 57, 591-601.

Revell, L.J., Johnson, M.A., Schulte, J.A., Kolbe, J.J. \& Losos, J.B. (2007) A phylogenetic test for adaptive convergence in rock-dwelling lizards. Evolution, 61, 2898-2912.

Riek, E.F. (1976) An entomobryid collembolan (Hexapoda: Collembola) from the Lower Permian of southern Africa. Palaeontologia Africana, 19, 141-143.

Ronquist, F., Teslenko, M., van der Mark, P., Ayres, D.L., Darling, A., Höhna, S., Larget, B., Liu, L., Suchard, M.A. \& Huelsenbeck, J.P. (2012) Mrbayes 3.2: Efficient Bayesian phylogenetic inference and model choice across a large model space. Systematic Biology, 61, 539-542.

Rosenfeld, J.A., Payne, A. \& DeSalle, R. (2012) Random roots and lineage sorting. Molecular Phylogenetics and Evolution, 64, 12-20.

Rota-stabelli, O., Daley, A.C. \& Pisani, D. (2013) Molecular timetrees reveal a Cambrian colonization of land and a new scenario for Ecdysozoan evolution. Current Biology, 23, 392-398.

Salmon, S. \& Ponge, J.-F. (2012) Species traits and habitats in springtail communities: A regional scale study. Pedobiologia, 55, 295-301.

Salmon, S., Ponge, J.-F., Gachet, S., Deharveng, L., Lefebvre, N. \& Delabrosse, F. (2014) Linking species, traits and habitat characteristics of Collembola at European scale. Soil Biology and Biochemistry, 75, 73-85.

von Saltzwedel, H., Scheu, S. \& Schaefer, I. (2016) Founder events and pre-glacial divergences shape the genetic structure of European Collembola species. BMC Evolutionary Biology, 16, 148.

Schneider, C., Cruaud, C. \& D’Haese, C.A. (2011) Unexpected diversity in Neelipleona revealed by molecular phylogeny approach (Hexapoda, Collembola). Soil Organisms, 83, 383-398. 
Soto-Adames, F.N. (2002) Molecular phylogeny of the Puerto Rican Lepidocyrtus and Pseudosinella (Hexapoda: Collembola), a validation of Yoshii's “color pattern species". Molecular Pbylogenetics and Evolution, 25, $27-42$.

Stamatakis, A. (2006) RAxML-VI-HPC: Maximum likelihood-based phylogenetic analyses with thousands of taxa and mixed models. Bioinformatics, 22, 2688-2690.

van Straalen, N.M., Timmermans, M.J.T.N., Roelofs, D. \& Berg, M.P. (2008) Apterygota in the spotlights of ecology, evolution and genomics. European Journal of Soil Biology, 44, 452-457.

Vaidya, G., Lohman, D.J. \& Meier, R. (2011) SequenceMatrix: Concatenation software for the fast assembly of multi-gene datasets with character set and codon information. Cladistics, 27, 171-180.

Vandewalle, M., de Bello, F., Berg, M.P., Bolger, T., Dolédec, S., Dubs, F., Feld, C.K., Harrington, R., Harrison, P.A., Lavorel, S., Silva, P.M., Moretti, M., Niemelä, J., Santos, P., Sattler, T., Sousa, J.P., Sykes, M.T., Vanbergen, A.J. \& Woodcock, B.A. (2010) Functional traits as indicators of biodiversity response to land use changes across ecosystems and organisms. Biodiversity and Conservation, 19, 2921-2947.

Violle, C., Navas, M.L., Vile, D., Kazakou, E., Fortunel, C., Hummel, I. \& Garnier, E. (2007) Let the concept of trait be functional! Oikos, 116, 882-892.

Whalley, P. \& Jarzembowski, E.A. (1981) A new assessment of Rhyniella, the earliest known insect, from the Devonian of Rhynie, Scotland. Nature, 291, 317.

Widenfalk, L.A., Bengtsson, J., Berggren, A., Zwiggelaar, K., Spijkman, E., Huyer-Brugman, F. \& Berg, M.P. (2015) Spatially structured environmental filtering of collembolan traits in late successional salt marsh vegetation. Oecologia, 179, 537-549.

Wolfe, J.M., Daley, A.C., Legg, D.A. \& Edgecombe, G.D. (2016) Fossil calibrations for the arthropod Tree of Life. Earth-Science Reviews, 160, 43-110.

Wright, E.S. (2015) DECIPHER: harnessing local sequence context to improve protein multiple sequence alignment. BMC Bioinformatics, 16, 322.

Xiong, Y., Gao, Y., Yin, W. \& Luan, Y. (2008) Molecular phylogeny of Collembola inferred from ribosomal RNA genes. Molecular Phylogenetics and Evolution, 49, 728-735.

Yu, D., Zhang, F., Stevens, M.I., Yan, Q., Liu, M. \& Hu, F. (2016) New insight into the systematics of Tomoceridae (Hexapoda, Collembola) by integrating molecular and morphological evidence. Zoologica Scripta, 45, 286-299.

Zhang, F., Chen, Z., Dong, R.-R., Deharveng, L., Stevens, M.I., Huang, Y.-H. \& Zhu, C.-D. (2014) Molecular phylogeny reveals independent origins of body scales in Entomobryidae (Hexapoda: Collembola). Molecular Phylogenetics and Evolution, 70, 231-9. 


\section{Supplementary Materials}

Table S2.1

Sampling locations of this study.

\begin{tabular}{llll}
\hline Site & Habitat & Latitude $\left({ }^{\circ} \mathbf{N}\right)$ & Longitude $\left({ }^{\circ} \mathbf{E}\right)$ \\
\hline 1 Herberhausen & Arable & 51.53408 & 10.00058 \\
& Grassland & 51.53294 & 9.99273 \\
2 Deppoldshausen & Forest & 51.53072 & 9.99066 \\
& Arable & 51.57565 & 9.97312 \\
& Grassland & 51.57612 & 9.97195 \\
$\mathbf{4}$ Ossenfeld & Forest & 51.57506 & 9.97443 \\
& Arable & 51.54789 & 9.79804 \\
$\mathbf{5}$ Waake & Grassland & 51.54731 & 9.79733 \\
& Forest & 51.54898 & 9.80041 \\
\multirow{3}{*}{ Billingshausen } & Arable & 51.56308 & 10.05845 \\
& Grassland & 51.56293 & 10.06232 \\
& Forest & 51.55943 & 10.07080 \\
8 Ellershausen & Arable & 51.59006 & 10.02655 \\
& Grassland & 51.59372 & 10.03101 \\
& Forest & 51.59235 & 10.03254 \\
& Arable & 51.51264 & 9.66830 \\
& Grassland & 51.50857 & 9.66414 \\
& Forest & 51.51325 & 9.66628 \\
\hline
\end{tabular}

Table S2.2

Primer pairs used in this study.

\begin{tabular}{|c|c|c|c|c|}
\hline Region & Primer & Sequence $5^{\prime}-3^{\prime}$ & Annealing temperature & Reference \\
\hline \multirow[t]{2}{*}{ 18S rRNA } & 18SA2_F & ATGGTTGCAAAGCTGAAAC & $50^{\circ} \mathrm{C}$ & Whiting 2002 \\
\hline & 18S9_R & GATCCTTCCGCAGGTTCACCTAC & & \\
\hline \multicolumn{5}{|l|}{ 28S rRNA } \\
\hline \multirow[t]{2}{*}{ D1-D2 } & $\mathrm{C} 1{ }^{\prime} \mathrm{F}$ & ACCCGCTGAATTTAAGCAT & $50^{\circ} \mathrm{C}$ & D'Haese 2002 \\
\hline & D2coll_R & ACCACGCATGCWTTAGATTG & & \\
\hline \multirow[t]{2}{*}{ D3-D5 } & 28SA_F & GACCCGTCTTGAAGCACG & $52^{\circ} \mathrm{C}$ & Tully et al. 2006 \\
\hline & 28Sbout_R & CCCACAGCGCCAGTTCTGCTTACC & & \\
\hline \multirow[t]{2}{*}{ Histone $\mathrm{H} 3$} & $\mathrm{H} 3 \mathrm{~F} 2$ & ATGGCTCGTACCAAGCAGAC & $56^{\circ} \mathrm{C}$ & Colgan et al. 1998 \\
\hline & H3R2 & ATRTCCTTGGGCATGATTGTTAC & & \\
\hline \multirow[t]{2}{*}{$\mathrm{COI}$} & LCO1490_F & GGTCAACAAATCATAAAGATATTGG & $45^{\circ} \mathrm{C}$ (5 cycles) followed & Folmer et al. 1994 \\
\hline & $\mathrm{HCO} 2198 \_\mathrm{R}$ & TAAACTTCAGGGTGACCAAAAAATCA & by $51^{\circ} \mathrm{C}$ ( 35 cycles $)$ & \\
\hline \multicolumn{5}{|l|}{ References } \\
\hline \multicolumn{5}{|c|}{$\begin{array}{l}\text { Colgan, D.J., McLauchlan, a., Wilson, G.D.F., Livingston, S.P., Edgecombe, G.D., Macaranas, J., Cassis, G. \& Gray, M.R. (1998) Histone } \\
\text { H3 and U2 snRNA DNA sequences and arthropod molecular evolution. Australian Journal of Zoology, 46, } 419 .\end{array}$} \\
\hline \multicolumn{5}{|c|}{$\begin{array}{l}\text { D'Haese, C.A. (2002) Were the first springtails semi-aquatic? A phylogenetic approach by means of } 28 \mathrm{~S} \text { rDNA and optimization } \\
\text { alignment. Proceedings of the Royal Society of London B, 269, 1143-1151. }\end{array}$} \\
\hline \multicolumn{5}{|c|}{$\begin{array}{l}\text { Folmer, O., Black, M., Hoeh, W., Lutz, R. \& Vrijenhoek, R. (1994) DNA primers for amplification of mitochondrial cytochrome c oxidase } \\
\text { subunit I from diverse metazoan invertebrates. Molecular Marine Biology and Biotechnology, 3, 294-299. }\end{array}$} \\
\hline \multicolumn{5}{|c|}{$\begin{array}{l}\text { Tully, T., D'Haese, C.A., Richard, M. \& Ferrière, R. (2006) Two major evolutionary lineages revealed by molecular phylogeny in the } \\
\text { parthenogenetic collembola species Folsomia candida. Pedobiologia, 50, 95-104. }\end{array}$} \\
\hline \multicolumn{5}{|c|}{$\begin{array}{l}\text { Whiting, M.F. (2002) Mecoptera is paraphyletic: Multiple genes and phylogeny of Mecoptera and Siphonaptera. Zoologica Scripta, 31, } \\
\text { 93-104. }\end{array}$} \\
\hline
\end{tabular}




\section{Table S2.3}

NCBI Accession Numbers of sequences generated in this study to construct the molecular phylogeny of Collembola.

\begin{tabular}{|c|c|c|c|c|c|c|c|}
\hline \multirow{2}{*}{\multicolumn{2}{|c|}{ Taxonomic group Family }} & \multirow{2}{*}{ Species } & \multirow{2}{*}{ 18S rRNA } & \multicolumn{2}{|c|}{ 28S rRNA } & \multirow{2}{*}{ Histone H3 } & \multirow{2}{*}{ COI } \\
\hline & & & & D1-D2 & D3-D5 & & \\
\hline \multicolumn{8}{|l|}{ Outgroups } \\
\hline \multicolumn{2}{|l|}{ Crustacea } & Speleonectes & EU370431 & & EU370446 & КС989979 & JF297647 \\
\hline \multicolumn{2}{|l|}{ Protura } & Baculentulus & AY037169 & & EF192433 & & HQ882817 \\
\hline \multicolumn{2}{|l|}{ Diplura } & Parajapyx & AY037168 & & EF192440 & & JQ796635 \\
\hline \multicolumn{2}{|c|}{ Archaeognatha (Insecta) } & Machilis & AY338689 & & AY338646 & AY338614 & JF826083 \\
\hline \multicolumn{2}{|c|}{ Palaeoptera (Insecta) } & Callibaetis & AF370791 & & AY859557 & AY749703 & GU711466 \\
\hline \multicolumn{2}{|c|}{ Zygentoma (Insecta) } & Zygentoma & EU368615 & & EU376048 & AY555568 & JN970940 \\
\hline \multicolumn{8}{|l|}{$\underline{\text { Collembola }}$} \\
\hline \multirow[t]{29}{*}{ Poduromorpha } & Brachystomellidae & Brachystomella parvula & KY230724 & KY230822 & KY230925 & KY231017 & KY231088 \\
\hline & Hypogastruridae & Ceratophysella denticulata & KY230747 & KY230847 & KY230948 & KY231036 & KY231107 \\
\hline & & Ceratophysella luteospina & KY230762 & KY230859 & KY230962 & & KY231118 \\
\hline & & Ceratophysella succinea & & KY230885 & KY230990 & KY231065 & KY231136 \\
\hline & & Hypogastrura burkilli & KY230725 & KY230823 & KY230926 & KY231018 & KY231089 \\
\hline & & Willemia anophthalma & KY230726 & KY230869 & KY230972 & & KY231090 \\
\hline & & Willemia denisi & KY230757 & KY230855 & KY230957 & & KY231114 \\
\hline & & Xenylla boerneri & KY230728 & KY230824 & & & \\
\hline & Neanuridae & Bilobella braunerae & KY230721 & KY230819 & KY230922 & & \\
\hline & & Friesea claviseta & KY230730 & KY230826 & KY230929 & KY231020 & KY231092 \\
\hline & & Lathriopyga longiseta & KY230722 & KY230820 & KY230923 & & KY231086 \\
\hline & & Micranurida pygmaea & KY230723 & KY230821 & KY230924 & & KY231087 \\
\hline & & Pseudachorutes spl & KY230754 & & KY230954 & & KY231113 \\
\hline & & Pseudachorutes sp2 & & KY230886 & & KY231067 & \\
\hline & Odontellidae & Odontellidae spp & KY230796 & & KY230998 & KY231074 & \\
\hline & Onychiuridae & Deuteraphorura inermis L1 & KY230761 & KY230858 & KY230961 & KY231068 & KY231117 \\
\hline & & Deuteraphorura inermis L2 & KY230791 & KY230887 & KY230991 & & \\
\hline & & Micraphorura absoloni & & KY230868 & KY230971 & KY231049 & KY231127 \\
\hline & & Protaphorura armata L1 & KY230759 & KY230856 & KY230959 & KY231064 & KY231116 \\
\hline & & Protaphorura armata L2 & KY230788 & KY230884 & KY230988 & KY231063 & \\
\hline & & Protaphorura campata & KY230770 & KY230867 & KY230970 & KY231048 & KY231126 \\
\hline & & Supraphorura furcifera & KY230792 & KY230888 & KY230992 & KY231069 & KY231085 \\
\hline & Tullbergiidae & Mesaphorura spl & KY230786 & KY230828 & KY230931 & KY231022 & \\
\hline & & Mesaphorura sp3 & KY230785 & KY230882 & KY230987 & KY231062 & \\
\hline & & Mesaphorura sp4 & KY230760 & KY230857 & KY230960 & & \\
\hline & & Metaphorura affinis & KY230731 & KY230827 & KY230930 & KY231021 & KY231093 \\
\hline & & Paratullbergia macdougalli & KY230790 & KY230817 & KY230920 & & \\
\hline & & Paratullbergia sp & KY230787 & KY230883 & & & \\
\hline & & Stenaphorura denisi & KY230720 & KY230818 & KY230921 & & KY231135 \\
\hline \multirow[t]{10}{*}{ Symphypleona } & Arrhopalitidae & Arrhopalites caecus & KY230780 & KY230879 & KY230981 & KY231060 & \\
\hline & & Pygmarrhopalites principalis & & KY230800 & KY230901 & KY231002 & \\
\hline & & Pygmarrhopalites pygmaeus & KY230746 & KY230846 & KY230947 & & KY231106 \\
\hline & & Pygmarrhopalites sericus & & KY230799 & KY230900 & & \\
\hline & Bouletiellidae & Bourletiella hortensis & KY230700 & & KY230898 & & \\
\hline & & Deuterosminthurus bicinctus & KY230732 & KY230829 & KY230932 & KY231023 & KY231094 \\
\hline & & Deuterosminthurus sulphureus & KY230733 & KY230830 & KY230933 & KY231024 & KY231095 \\
\hline & & Heterosminthurus bilineatus & KY230734 & KY230831 & KY230934 & KY231025 & KY231096 \\
\hline & Dicyrtomidae & Dicyrtomina minuta & KY230769 & KY230866 & KY230969 & KY231047 & KY231125 \\
\hline & & Dicyrtomina ornata & KY230768 & KY230865 & KY230968 & KY231046 & \\
\hline
\end{tabular}


Table S2.3 Continued

\begin{tabular}{|c|c|c|c|c|c|c|c|}
\hline \multirow{2}{*}{ Taxonomic group } & \multirow{2}{*}{ Family } & \multirow{2}{*}{ Species } & \multirow{2}{*}{ 18S rRNA } & \multicolumn{2}{|c|}{ 28S rRNA } & \multirow{2}{*}{ Histone $\mathbf{H 3}$} & \multirow{2}{*}{ COI } \\
\hline & & & & D1-D2 & D3-D5 & & \\
\hline & Katiannidae & Sminthurinus aureus & KY230741 & KY230839 & KY230942 & KY231031 & KY231102 \\
\hline & & Sminthurinus elegans & KY230782 & KY230881 & KY230984 & KY231061 & \\
\hline & Sminthuridae & Allacma fusca & KY230735 & KY230832 & KY230935 & & KY231097 \\
\hline & & Sminthurus viridis & KY230701 & KY230798 & KY230899 & KY231001 & \\
\hline & Sminthurididae & Sminthurides parvulus & KY230699 & KY230797 & KY230897 & KY231000 & \\
\hline & & Sminthurides schoetti & KY230781 & & KY230982 & & \\
\hline & & Sminthurides signatus & KY230697 & & KY230895 & KY230999 & \\
\hline & & Sphaeridia pumilis L1 & KY230783 & & KY230985 & & KY231133 \\
\hline & & Sphaeridia pumilis L2 & KY230758 & & KY230958 & & KY231115 \\
\hline & & Sphaeridia pumilis L3 & KY230698 & & KY230896 & & \\
\hline & & Stenacidia violacea & KY230784 & & KY230986 & & KY231134 \\
\hline \multirow[t]{6}{*}{ Neelipleona } & Neelidae & Megalothorax minimus L1 & KY230793 & KY230889 & KY230993 & & \\
\hline & & Megalothorax minimus L2 & & KY230890 & & KY231070 & \\
\hline & & Megalothorax minimus L3 & KY230794 & KY230891 & KY230994 & KY231071 & \\
\hline & & Neelides minutus & KY230716 & KY230813 & KY230916 & & \\
\hline & & Neelus murinus L1 & & KY230892 & KY230995 & KY231072 & \\
\hline & & Neelus murinus L2 & KY230745 & KY230844 & & & KY231104 \\
\hline \multirow[t]{4}{*}{ Tomoceridae } & Tomoceridae & Pogonognathellus flavescens L1 & KY230717 & KY230814 & KY230917 & KY231016 & KY231083 \\
\hline & & Pogonognathellus flavescens L2 & KY230718 & KY230816 & KY230919 & KY231075 & KY231084 \\
\hline & & Pogonognathellus longicornis & & KY230845 & KY230946 & KY231035 & KY231105 \\
\hline & & Tomocerus vulgaris & KY230777 & KY230877 & KY230979 & KY231058 & KY231131 \\
\hline \multirow[t]{21}{*}{ Isotomidae } & Isotomidae & Desoria trispinata & KY230773 & KY230874 & KY230976 & KY231054 & \\
\hline & & Desoria violacea & KY230736 & KY230833 & KY230936 & KY231026 & KY231098 \\
\hline & & Folsomia cf. candida & KY230756 & KY230854 & KY230956 & KY231042 & \\
\hline & & Folsomia inoculata & KY230743 & KY230842 & KY230944 & KY231033 & \\
\hline & & Folsomia manolachei & KY230706 & KY230803 & KY230906 & KY231007 & KY231077 \\
\hline & & Folsomia quadrioculata & KY230755 & KY230853 & KY230955 & & \\
\hline & & Folsomia spinosa & KY230707 & KY230804 & KY230907 & KY231008 & \\
\hline & & Folsomides parvulus & KY230742 & KY230841 & KY230943 & KY231032 & \\
\hline & & Isotoma anglicana & KY230703 & KY230801 & KY230903 & KY231004 & KY231076 \\
\hline & & Isotoma viridis $L 1$ & KY230752 & KY230852 & KY230953 & KY231041 & KY231112 \\
\hline & & Isotoma viridis $L 2$ & KY230774 & KY230875 & KY230977 & KY231055 & KY231129 \\
\hline & & Isotomiella minor & KY230744 & KY230843 & KY230945 & KY231034 & KY231103 \\
\hline & & Isotomodes productus & KY230709 & KY230805 & KY230908 & KY231009 & \\
\hline & & Isotomurus fucicolus & KY230737 & KY230834 & KY230937 & KY231027 & KY231099 \\
\hline & & Isotomurus graminis & KY230738 & KY230836 & KY230939 & & KY231100 \\
\hline & & Isotomurus italicus & KY230739 & KY230837 & KY230940 & KY231029 & KY231101 \\
\hline & & Parisotoma notabilis LO & KY230772 & KY230872 & KY230974 & KY231052 & KY231128 \\
\hline & & Parisotoma notabilis L4 & KY230771 & KY230870 & KY230973 & KY231050 & \\
\hline & & Parisotoma notabilis L5 & & KY230871 & & KY231051 & \\
\hline & & Vertagopus arboreus L1 & KY230705 & KY230802 & KY230905 & KY231006 & \\
\hline & & Vertagopus arboreus L2 & KY230775 & KY230876 & KY230978 & KY231056 & KY231130 \\
\hline \multirow[t]{5}{*}{ Entomobryoidea } & Entomobryidae & Entomobrya muscorum & KY230710 & KY230806 & KY230909 & KY231010 & \\
\hline & & Entomobrya nicoleti & KY230740 & KY230838 & KY230941 & KY231030 & KY231120 \\
\hline & & Willowsia buski & KY230767 & KY230864 & KY230967 & & KY231124 \\
\hline & Lepidocyrtidae & Lepidocyrtus cyaneus & KY230751 & KY230851 & KY230952 & KY231040 & KY231111 \\
\hline & & Lepidocyrtus lanuginosus & KY230765 & KY230862 & KY230965 & KY231044 & KY231123 \\
\hline
\end{tabular}


Table S2.3 Continued

\begin{tabular}{llllllll}
\hline \multirow{2}{*}{ Taxonomic group Family } & Species & \multirow{2}{*}{ 28S rRNA } & Histone H3 & \multirow{2}{*}{ COI } \\
\cline { 4 - 6 } & & Lepidocyrtus lanuginosus L2 & KY230748 & KY230848 & KY230949 & KY231037 & KY231108 \\
& Lepidocyrtus lanuginosus L3 & KY230763 & KY230860 & KY230963 & & KY231121 \\
& Lepidocyrtus lignorum L1 & KY230764 & KY230861 & KY230964 & KY231043 & KY231122 \\
& Lepidocyrtus lignorum L2 & KY230766 & KY230863 & KY230966 & KY231045 & \\
& Lepidocyrtus lignorum L3 & KY230750 & KY230850 & KY230951 & KY231039 & KY231110 \\
& Lepidocyrtus lignorum L4 & KY230749 & KY230849 & KY230950 & KY231038 & KY231109 \\
& Lepidocyrtus paradoxus & KY230795 & KY230894 & KY230996 & KY231073 & \\
& Lepidocyrtus cf. violaceus & KY230778 & KY230878 & KY230980 & KY231059 & KY231132 \\
& Lepidocyrtus cf. weidneri & & KY230893 & & & KY231137 \\
& Pseudosinella alba & KY230711 & KY230807 & KY230910 & KY231011 & \\
& Pseudosinella immaculata & KY230712 & & KY230911 & KY231012 & KY231078 \\
& Heteromurus nitidus & KY230776 & KY230808 & KY230912 & KY231013 & KY231079 \\
& Orchesella bifasciata & KY230713 & KY230810 & KY230914 & & KY231081 \\
& Orchesella flavescens & KY230714 & KY230811 & KY230915 & KY231015 & KY231082 \\
& Orchesella quinquefasciata & & KY230809 & KY230913 & KY231014 & KY231080 \\
& Orchesella villosa & KY230729 & KY230825 & KY230928 & KY231019 & KY231091 \\
\hline
\end{tabular}




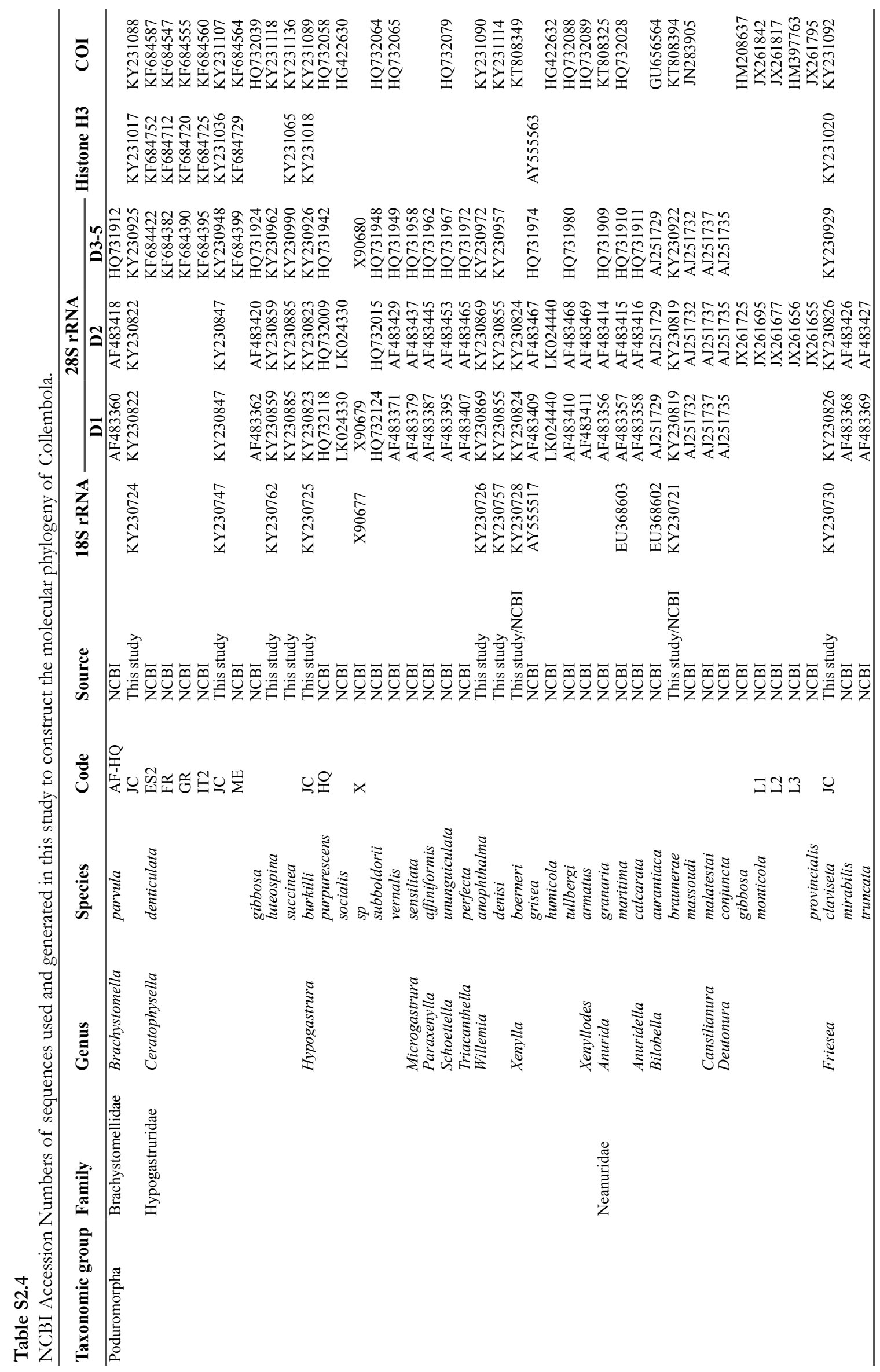




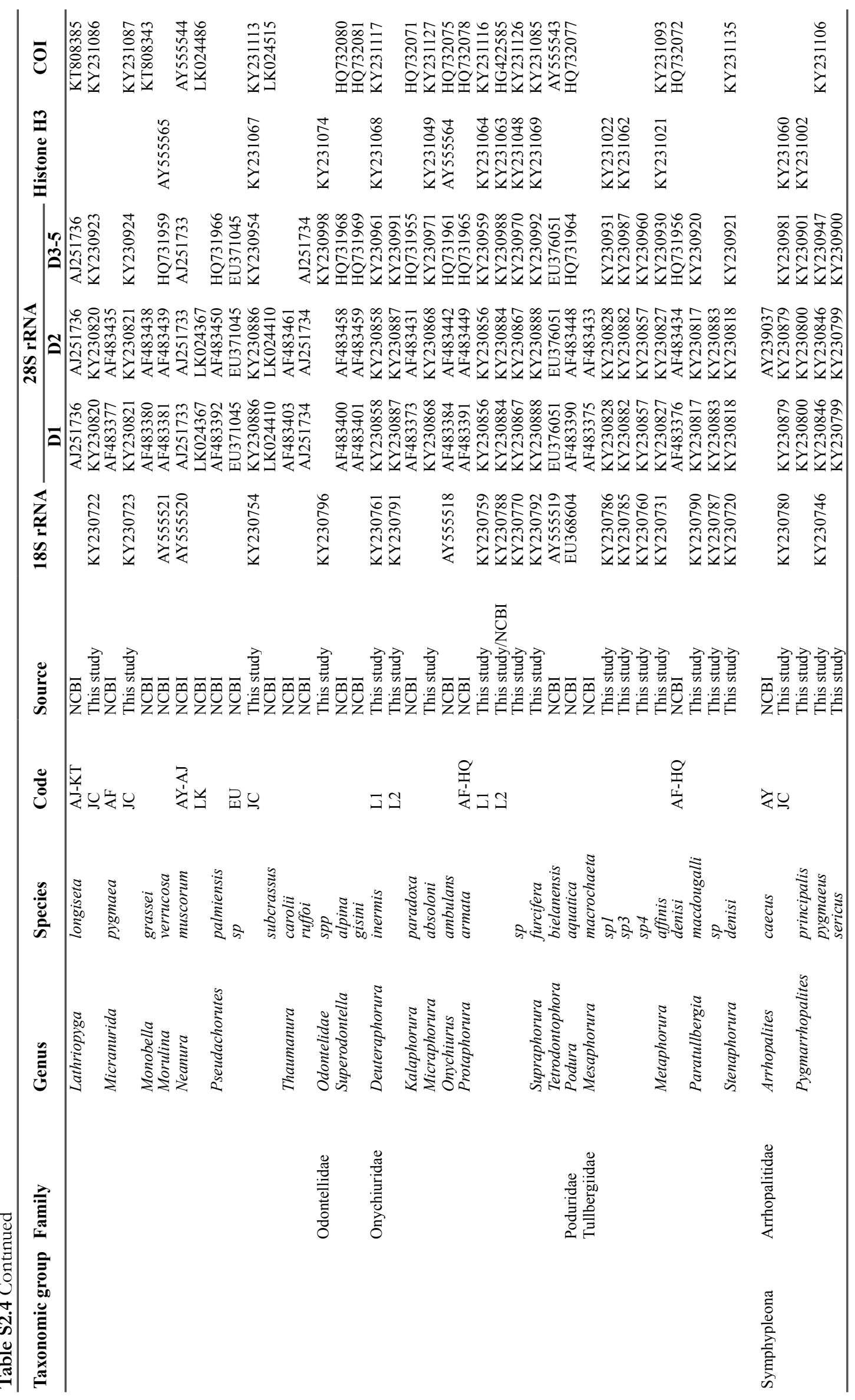




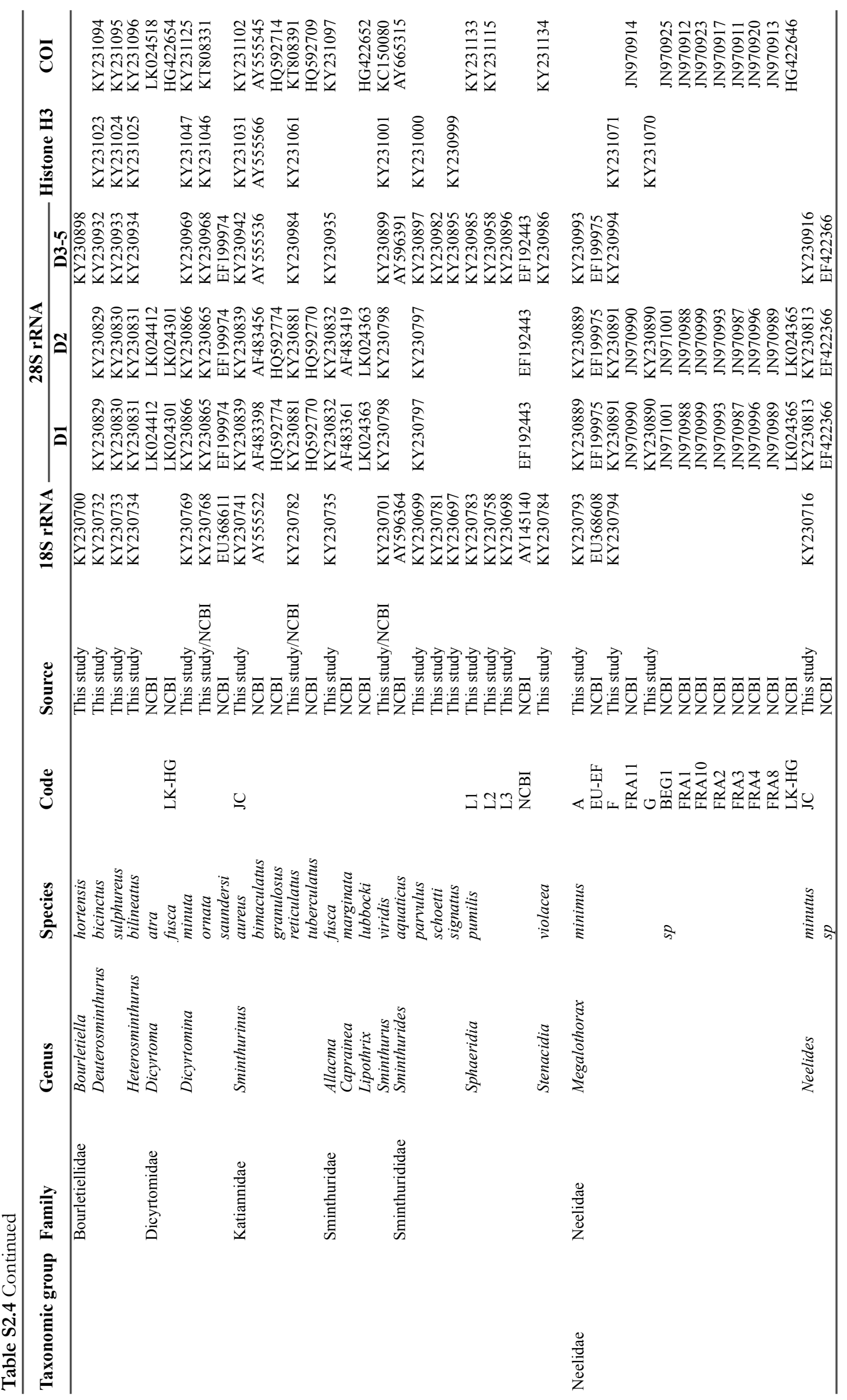




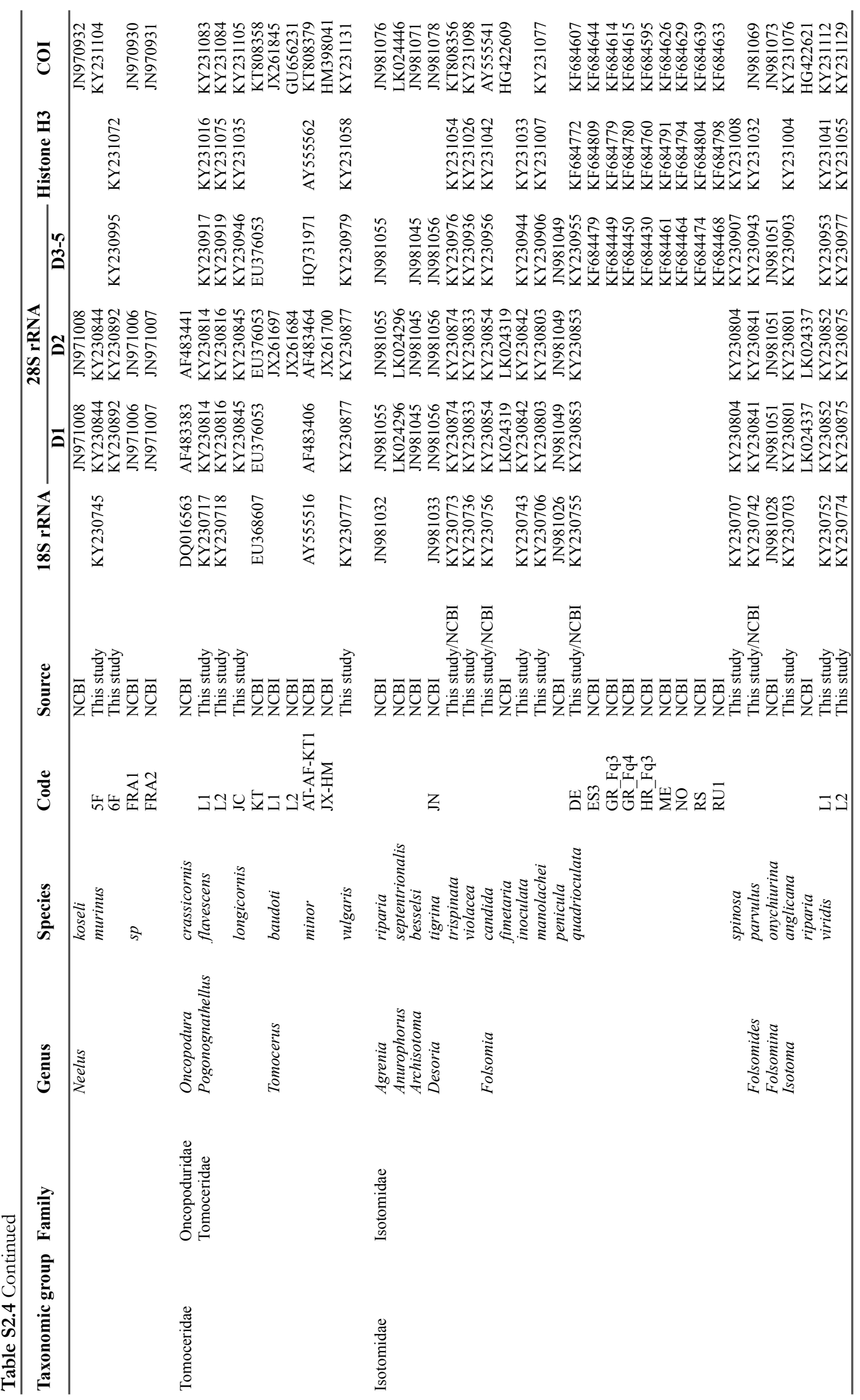




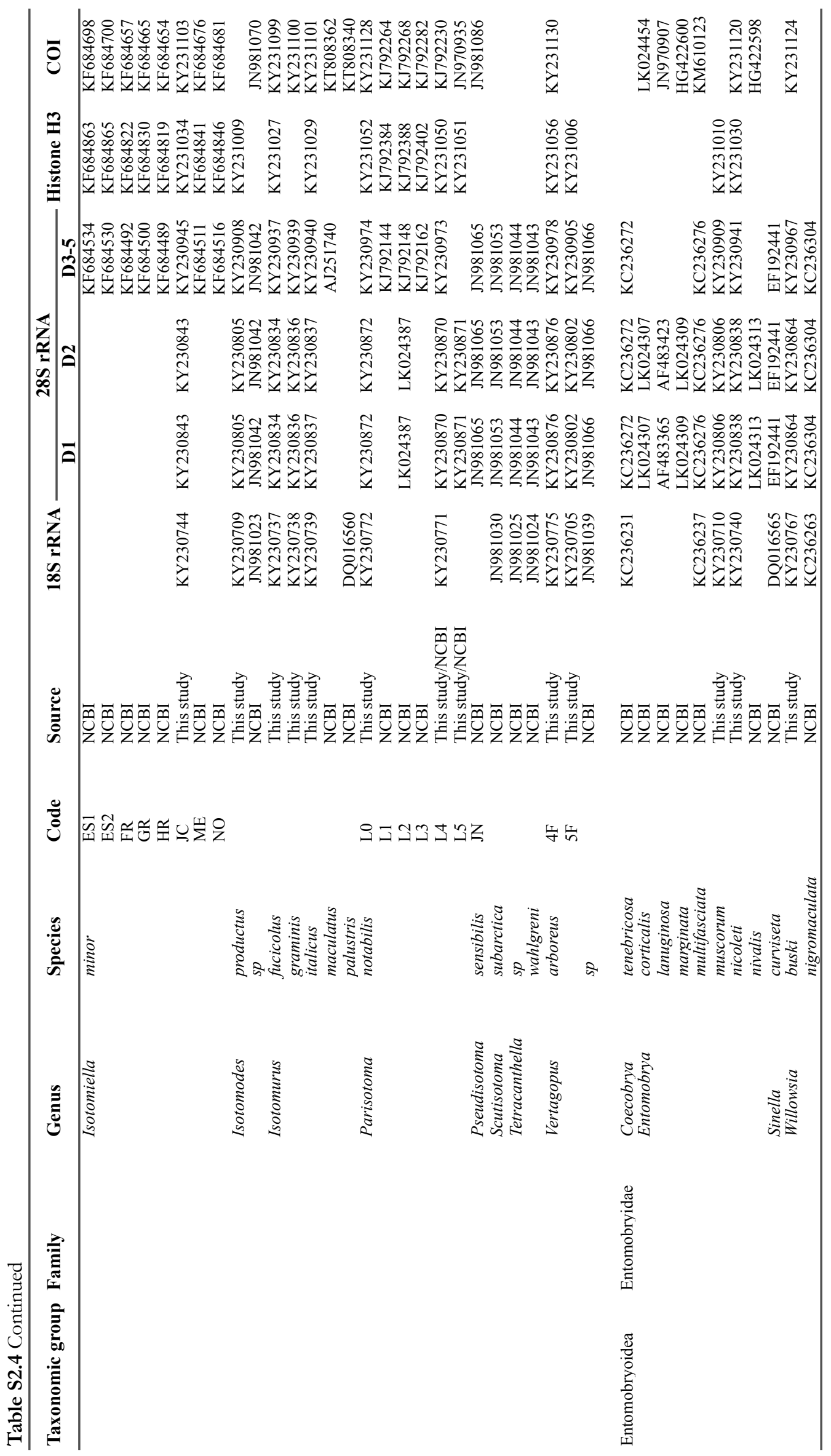




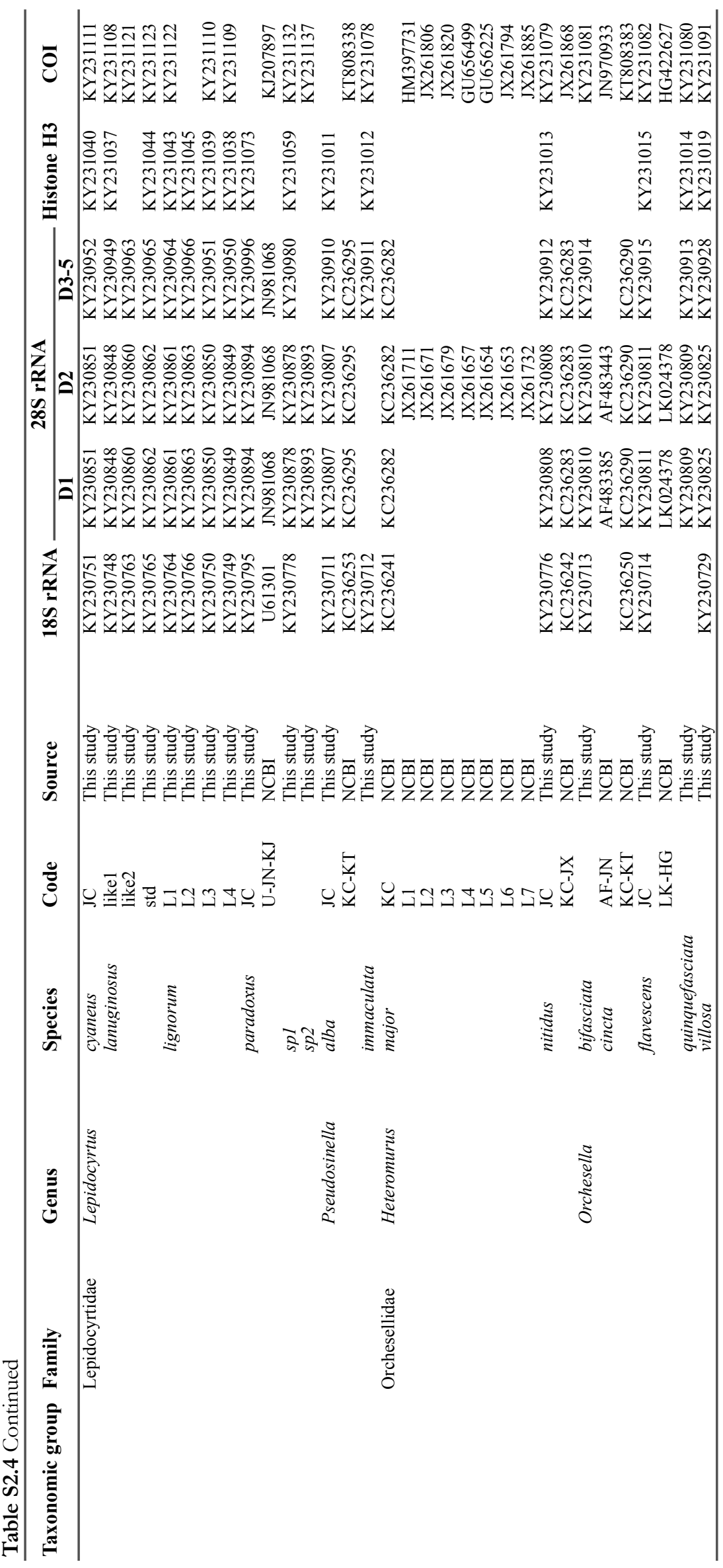




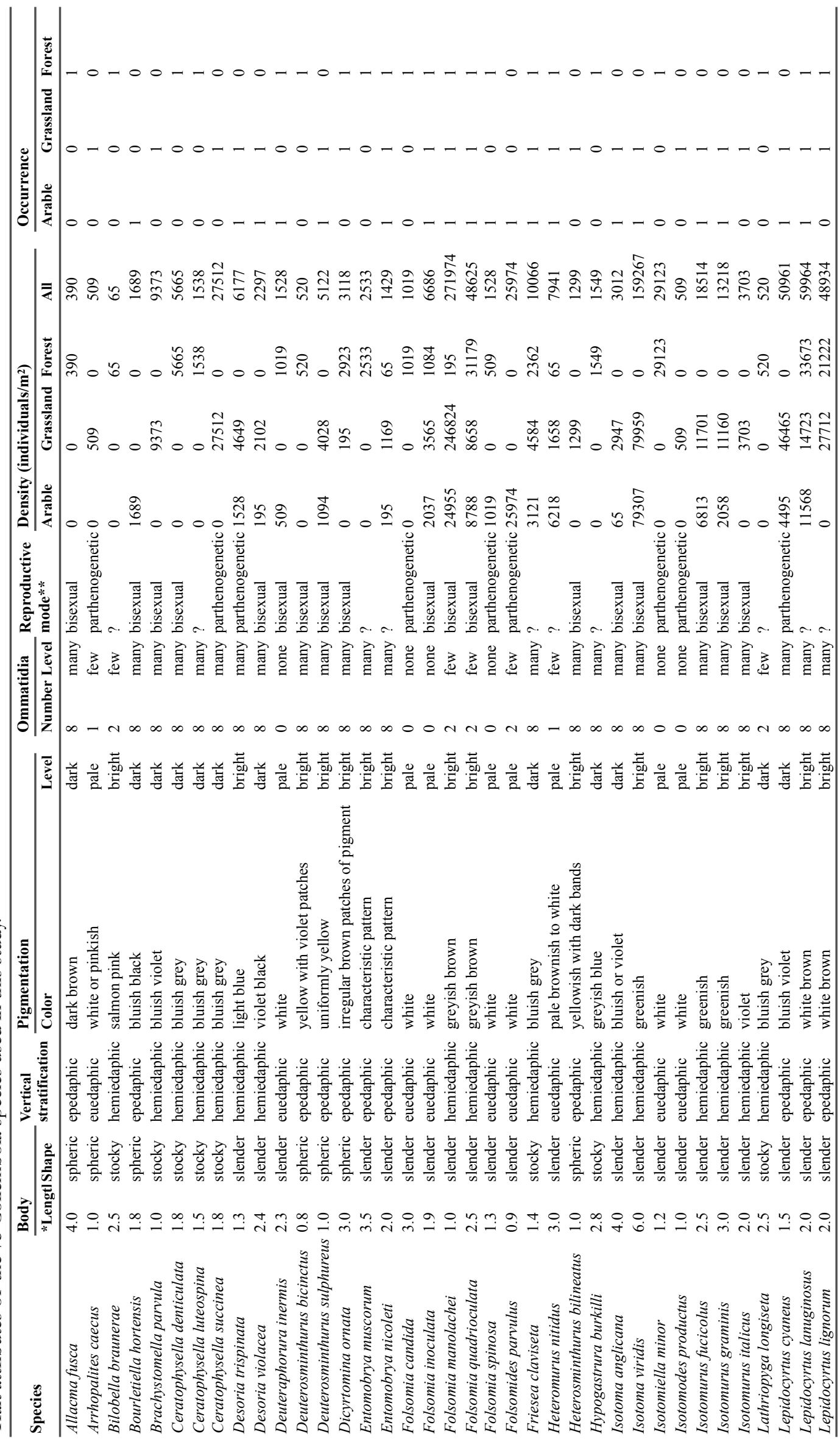




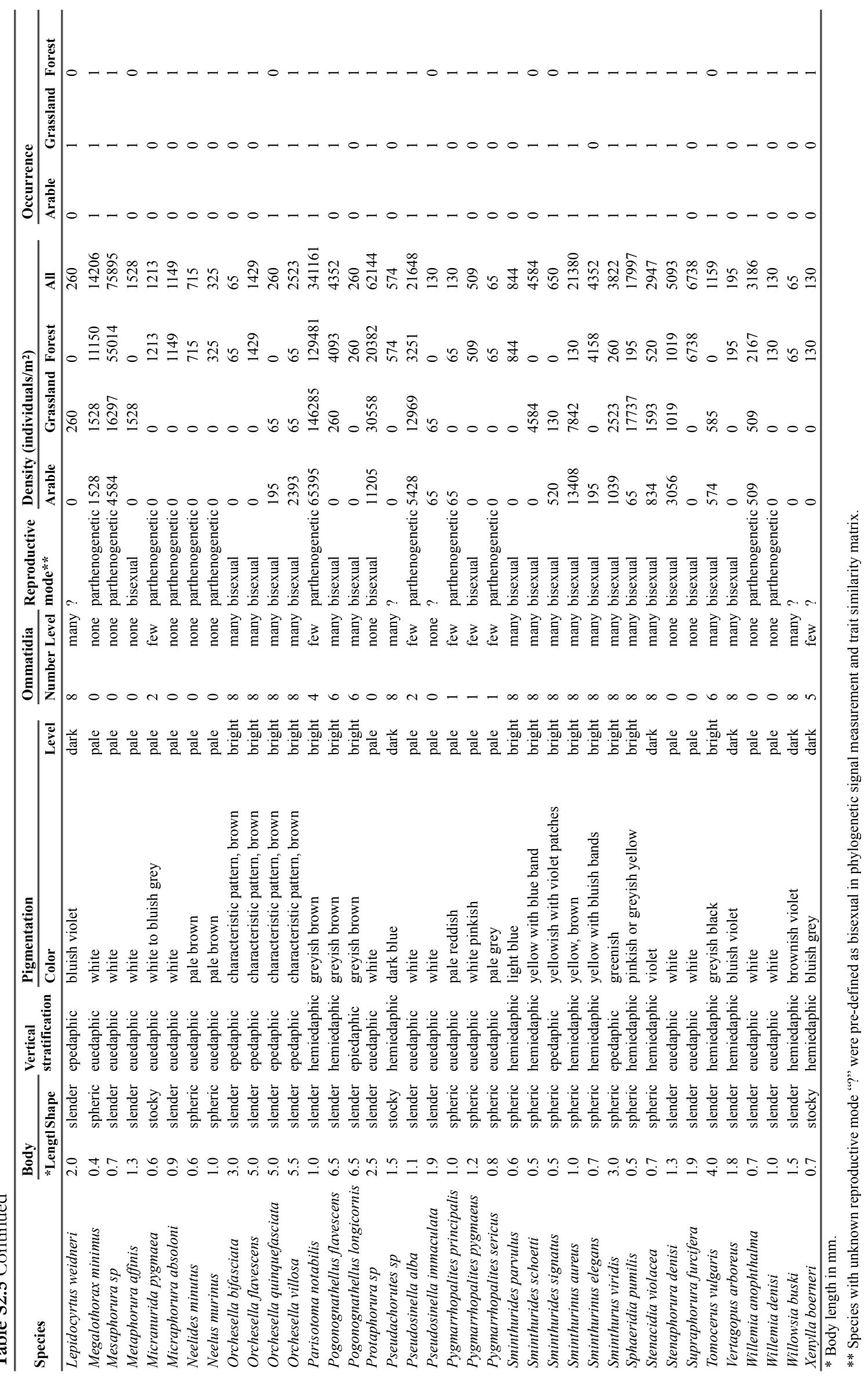


Table S2.6(a)

Estimated number of transitions across 1,000 stochastic character mapping simulations of body shape of Collembola $(\mathrm{N}=6$, median; $5.8 \pm 1.0$, mean $\pm \mathrm{SD})$.

\begin{tabular}{|c|c|c|c|c|c|c|c|c|c|c|c|c|}
\hline \multirow{3}{*}{$\begin{array}{l}\text { From: } \\
\text { Slender }\end{array}$} & \multicolumn{4}{|c|}{ Slender } & \multicolumn{4}{|c|}{ Stocky } & \multicolumn{4}{|c|}{ Spheric } \\
\hline & Median & Mean & \pm & SD & Median & Mean & \pm & SD & Median & Mean & \pm & SD \\
\hline & & & & & 1 & 1.2 & \pm & 0.7 & 2 & 2.3 & \pm & 0.6 \\
\hline Stocky & 2 & 1.9 & \pm & 0.7 & & & & & 0 & 0.1 & \pm & 0.4 \\
\hline Spheric & $\mathbf{0}$ & 0.2 & \pm & 0.7 & $\mathbf{0}$ & 0.1 & \pm & 0.3 & & & & \\
\hline
\end{tabular}

Table S2.6(b)

Estimated number of transitions across 1,000 stochastic character mapping simulations of vertical stratification of Collembola $(\mathrm{N}=25$, median; $26.1 \pm 4.8$, mean $\pm \mathrm{SD})$.

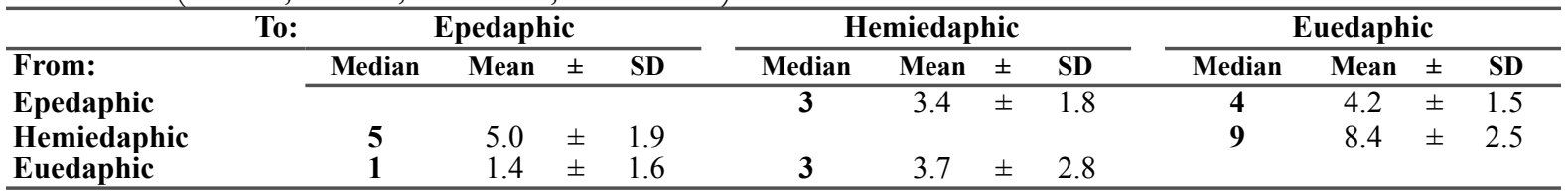

Table S2.6(c)

Estimated number of transitions across 1,000 stochastic character mapping simulations of pigmentation of Collembola $(\mathrm{N}=33$, median; $34.3 \pm 7.1$, mean $\pm \mathrm{SD})$.

\begin{tabular}{|c|c|c|c|c|c|c|c|c|c|c|c|c|}
\hline & To: & Dark & & & & Bright & & & & Pale & & \\
\hline From: & Median & Mean & \pm & $\overline{\text { SD }}$ & Median & Mean & \pm & $\begin{array}{l}\text { SD } \\
\end{array}$ & Median & Mean & \pm & SD \\
\hline Dark & & & & & 4 & 4.4 & \pm & 2.6 & 5 & 5.1 & \pm & 2.3 \\
\hline Bright & 8 & 8.6 & \pm & 2.4 & & & & & 8 & 7.9 & \pm & 2.9 \\
\hline Pale & 3 & 3.3 & \pm & 2.5 & 4 & 5.0 & \pm & 3.1 & & & & \\
\hline
\end{tabular}

Table S2.6(d)

Estimated number of transitions across 1,000 stochastic character mapping simulations of number of ommatidia of Collembola $(\mathrm{N}=19$, median; $19.8 \pm 3.3$, mean $\pm \mathrm{SD})$.

\begin{tabular}{|c|c|c|c|c|c|c|c|c|c|c|c|c|}
\hline \multirow{3}{*}{$\begin{array}{l}\text { From: } \\
\text { Many }\end{array}$} & To: & \multicolumn{3}{|l|}{ Many } & \multicolumn{4}{|c|}{ Few } & \multicolumn{4}{|c|}{ None } \\
\hline & Median & Mean & \pm & SD & Median & Mean & \pm & SD & Median & Mean & \pm & SD \\
\hline & & & & & 6 & 6.5 & \pm & 1.4 & 8 & 7.7 & \pm & 1.6 \\
\hline Few & 1 & 0.8 & \pm & 1.0 & & & & & 1 & 1.0 & \pm & 1.2 \\
\hline None & 1 & 1.3 & \pm & 1.4 & 3 & 2.7 & \pm & 1.1 & & & & \\
\hline
\end{tabular}

Table S2.6(e)

Estimated number of transitions across 1,000 stochastic character mapping simulations of reproductive mode of Collembola $(\mathrm{N}=19$, median; $19.9 \pm 4.26$, mean $\pm \mathrm{SD})$.

\begin{tabular}{|c|c|c|c|c|c|c|c|c|c|}
\hline \multirow{3}{*}{$\begin{array}{l}\text { From: } \\
\text { Parthenogenetic } \\
\text { Sexh }\end{array}$} & \multirow{2}{*}{ To: } & \multicolumn{4}{|c|}{ Parthenogenetic } & \multicolumn{4}{|c|}{ Sexual } \\
\hline & & Median & Mean & \pm & SD & Median & Mean & \pm & SD \\
\hline & & 13 & 13.9 & \pm & 2.5 & 5 & 6.0 & \pm & 2.8 \\
\hline
\end{tabular}




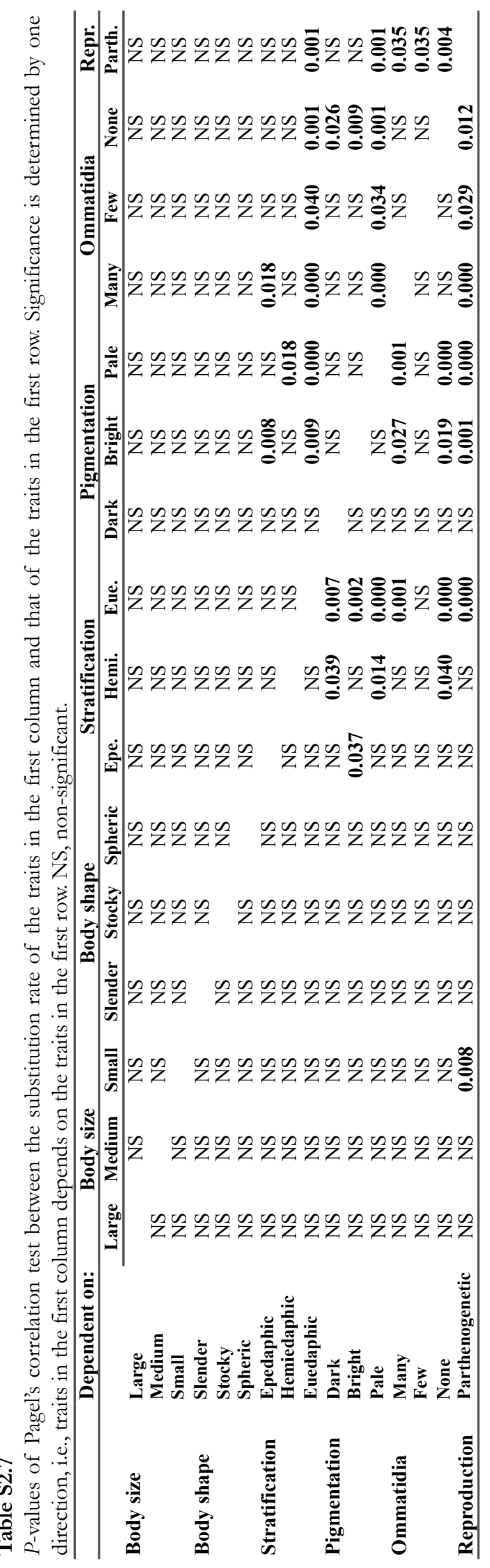


(a) BI tree

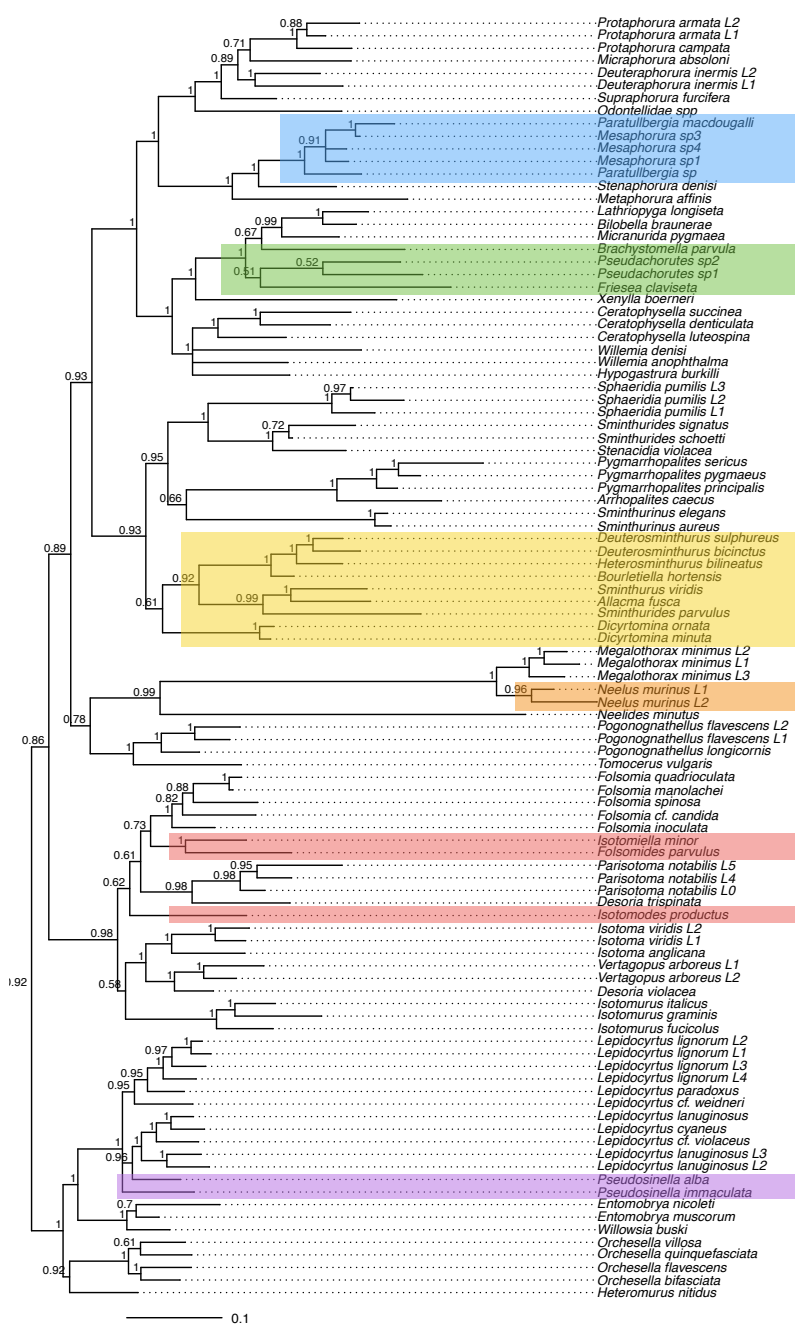

(b) ML tree

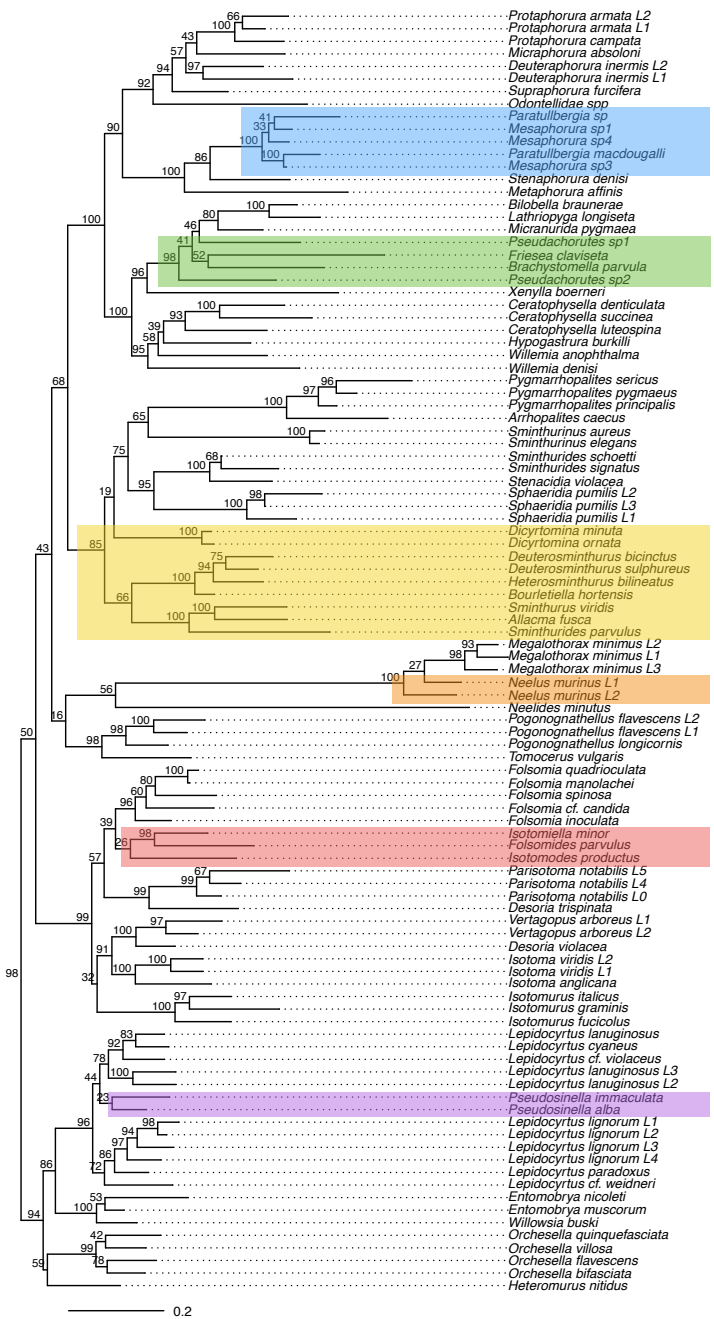

Figure S2.1

Comparison between Bayesian Inference (BI) tree and Maximum Likelihood (ML) tree based on 102 Collembola species and lineages. Coloring indicates inconsistency of phylogenetic relationships of taxa between BI and ML trees. 

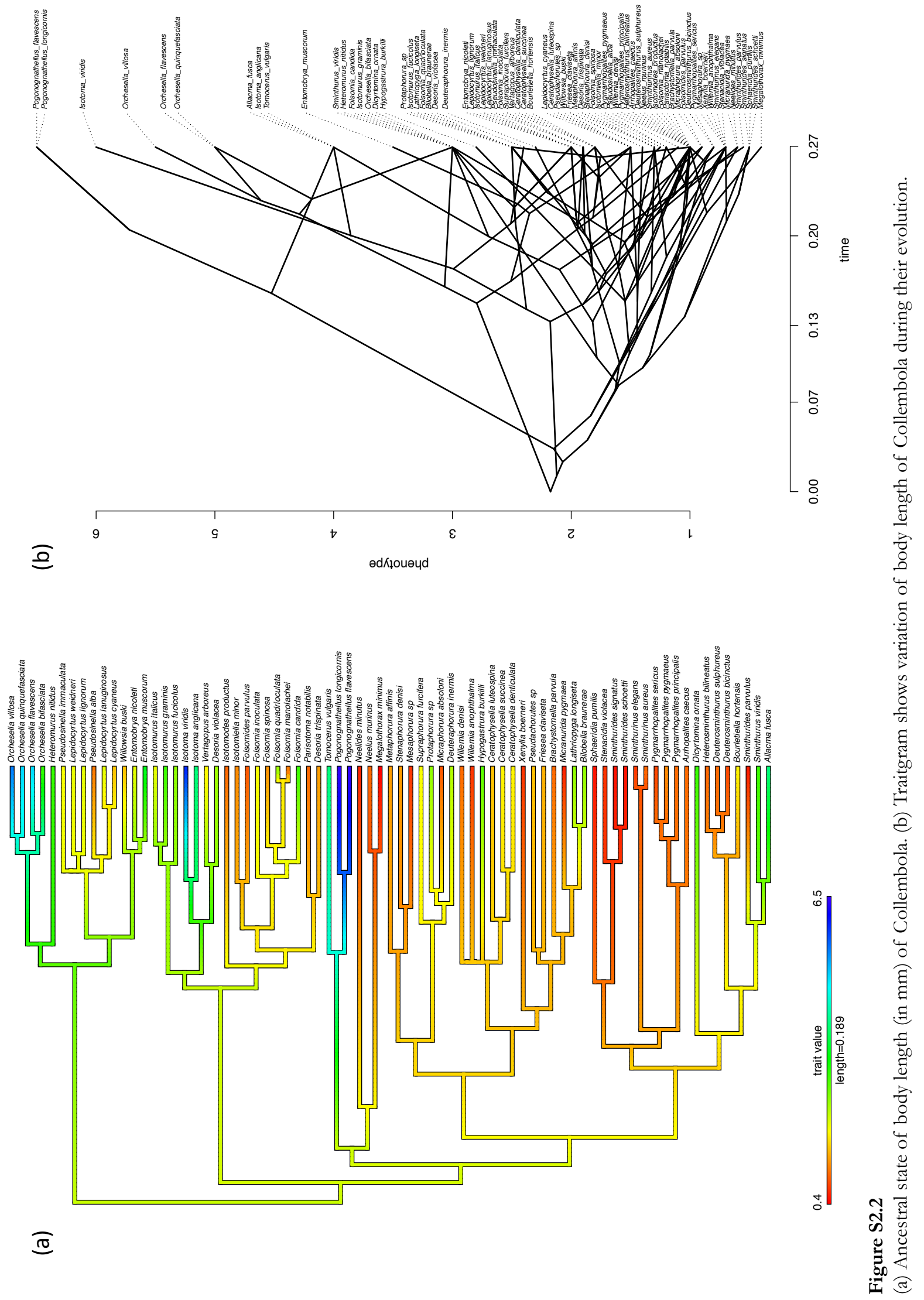

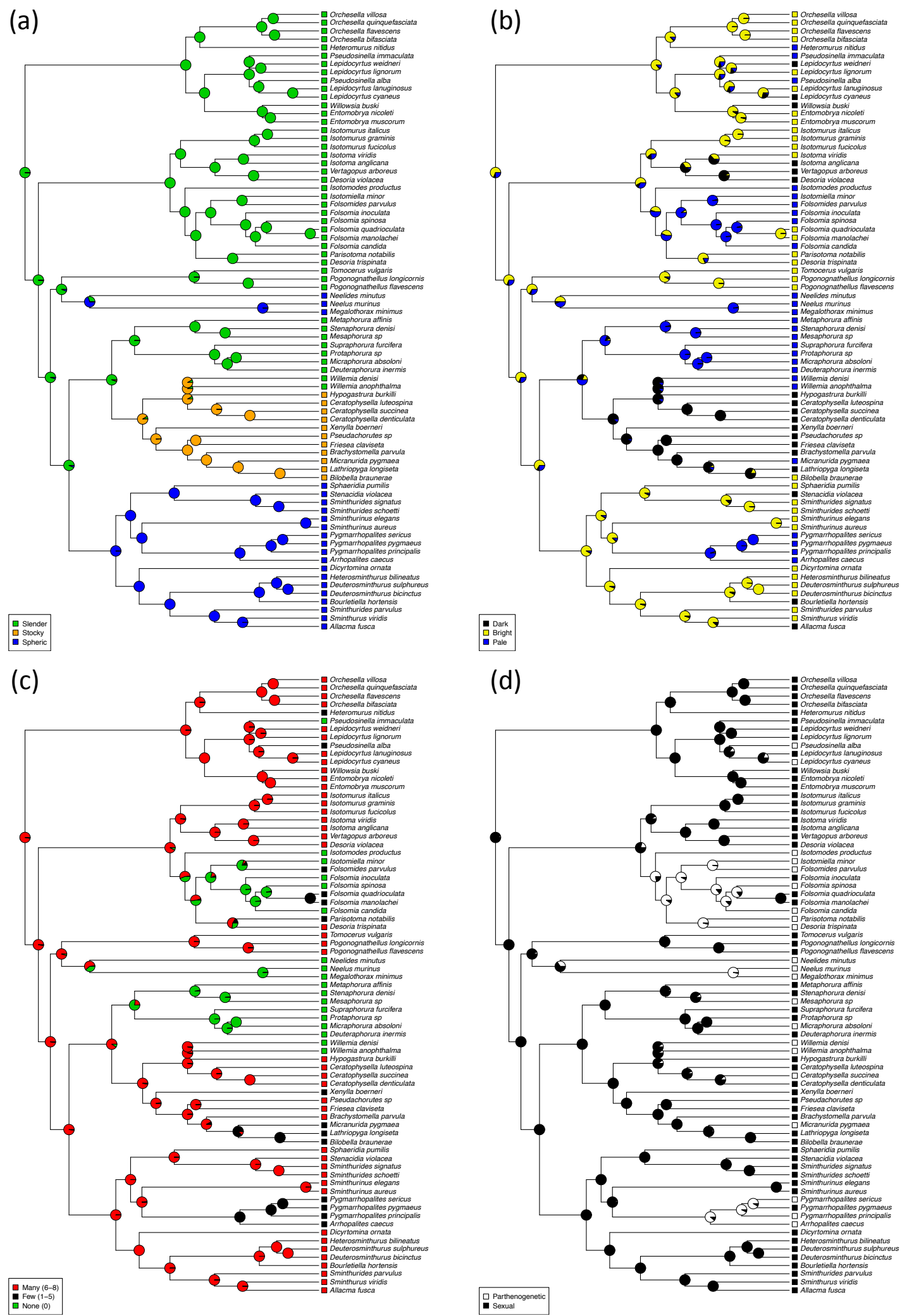

Figure S2.3

Ancestral state reconstruction of (a) body shape, (b) pigmentation, (c) number of ommatidia and (d) reproductive mode of Collembola. Colored squares at the tips of each branch represent the trait state of extant species. Pie charts on each node indicate the proportion of each character state summed across the posterior distribution of simulations. 


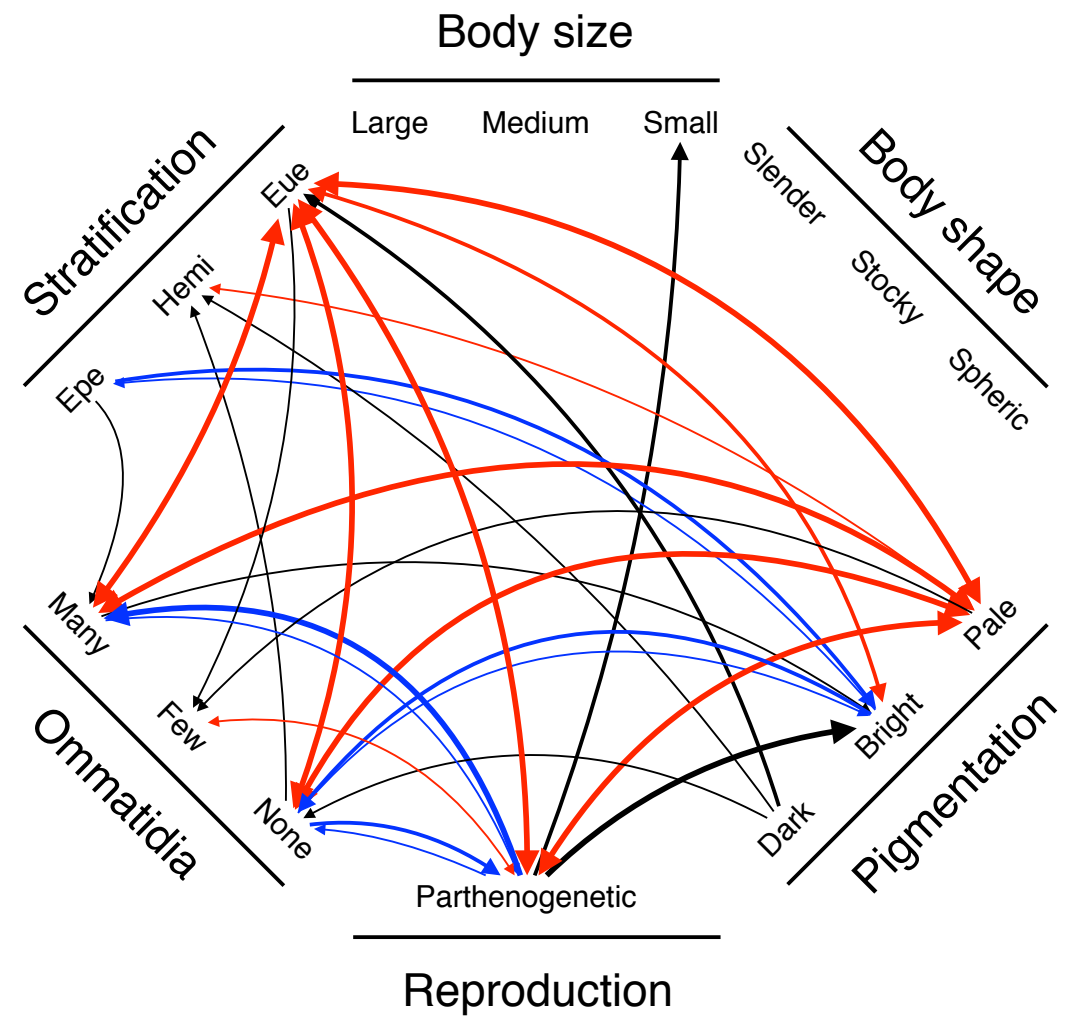

Figure S2.4

Results of Pagel's correlation test between the substitution rate of Collembola traits; significant dependence between two traits (i.e., " $\mathrm{x}$ " or " $\mathrm{y}$ " model) are indicated by arrows and lines with thickness reflecting the $P$-values. Red lines indicate that both directions (" $x$ " to " $y$ " and " $y$ " to " $x$ ") are significant with the same level of $P$-values $(<0.001,0.001-0.01$, or $0.01-0.05)$. Blue lines indicate that " $\mathrm{x}$ " and " $\mathrm{y}$ " as dependent variables give different $P$ values. Black lines indicate that only one direction is significance. 


\title{
Chapter 3
}

Published in Ecology and Evolution (2017) 7, 9624-9638.

\section{Neutral lipid fatty acid composition as trait and constraint in Collembola evolution}

Ting-Wen Chen, Philipp Sandmann, Ina Schaefer, Stefan Scheu

\begin{abstract}
Functional traits determine the occurrence of species along environmental gradients and their coexistence with other species. Understanding how traits evolved among coexisting species helps to infer community assembly processes. We propose fatty acid composition in consumer tissue as a functional trait related to both food resources and physiological functions of species. We measured phylogenetic signal in fatty acid profiles of 13 field-sampled Collembola (springtail) species and then combined the data with published fatty acid profiles of another 24 species. Collembola fatty acid profiles generally showed phylogenetic signal, with related species resembling each other. Long-chain polyunsaturated fatty acids, related to physiological functions, demonstrated phylogenetic signal. In contrast, most food resource biomarker fatty acids and the ratios between bacterial, fungal and plant biomarker fatty acids exhibited no phylogenetic signal. Presumably, fatty acids related to physiological functions have been constrained during Collembola evolutionary history: species with close phylogenetic affinity experienced similar environments during divergence, while niche partitioning in food resources among closely related species favored species coexistence. Measuring phylogenetic signal in ecologically relevant traits of coexisting species provides an evolutionary perspective to contemporary assembly processes of ecological communities. Integrating phylogenetic comparative methods with community phylogenetic and traitbased approaches may compensate for the limitations of each method when used alone and improve understanding of processes driving and maintaining assembly patterns.
\end{abstract}

\section{Keywords}

community phylogenetics; comparative method; functional traits; phylogenetic signal; springtails; trophic niche 


\section{Introduction}

Functional traits are measurable properties of species which influence their performance and fitness (Violle et al. 2007, Pey et al. 2014). They in part regulate the occurrence of species along environmental gradients and coexistence with other species in local communities (McGill et al. 2006, Ackerly and Cornwell 2007, Adler et al. 2013), where coexisting species may possess similar or different traits. Ecological traits have been assigned to two categories, $\alpha$ and $\beta$ niche traits. While $\beta$ niche traits determine species' environmental tolerance, $\alpha$ niche traits relate to resource exploitation (Ackerly and Cornwell 2007). Similar $\beta$ niche traits but different $\alpha$ niche traits thus allow species to live under similar environmental conditions but utilize different resources (Silvertown et al. 2006).

Understanding evolution of traits in coexisting species helps to infer community assembly processes (Webb et al. 2002, Silvertown et al. 2006, Best and Stachowicz 2013). Species' traits may exhibit phylogenetic signal, i.e., phylogenetically related species share similar traits derived from a common ancestor (Harvey and Pagel 1991). In contrast, traits may evolve convergently, resulting in closely related species with dissimilar traits or distantly related species with similar traits (Cavender-Bares et al. 2004). However, species' traits may also be labile, i.e., varying among species irrespective of phylogenetic relationships. Further, $\alpha$ and $\beta$ niche traits may evolve in different ways and thus exhibit different phylogenetic signal: $\beta$ niche traits are usually phylogenetically conserved, while $\alpha$ niche traits tend to be evolutionarily labile (Silvertown et al. 2006, Ackerly et al. 2006, Best and Stachowicz 2013). In this study, we measured phylogenetic signal in a ubiquitous trait of terrestrial microarthropods, i.e., fatty acid composition.

Fatty acids (FAs) are major components of lipids, serving as a source of energy (i.e., neutral lipids) and structural components of cell membranes (i.e., phospholipids; Ruess and Chamberlain 2010). Neutral lipid fatty acids (NLFAs) in animal fat deposits carry the signal of the diet. Some NLFAs are incorporated directly and unmodified from food resources and are useful as biomarkers to distinguish between major food resources in animals living in soil (Ruess and Chamberlain 2010, Buse et al. 2013, Ferlian et al. 2015). These biomarker FAs include absolute bacterial biomarkers which are only synthesized by prokaryotes,

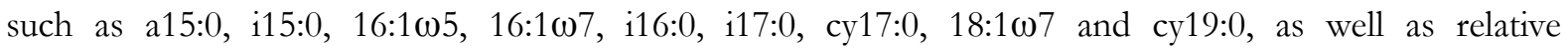

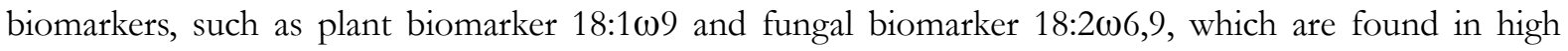
proportions when the consumer mainly feeds on plant or fungi, respectively. Thus, proportions of biomarker FAs imply $\alpha$ niche traits related to food resources. Other NLFAs, such as C20 polyunsaturated

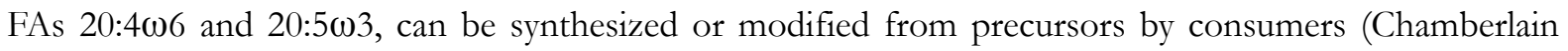
and Black 2005, Ruess and Chamberlain 2010). These FAs are essential for biosynthesis of other compounds such as prostaglandins and eicosanoids, which are associated with reproduction, immune response and temperature regulation (Chamberlain et al. 2004, Chamberlain and Black 2005, Haubert et al. 2008). They thus represent $\beta$ niche traits reflecting species environmental requirements. 
Springtails (Hexapoda: Collembola) are among the most abundant soil invertebrates. They occur in virtually every terrestrial habitat reaching particularly high densities in soil and contribute to decomposition processes and nutrient cycling in terrestrial ecosystems (Rusek 1998). They are ideal for exploring phylogenetic signal of FAs as they consume a wide range of food resources including detritus, roots and root exudates, bacteria, fungi and algae (Hopkin 1997). Fatty acid profiles have been used to identify food resources of Collembola and their association with different decomposition channels based on bacteria, fungi or root exudates as basal resources (Ruess et al. 2005, Pollierer et al. 2012, Ferlian et al. 2015). Distinct FA profiles of different Collembola species suggest trophic niche differentiation among co-occurring species (Chamberlain and Black 2005, Ruess et al. 2007, Ferlian et al. 2015). This may be attributed to (1) taxonomic or evolutionary relationships between different phylogenetic groups (Chamberlain and Black 2005), reflecting fixation of the physiology of species and their way of feeding over evolutionary time, and/or (2) ecological characteristics, such as life-forms (eu-, hemi-, and epedaphic) or availability of food resources in a habitat (Ruess et al. 2007). Further, species assigned to different soil strata may have similar FA profiles, indicating the use of similar resources (Ferlian et al. 2015). Overall, FA composition of Collembola may be similar in closely related species (phylogenetic signal present) and/or determined by available resources and thus not related to phylogenetic affinity (phylogenetic signal absent).

In this study we consider FA composition as a functional trait and analyze its phylogenetic signal using a comparative method (Harvey and Pagel 1991, Freckleton et al. 2002). Based on the $\alpha$ and $\beta$ niche trait concept, we tested the following hypotheses: (1) C20 polyunsaturated FAs exhibit phylogenetic signal in Collembola, suggesting that closely related species have similar physiological attributes. (2) Food resource FA biomarkers in Collembola are phylogenetically independent as different species utilize different resources. We used two FA datasets: FA profiles measured in this study from 13 field-sampled Collembola species and our data combined with published FA profiles of another 24 species (Table 3.1). We constructed a phylogenetic tree for all 37 Collembola species and measured phylogenetic signal in both FA datasets using two common comparative phylogenetic metrics, Blomberg's K (Blomberg et al. 2003) and Pagel's lambda (Pagel 1999, Freckleton et al. 2002).

\section{Materials and Methods}

\section{Sampling}

Collembola were sampled from two sites near Göttingen, Germany, Deppoldshausen $\left(51.575^{\circ} \mathrm{N}\right.$, $\left.9.973^{\circ} \mathrm{E}\right)$ and Ossenfeld $\left(51.548^{\circ} \mathrm{N}, 9.798^{\circ} \mathrm{E}\right)$. Each sampling site was composed of three adjacent habitats: arable field, pasture and forest. In each habitat, five samples $\left(1 \mathrm{~m}^{2}\right.$, at least $5 \mathrm{~m}$ apart) were taken in June and July 2015. Collembola in arable fields and pastures were sampled using an aspirator then immediately brought to the laboratory at the University of Göttingen and sorted. Collembola in forests were extracted from leaf litter by heat (Kempson et al. 1963) at constant $35^{\circ} \mathrm{C}$ for one week. Collembola were sampled 


\section{Table 3.1}

Taxonomy and collection habitat of the Collembola species used in this study. The 13 species collected for this study are marked in bold.

\begin{tabular}{|c|c|c|c|c|}
\hline Phylogenetic group & Family & Species* & Habitat** & Reference \\
\hline \multirow[t]{11}{*}{ Symphypleona } & \multirow[t]{5}{*}{ Sminthuridae } & \multirow[t]{3}{*}{ Allacma fusca } & \multicolumn{2}{|c|}{ Arable field (1) This study } \\
\hline & & & Forest (3) & This study \\
\hline & & & Forest & Chamberlain and Black (2005) \\
\hline & & \multirow[t]{2}{*}{ Sminthurus viridis } & Arable field (1) & This study \\
\hline & & & Grassland (5) & This study \\
\hline & \multirow[t]{2}{*}{ Bouletiellidae } & \multirow[t]{2}{*}{ Deuterosminthurus sulphureus } & Arable field (1) & This study \\
\hline & & & Grassland (2) & This study \\
\hline & \multirow[t]{4}{*}{ Dicyrtomidae } & \multirow[t]{3}{*}{ Dicyrtomina ornata } & Forest & Chamberlain and Black (2005) \\
\hline & & & Forest & Ruess et al. (2005) \\
\hline & & & Forest & Ruess et al. (2007) \\
\hline & & Dicyrtomina sp. (D. saundersi) & Forest & Ruess et al. (2005) \\
\hline \multirow[t]{15}{*}{ Poduromorpha } & \multirow[t]{7}{*}{ Hypogastruridae } & \multirow[t]{4}{*}{ Ceratophysella denticulata } & Forest (3) & This study \\
\hline & & & Forest & Ruess et al. (2005) \\
\hline & & & Forest & Ruess et al. (2007) \\
\hline & & & Forest & Ferlian et al. (2015) \\
\hline & & Ceratophysella succineal & Grassland & Sechi et al. (2014) \\
\hline & & \multirow[t]{2}{*}{ Willemia anophthalma } & Arable field & Ngosong et al. (2009) \\
\hline & & & Arable field & Ngosong et al. (2011) \\
\hline & Brachystomellidae & Brachystomella parvula ${ }^{1}$ & Grassland & Sechi et al. (2014) \\
\hline & \multirow[t]{3}{*}{ Neanuridae } & \multirow[t]{2}{*}{ Neanura muscorum } & Forest & Ruess et al. (2005) \\
\hline & & & Forest & Ruess et al. (2007) \\
\hline & & Polyacanthella (Friesea claviseta) & Arable field & Ngosong et al. (2009) \\
\hline & \multirow[t]{4}{*}{ Onychiuridae } & Onychiurus spp. (O. ambulans) & Forest & Ruess et al. (2005) \\
\hline & & Protaphorura armata & Forest & Ferlian et al. (2015) \\
\hline & & Protaphorura fimata (P. sp1) & Arable field & Haubert et al. (2009) \\
\hline & & Protaphorura spp. (P. sp2) & Forest & Ruess et al. (2007) \\
\hline \multirow[t]{8}{*}{ Tomoceridae } & \multirow[t]{8}{*}{ Tomoceridae } & \multirow[t]{2}{*}{ Pogonognathellus flavescens } & Grassland (1) & This study \\
\hline & & & Forest (6) & This study \\
\hline & & \multirow[t]{3}{*}{ Pogonognathellus longicornis } & Forest & Chamberlain and Black (2005) \\
\hline & & & Forest & Ruess et al. (2005) \\
\hline & & & Forest & Ruess et al. (2007) \\
\hline & & Tomocerus vulgaris & Forest (4) & This study \\
\hline & & Tomocerus baudoti & Forest & Pollierer et al. (2012) \\
\hline & & Tomocerus minor & Forest & Chamberlain and Black (2005) \\
\hline \multirow[t]{16}{*}{ Isotomidae } & Isotomidae & Isotoma viridis & Arable field (4) & This study \\
\hline & & & Grassland (4) & This study \\
\hline & & & Arable field & Ngosong et al. (2009) \\
\hline & & & Arable field & Ngosong et al. (2011) \\
\hline & & & Forest & Chamberlain and Black (2005) \\
\hline & & Isotoma viridis $^{2}$ & Grassland & Sechi et al. (2014) \\
\hline & & Isotoma anglicana ${ }^{2}$ & Grassland & Sechi et al. (2014) \\
\hline & & Desoria violacea & Forest & Ruess et al. (2005) \\
\hline & & & Forest & Ruess et al. (2007) \\
\hline & & Folsomia quadrioculata & Forest & Ruess et al. (2005) \\
\hline & & & Forest & Ruess et al. (2007) \\
\hline & & & Forest & Ferlian et al. (2015) \\
\hline & & Isotomiella minor & Forest & Ferlian et al. (2015) \\
\hline & & Isotomurus palustris (I. fucicolus) & Forest & Chamberlain and Black (2005) \\
\hline & & Parisotoma notabilis & Forest & Ruess et al. (2005) \\
\hline & & & Forest & Ferlian et al. (2015) \\
\hline
\end{tabular}


Table 3.1 Continued

\begin{tabular}{|c|c|c|c|c|}
\hline Phylogenetic group & Family & Species* & Habitat $* *$ & Reference \\
\hline \multirow[t]{23}{*}{ Entomobryoidea } & Entomobryidae & Entomobrya muscorum & Grassland (2) & This study \\
\hline & & & Forest (5) & This study \\
\hline & & & Forest & Ruess et al. (2005) \\
\hline & & Entomobrya nicoleti & Grassland (2) & This study \\
\hline & & Entomobrya nivalis & Forest & Ruess et al. (2005) \\
\hline & Lepidocyrtidae & Pseudosinella immaculata & Grassland (1) & This study \\
\hline & & Lepidocyrtus cyaneus & Arable field (4) & This study \\
\hline & & & Grassland (5) & This study \\
\hline & & & Grassland & Sechi et al. (2014) \\
\hline & & Lepidocyrtus lanuginosus & Arable field (2) & This study \\
\hline & & & Grassland (4) & This study \\
\hline & & & Forest (1) & This study \\
\hline & & & Forest & Pollierer et al. (2012) \\
\hline & & & Forest & Ferlian et al. (2015) \\
\hline & & Lepidocyrtus lignorum & Forest & Ruess et al. (2005) \\
\hline & & & Forest & Ruess et al. (2007) \\
\hline & & Lepidocyrtus curvicollis (L. sp) & Forest & Chamberlain and Black (2005) \\
\hline & Orchesellidae & Orchesella villosa & Arable field (8) & This study \\
\hline & & & Forest (1) & This study \\
\hline & & & Forest & Chamberlain and Black (2005) \\
\hline & & & Arable field & Haubert et al. (2009) \\
\hline & & Orchesella flavescens & Forest & Ruess et al. (2005) \\
\hline & & & Forest & Ruess et al. (2007) \\
\hline
\end{tabular}

* Name in parenthesis indicates the congeneric species used in the phylogeny constructed by sequences listed in Table S3.1.

** Number in parenthesis indicates replicate number in fatty acid measurements of field derived Collembola in this study (pooled for sites)

${ }^{1}$ Fatty acid data compiled using Poduromorpha in Sechi et al. 2014

2 Fatty acid data compiled using Isotoma spp. in Sechi et al. 2014

alive daily and immediately stored at $-80^{\circ} \mathrm{C}$ until identification and lipid extraction. Species were identified according to Hopkin (2007). In total, sufficient biomass for FA extraction was obtained for 13 species.

\section{Fatty acid analysis}

Soil and organic matter was removed from the surface of each Collembola using a brush prior to FA extraction. Depending on body size of individuals and species, three to 36 individuals of the same species and sample were pooled for one FA extraction. In total, $70 \mathrm{FA}$ measurements were obtained, ranging from one to four replicates for each species per habitat and site.

NLFAs were extracted as described in Haubert et al. (2004). Neutral lipid fractions were dried at $50^{\circ} \mathrm{C}$ using a rotation vacuum concentrator (RVC 2-25, Chris, Osterode am Harz, Germany). The lipid fractions were then saponified, methylated and washed. The obtained FA methyl esters were transferred into vials, capped and stored at $-21^{\circ} \mathrm{C}$ until gas chromatography (GC) analysis. The gas chromatograph (Clarus 500, Perkin Elmer, Waltham, USA) was equipped with a flame ionization detector (PE-5 capillary column, $30 \mathrm{~m} \times 0.32 \mathrm{~mm}$ i.d., $0.25 \mathrm{~mm}$ film thickness, Perkin Elmer, Waltham, USA) and helium as carrier gas. The analysis program followed Ferlian and Scheu (2014). FA methyl esters were identified by 
comparing retention times of samples and standard mixtures comprising unbranched and branched FA methyl esters.

\section{Collembola phylogeny}

In addition to the above 13 Collembola species, published NLFA data were available for 24 additional species (Table 3.1). A phylogeny of all 37 Collembola species, spanning 12 families, was constructed using six genetic markers: $18 \mathrm{~S}$ rRNA, $28 \mathrm{~S}$ rRNA D1, D2 and D3 regions, cytochrome oxidase subunit I (COI) and Histone H3 genes. Callibaetis (Insecta: Ephemeroptera), Machilis (Insecta: Archaeognatha) and Zygentoma (Insecta: Zygentoma) were used as outgroups. Sequences were downloaded from GenBank (www.ncbi.nih.gov; Accession Number see Table S3.1). Species without sequence data available in GenBank were replaced by the taxonomically closest species, usually a congener (Table 3.1). The six genetic markers were aligned separately in R 3.2.2 (R Core Team 2015) using functions AlignSeqs and AdjustAlignment for $18 \mathrm{~S}$ and 28S rRNA and Histone H3 genes (package "DECIPHER"; Wright 2015) and function msaClustalW for COI by setting gap opening as 15 and gap extension as 6.6 (package "msa"; Bodenhofer et al. 2015). The aligned sequences were trimmed to the same length in BioEdit 7.2.5 (Hall 1999). Models of sequence evolution for each marker were obtained using jModelTest 2.1.4 and based on the Alkaike information criterion (Darriba et al. 2012). Terminal gaps in each marker set were replaced by "??" and the six markers were concatenated in a supermatrix (3,053 bp) using SequenceMatrix 1.8 (Vaidya et al. 2011). The phylogeny was inferred using Bayesian Inference (BI) in MrBayes 3.2.4 (Ronquist et al. 2012), setting models of sequence evolution for each marker separately as suggested by jModelTest. Bayesian Inference was conducted using two independent runs of four chains for 1,000,000 generations and the consensus tree generated using a burn-in of 0.25 . A second phylogenetic tree was constructed using Maximum Likelihood (ML) in RAxML 7.0.3 (Stamatakis 2006) based on the GTR+I+G model and 1,000 bootstrap replicates. The topologies of the phylogenetic trees of BI and ML were similar, except for the sister taxon of Poduromorpha. In the BI tree, Tomoceridae was sister of Poduromorpha (Figure S3.1), while in the ML tree it was Symphypleona (Figure S3.2). The BI tree was selected and transformed to an ultrametric tree using a penalized likelihood approach assuming different models of substitution rate variation among branches, including correlated, relaxed, discrete or strict clock models, using the function chronos implemented in the R package "ape" (Paradis et al. 2004). The ultrametric tree for downstream phylogenetic signal analyses was selected based on the smallest PHIIC value, a criterion analogous to Alkaike information criterion reflecting the best model fit to the data (Paradis 2013). Concomitantly, a strict clock model was used in the phylogenetic analyses. This tree was then used in the phylogenetic signal measurement. 


\section{Statistical analysis}

For our field data, rare FAs present in only single measurement and FAs contributing less than 1\% of total FAs were eliminated from the analyses. The remaining FAs were summed to $100 \%$ and the proportions of single FAs were logit-transformed using the function logit in the R package "car" (Fox and Weisberg 2011). To test for differences in FA compositions between Collembola species and habitats, multivariate analysis of variance (MANOVA) and discriminant function analysis (DFA, function lda implemented in the R package "MASS"; Venables and Ripley 2002) were used, with sites and habitats set as error terms in the model, followed by ANOVA with Holm's adjusted $P$-values (Holm 1979). For the FAs showing significant differences between Collembola species, Tukey's honestly significant difference (HSD) test was conducted. Fatty acid profiles of species were also explored using eigen decomposition principle components analysis (PCA). Species mean logit-transformed FA proportions were calculated and then multiplied by the eigenvectors based on a covariance matrix using the species mean. Individual observational logit-transformed FA proportions were multiplied by the same eigenvectors to examine intraspecific variation. Principle components (PCs) were selected if the variance explained by each axis was more than predicted by a broken stick model. Pearson correlation coefficients of FAs and PCs were calculated using function cor.test in $\mathrm{R}$ with Holm's $P$-value adjustment.

Three types of FA data were used to measure phylogenetic signal: (1) Species mean scores on the PC axes, irrespective of site and habitat; (2) species mean proportion of individual FAs; (3) species mean values of FA indices, including sums of bacterial FAs, plant-to-fungal FA marker ratios (P:F ratio), bacterial-to-fungal FA marker ratio (B:F ratio), bacterial-to-plant FA marker ratio (B:P ratio), Unsaturation Index (UI; Haubert et al. 2004), sums of saturated FAs (SFAs), monounsaturated FAs (MUFAs), polyunsaturated FAs (PUFAs) and C20 PUFAs and ratio of unsaturated to saturated FAs (U:S ratio). Phylogenetic signal was detected and quantified using both Blomberg's K (Blomberg et al. 2003) and Pagel's lambda (Pagel 1999, Freckleton et al. 2002). These two metrics assume a Brownian motion model of trait evolution, i.e., variance in trait values is directly proportional to branch length of a given phylogeny (Pagel 1999, Blomberg et al. 2003). Both methods were used because they have different sensitivities in detecting phylogenetic signal for traits evolved with various strengths of Brownian motion and for trees with different size (Münkemüller et al. 2012). Phylogenetic signal analyses were conducted using the function phylosig implemented in the R package "phytools" (Revell 2012). Standard errors of FA measurements were considered in Blomberg's K statistics (Ives et al. 2007). Significance tests were done by randomizing species on the phylogeny 10,000 times, to test whether trait values show phylogenetic signal or not (i.e., $\mathrm{HO}=0$ ). In case of significant $\mathrm{K}$-values of traits, the observed $\mathrm{K}$-value was further compared with 5,000 simulated $\mathrm{K}$-values to test whether phylogenetic signal was significantly different from the level expected under Brownian motion evolution model (i.e., $\mathrm{H} 0=1$; Revell et al. 2007). Simulations of trait values were conducted using the function fast BM in the R package "phytools" (Revell 2012). Lower and higher phylogenetic signal than predicted by a Brownian motion model was defined as a $\mathrm{K}$-value in the 0.025 and 0.975 quantiles of the log-transformed simulated $\mathrm{K}$-values, respectively. All $P$ - 
values in phylogenetic signal measurement were adjusted using Benjamini and Hochberg's method (Benjamini and Hochberg 1995). Phylogenetic signal of FAs was accepted only when both Blomberg’s K and Pagel's lambda were significant.

Since a small phylogenetic tree (13 species in our field-sampled dataset) may lack power to detect phylogenetic signal (Freckleton et al. 2002, Blomberg et al. 2003, Münkemüller et al. 2012), FA phylogenetic signal was also measured using a combined dataset comprising data of our field-sampled Collembola and published FA data (Table 3.1). Mean FA proportions were calculated for each species at each site and habitat for our FA data. Data from the literature were compiled at species level for each treatment or site by extracting the published mean values or recalculating original data provided by the authors. Due to inconsistency of FAs measured in different studies, only biomarker FAs, C20 unsaturated FAs and saturated FAs 16:0 and 18:0 were included. Unavailable values of these FAs in literature data were replaced by zero assuming that they were not reported due to being present in trace amounts only. Fatty acids contributing less than $1 \%$ of total FAs and those occurring in only one sample were eliminated. The remaining FAs were summed to $100 \%$ and logit-transformed, resulting in a final dataset of 37 species and 149 data points for phylogenetic signal measurements. Principle components and phylogenetic signal in species mean scores on PCA axes, mean proportion of individual FAs and FA indices were analyzed as above.

\section{Results}

\section{Fatty acid composition of Collembola}

Thirty-two FAs were identified from the 13 field-sampled Collembola species (Table S3.2). Frequent FAs (occurring in $>30$ of the 70 measurements) were 18:1 $199,18: 2 \omega 6,9,16: 0,18: 0,20: 5 \omega 3,20: 4 \omega 6$,

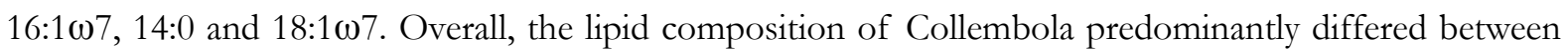
species, whereas the effect of habitat was not significant (MANOVA, $F_{384,288}=1.65, P<0.001$ for species and $F_{256,160}=1.26, P=0.058$ for habitat). The DFA plot clearly separated the FA profiles between different species (Figure 3.1). Allacma fusca, Deuterosminthurus sulphureus, Sminthurus viridis, Ceratophysella denticulata and Isotoma viridis were separated from the remaining species along the first two axes. The proportions of individual FAs differed among species (Table S3.2, S3.3). Fatty acid 18:169, a predominant FA in Collembola, was lower in I. viridis (12.4\%), while it contributed 26.5\%-42.2\% to total FAs in all other species. Another major FA, 18:2w6,9, was highest in the three Symphypleona species, $S$. viridis (37.6\%), A. fusca (34.9\%) and D. sulphureus (32.3\%). Fatty acid 16:0 was low in $A$. fusca (9.6\%), but high in all Entomobryoidea (23.3\%-26.4\%), except for Orchesella villosa (17.6\%). Fatty acid 18:0 was present in trace proportions in D. sulphureus (0.9\%), but was one of the main FAs in I. viridis (15.8\%). C20

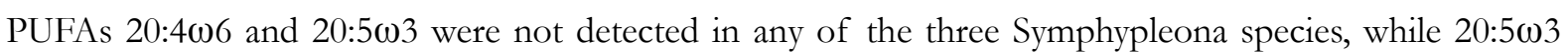
was high in the Tomoceridae, Pogonognathellus flavescens (8.1\%) and Tomocerus vulgaris (6.1\%). Fatty acid

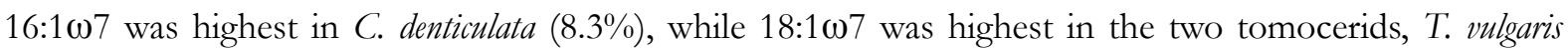


(6.7\%) and P. flavescens (5.7\%). Pseudosinella immaculata had a relatively high proportions of FA 14:0 $(11.4 \%)$.

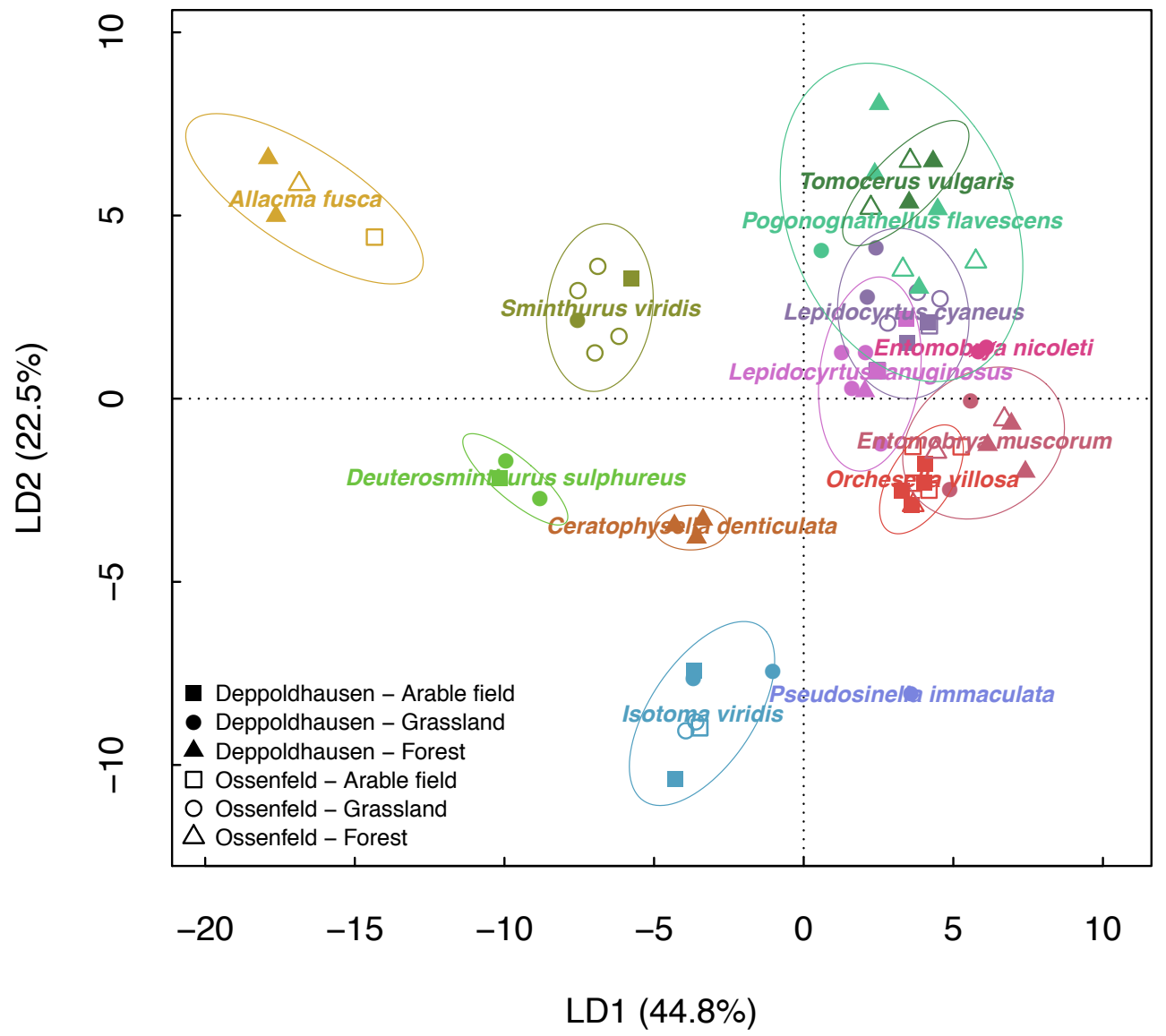

Figure 3.1

Discriminant function analysis of fatty acid profiles of 13 field-sampled Collembola species. Ellipses represent confidence ranges at $P=0.05$.

\section{Phylogenetic signal in FAs of sampled species}

The first four PCs together explained $84.2 \%$ of the variation in the FA profiles of the Collembola. PC1, representing $44.9 \%$ of the variation, showed phylogenetic signal consistent with predictions from the Brownian motion model, as indicated by both Blomberg's $\mathrm{K}$ and Pagel's lambda (Table 3.2). The PCA biplots indicated that three Symphypleona, A. fusca, D. sulphureus and S. viridis, had higher scores along PC1, which was negatively correlated with FA 18:0, 20:5

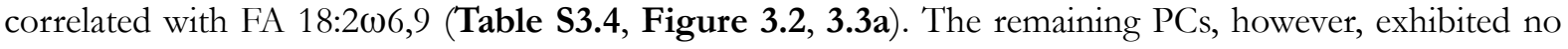
phylogenetic signal, except PC3 using Blomberg's K without $P$-value adjustment.

Proportions of the FAs 16:0, 18:1 $1 \omega 7,18: 2 \omega 6,9,20: 1 \omega 9$ and 20:5 $\omega 3$ showed significant phylogenetic signal as indicated by Blomberg's $\mathrm{K}$ after $P$-value adjustment. Pagel's lambda further indicated that the

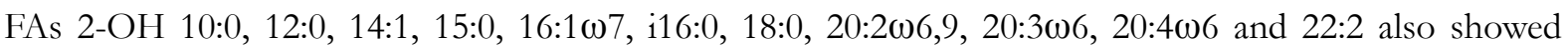


phylogenetic signal after P-value adjustment (Table 3.2). Phylogenetic signal in FA 16:0 resulted from higher proportions in the clade composed of Lepidocyrtidae and Entomobryidae and lower proportions in C. denticulata and Symphypleona. Phylogenetic signal in FA 20:1 09 resulted from the lack in the clades of Lepidocyrtidae (Lepidocyrtus and Pseudosinella), Entomobryidae (two Entomobrya species) and Sminthuridae (Allacma and Sminthurus). Notably, the K-value of 20:169 was larger than the 97.5\% quantile of simulated K-values, suggesting stronger phylogenetic signal than predicted by the Brownian motion model. Fatty acid 20:5 103 showed phylogenetic signal due to its consistently lower proportions in C. denticulata and Symphypleona, intermediate proportions in Entomobryoidea, higher proportions in Tomoceridae, and even higher proportions in I. viridis. Phylogenetic signal in the bacterial biomarker 18:1 107 reflected higher relative proportion in Tomoceridae and lower in Symphypleona and

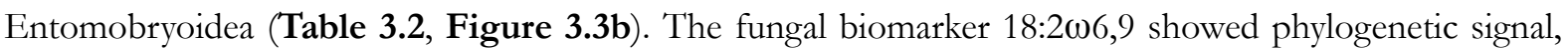
reflecting higher proportions in Symphypleona as well as lower proportions in most Entomobryoidea (Table 3.2, Figure 3.3c).

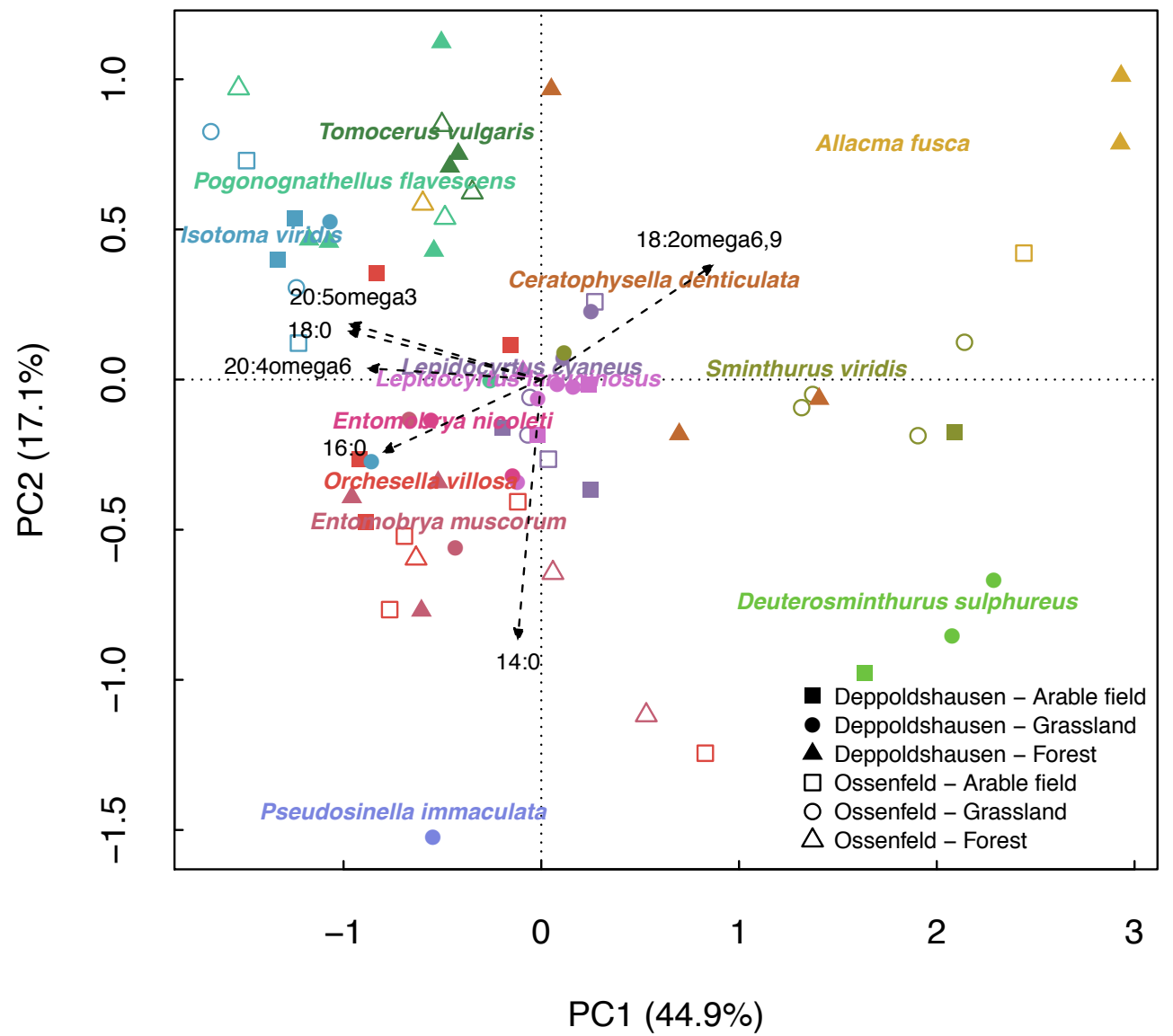

Figure 3.2

Biplots of principle components analysis using whole fatty acid profiles of 13 field-sampled Collembola species. Variation explained by each axis is given in parentheses. Position of species name represents its mean score on the axis irrespective of site and habitat. Only fatty acids significantly correlated to the PCs are plotted. 


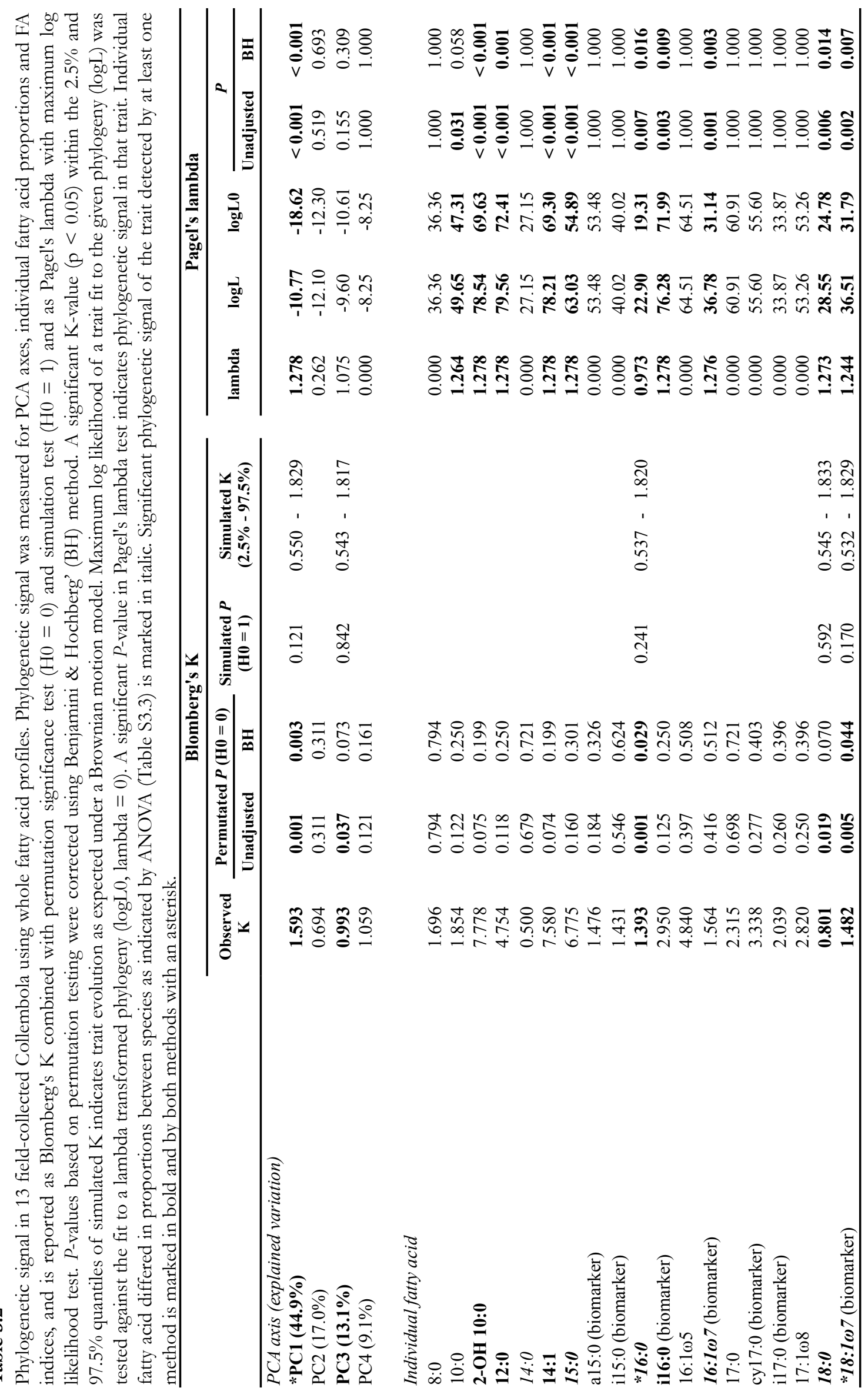




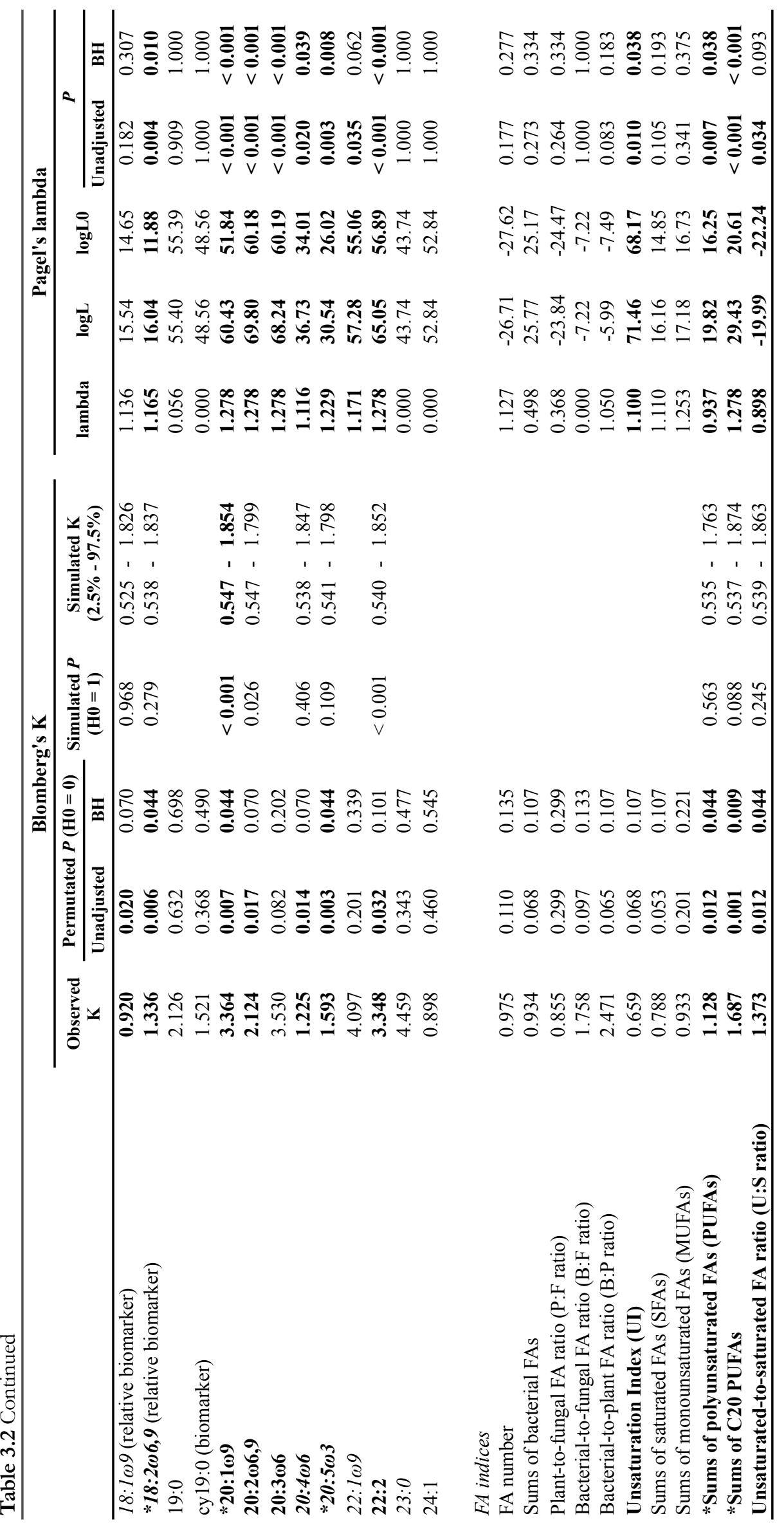




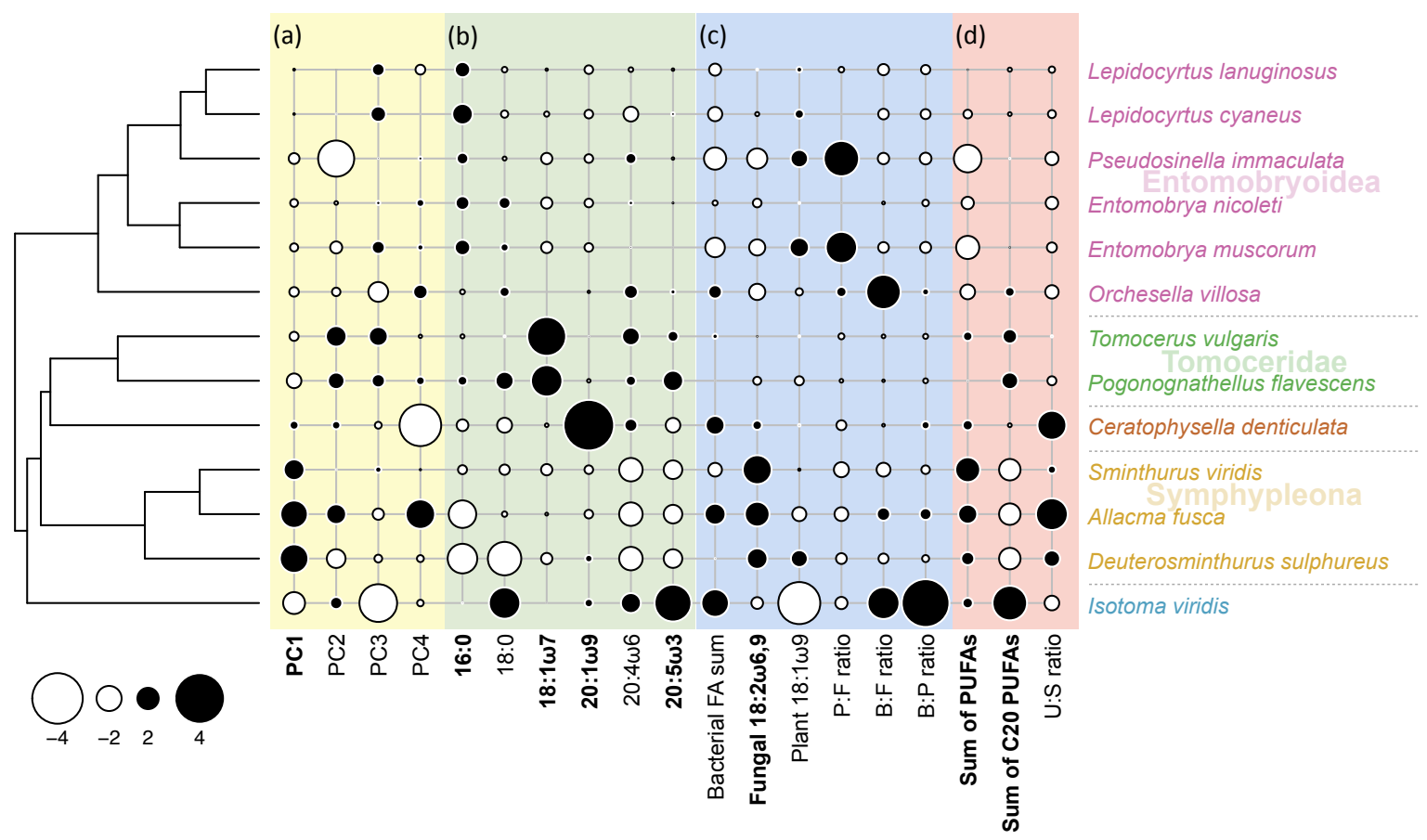

Figure 3.3

Relationship between phylogeny and selected trait values for field-sampled Collembola. Trait values were scaled and centralized before plotting. The size of the white and black circles indicates more negative or positive values, respectively. (a) Mean values of the scores of fatty acid profiles on the first four axes in principle components analysis (PCA), (b) proportions of individual fatty acids, (c) proportions of fatty acids derived from bacteria, fungi or plants, and the ratios between these three, and (d) summed proportions of polyunsaturated fatty acid and C20 polyunsaturated fatty acid, and ratio of unsaturated to saturated FAs; see Table 3.2 for abbreviations. Traits exhibiting phylogenetic signal as indicated by both Blomberg's K and Pagel's lambda are marked in bold.

The sum of C20 PUFAs and of all PUFAs exhibited phylogenetic signal according to both Blomberg's K and Pagel's lambda after P-value adjustment. The sum of C20 PUFAs was low in Symphypleona but high in I. viridis and the two Tomoceridae species (Table 3.2, Figure 3.3d). The sum of all PUFAs, however, was high in Symphypleona, C. denticulata and I. viridis but low in Entomobryoidea. The other FA indices, such as ratios between bacterial, fungal and plant biomarker fatty acids, showed no phylogenetic signal.

\section{Phylogenetic signal in FAs of combined dataset}

The first four PCs explained $76.4 \%$ of variation in the FA profiles of the 37 species of the combined dataset. PC1 explained 31.8\% of the variation in the FA profiles which was positively correlated with FA

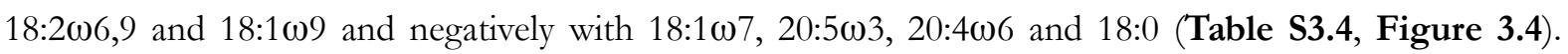
Phylogenetic signal in species mean scores at PC1 were driven by low scores in Tomoceridae and high scores in the clade of $A$. fusca, D. sulphureus and $S$. viridis (Table 3.3, Figure 3.5a). No phylogenetic signal was detected in species mean scores at the other three PCs. 
Analyses of phylogenetic signal in the mean proportions of individual FAs (Table S3.5) suggested that FA 18:0 and 20:5 133 exhibited phylogenetic signal as indicated by both Blomberg's $\mathrm{K}$ and Pagel's lambda (Table 3.3). Fatty acid 18:0 was high in the clade composed of Isotomurus palustris, Parisotoma notabilis, Isotomiella minor and Folsomia quadrioculata, while FA 20:5

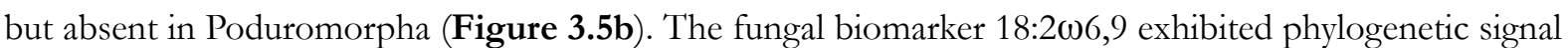
only by Pagel's lambda; however, plant biomarker 18:1 199 , the sum of bacterial FAs and the ratios between fungal, plant and bacterial FAs showed no phylogenetic signal (Table 3.3, Figure 3.5c). The sum of C20 PUFAs showed phylogenetic signal as indicated by both Blomberg's K and Pagel's lambda (Table 3.3). It was high in Tomocerus, intermediate in Entomobryoidea, and low in the clade of I. minor, F. quadrioculata and P. notabilis, as well as the clade of S. viridis, A. fusca and D. sulphureus (Figure 3.5d).

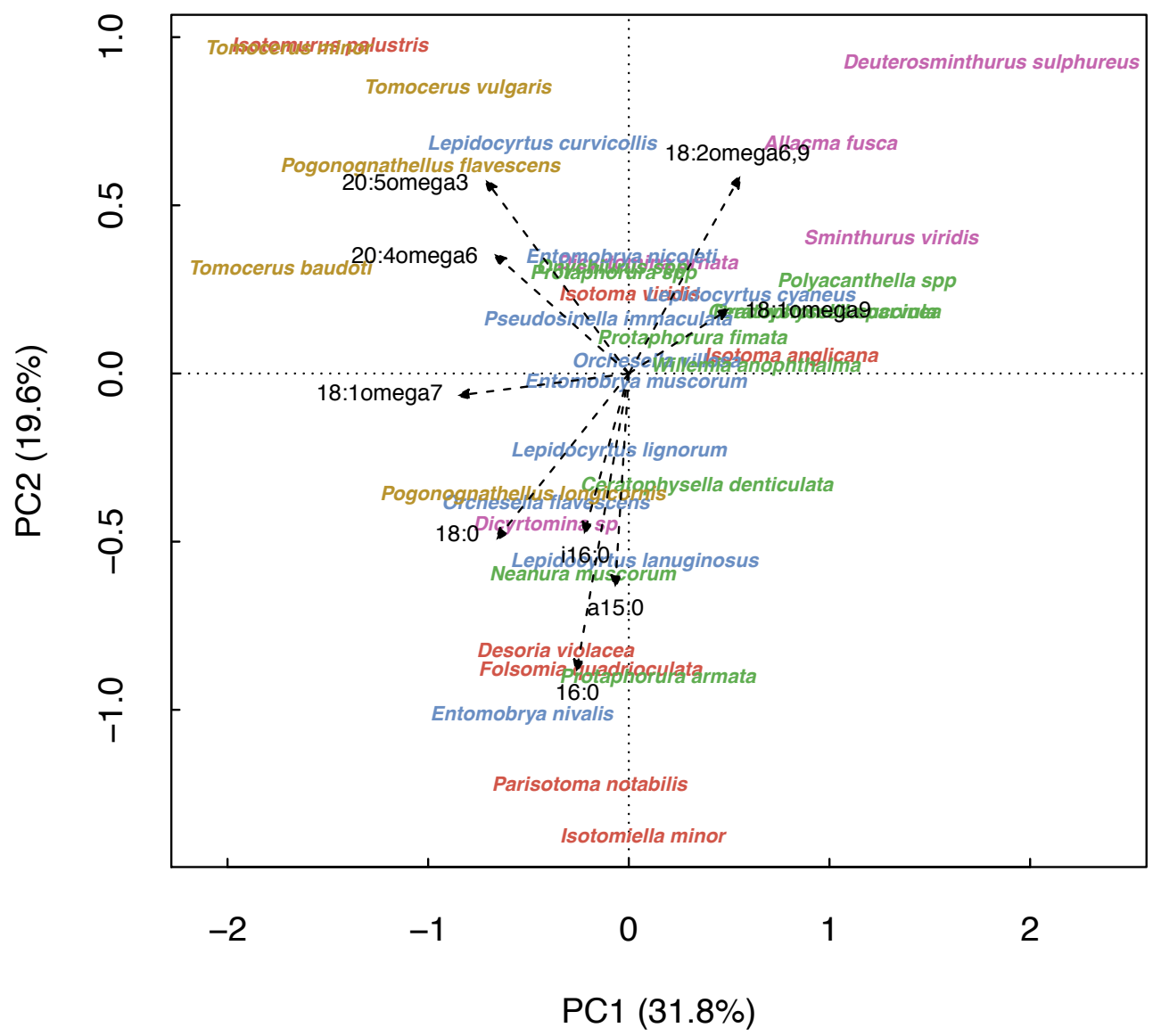

Figure 3.4

Principle components biplots of the variation in fatty acid profiles of the combined dataset. Variation explained by each axis is given in parentheses. Position of species name represents its mean score on the axis irrespective of reference, site, habitat and treatment; only fatty acids significantly correlated to the PCs are plotted. 


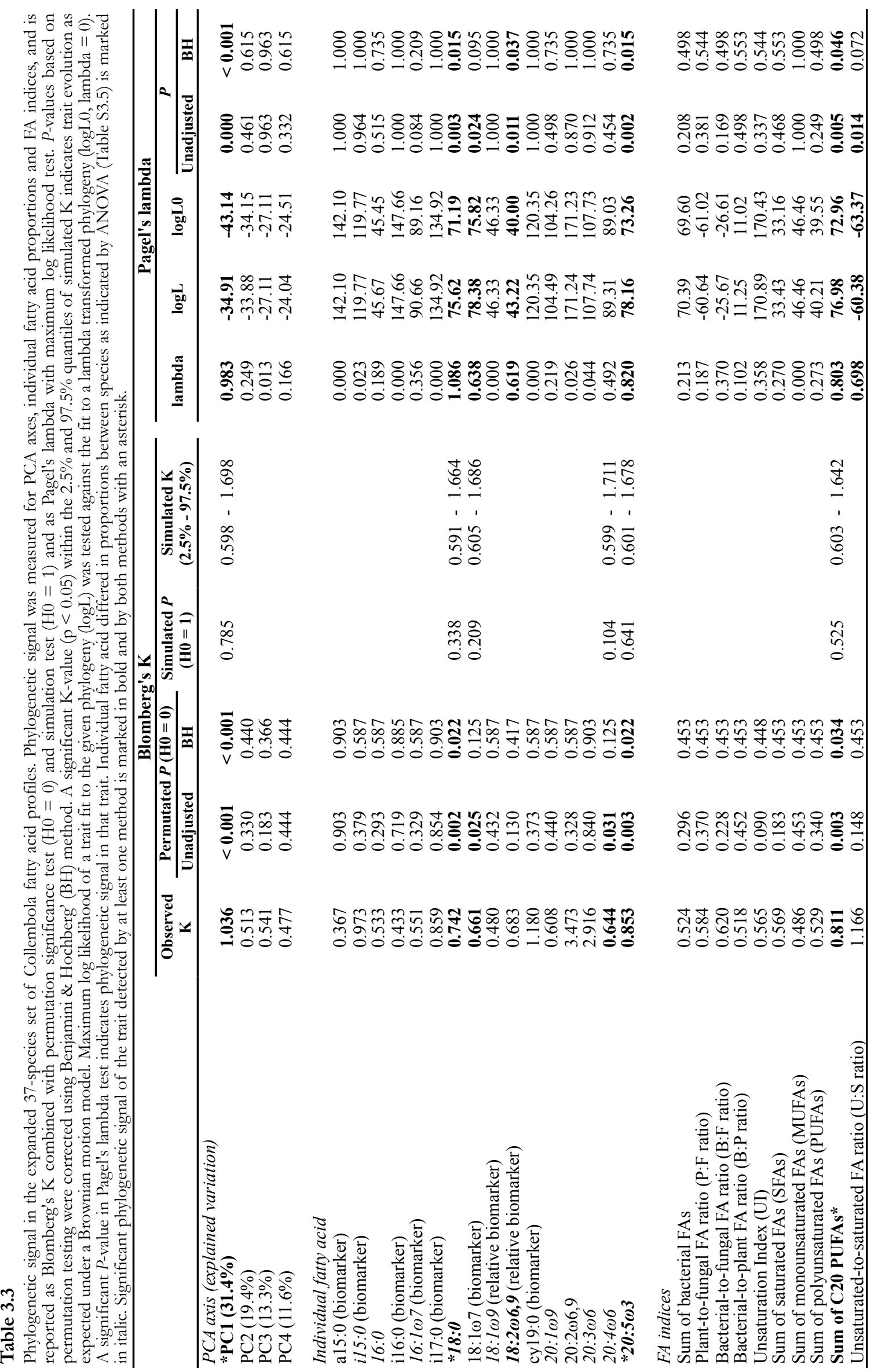




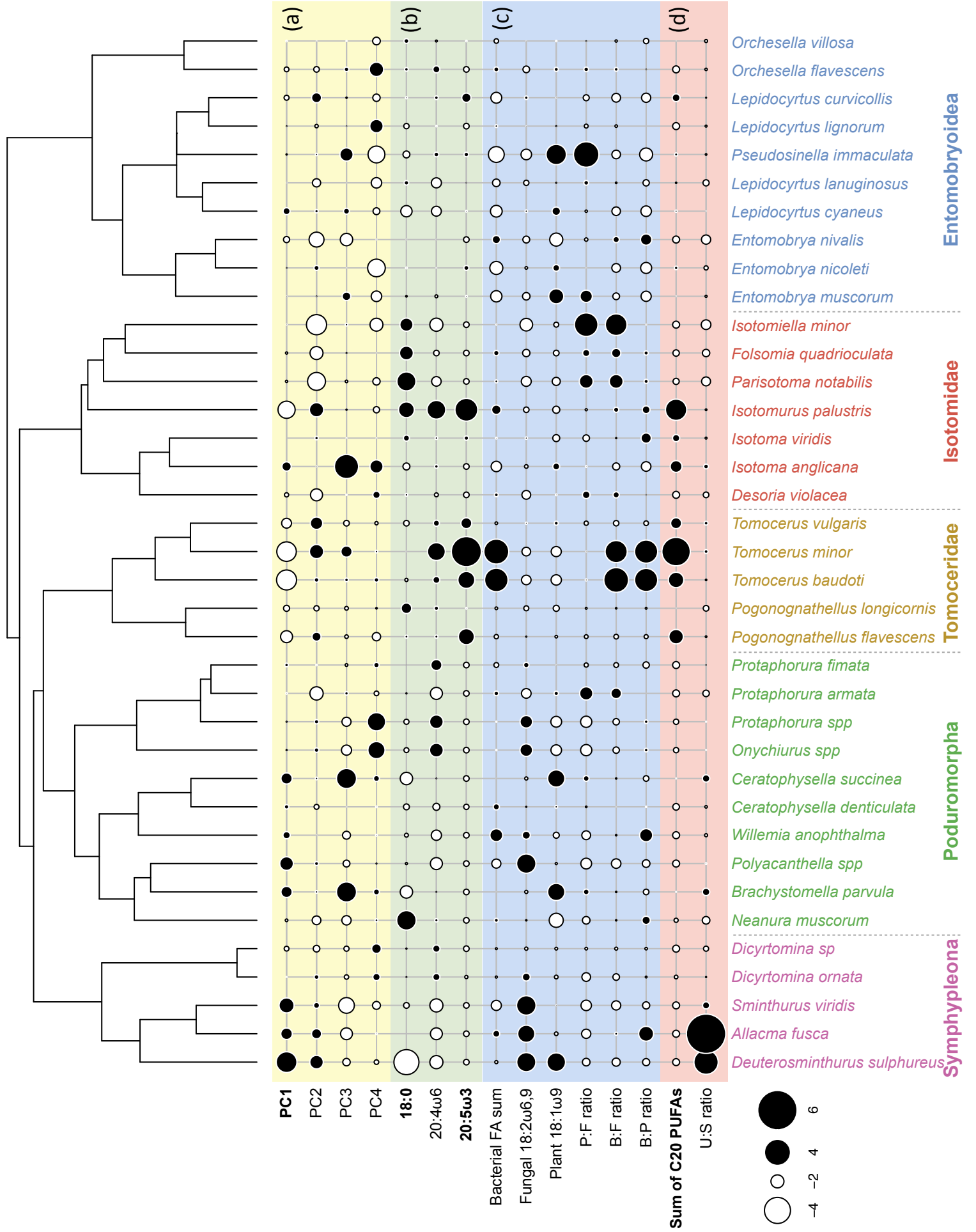

Figure 3.5

Relationship between phylogeny and selected trait values of Collembola using the combined dataset. Trait values were scaled and centralized before plotting. The size of the white and black circles indicate more negative or positive values, respectively. (a) Mean values of the scores of fatty acid profiles on the first four axes in principle components analysis (PCA), (b) proportions of fatty acids, (c) proportions of fatty acids derived from bacteria, fungi or plants, as well as the ratios between these three, and (d) summed proportions of C20 polyunsaturated fatty acid and ratio of unsaturated to saturated FAs; see Table 3.3 for abbreviations. Traits exhibiting phylogenetic signal as indicated by both Blomberg's $\mathrm{K}$ and Pagel's lambda are marked in bold. 


\section{Discussion}

Studies of FAs in Collembola usually have used one or two species in laboratory cultures under different conditions (Chamberlain et al. 2005, Haubert et al. 2008, van Dooremalen and Ellers 2010) or analyzed FAs of field-sampled species but with limited numbers of species sampled from one habitat type, i.e., forest (Chamberlain and Black 2005, Ruess et al. 2007, Ferlian et al. 2015) or arable fields (Haubert et al. 2009, Ngosong et al. 2009, Sechi et al. 2014). This study is the first to measure phylogenetic signal in FA compositions of field-sampled Collembola from different habitats using a phylogenetic comparative method. Our results suggest that although habitat effects on FA profiles were minor, FA compositions differed significantly between species and generally displayed phylogenetic signal, as indicated by the first PC axis for both field-sampled and combined datasets.

\section{Fatty acids, animal physiology and phylogenetics ( $\beta$ niche traits)}

Phylogenetic signal was detected in C20 PUFAs and proportions of 20:503 in both our field-sampled and combined datasets, supporting the first hypothesis that closely related Collembola species have similar proportions of C20 PUFAs. In field-sampled Collembola, Symphypleona contained lower proportions of C20 PUFAs than Entomobryomorpha, consistent with previous findings (Chamberlain and Black 2005). Collembola may have the ability to synthesize C20 PUFAs from precursors, as indicated by laboratory experiments in which a high proportion of C20 PUFAs was found in Isotomidae and Onychiuridae fed with food containing no PUFAs (Chamberlain and Black 2005). In insects, C20 PUFAs are essential for biosynthesis of prostaglandins and eicosanoids, which are important for reproduction and immune response, and related to temperature and humidity of the habitat (Stanley-Samuelson et al. 1992, Stanley-Samuelson 1994). Accordingly, the phylogenetic signal of C20 PUFA in different Collembola lineages presumably reflects an evolutionary constraint of physiological functions related to these FAs. Symphypleona predominantly live at the soil surface where humidity fluctuates with some dry periods, while the other taxa, such as Isotomidae, Tomoceridae and Poduromorpha, predominantly dwell in soil where humidity is high and relatively stable. Physiological constraints on the proportions of C20 PUFAs within phylogenetic lineages likely reflect the different soil horizons the species live in. However, the linkage between C20 PUFAs and the adaptation of species to different soil layers requires further examination of the functions of C20 PUFAs in Collembola.

\section{Fatty acids, food resources and phylogenetics ( $\alpha$ niche traits)}

Among biomarker FAs, only three markers $(18: 1 \omega 7,18: 2 \omega 6,9$ and 20:1 $1 \omega 9)$ exhibited phylogenetic signal in the field-sampled dataset, while the combined dataset showed phylogenetic signal in PC1 that

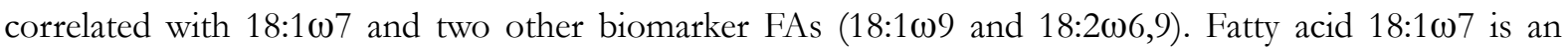
absolute bacterial biomarker synthesized exclusively by bacteria (Ruess and Chamberlain 2010, Ferlian et 
al. 2015). High proportions of 18:1 $1 \omega 7$ in Tomoceridae of our field-sampled dataset indicate that they fed heavily on bacteria at the study sites, whereas Entomobryoidea and Symphypleona consumed less of food resources containing this FA. Presumably, feeding on bacteria has been restricted to certain Collembola phylogenetic groups during evolutionary history, but this hypothesis needs further testing.

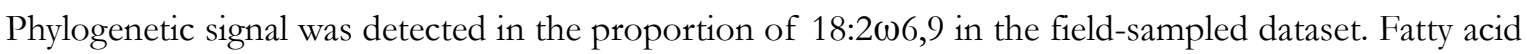

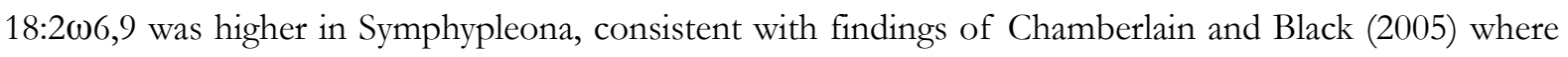
two Symphypleona species also had higher proportions of it than the other species sampled from a

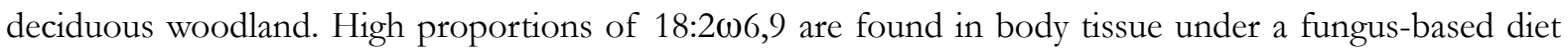
and thus have been used as indicator of fungal food resources (Ruess and Chamberlain 2010, Ferlian et al.

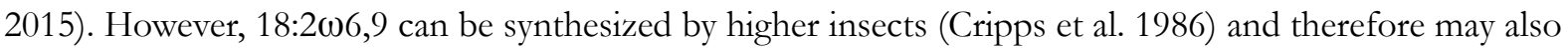

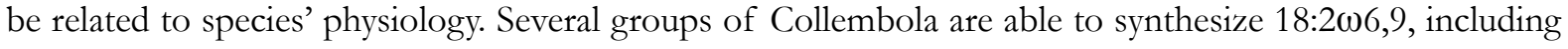
Isotomidae, Poduromorpha and Entomobryoidea (Chamberlain et al. 2004, Chamberlain and Black 2005, Haubert et al. 2006), but this has not been tested for Symphypleona. Nevertheless, high proportions of 18:2w6,9 still may reflect a fungal based diet in Symphypleona (Ruess et al. 2005, Ruess and Chamberlain 2010), but biosynthesis must be excluded by laboratory experiments before concluding that there is an evolutionary constraint in fungal feeding among different Collembola phylogenetic groups.

Strong phylogenetic signal was detected in the proportion of 20:109 of the field-sampled dataset. Collembola unlikely are able to biosynthesize 20:1 109 de novo but rather incorporate it from food, presumably from nematodes (Ruess et al. 2004, Ruess et al. 2005). The lack of 20:169 in the clade of Lepidocyrtidae and Entomobryidae and the clade of Sminthuridae indicates that at our study sites these Collembola did not feed on nematodes, while the remaining species, especially $C$. denticulate, may have consumed nematodes. However, when more species and measurements were included from other studies (the combined dataset), no phylogenetic signal was found in proportion of 20:1 109 , nor in site scores on PC3 and PC4 which were correlated with 20:1 $\omega 9$. Phylogenetic signal found in our field-sampled dataset may therefore be an exception. Indeed, Collembola from different forest sites have been shown with different proportions of 20:1 $1 \omega 9$, presumably related to the amounts of resources in the environment (Ruess et al. 2005).

The ratios of bacterial, fungal and plant FAs, which have been used to assign species to feeding guilds, did not show phylogenetic signal. These results partially support our second hypothesis that food resource FAs are a phylogenetically independent trait, implying niche partitioning in food resources among closely related species, thereby favoring species coexistence. Phylogenetic signal may be reduced due to a mixture of convergent evolution and conservatism in traits, or a developed trait irrespective of species' evolution (i.e., a phylogenetically random trait). Our analyses used ratio as a continuous variable and the ability to detect phylogenetic signal may be reduced due to large intraspecific variation or measurement errors (Ives et al. 2007). Indeed, Collembola are described as generalists able to consume a broad spectrum of food resources, exhibiting a considerable intraspecific variation in biomarker FA 
proportions from laboratory experiments (Chamberlain et al. 2005, Ruess et al. 2005, Haubert et al. 2011). In field samples, the variation is expected to be even larger, and it is possible that consumption of food resources is influenced by other co-occurring species.

Fatty acid composition complements stable isotopes in analyzing the trophic niche of soil biota (Ferlian et al. 2015). Using taxonomy as a surrogate of phylogenetic relationships with stable isotope data suggests conservatism in Collembola trophic niches (Potapov et al. 2016), in contrast to the findings of the current study. Thus, Collembola feeding traits are, on one hand, likely to have been constrained along species' evolutionary history; on the other hand, they may retain variability to reduce competition. More data on trophic niches and food resources of Collembola species from different phylogenetic groups are necessary to test this hypothesis.

\section{Traits and species coexistence in soil}

Species can coexist when they have similar $\beta$ niche traits and different $\alpha$ niche traits (Silvertown et al. 2006). Phylogenetic signal detected in C20 PUFAs ( $\beta$ niche) but general lability in biomarker FAs and bacterial, fungal and plant FA ratios ( $\alpha$ niche) may explain how different Collembola species coexist. Moreover, explicitly testing phylogenetic conservatism in functional traits is crucial for community phylogenetic and trait-based approaches, because the traits are mechanistic links by which phylogenetic history can influence contemporary ecological processes in communities (Cavender-Bares et al. 2009). Phylogenetic signal measurement in this study, therefore, represents a starting point to further investigate evolutionary hypotheses on the adaptation of soil animals to environmental conditions (Revell et al. 2008, Cooper et al. 2010), thereby linking community phylogenetic and trait-based approaches with coexistence studies on soil biota.

\section{Conclusions}

Our results show that Collembola FA profiles generally exhibit phylogenetic signal. We found phylogenetic signal in C20 PUFA proportions of Collembola, while biomarker FAs differed among species but were generally labile. These patterns suggest that (1) physiological properties of species may be constrained during evolutionary history, resulting in phylogenetically related species having similar physiologically related FAs, and (2) Collembola food resources are phylogenetically labile, favoring species coexistence. Our study is the first to report phylogenetic signal in the fatty acid compositions of animals in the context of species coexistence. The results form a starting point to further investigate evolutionary hypotheses on the adaptation of soil animals to environmental conditions. Integrating phylogenetic comparative methods and community phylogenetic and trait-based approaches may help identify evolutionary and ecological forces driving and maintaining communities in soil. 


\section{Acknowledgements}

We thank Olga Ferlian, Dominique Haubert, Christopher Ngosong, Melanie M. Pollierer and Liliane Ruess for providing their data on Collembola fatty acids. We acknowledge the associate editor and three anonymous reviewers for their constructive comments. Special thanks to Guido Humpert and Jo-Fan Chao for support during lab work and Tamara Hartke for improving the English. The authors have no conflict of interest to declare.

\section{Author Contributions}

TWC and SS conceived and designed the study; TWC and PS performed the study; TWC, IS and SS wrote the manuscript. All authors revised and approved the manuscript.

\section{Data Accessibility}

Concatenated alignment and phylogenetic trees generated from this study were deposited in TreeBASE (http://purl.org/phylo/treebase/phylows/study/TB2:S20409).

\section{References}

Ackerly, D.D. \& Cornwell, W.K. (2007) A trait-based approach to community assembly: Partitioning of species trait values into within- and among-community components. Ecology Letters, 10, 135-145.

Ackerly, D.D., Schwilk, D.W. \& Webb, C.O. (2006) Niche evolution and adaptive radiation: testing the order of trait divergence. Ecology, 87, S50-61.

Adler, P.B., Fajardo, A., Kleinhesselink, A.R. \& Kraft, N.J.B. (2013) Trait-based tests of coexistence mechanisms. Ecology Letters, 16, 1294-1306.

Benjamini, Y. \& Hochberg, Y. (1995) Controlling the false discovery rate: a practical and powerful approach to multiple testing. Journal of the Royal Statistical Society Series B, 57, 289-300.

Best, R.J. \& Stachowicz, J.J. (2013) Phylogeny as a proxy for ecology in seagrass amphipods: which traits are most conserved? PLOS ONE, 8, e57550.

Blomberg, S.P., Garland Jr., T. \& Ives, A.R. (2003) Testing for phylogenetic signal in comparative data: Behavioral traits are more labile. Evolution, 57, 717-745.

Bodenhofer, U., Bonatesta, E., Horejs-Kainrath, C. \& Hochreiter, S. (2015) msa: An R package for multiple sequence alignment. Bioinformatics, 31, 3997-3999.

Buse, T., Ruess, L. \& Filser, J. (2013) New trophic biomarkers for Collembola reared on algal diets. Pedobiologia, 56, 153-159.

Cavender-Bares, J., Ackerly, D.D., Baum, D.A. \& Bazzaz, F.A. (2004) Phylogenetic overdispersion in Floridian oak communities. The American Naturalist, 163, 823-843.

Cavender-Bares, J., Kozak, K.H., Fine, P. V \& Kembel, S.W. (2009) The merging of community ecology and phylogenetic biology. Ecology Letters, 12, 693-715. 
Chamberlain, P.M. \& Black, H.I.J. (2005) Fatty acid compositions of Collembola: unusually high proportions of C20 polyunsaturated fatty acids in a terrestrial invertebrate. Comparative Biochemistry and Physiology Part B: Biochemistry and Molecular Biology, 140, 299-307.

Chamberlain, P.M., Bull, I.D., Black, H.I., Ineson, P. \& Evershed, R.P. (2004) Lipid content and carbon assimilation in Collembola: implications for the use of compound-specific carbon isotope analysis in animal dietary studies. Oecologia, 139, 325-335.

Chamberlain, P.M., Bull, I.D., Black, H.I.J., Ineson, P. \& Evershed, R.P. (2005) Fatty acid composition and change in Collembola fed differing diets: identification of trophic biomarkers. Soil Biology and Biochemistry, 37, 1608-1624.

Cooper, N., Jetz, W. \& Freckleton, R.P. (2010) Phylogenetic comparative approaches for studying niche conservatism. Journal of Evolutionary Biology, 23, 2529-2539.

Cripps, C., Blomquist, G.J. \& de Renobales, M. (1986) De novo biosynthesis of linoleic acid in insects. Biochimica et Biophysica Acta (BBA) - Lipids and Lipid Metabolism, 876, 572-580.

Darriba, D., Taboada, G.L., Doallo, R. \& Posada, D. (2012) jModelTest 2: more models, new heuristics and parallel computing. Nature Methods, 9, 772.

van Dooremalen, C. \& Ellers, J. (2010) A moderate change in temperature induces changes in fatty acid composition of storage and membrane lipids in a soil arthropod. Journal of Insect Physiology, 56, 178-184.

Ferlian, O., Klarner, B., Langeneckert, A.E. \& Scheu, S. (2015) Trophic niche differentiation and utilisation of food resources in collembolans based on complementary analyses of fatty acids and stable isotopes. Soil Biology and Biochemistry, 82, 28-35.

Ferlian, O. \& Scheu, S. (2014) Shifts in trophic interactions with forest type in soil generalist predators as indicated by complementary analyses of fatty acids and stable isotopes. Oikos, 123, 1182-1191.

Fox, J. \& Weisberg, S. (2011) An R Companion to Applied Regression, 2nd ed. Sage, Thousand Oaks, CA.

Freckleton, R.P., Harvey, P.H. \& Pagel, M. (2002) Phylogenetic analysis and comparative data. The American Naturalist, 160, 712-726.

Hall, T.A. (1999) BioEdit: a user-friendly biological sequence alignment editor and analysis program for Windows 95/98/NT. Nucleic Acids Symposium Series, 41, 95-98.

Harvey, P.H. \& Pagel, M.D. (1991) The Comparative Method in Evolutionary Biology. Oxford University Press, Oxford.

Haubert, D., Birkhofer, K., Fließbach, A., Gehre, M., Scheu, S. \& Ruess, L. (2009) Trophic structure and major trophic links in conventional versus organic farming systems as indicated by carbon stable isotope ratios of fatty acids. Oikos, 118, 1579-1589.

Haubert, D., Häggblom, M.M., Langel, R., Scheu, S. \& Ruess, L. (2006) Trophic shift of stable isotopes and fatty acids in Collembola on bacterial diets. Soil Biology and Biochemistry, 38, 2004-2007.

Haubert, D., Häggblom, M.M., Scheu, S. \& Ruess, L. (2004) Effects of fungal food quality and starvation on the fatty acid composition of Protaphorura fimata (Collembola). Comparative Biochemistry and Physiology Part B: Biochemistry and Molecular Biology, 138, 41-52.

Haubert, D., Häggblom, M.M., Scheu, S. \& Ruess, L. (2008) Effects of temperature and life stage on the fatty acid composition of Collembola. European Journal of Soil Biology, 44, 213-219.

Haubert, D., Pollierer, M.M. \& Scheu, S. (2011) Fatty acid patterns as biomarker for trophic interactions: Changes after dietary switch and starvation. Soil Biology and Biochemistry, 43, 490-494.

Holm, S. (1979) A simple sequentially rejective multiple test procedure. Scandinavian Journal of Statistics, 6, 65-70.

Hopkin, S.P. (1997) Biology of the Springtails (Insecta: Collembola). Oxford University Press, Oxford. 
Hopkin, S.P. (2007) A Key to the Collembola (Springtails) of Britain and Ireland (ed Field Studies Council (FSC)). Shrewsbury.

Ives, A.R., Midford, P.E. \& Garland, T. (2007) Within-species variation and measurement error in phylogenetic comparative methods. Systematic Biology, 56, 252-270.

Kempson, D., Lloyd, M. \& Ghelardi, R. (1963) A new extractor for woodland litter. Pedobiologia, 3, 1-21.

McGill, B.J., Enquist, B.J., Weiher, E. \& Westoby, M. (2006) Rebuilding community ecology from functional traits. Trends in Ecology \& Evolution, 21, 178-185.

Münkemüller, T., Lavergne, S., Bzeznik, B., Dray, S., Jombart, T., Schiffers, K. \& Thuiller, W. (2012) How to measure and test phylogenetic signal. Methods in Ecology and Evolution, 3, 743-756.

Ngosong, C., Raupp, J., Scheu, S. \& Ruess, L. (2009) Low importance for a fungal based food web in arable soils under mineral and organic fertilization indicated by Collembola grazers. Soil Biology and Biochemistry, 41, 23082317.

Ngosong, C., Raupp, J., Richnow, H., \& Ruess, L. (2011). Tracking Collembola feeding strategies by the natural ${ }^{13} \mathrm{C}$ signal of fatty acids in an arable soil with different fertilizer regimes. Pedobiologia, 54, 225-233.

Pagel, M. (1999) Inferring the historical patterns of biological evolution. Nature, 401, 877-884.

Paradis, E., Claude, J. \& Strimmer, K. (2004) APE: Analyses of phylogenetics and evolution in R language. Bioinformatics, 20, 289-290.

Paradis, E. (2013) Molecular dating of phylogenies by likelihood methods: A comparison of models and a new information criterion. Molecular Phylogenetics and Evolution, 67, 436-444.

Pey, B., Nahmani, J., Auclerc, A., Capowiez, Y., Cluzeau, D., Cortet, J., Decaëns, T., Deharveng, L., Dubs, F., Joimel, S., Briard, C., Grumiaux, F., Laporte, M.-A.A., Pasquet, A., Pelosi, C., Pernin, C., Ponge, J.-F., Salmon, S., Santorufo, L. \& Hedde, M. (2014) Current use of and future needs for soil invertebrate functional traits in community ecology. Basic and Applied Ecology, 15, 194-206.

Pollierer, M.M., Dyckmans, J., Scheu, S. \& Haubert, D. (2012) Carbon flux through fungi and bacteria into the forest soil animal food web as indicated by compound-specific ${ }^{13} \mathrm{C}$ fatty acid analysis. Functional Ecology, 26, 978-990.

Potapov, A.A., Semenina, E.E., Korotkevich, A.Y., Kuznetsova, N.A. \& Tiunov, A. V. (2016) Connecting taxonomy and ecology: Trophic niches of collembolans as related to taxonomic identity and life forms. Soil Biology and Biochemistry, 101, 20-31.

R Core Team. (2015) R: A language and environment for statistical computing.

Revell, L.J. (2012) phytools: An R package for phylogenetic comparative biology (and other things). Methods in Ecology and Evolution, 3, 217-223.

Revell, L., Harmon, L. \& Collar, D. (2008) Phylogenetic signal, evolutionary process, and rate. Systematic Biology, 57, 591-601.

Revell, L.J., Johnson, M.A., Schulte, J.A., Kolbe, J.J. \& Losos, J.B. (2007) A phylogenetic test for adaptive convergence in rock-dwelling lizards. Evolution, 61, 2898-2912.

Ronquist, F., Teslenko, M., van Der Mark, P., Ayres, D.L., Darling, A., Höhna, S., Larget, B., Liu, L., Suchard, M.A. \& Huelsenbeck, J.P. (2012) Mrbayes 3.2: Efficient bayesian phylogenetic inference and model choice across a large model space. Systematic Biology, 61, 539-542.

Ruess, L. \& Chamberlain, P.M. (2010) The fat that matters: Soil food web analysis using fatty acids and their carbon stable isotope signature. Soil Biology and Biochemistry, 42, 1898-1910.

Ruess, L., Häggblom, M.M., Langel, R. \& Scheu, S. (2004) Nitrogen isotope ratios and fatty acid composition as indicators of animal diets in belowground systems. Oecologia, 139, 336-346. 
Ruess, L., Schütz, K., Haubert, D., Häggblom, M.M., Kandeler, E. \& Scheu, S. (2005) Application of lipid analysis to understand trophic interactions in soil. Ecology, 86, 2075-2082.

Ruess, L., Schütz, K., Migge-Kleian, S., Häggblom, M.M., Kandeler, E. \& Scheu, S. (2007) Lipid composition of Collembola and their food resources in deciduous forest stands-Implications for feeding strategies. Soil Biology and Biochemistry, 39, 1990-2000.

Rusek, J. (1998) Biodiversity of Collembola and their functional role in the ecosystem. Biodiversity and Conservation, 7 , 1207-1219.

Sechi, V., D’Annibale, A., Ambus, P., Sárossy, Z., Krogh, P.H., Eriksen, J. \& Holmstrup, M. (2014) Collembola feeding habits and niche specialization in agricultural grasslands of different composition. Soil Biology and Biochemistry, 74, 31-38.

Silvertown, J., McConway, K., Gowing, D., Dodd, M., Fay, M.F., Joseph, J.A. \& Dolphin, K. (2006) Absence of phylogenetic signal in the niche structure of meadow plant communities. Proceedings of the Royal Society B, 273, 3944.

Stamatakis, A. (2006) RAxML-VI-HPC: Maximum likelihood-based phylogenetic analyses with thousands of taxa and mixed models. Bioinformatics, 22, 2688-2690.Stanley-Samuelson, D.W. (1994) Assessing the significance of prostaglandins and other eicosanoids in insect physiology. Journal of Insect Physiology, 40, 3-11.

Stanley-Samuelson, D.W., Dell, T. \& Ogg, C.L. (1992) Polyunsaturated fatty acid metabolism inferred from fatty acid compositions of the diets and tissues of the gypsy moth Lymantria dispar. Comparative Biochemistry and Physiology, 102A, 173-178.

Vaidya, G., Lohman, D.J. \& Meier, R. (2011) SequenceMatrix: Concatenation software for the fast assembly of multi-gene datasets with character set and codon information. Cladistics, 27, 171-180.

Venables, W.N. \& Ripley, B.D. (2002) Modern Applied Statistics with S, 4th ed. Springer, New York.

Violle, C., Navas, M.L., Vile, D., Kazakou, E., Fortunel, C., Hummel, I. \& Garnier, E. (2007) Let the concept of trait be functional! Oikos, 116, 882-892.

Webb, C.O., Ackerly, D.D., McPeek, M.A. \& Donoghue, M.J. (2002) Phylogenies and community ecology. Annual Review of Ecology and Systematics, 33, 475-505.

Wright, E.S. (2015) DECIPHER: harnessing local sequence context to improve protein multiple sequence alignment. BMC Bioinformatics, 16, 322. 


\section{Supplementary Materials}

Table S3.1

NCBI accession numbers of sequences used to construct the molecular phylogeny of Collembola.

\begin{tabular}{|c|c|c|c|c|c|c|}
\hline \multirow{3}{*}{ Species } & \multicolumn{6}{|c|}{ Molecular markers } \\
\hline & \multirow{2}{*}{ 18S rRNA } & \multicolumn{3}{|c|}{ 28S rRNA } & \multirow{2}{*}{ Histone $\mathrm{H3}$} & \multirow{2}{*}{ COI } \\
\hline & & D1 & D2 & D3-D5 & & \\
\hline Zygentoma (outgroup) & AF370791 & AY859557 & AY859557 & AY859557 & AY749703 & KM535783 \\
\hline Callibaetis (outgroup) & AY521826 & AY521735 & AY521735 & AY521735 & AY521695 & JF735107 \\
\hline Machilis (outgroup) & AY210811 & AY210810 & AY210810 & AY210810 & AY338644 & JN970940 \\
\hline Allacma fusca & KY230702 & KY230832 & KY230832 & KY230935 & & KY231097 \\
\hline Brachystomella parvula & KY230724 & KY230822 & KY230822 & KY230925 & KY231066 & KY231088 \\
\hline Ceratophysella denticulata & KY230747 & KY230847 & KY230847 & KY230948 & KY231036 & KY231107 \\
\hline Ceratophysella succinea & & KY230885 & KY230885 & KY230990 & KY231065 & KY231136 \\
\hline Desoria violacea & KY230736 & KY230833 & KY230833 & KY230902 & KY231003 & KY231098 \\
\hline Deuterosminthurus sulphureus & KY230753 & KY230830 & KY230830 & KY230933 & KY231024 & KY231095 \\
\hline Dicyrtomina ornata & KY230768 & KY230840 & KY230840 & KY230968 & KY231046 & KY231125 \\
\hline Dicyrtomina saundersi & EU368611 & EF199974 & EF199974 & EF199974 & & \\
\hline Entomobrya muscorum & KY230710 & KY230806 & KY230806 & KY230909 & KY231010 & \\
\hline Entomobrya nicoleti & KY230740 & KY230838 & KY230838 & KY230941 & KY231030 & KY231119 \\
\hline Entomobrya nivalis & & LK024313 & LK024313 & & & HG422598 \\
\hline Folsomia quadrioculata & KY230755 & KY230853 & KY230853 & KY230955 & KF684772 & KF684607 \\
\hline Friesea claviseta & KY230727 & KY230826 & KY230826 & KY230929 & KY231020 & KY231092 \\
\hline Isotoma anglicana & KY230779 & KY230873 & KY230873 & KY230975 & KY231053 & KY231076 \\
\hline Isotoma viridis & KY230708 & KY230835 & KY230835 & KY230938 & KY231028 & KY231129 \\
\hline Isotomiella minor & KY230744 & KY230843 & KY230843 & KY230945 & KY231034 & KY231103 \\
\hline Isotomurus fucicolus & KY230704 & KY230834 & KY230834 & KY230937 & KY231057 & KY231099 \\
\hline Lepidocyrtus cyaneus & KY230751 & KY230851 & KY230851 & KY230952 & KY231040 & KY231111 \\
\hline Lepidocyrtus lanuginosus & KY230748 & KY230848 & KY230848 & KY230949 & KY231037 & KY231108 \\
\hline Lepidocyrtus lignorum & KY230749 & KY230849 & KY230849 & KY230950 & KY231038 & KY231109 \\
\hline Lepidocyrtus sp & KY230750 & KY230850 & KY230850 & KY230951 & KY231039 & KY231110 \\
\hline Neanura muscorum & AY555520 & AJ251733 & AJ 251733 & AJ 251733 & & AY555544 \\
\hline Onychiurus ambulans & AY555518 & AF483384 & AF483442 & HQ731961 & AY555564 & HQ732075 \\
\hline Orchesella flavescens & KY230714 & KY230811 & KY230811 & KY230997 & KY231015 & KY231082 \\
\hline Orchesella villosa & KY230715 & KY230812 & KY230812 & KY230904 & KY231005 & KY231091 \\
\hline Parisotoma notabilis & KY230772 & KY230872 & KY230872 & KY230974 & KY231052 & KY231128 \\
\hline Pogonognathellus flavescens & KY230717 & KY230814 & KY230814 & KY230917 & KY231016 & KY231083 \\
\hline Pogonognathellus longicornis & & KY230845 & KY230845 & KY230946 & KY231035 & KY231105 \\
\hline Protaphorura armata & & AF483391 & AF483449 & HQ731965 & & HQ732078 \\
\hline Protaphorura spl & KY230789 & KY230884 & KY230884 & KY230989 & KY231063 & HG422585 \\
\hline Protaphorura sp 2 & KY230719 & KY230856 & KY230856 & KY230959 & KY231064 & KY231116 \\
\hline Pseudosinella immaculata & KY230712 & & & KY230911 & KY231012 & KY231078 \\
\hline Sminthurus viridis & KY230701 & KY230880 & KY230880 & KY230983 & KY231001 & JN970939 \\
\hline Tomocerus baudoti & & & JX261697 & & & JX261845 \\
\hline Tomocerus minor & AY555516 & AF483406 & JX261700 & HQ731971 & AY555562 & HM398041 \\
\hline Tomocerus vulgaris & KY230777 & KY230815 & KY230815 & KY230918 & KY231058 & KY231131 \\
\hline Willemia anophthalma & KY230726 & KY230869 & KY230869 & KY230927 & & KY231090 \\
\hline
\end{tabular}




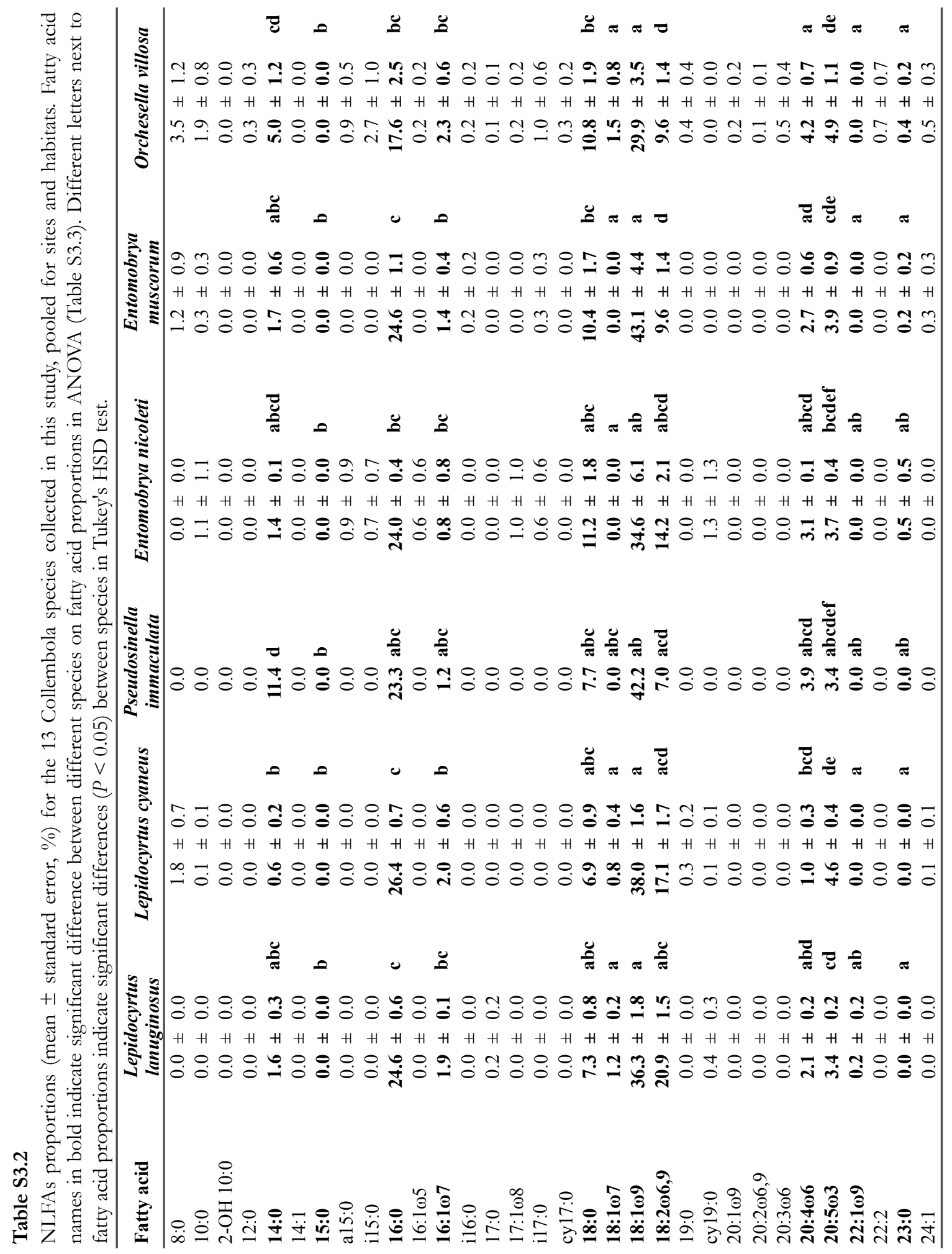




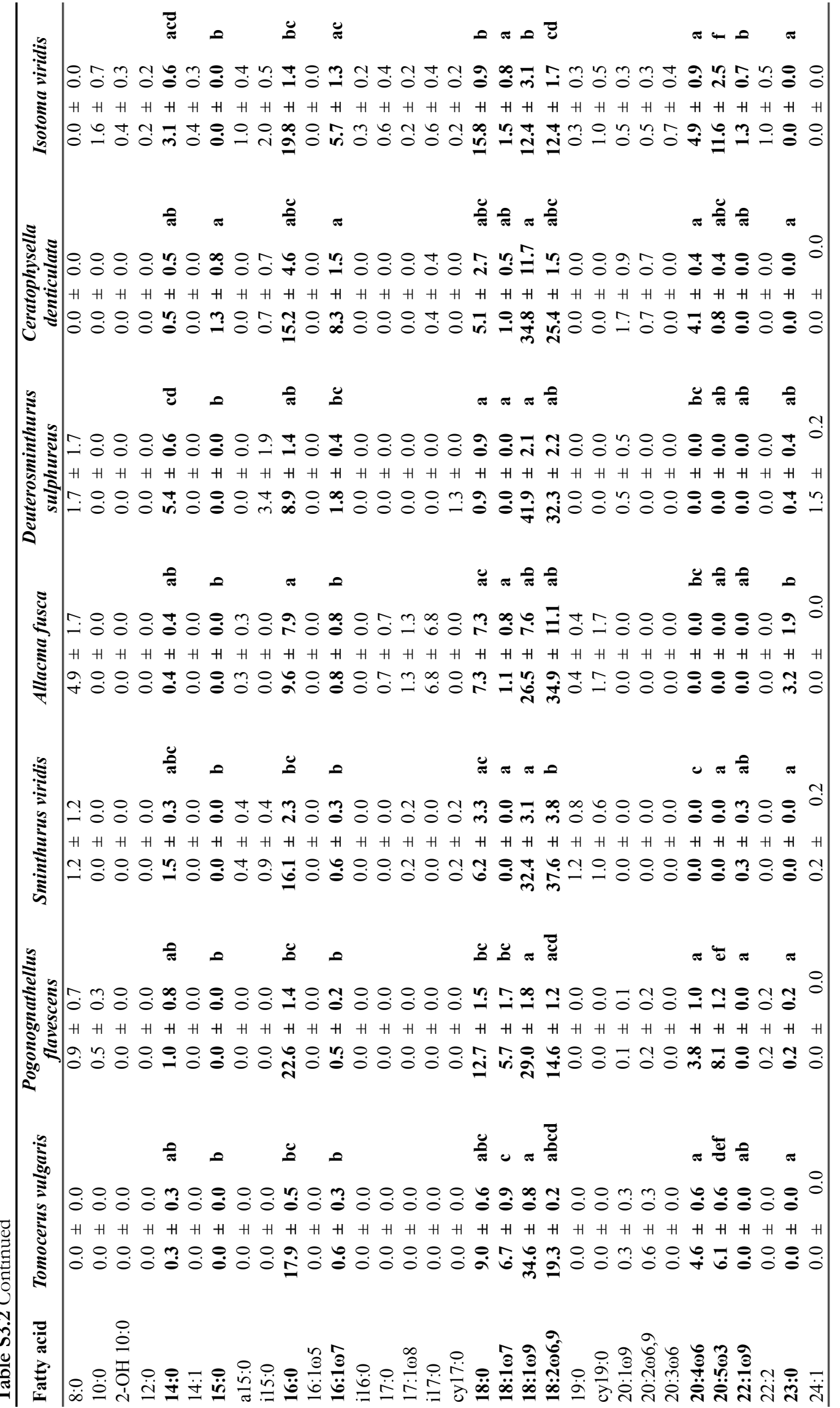




\section{Table S3.3}

ANOVA table of logit-transformed proportions for each fatty acid from 13 field-collected Collembola species. Species and habitats are used as explanatory factors and sites and habitats as error terms in the model. $P$-values were obtained after Holm's correction. Fatty acids significantly different between species are marked in bold.

\begin{tabular}{|c|c|c|c|c|c|c|}
\hline \multirow{2}{*}{ Fatty acid } & \multicolumn{3}{|c|}{ Species } & \multicolumn{3}{|c|}{ Habitat:Species } \\
\hline & df & $\mathbf{F}$ & Adjusted $P$ & df & $\mathbf{F}$ & Adjusted $P$ \\
\hline 8:0 & 12 & 2.111 & 0.539 & 8 & 1.077 & 0.975 \\
\hline 10:0 & 12 & 1.622 & 1.000 & 8 & 1.083 & 0.975 \\
\hline 2-OH 10:0 & 12 & 2.050 & 0.539 & 8 & 2.288 & 0.211 \\
\hline $12: 0$ & 12 & 0.182 & 1.000 & 8 & 0.235 & 1.000 \\
\hline 14:0 & 12 & 5.324 & 0.001 & 8 & 2.063 & 0.277 \\
\hline $14: 1$ & 12 & 2.258 & 0.423 & 8 & 2.520 & 0.193 \\
\hline $15: 0$ & 12 & 4.630 & 0.002 & 8 & 0.000 & 1.000 \\
\hline a15:0 & 12 & 1.364 & 1.000 & 8 & 1.895 & 0.340 \\
\hline i15:0 & 12 & 2.894 & 0.100 & 8 & 1.331 & 0.738 \\
\hline 16:0 & 12 & 4.759 & 0.002 & 8 & 0.214 & 1.000 \\
\hline $16: 1 \omega 5$ & 12 & 1.336 & 1.000 & 8 & 0.091 & 1.000 \\
\hline 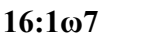 & 12 & 6.120 & $<0.001$ & 8 & 1.470 & 0.626 \\
\hline i16:0 & 12 & 0.558 & 1.000 & 8 & 0.629 & 1.000 \\
\hline 17:0 & 12 & 0.954 & 1.000 & 8 & 0.934 & 1.000 \\
\hline $17: 1 \omega 8$ & 12 & 1.135 & 1.000 & 8 & 0.623 & 1.000 \\
\hline i17:0 & 12 & 0.894 & 1.000 & 8 & 1.003 & 1.000 \\
\hline cy17:0 & 12 & 2.150 & 0.521 & 8 & 1.660 & 0.482 \\
\hline 18:0 & 12 & 3.657 & 0.018 & 8 & 0.901 & 1.000 \\
\hline 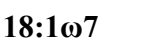 & 12 & 4.975 & 0.001 & 8 & 0.855 & 1.000 \\
\hline 18:109 & 12 & 4.761 & 0.002 & 8 & 0.420 & 1.000 \\
\hline $18: 2 \omega 6,9$ & 12 & 6.162 & $<0.001$ & 8 & 0.705 & 1.000 \\
\hline $19: 0$ & 12 & 2.571 & 0.214 & 8 & 2.514 & 0.193 \\
\hline cy19:0 & 12 & 2.508 & 0.237 & 8 & 2.272 & 0.211 \\
\hline 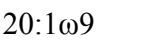 & 12 & 2.062 & 0.539 & 8 & 0.278 & 1.000 \\
\hline $20: 2 \omega 6,9$ & 12 & 0.962 & 1.000 & 8 & 0.087 & 1.000 \\
\hline $20: 3 \omega 6$ & 12 & 0.734 & 1.000 & 8 & 0.272 & 1.000 \\
\hline $20: 4 \omega 6$ & 12 & 8.411 & $<0.001$ & 8 & 0.354 & 1.000 \\
\hline $20: 5 \omega 3$ & 12 & 16.549 & $<0.001$ & 8 & 0.354 & 1.000 \\
\hline 22:109 & 12 & 3.519 & 0.023 & 8 & 2.538 & 0.193 \\
\hline $22: 2$ & 12 & 0.688 & 1.000 & 8 & 0.043 & 1.000 \\
\hline 23:0 & 12 & 3.663 & 0.018 & 8 & 2.642 & 0.193 \\
\hline $24: 1$ & 12 & 2.109 & 0.539 & 8 & 0.493 & 1.000 \\
\hline
\end{tabular}




\section{Table S3.4}

Correlations of different fatty acids with the first four axes (PCs) from principle component analyses. $P$-values were adjusted using Holm's method. Fatty acids correlated with the first PC are marked in bold.

\begin{tabular}{|c|c|c|c|c|c|c|c|c|}
\hline \multirow{2}{*}{ Fatty acid } & \multicolumn{2}{|c|}{ PC1 } & \multicolumn{2}{|c|}{ PC2 } & \multicolumn{2}{|c|}{ PC3 } & \multicolumn{2}{|c|}{ PC4 } \\
\hline & *cor & $\boldsymbol{P}$ & cor & $P$ & cor & $\boldsymbol{P}$ & cor & $\boldsymbol{P}$ \\
\hline \multicolumn{9}{|c|}{ Field-derived species } \\
\hline $8: 0$ & 0.483 & 1.000 & 0.121 & 1.000 & -0.225 & 1.000 & 0.649 & 0.476 \\
\hline $10: 0$ & -0.566 & 1.000 & 0.036 & 1.000 & -0.575 & 0.832 & 0.248 & 1.000 \\
\hline 2-OH 10:0 & -0.404 & 1.000 & 0.224 & 1.000 & -0.697 & 0.220 & -0.121 & 1.000 \\
\hline $12: 0$ & -0.402 & 1.000 & 0.013 & 1.000 & -0.744 & 0.107 & 0.141 & 1.000 \\
\hline 14:0 & -0.117 & 1.000 & -0.850 & 0.008 & -0.361 & 1.000 & 0.074 & 1.000 \\
\hline $14: 1$ & -0.404 & 1.000 & 0.224 & 1.000 & -0.697 & 0.220 & -0.121 & 1.000 \\
\hline $15: 0$ & 0.161 & 1.000 & 0.151 & 1.000 & -0.125 & 1.000 & -0.770 & 0.065 \\
\hline a15:0 & -0.259 & 1.000 & 0.103 & 1.000 & -0.656 & 0.360 & 0.277 & 1.000 \\
\hline i15:0 & 0.175 & 1.000 & -0.218 & 1.000 & -0.704 & 0.203 & -0.130 & 1.000 \\
\hline 16:0 & -0.778 & 0.048 & -0.235 & 1.000 & 0.422 & 1.000 & -0.178 & 1.000 \\
\hline $16: 1 \omega 5$ & -0.185 & 1.000 & -0.103 & 1.000 & -0.013 & 1.000 & 0.202 & 1.000 \\
\hline $16: 1 \omega 7$ & -0.095 & 1.000 & 0.033 & 1.000 & -0.547 & 1.000 & -0.751 & 0.092 \\
\hline i16:0 & -0.495 & 1.000 & -0.000 & 1.000 & -0.627 & 0.478 & 0.077 & 1.000 \\
\hline 17:0 & 0.073 & 1.000 & 0.413 & 1.000 & -0.669 & 0.311 & 0.352 & 1.000 \\
\hline $17: 1 \omega 8$ & 0.237 & 1.000 & 0.238 & 1.000 & -0.263 & 1.000 & 0.538 & 1.000 \\
\hline i17:0 & 0.298 & 1.000 & 0.339 & 1.000 & -0.486 & 1.000 & 0.518 & 1.000 \\
\hline cy17:0 & 0.484 & 1.000 & -0.354 & 1.000 & -0.321 & 1.000 & -0.073 & 1.000 \\
\hline 18:0 & -0.953 & $<0.001$ & 0.158 & 1.000 & -0.022 & 1.000 & 0.135 & 1.000 \\
\hline $18: 1 \omega 7$ & -0.357 & 1.000 & 0.674 & 0.358 & 0.252 & 1.000 & -0.003 & 1.000 \\
\hline $18: 1 \omega 9$ & 0.235 & 1.000 & -0.573 & 1.000 & 0.731 & 0.130 & -0.081 & 1.000 \\
\hline $18: 2 \omega 6,9$ & 0.849 & 0.007 & 0.372 & 1.000 & 0.087 & 1.000 & -0.231 & 1.000 \\
\hline $19: 0$ & 0.382 & 1.000 & 0.178 & 1.000 & -0.182 & 1.000 & 0.225 & 1.000 \\
\hline cy19:0 & 0.208 & 1.000 & 0.314 & 1.000 & -0.294 & 1.000 & 0.339 & 1.000 \\
\hline $20: 1 \omega 9$ & 0.122 & 1.000 & 0.195 & 1.000 & -0.331 & 1.000 & -0.805 & 0.029 \\
\hline $20: 2 \omega 6,9$ & -0.294 & 1.000 & 0.516 & 1.000 & -0.243 & 1.000 & -0.584 & 0.973 \\
\hline $20: 3 \omega 6$ & -0.452 & 1.000 & 0.104 & 1.000 & -0.814 & 0.022 & 0.047 & 1.000 \\
\hline $20: 4 \omega 6$ & -0.854 & 0.006 & 0.037 & 1.000 & -0.115 & 1.000 & -0.276 & 1.000 \\
\hline $20: 5 \omega 3$ & -0.948 & $<0.001$ & 0.181 & 1.000 & -0.019 & 1.000 & 0.069 & 1.000 \\
\hline $22: 1 \omega 9$ & -0.320 & 1.000 & 0.227 & 1.000 & -0.631 & 0.478 & -0.154 & 1.000 \\
\hline $22: 2$ & -0.508 & 1.000 & 0.189 & 1.000 & -0.774 & 0.059 & 0.042 & 1.000 \\
\hline $23: 0$ & 0.515 & 1.000 & 0.259 & 1.000 & -0.237 & 1.000 & 0.627 & 0.613 \\
\hline $24: 1$ & 0.493 & 1.000 & -0.429 & 1.000 & -0.171 & 1.000 & -0.029 & 1.000 \\
\hline \multicolumn{9}{|c|}{ Literature 37 species } \\
\hline a15:0 & -0.066 & 1.000 & -0.618 & 0.001 & -0.355 & 0.336 & -0.027 & 1.000 \\
\hline i15:0 & 0.148 & 1.000 & -0.240 & 1.000 & -0.300 & 0.569 & -0.246 & 0.878 \\
\hline 16:0 & -0.256 & 1.000 & -0.870 & $<0.001$ & -0.040 & 1.000 & -0.181 & 1.000 \\
\hline i16:0 & -0.215 & 1.000 & -0.461 & 0.045 & -0.219 & 1.000 & 0.290 & 0.820 \\
\hline $16: 1 \omega 7$ & 0.207 & 1.000 & 0.029 & 1.000 & 0.531 & 0.010 & 0.351 & 0.400 \\
\hline i17:0 & 0.150 & 1.000 & 0.028 & 1.000 & -0.214 & 1.000 & -0.189 & 1.000 \\
\hline 18:0 & -0.643 & $<0.001$ & -0.481 & 0.031 & -0.046 & 1.000 & -0.112 & 1.000 \\
\hline 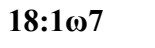 & -0.825 & $<0.001$ & -0.063 & 1.000 & -0.252 & 0.932 & 0.394 & 0.224 \\
\hline 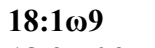 & 0.477 & 0.031 & 0.183 & 1.000 & 0.586 & 0.002 & -0.283 & 0.820 \\
\hline $18: 2 \omega 6,9$ & 0.542 & 0.006 & 0.572 & 0.003 & -0.513 & 0.014 & 0.256 & 0.878 \\
\hline cy19:0 & 0.110 & 1.000 & 0.326 & 0.441 & -0.311 & 0.551 & -0.180 & 1.000 \\
\hline $20: 1 \omega 9$ & 0.052 & 1.000 & -0.163 & 1.000 & 0.523 & 0.012 & 0.526 & 0.013 \\
\hline $20: 2 \omega 6,9$ & -0.136 & 1.000 & -0.002 & 1.000 & -0.356 & 0.336 & 0.327 & 0.532 \\
\hline $20: 3 \omega 6$ & 0.343 & 0.379 & 0.071 & 1.000 & 0.712 & $<0.001$ & 0.277 & 0.820 \\
\hline 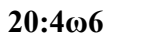 & -0.649 & $<0.001$ & 0.344 & 0.370 & 0.182 & 1.000 & 0.427 & 0.125 \\
\hline $20: 5 \omega 3$ & -0.696 & $<0.001$ & 0.562 & 0.004 & 0.168 & 1.000 & -0.393 & 0.224 \\
\hline
\end{tabular}

* Pearson correlation coefficient 


\section{Table S3.5}

ANOVA table of logit-transformed proportions for each fatty acid in the expanded 37-species dataset. Species and habitats are used as explanatory factors in the model. $P$-values were obtained after Holm's correction. Fatty acids significantly different between species are marked in bold.

\begin{tabular}{|c|c|c|c|c|c|c|c|c|c|}
\hline \multirow{2}{*}{ Fatty acid } & \multicolumn{3}{|c|}{ Habitat } & \multicolumn{3}{|c|}{ Species } & \multicolumn{3}{|c|}{ Habitat:Species } \\
\hline & df & F & Adjusted P & df & $F$ & Adjusted P & df & F & Adjusted P \\
\hline a15:0 & 2 & 3.090 & 0.327 & 36 & 0.877 & 1.000 & 9 & 0.415 & 1.000 \\
\hline i15:0 & 2 & 2.899 & 0.327 & 36 & 2.146 & 0.011 & 9 & 0.811 & 1.000 \\
\hline 16:0 & 2 & 23.017 & $<0.001$ & 36 & 3.557 & $<0.001$ & 9 & 1.065 & 1.000 \\
\hline 16:107 & 2 & 1.545 & 0.825 & 36 & 2.370 & 0.004 & 9 & 0.347 & 1.000 \\
\hline i16:0 & 2 & 3.160 & 0.327 & 36 & 1.422 & 0.440 & 9 & 0.192 & 1.000 \\
\hline i17:0 & 2 & 0.535 & 0.825 & 36 & 0.716 & 1.000 & 9 & 0.845 & 1.000 \\
\hline 18:0 & 2 & 15.104 & $<0.001$ & 36 & 2.619 & 0.001 & 9 & 3.424 & 0.016 \\
\hline $18: 1 \omega 7$ & 2 & 35.324 & $<0.001$ & 36 & 1.522 & 0.319 & 9 & 0.693 & 1.000 \\
\hline 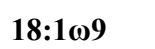 & 2 & 11.782 & $<0.001$ & 36 & 2.278 & 0.006 & 9 & 1.174 & 1.000 \\
\hline $18: 2 \omega 6,9$ & 2 & 31.610 & $<0.001$ & 36 & 3.176 & $<0.001$ & 9 & 0.918 & 1.000 \\
\hline cy19:0 & 2 & 12.049 & $<0.001$ & 36 & 1.379 & 0.440 & 9 & 1.191 & 1.000 \\
\hline 20:109 & 2 & 40.593 & $<0.001$ & 36 & 3.760 & $<0.001$ & 9 & 2.943 & 0.054 \\
\hline $20: 2 \omega 6,9$ & 2 & 1.603 & 0.825 & 36 & 0.841 & 1.000 & 9 & 0.103 & 1.000 \\
\hline $20: 3 \omega 6$ & 2 & 87.090 & $<0.001$ & 36 & 3.637 & $<0.001$ & 9 & 3.016 & 0.047 \\
\hline 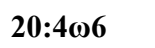 & 2 & 5.363 & 0.049 & 36 & 4.431 & $<0.001$ & 9 & 1.072 & 1.000 \\
\hline $20: 5 \omega 3$ & 2 & 1.244 & 0.825 & 36 & 3.418 & $<0.001$ & 9 & 1.961 & 0.671 \\
\hline
\end{tabular}




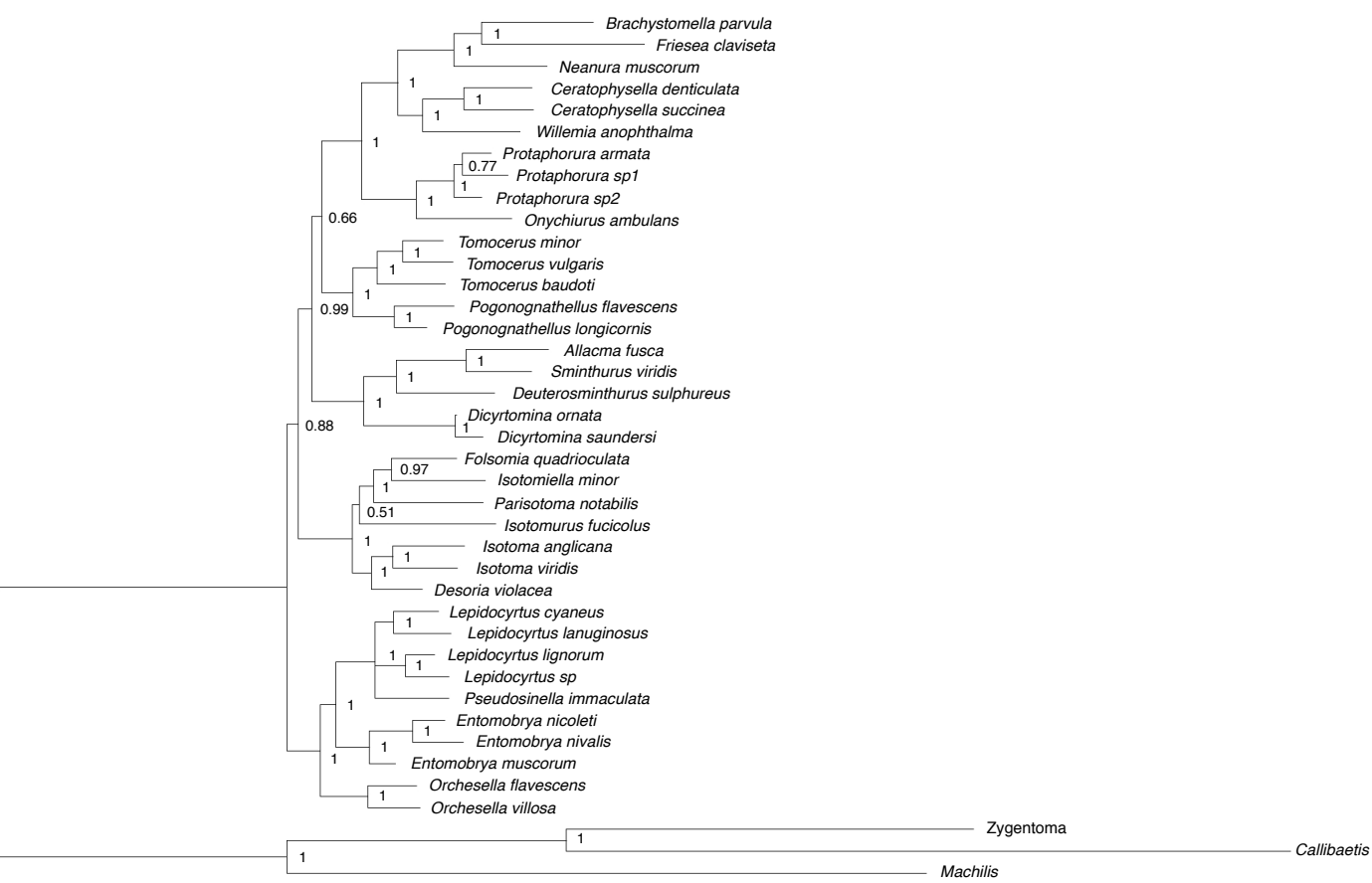

Figure S3.1

0.2

Bayesian Inference phylogeny of Collembola based on the concatenated DNA sequences of ribosomal 18S and $28 \mathrm{~S}$ rRNA and cytochrome oxidase I and Histone H3 genes. Callibaetis, Machilis and Zygentoma served as outgroups. Numbers at nodes represent Bayesian posterior probabilities.

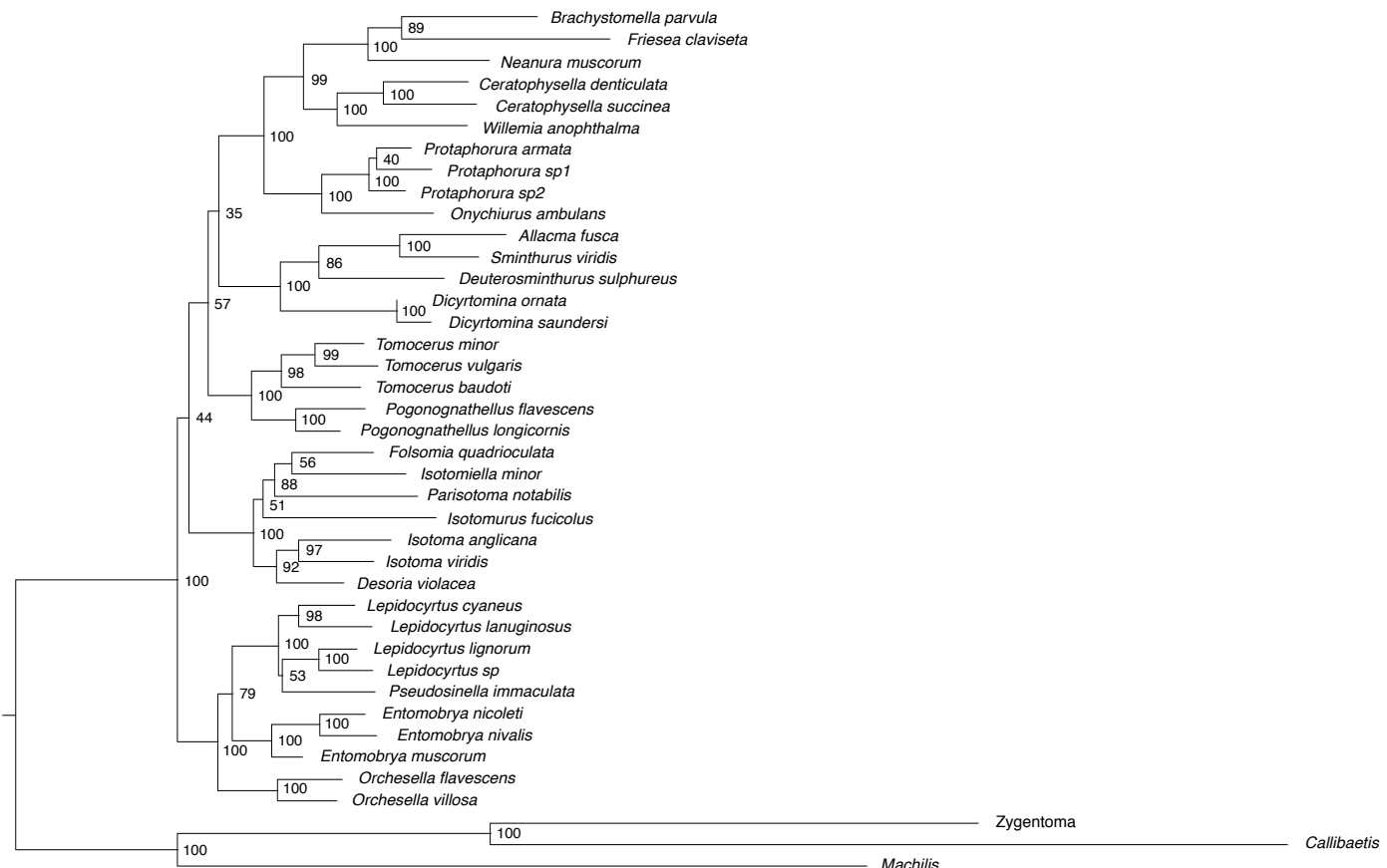

Figure S3.2

Maximum likelihood phylogeny of Collembola based on the concatenated DNA sequences of ribosomal $18 \mathrm{~S}$ and $28 \mathrm{~S}$ rRNA and cytochrome oxidase I and Histone H3 genes. Callibaetis, Machilis and Zygentoma served as outgroups. Numbers at nodes represent bootstrap values from Maximum Likelihood analyses. 


\title{
Chapter 4
}

\section{Mechanisms of Collembola species coexistence as indicated by phylogeny and functional traits}

Ting-Wen Chen, Jo-Fan Chao, Victoria Kreipe, Ina Schaefer, Matty P. Berg, Stefan Scheu

\begin{abstract}
The mechanisms driving and maintaining species coexistence in soil have long puzzled ecologists. To investigate the relative contributions to community assembly of different processes, such as environmental filtering and interspecific competition, community phylogenetic and trait-based approaches have recently been developed. If process-related traits exhibit phylogenetic signal, the presence of phylogenetically closely related species within local communities point to environmental filtering as the major structuring force, while low relatedness among coexisting species point to the dominance of biotic interactions or niche partitioning. In this study we inferred assembly processes of Collembola communities sampled from arable fields, grasslands and forests by exploring phylogenetic relatedness and functional trait similarities of local communities. We found associations between habitat types and Collembola phylogenetic group at the metacommunity scale. The results indicate that Collembola in arable fields were mainly structured by environmental filtering, while niche partitioning dominated in forests. Epedaphic (surface-living) species showed phylogenetic clustering in grasslands and forests, while in forests they also possessed similar traits. Hemiedaphic (sub-surface-dwelling) species in arable fields and grasslands were phylogenetically clustered, but in forests they were phylogenetically overdispersed with different traits. However, the assembly patterns of euedaphic (soil-dwelling) species in each of the three habitat types did not differ from random patterns. Furthermore, different phylogenetic (taxonomic) groups of Collembola showed different patterns in the three habitats. Overall, the results suggest that Collembola assemblages were driven by different mechanisms in the studied habitats, with the relative importance of these mechanisms varying between soil strata and between phylogenetic lineages. Future studies integrating phylogenetic comparative methods, trait-based approaches and community phylogeny will allow novel insight into assembly processes of soil communities.
\end{abstract}

\section{Keywords}

community assembly; co-occurrence; disturbance; environmental filtering; habitat; niche partitioning; soil; springtail; stochasticity; trait 


\section{Introduction}

Soils are among the most biodiverse ecosystems on earth and have been viewed as "the poor man's tropical rainforest" (Giller 1996). The coexistence of a multitude of animal species has puzzled soil ecologists for long, as reflected in the phrase "the enigma of soil animal species diversity" (Anderson 1975). How can so many species co-occur, and what are the mechanisms driving and maintaining species coexistence in local communities?

Community composition is influenced by a number of processes, including niche-related (Chase and Leibold 2003), neutral (Hubbell 2001) and biogeographical processes (Ricklefs 1987). Community phylogenetic framework (Webb et al. 2002, Cavender-Bares et al. 2009) and trait-based approaches (McGill et al. 2006; Adler et al. 2013) have been developed to investigate the relative importance of these processes for community assembly. In both frameworks, functional traits are crucial, since they form the mechanistic link between evolutionary processes and contemporary ecological processes (Cavender-Bares et al. 2009). On the one hand, traits are characters of species derived from their ancestors and thus, exhibit phylogenetic signal, i.e., phylogenetically related species possess similar traits. On the other hand, traits influence the performance and fitness of species in certain environments and are thus functional (Violle et al. 2007). If process-related traits exhibit phylogenetic signal, a community composed of phylogenetically closely related species is inferred to be structured by environmental filtering. In contrast, low relatedness among coexisting species points to the dominance of competitive interactions or niche partitioning (Webb et al. 2002, Cavender-Bares et al. 2009; but see Gerhold et al. 2015). Furthermore, using a phylogeny-weighted community matrix allows the measurement of phylogenetic dissimilarities between local communities and a trait-weighted matrix the measurement of trait dissimilarities (Pillar and Duarte, 2010). The phylogeny- or trait-weighted community matrix, combined with principle coordinate analysis, generates the principal coordinates of phylogenetic or trait structure of a metacommunity, allowing the identification of specific phylogenetic clades or species with certain traits that associate with habitats (Duarte, 2011, Duarte et al. 2016). In this study we adopted community phylogenetic approaches for the first time to investigate community assembly processes in one of the most abundant and diverse soil invertebrates, Collembola (springtails).

Collembola are basal Hexapoda that occur in high densities in soil with 10,000-100,000 individuals $\mathrm{m}^{-2}$ and local diversities of 60-80 species (Petersen and Luxton 1982). They occupy a wide variety of ecological niches, regulate soil microbial activity and contribute to decomposition processes and nutrient cycling (Petersen and Luxton, 1982, Rusek, 1998, Schaefer et al. 2009). Among the few soil taxa for which trait databases are available (Vandewalle et al. 2010, Pey et al. 2014, Moretti et al. 2017, Matty P. Berg, unpublished data), Collembola are ideal subjects for the application of trait-based and community phylogenetic approaches to investigate community assembly. The environmental associations of community structures and species functional traits suggest that both abiotic and biotic factors select for certain traits of Collembola (Ponge et al. 2003, Sousa et al. 2006, Makkonen et al. 2011, Bokhorst et al. 2012, Martins da Silva et al. 2012, Salmon and Ponge 2012, Heiniger et al. 2014, Widenfalk et al. 2015, 
Widenfalk et al. 2016, Pollierer and Scheu 2017). Spherical body shape, large body size, dark pigmentation and sexual reproduction are characteristics of species preferentially occurring in open habitats and at the soil surface, whereas small body size, lack of eye spots, pale color and asexual reproduction are typical traits of species in stable environment and in soil (Salmon et al. 2014). Furthermore, co-occurring Collembola species possessing similar traits suggest that disturbed habitats structure community assembly via abiotic factors (Widenfalk et al. 2015), while niche partitioning or interspecific competition are likely predominant processes in stable environments that result in co-occurring species with different traits (Widenfalk et al. 2016). Since the functional traits of Collembola derive from ancestors and thus exhibit phylogenetic signal (as being tested in Chapter 2), similar traits in local community also likely reflect close phylogenetic relationships between coexisting species.

In this study we explored habitat associations of Collembola phylogenetic groups and phylogenetic and trait patterns in local communities collected from arable fields, grasslands and forests, three types of habitats characterized by distinct disturbance regimes and dominating mosaic landscapes in Central Europe. We hypothesized that species co-occurring in heavily disturbed habitats, such as arable fields, show phylogenetic clustering and similar traits (Ding et al. 2012, Gianuca et al. 2014, Widenfalk et al. 2015), as disturbance functions as environmental filter selecting for specific traits that exhibit phylogenetic signal. In contrast, forests, representing stable habitats little disturbed by agricultural activities, allow complex soil food webs to be established (Scheu and Falca 2000, Digel et al. 2014), where different phylogenetic clades of Collembola occupy various niches and thus exhibit phylogenetic overdispersion with different traits in local communities. Since community phylogenetic analyses are sensitive to the spatial and taxonomical scales of the study (Cavender-Bares et al. 2006, Swenson et al. 2006), we further confined our analyses by defining species pools using habitat types (arable fields, grasslands and forests), vertical stratification of species (surface-living, sub-surface-dwelling and soildwelling species) and different phylogenetic (taxonomic) groups.

\section{Materials and Methods}

\section{Sampling}

Collembola were sampled between March and June 2014 from arable fields, grasslands and forests at six sites near Göttingen, Germany (Figure 2.1, Table S2.1, Chapter 2). In each of the arable fields and grasslands, one suction sample equal to a surface area of $154 \mathrm{~cm}^{2}$ was taken for $10 \mathrm{sec}$ to collect surfaceliving individuals. Then, to sample soil-dwelling individuals, a soil core sample $(5 \mathrm{~cm}$ diameter, $5 \mathrm{~cm}$ depth) was taken at the center of the area from which the suction sample was collected. In each forest, both litter and fragmented litter were collected by hand in an area of $154 \mathrm{~cm}^{2}$, followed by a $10 \mathrm{sec}$ suction sample of the humus layer. This suction sample was added to the litter collection producing a full litter-sample. Thereafter, a soil core sample was taken at the center of the same area previously sampled by hand-collection and suction. Collembola from the suction samples from arable fields and grasslands 
were directly transferred into $96 \%$ ethanol, while those in the litter and soil cores were extracted by heat using a Kempson extractor (Kempson et al. 1963), collected in water and then transferred into 96\% ethanol every two days over a period of ten days. Samples were kept at $4^{\circ} \mathrm{C}$ until identification and then stored at $-80^{\circ} \mathrm{C}$. Collembola identification was based on Hopkin (2007), Fjellberg $(1998,2007)$ and Gisin (1960). and the nomenclature followed Bellinger et al. (1996-2017; www.collembola.org). The density (individuals $\mathrm{m}^{-2}$ ) of each species in the suction (litter) sample was added to that in the soil samples for the following analyses.

\section{Phylogenetic and trait distances between species}

Phylogenetic relatedness and trait similarities of between species were calculated based on species pairwise distance matrices referred to their phylogeny and traits, respectively. The phylogenetic distance matrix was obtained from the ultrametric phylogenetic tree (Figure 2.3, Chapter 2) using the function cophenetic.phylo implemented in the R package "ape" (Paradis et al. 2004). The trait distance matrix was calculated based on species characters in body shape and length, pigmentation, number of ommatidia, vertical stratification and reproductive mode, multiple traits that exhibited phylogenetic signal (Chapter 2). While body length was used as a continuous variable, body shape, pigmentation, number of ommatidia, vertical stratification and reproductive mode were coded by binary variables for each state of traits. Gower's distances were calculated to generate a trait distance matrix using the function dist.ktab implemented in the R package "ade4” (Dray and Dufour 2007).

\section{Phylogenetic and trait structures of communities}

To investigate habitat associations with species phylogeny and traits, phylogeny- and trait-weighted community matrices were generated using a fuzzy-weighting method (Pillar and Duarte, 2010). Community data were first standardized using the "Hellinger" transformation (Legendre and Gallagher 2001) by the function decostand implemented in the R package "vegan" (Oksanen et al. 2015) and then weighted by species phylogenetic or trait distance metrics using the function matrix.p implemented in the R package "SYNCSA" (Debastiani and Pillar 2012). Differences in community phylogeny- or traitweighted compositions were calculated based on Bray-Curtis dissimilarity between communities, followed by distance-based multivariate analysis of variance (ADONIS) with 9,999 permutations using the function adonis implemented in the "vegan" package, to test habitat effects on community phylogeny- or trait-weighted compositions. If habitats had significant effects on community phylogeny- or traitweighted compositions, the weighted community compositions were applied to principle coordinate analysis (PCoA) using the function pcps implemented in the R package "PCPS" (Debastiani and Duarte 2014). This resulted in principal coordinates of phylogenetic structures (PCPS) and trait structures (PCTS) of the metacommunity. Pearson correlation coefficients of site scores and species densities at each PCPS axis were calculated using the function cor.test. $P$-values of correlation tests were adjusted using 
Benjamini and Hochberg corrections (BH; Benjamini and Hochberg 1995). Habitat effects on site scores of PCPS axes were further tested using Kruskal-Wallis rank sum test followed by Dunn's test with BH corrections for multiple comparisons.

\section{Phylogenetic relatedness and trait similarities of coexisting species}

To represent overall species relatedness of a local community, the Net Relatedness Index (NRI) was calculated based on mean pairwise distance (MPD) of a local community (Webb 2000). The NRI is a negative value of standardized effect size of MPD, for which the observed MPD was standardized by 999 simulated MPDs generated by drawing species with equal probability from the phylogenetic distance matrix ("phylogeny.pool" null model) representing the species pool, in which only species occurring in all the local communities were retained. A positive NRI indicates that the species in the observed community are more closely related than expected based on the null communities. A negative NRI, on the contrary, indicates that species within a local community were more distantly related to each other than expected. NRI was calculated using the function ses.mpd implemented in the R package "picante" (Kembel et al. 2010) for phylogenetic distance matrix or trait distance matrix using species presence/absence or density data.

Since considering different definitions of species pools may help to inspect assembly processes at different spatial or taxonomic levels (Emerson and Gillepsie 2008, Lessard et al. 2012), NRI was calculated based on the following definitions of species pools: (1) a total species pool considering all species recorded in the study (i.e., entire phylogenetic tree including all species); (2) habitat-specific species pools based on species occurrence in a certain habitat type: arable fields (37 species), grasslands (43 species) and forests (52 species; Figure S4.1). A significant pattern found in local communities after constraining the randomization process within a certain habitat-specific pool indicates that other factors beside the broad-defined habitat type influence community assembly at the local scale. These factors may relate to the microhabitat conditions, e.g. soil moisture, food resources, etc.; (3) soil horizontal species pools according to the data on vertical stratification of different species: epedaphic (surface-living, 19 species), hemiedaphic (sub-surface-dwelling, 30 species) and euedaphic (soil dwelling, 26 species; Figure S4.2). Species vertical stratification is relevant to the scale at which individuals likely interact; (4) different taxonomic (phylogenetic) scales at higher taxonomic levels including Entomobryomorpha (34 species), Poduromorpha (20 species) and Symphypleona (18 species), and at lower taxonomic levels for Entomobryoidea (14 species) and Isotomidae (17 species; Figure S4.3). Analyses across different taxonomic scales can help to inspect how evolution may have influenced contemporary species coexistence (Silver et al. 2012, Tanaka and Sato 2015), and assembly processes may differ between phylogenetic lineages (Ndiribe et al. 2013, Elliott et al. 2016). For each of the defined species pools, only the species belonging to the pool were retained in the community dataset as well as in the phylogenetic and trait distance matrices. 
Since the aim of the study was to infer assembly processes of Collembola in different habitats, the six sites were treated as replicates, and phylogenetic and trait NRI in each habitat was tested using Wilcoxon signed rank test against "0", which indicated a randomly assembled community. Kruskal-Wallis rank sum test was applied to compare NRI values among habitats.

\section{Results}

\section{Phylogenetic and trait structures between communities}

In total, 6,323 Collembola individuals were collected, with 75 morphologically defined species. Phylogeny-weighted community structures significantly differed among habitats $\left(\mathrm{R}^{2}=0.321, P=0.006\right.$, ADONIS). However, trait-weighted community structures were not different among habitats $\left(\mathrm{R}^{2}=0.292\right.$, $P=0.051$, ADONIS). Communities from forests were associated with Neelipleona and the clade comprising Poduromorpha and Symphypleona, with higher scores at PCPS 1 than that in grasslands and arable fields ( $P=0.019$, Kruskal-Wallis rank sum test), while at PCPS 2 communities in arable fields were associated with species of Isotomidae (Figure 4.1).

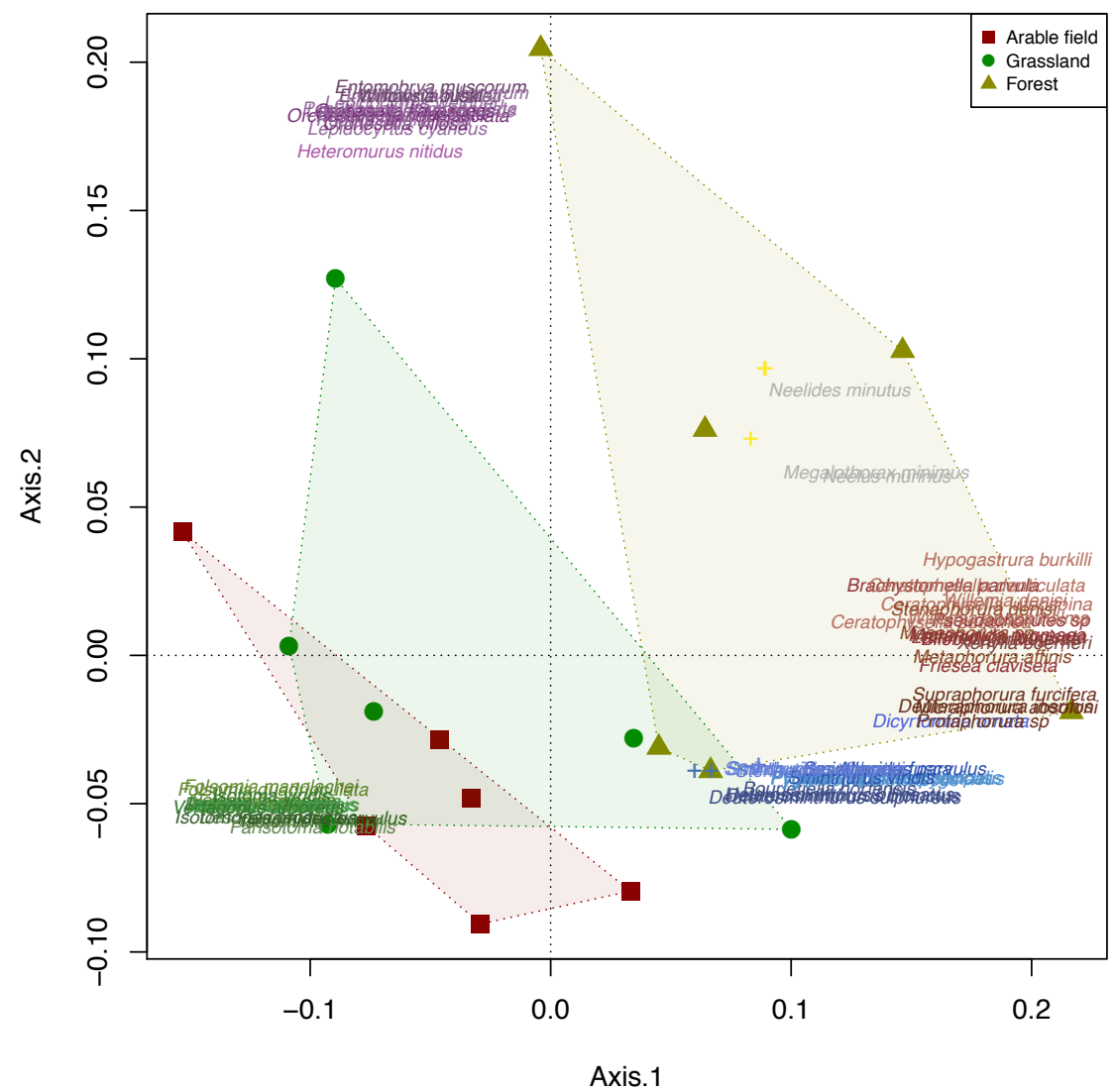

Figure 4.1

Scatter diagram of phylogenetic-weighted structure of Collembola communities (PCPS). Polygons encompass replicates of the same habitats. Only Collembola species significantly correlated to the axes are plotted with names and the others with cross symbols. 


\section{Phylogenetic relatedness and trait similarities of coexisting species}

When all the species were used as species pool, co-occurring Collembola showed phylogenetic overdispersion in forests (phylogenetic NRI $=-0.82 \pm 0.21$, mean $\pm \mathrm{SE} ; P=0.031$, Wilcoxon test). Mean phylogenetic relatedness of species inhabiting forests was significantly lower than those inhabiting arable fields and grasslands $(P=0.019$, Kruskal-Wallis test). Accounting for species density, communities in arable fields showed phylogenetic clustering (phylogenetic NRI $=1.68 \pm 0.37 ; P=0.031$, Wilcoxon test), indicating that abundant species in arable fields were closely related phylogenetically. Trait similarities in local communities, however, were not significantly different from random patterns in either of the three habitats, irrespective of using species presence/absence or density data (Table 4.1).

When the species pool was defined according to habitat types, similar patterns were detected to those using the entire species pool. In contrast, when the species pool was defined by vertical stratification of species, local communities exhibited different patterns of phylogenetic relatedness and trait similarity among different habitats. For epedaphic species, abundant species in grasslands and forests showed phylogenetic clustering, while forest species exhibited similar traits irrespective of whether species presence/absence or density data was considered. For hemiedaphic species, abundant species in arable fields and grasslands showed phylogenetic clustering, while species present in forests exhibited phylogenetic overdispersion and similar traits. Phylogenetic relatedness of the occurrence of hemiedaphic species was low in forests, intermediate in arable fields and high in grasslands. For euedaphic species, community phylogenetic relatedness and trait similarity in each of the three habitats were not different from random communities (Table 4.1).

At different taxonomic scales (phylogenetic clades), Collembola communities differed in their phylogenetic relatedness and trait similarities among the three habitats. At the order level, Entomobryomorpha, including Entomobryoidea, Tomoceridae and Isotomidae, showed similar patterns as compared to those using the entire species pool. When species densities were considered, weak but significantly similar traits were found in Symphypleona inhabiting arable fields, while phylogenetic overdispersion was detected in Poduromorpha living in forests. At the family level, Entomobryoidea in grasslands were from close relatives when species densities were considered, but displayed various traits when presence/absence data was used. In forests Entomobryoidea were phylogenetically clustered and carried similar traits when densities were considered. In contrast, Isotomidae in arable fields and grasslands showed phylogenetic overdispersion, while in forests they were phylogenetically closely related when densities were considered (Table 4.1, Figure S4.3).

\section{Discussion}

In this study we explored and compared phylogenetic and trait patterns of Collembola assemblages among arable fields, grasslands and forests, and inferred potential mechanisms driving Collembola 


\section{Table 4.1}

Phylogenetic and trait structures represented by Net Relatedness Index (NRI; mean \pm standard error), based on species occurrence (presence/absence, $\mathrm{p} / \mathrm{a}$ ) or density (abundance) of Collembola assemblages collected in different types of habitats. Different species pool definitions and taxonomic scales were applied. Numbers in parentheses indicate number of species in the species pool. A mean NRI value significantly different from zero $(P$ $<0.05$, Wilcoxon signed rank test) is marked in bold, with color in red indicating clustering and in blue overdispersion. Asterisks indicate NRI values significant different among habitats $(P<0.05$, Kruskal-Wallis rank sum test).

\begin{tabular}{|c|c|c|c|c|c|}
\hline \multirow{2}{*}{ Species pool definition } & \multirow{2}{*}{ Habitat } & \multicolumn{2}{|c|}{ Phylogenetic NRI } & \multicolumn{2}{|c|}{ Trait NRI } \\
\hline & & $\mathbf{p} / \mathbf{a}$ & Abundance & $\mathbf{p} / \mathbf{a}$ & Abundance \\
\hline \multirow[t]{3}{*}{ All species pool (75) } & Arable & $-0.04 \pm 0.27 *$ & $1.67 \pm 0.37$ & $0.74 \pm 0.51$ & $0.80 \pm 0.34$ \\
\hline & Grassland & $0.76 \pm 0.40 *$ & $1.13 \pm 0.51$ & $1.20 \pm 1.04$ & $0.94 \pm 0.84$ \\
\hline & Forest & $-0.82 \pm 0.21 *$ & $0.15 \pm 0.38$ & $-0.18 \pm 0.29$ & $0.22 \pm 0.22$ \\
\hline \multirow[t]{3}{*}{ Habitat pool } & Arable (37) & $-0.26 \pm 0.27 *$ & $1.42 \pm 0.34$ & $0.17 \pm 0.57$ & $0.61 \pm 0.34$ \\
\hline & Grassland (43) & $1.01 \pm 0.47 *$ & $1.14 \pm 0.47$ & $0.12 \pm 0.87$ & $0.44 \pm 0.69$ \\
\hline & Forest (52) & $-0.84 \pm 0.22 *$ & $0.17 \pm 0.38$ & $0.42 \pm 0.37$ & $0.41 \pm 0.24$ \\
\hline \multicolumn{6}{|l|}{ Vertical stratification pool } \\
\hline \multirow[t]{3}{*}{ Epedaphic (19) } & Arable & $0.03 \pm 0.39$ & $0.51 \pm 0.54$ & $-0.19 \pm 0.52$ & $-0.41 \pm 0.40 *$ \\
\hline & Grassland & $0.72 \pm 0.61$ & $1.09 \pm 0.25$ & $-0.07 \pm 0.39$ & $-0.75 \pm 0.48 *$ \\
\hline & Forest & $0.85 \pm 0.59$ & $1.40 \pm \mathbf{0 . 3 0}$ & $1.67 \pm \mathbf{0 . 5 2}$ & $1.88 \pm 0.17$ * \\
\hline \multirow[t]{3}{*}{ Hemiedaphic (30) } & Arable & $1.17 \pm 0.40$ * & $1.60 \pm 0.36$ & $-0.05 \pm 0.40$ & $-0.43 \pm 0.32$ \\
\hline & Grassland & $1.47 \pm 0.47 *$ & $1.64 \pm \mathbf{0 . 3 8}$ & $-0.36 \pm 0.62$ & $-0.36 \pm 0.55$ \\
\hline & Forest & $-0.88 \pm 0.18 *$ & $0.57 \pm 0.50$ & $-1.32 \pm 0.13$ & $-0.82 \pm 0.34$ \\
\hline \multirow[t]{3}{*}{ Euedaphic (26) } & Arable & $-0.51 \pm 0.19$ & $-0.52 \pm 0.27$ & $-0.18 \pm 0.31$ & $-0.07 \pm 0.28$ \\
\hline & Grassland & $-0.62 \pm 0.19$ & $-0.55 \pm 0.26$ & $0.02 \pm 0.24$ & $0.00 \pm 0.12$ \\
\hline & Forest & $0.28 \pm 0.39$ & $-0.18 \pm 0.26$ & $0.30 \pm 0.34$ & $0.71 \pm 0.35$ \\
\hline
\end{tabular}

\section{Taxonomic scale}

Order level

Entomobryomorpha (34)

Arable

Grassland

Forest

$\mathbf{0 . 8 0} \pm \mathbf{0 . 1 3}$

$-0.40 \pm 0.35$

$0.19 \pm 0.31$

$0.45 \pm 0.36$

$0.67 \pm 0.29$

$-0.27 \pm 0.43$

$0.38 \pm 0.41$

$\mathbf{- 0 . 4 8} \pm \mathbf{0 . 1 7}$

$0.12 \pm 0.24$

$0.32 \pm 0.53$

$-0.15 \pm 0.27$

Symphypleona (18)

Arable

Grassland

$-0.08 \pm 0.71$

$-0.06 \pm 0.76$

$0.80 \pm 0.35$

$0.63 \pm \mathbf{0 . 2 6}$

Forest

$-0.35 \pm 0.71$

$0.03 \pm 0.68$

$1.23 \pm 0.39$

$1.24 \pm 0.19$

$-0.38 \pm 0.22$

$-0.33 \pm 0.26$

$0.28 \pm 0.53$

$0.48 \pm 0.48$

Poduromorpha (20)

Arable

Grassland

$-0.24 \pm 0.50 \quad-0.16 \pm 0.39$

$0.56 \pm 0.72$

$0.64 \pm 0.57$

Forest

$-0.19 \pm 0.27$

$-0.32 \pm 0.34$

$-0.50 \pm 0.27$

$-\mathbf{0 . 5 7} \pm \mathbf{0 . 1 2}$

$-0.66 \pm 0.13$

$-0.60 \pm 0.25$

$-0.06 \pm 0.32$

$0.17 \pm 0.22$

Lower levels

Entomobryoidea (14)

Arable

Grassland

$1.03 \pm 0.97$

$1.05 \pm 0.83$

$-0.66 \pm 0.35 * \quad-0.61 \pm 0.30$ *

Forest

$1.66 \pm 0.75$

$1.58 \pm 0.31$

$\mathbf{- 0 . 6 8} \pm \mathbf{0 . 1 3}$ *

$-0.48 \pm 0.32 *$

$0.25 \pm 0.37$

$1.40 \pm 0.11$

$1.68 \pm 0.61 * 1.67 \pm \mathbf{0 . 1 7}$ *

Isotomidae (17)

Arable

$\mathbf{- 0 . 5 2} \pm \mathbf{0 . 0 7} *-0.46 \pm 0.21$ *

$0.47 \pm 0.62$

$0.40 \pm 0.33$

Grassland

$\mathbf{- 0 . 4 6} \pm \mathbf{0 . 1 5} * \quad-0.21 \pm 0.21$ *

$0.81 \pm 0.69$

$0.96 \pm 0.50$

Forest

$0.72 \pm 0.31 * 0.35 \pm \mathbf{0 . 0 6} *$

$-0.33 \pm 0.24$

$0.03 \pm 0.22$ 
assemblages using community phylogenetic approaches. We found strong phylogeny-habitat associations, with Isotomidae generally associated with arable fields and Neelipleona, Poduromorpha and Symphypleona with forests. Our results generally support the hypothesis that Collembola communities in disturbed habitats such as arable fields are mainly driven by environmental filtering and those in stable habitats such as forests are based on competitive interactions or niche partitioning.

\section{Phylogeny-habitat associations}

Phylogeny-weighted community structures of Collembola separated well based on the habitats that associated strongly with phylogenetic lineages. In the phylogenetic tree, Isotomidae and Entomobryoidea derived earlier than the other Collembola groups. Their associations with disturbed environments, here represented by arable fields and grasslands with fluctuating temperature and humidity, likely reflected their early terrestrialization history and exposure to harsh environments. Development of forest habitats later on was likely accompanied by the diversification of other Collembola groups such as Symphypleona and Poduromorpha. This indicates that different phylogenetic groups remained associated with certain types of habitats. However, as pointed out in Chapter 2, the phylogenetic relationships between Collembola orders remain to be resolved.

Since disturbance regimes differed between arable fields and forests, phylogeny-habitat associations were likely mediated by disturbance-tolerant traits and related to moisture and habitat preferences of species (Makkonen et al. 2011, Widenfalk et al. 2015). Presumably, species with close phylogenetic affinities share similar physiological traits reflecting adaptation to disturbance regimes. However, to prove this idea, physiological traits need to be measured directly and the phylogenetic signal of the traits needs to be tested.

\section{Community assembly in different habitats}

The low phylogenetic relatedness in disturbed habitats, represented by the arable fields in this study, is consistent with the low relatedness in other communities at high altitudes or in disturbed habitats (Ding et al. 2012, Pellissier et al. 2013, Gianuca et al. 2014). Harsh environments likely select for species with similar traits e.g. those for coping with stress (Widenfalk et al. 2015), and these traits are likely to exhibit phylogenetic signal (Silvertown et al. 2006). Although all the traits analyzed in this study exhibited phylogenetic signal (Chapter 2), phylogenetic clustering in communities inhabiting arable fields did not translate into trait similarity, i.e., not different from randomness, suggesting that while some of the examined traits were filtered by disturbance in arable fields, other traits included in this study likely differed among coexisting species and were presumably driven by niche partitioning. Traits exhibiting phylogenetic signal but not included in this study, e.g. those related to drought and heat tolerance (Dias et al. 2013, Chen et al. 2017, Chapter 3), might be more relevant to the environmental filtering process. 
In contrast, Collembola communities in forests were phylogenetically overdispersed, suggesting that assemblages of Collembola in stable habitats are based on competitive interactions, as argued in an earlier trait-based study on Collembola (Widenfalk et al. 2016). While little is known on the role of interspecific competition in community assembly of Collembola (Caruso et al. 2013), there is evidence that predatorprey interactions may regulate Collembola communities (Schneider and Maraun 2009, Birkhofer et al. 2010, Caruso et al. 2013). We therefore suggest that phylogenetic overdispersion and trait dissimilarity reflect resource-based niche partitioning. Stable habitats such as forests allow the establishment of complex soil food webs that include several trophic levels from primary decomposers to predators (Scheu and Falca 2000, Digel et al. 2014). Collembola species in forests span several trophic levels (Chahartaghi et al. 2005, Hishi et al. 2007, Pollierer et al. 2009, Hyodo et al. 2010) with taxonomically related species occupying similar trophic niches as represented by stable isotope signatures (Potapov et al. 2016). Phylogenetic overdispersion in forests therefore likely resulted from the presence of species affiliated to a variety of phylogenetic clades. This variety allows the community to cover a wide range of trophic niches and thereby completely exploit the available resources. Notably, when the species pool was defined based on the forest habitat, Collembola communities were still phylogenetically overdispersed in forests, suggesting that factors other than those associated with the broadly defined habitat, presumably local micro-habitat characteristics or food resources, influenced community assembly. Spatial heterogeneity across vertical soil layers in forests likely resulted in phylogenetic overdispersion (Berg et al. 1998, Berg and Bengtsson 2007). Sampling at a finer vertical spatial scale is needed to prove this idea.

Other scenarios also likely resulted in phylogenetic overdispersion in Collembola communities inhabiting forests, such as environmental filtering for phylogenetically convergent traits, a pattern in which distantly related species share similar traits (Emerson and Gillespie 2008). The morphological traits considered in this study, however, all exhibited phylogenetic signal and presumably more related to abiotic factors, pointing to the need to consider other types of traits such as those related to resource exploitation or interspecific competition. For soil animals, integrating stable isotope signatures, neutral lipid fatty acid composition and molecular gut content and microbiome analyses will shed new light on their trophic niches (Ferlian and Scheu 2014, Heidemann et al. 2014, Ferlian et al. 2015, Potapov et al. 2016, Chen et al. 2017, Gong et al. submitted), and these need to be considered as functional traits and included in future phylogenetic and trait-based analyses.

\section{Species pool definitions}

When the species pool was defined based on vertical stratification of species, a spatial scale where species are more likely to interact, the abundant epedaphic species in forests exhibited phylogenetic clustering with similar traits, suggesting that environmental filtering predominantly worked on the traits. For example, two congeneric species, Lepidocyrtus lanuginosus and L. lignorum, shared a number of traits and always coexisted in forests. In contrast, hemiedaphic Collembola in forests presumably were driven by niche partitioning that likely resulted in phylogenetic overdispersion. Species assigned to this category 
typically migrate between different soil layers and possess various traits. In deep soil (euedaphic communities), in contrast, community assembly was likely driven by stochastic processes or a balance between environmental filtering and niche partitioning, as indicated by randomness in both phylogenetic relatedness and trait similarity. However, in this study we used data on vertical stratification compiled from literature without proving if this also applied to the study sites. Further studies at a finer spatial scale of the vertical community composition along soil profiles are needed to explore coexistence patterns at the scale relevant to species interactions (Maaß et al. 2015).

Using different species pools defined by phylogenetic (taxonomic) groups may help to infer mechanisms driving communities across different hierarchical taxonomic groups and between different phylogenetic groups, although species may interact with each other irrespective of their phylogenetic affinities. Species of the same phylogenetic clade usually possess similar traits. Controlling the similarity at a higher level and then exploring patterns at a lower level may therefore reveal the patterns and processes working at the lower level, likely improving the detection of overdispersion (Swenson et al. 2006, Cavender-Bares et al. 2006, Elliott et al. 2016). Analyses confined to single phylogenetic clade in this study suggest that community assembly processes vary with taxonomic levels and between phylogenetic lineages. At the order level, phylogenetic and trait patterns in Entomobryomorpha resembled those if total Collembola was used as species pool. In contrast, community assembly processes in Symphypleona and Poduromorpha differed from those of total Collembola, with trait-based environmental filtering being important for Symphypleona in arable fields and niche partitioning for Poduromorpha in forests. At lower taxonomic level, phylogenetic clustering and similar traits of Entomobryoidea inhabiting forests point to the importance of environmental filtering working on the abundant species. In grasslands cooccurring Entomobryoidea species possessed divergent traits, including pigmentation and numbers of ommatidia, suggesting that niche partitioning played a major role. However, Entomobryoidea in grasslands exhibited phylogenetic clustering when species densities were accounted, presumably resulting from environmental filtering that selected other unmeasured traits. Interestingly, community assembly processes in Isotomidae differed from those of other Collembola taxa. Isotomidae inhabiting arable fields and grasslands were likely structured via niche partitioning, while environmental filtering dominated in Isotomidae inhabiting forests, the latter being most evident in Folsomia species.

\section{Integration of phylogenetic, trait and comparative approaches}

The community phylogenetic framework was proposed by plant ecologists 15 years ago and thereafter proven by its ability to infer assembly processes (Webb et al. 2002, Cavender-Bares et al. 2009, Vamosi et al. 2009). Application of this method to soil biota, however, just started recently and so far is restricted to fungi (Bässler et al. 2014, Thorn et al. 2016), nematodes (Li et al. 2014), termites (Hausberger and Korb 2015, Hausberger and Korb 2016) and beetles (Andújar et al. 2015, Thorn et al. 2016). Our study provides an example of integrating community phylogenetic and trait-based approaches in studies on the assembly processes of one of the most diverse soil microarthropod groups, Collembola. The 
phylogenetic signal first needs to be tested using comparative methods for process-related traits, such as Collembola body shape, body length, pigmentation, number of ommatidia, reproductive mode and vertical stratification that reflect species associations with the habitats (Salmon et al. 2014, Malcicka et al. 2017; Chapter 2). Significant differences in community phylogenetic relatedness then provide evidence on assembly processes, as shown in Collembola inhabiting arable fields and forests exhibiting different phylogenetic relatedness. In this approach phylogenetic information is used as a surrogate for functional similarity (Kembel 2009, Mouquet et al. 2012, Cadotte et al. 2013). However, other traits such as those related to dietary resources may be phylogenetically labile (Chen et al. 2017, Chapter 3) and therefore phylogeny may be of limited value as a proxy for traits. If these labile traits are at work, trait values of individual species need to be measured and trait-based approaches instead of community phylogeny need to be adopted. Nevertheless, since species phylogeny and traits are not mutually exclusive but complemented each other (Cadotte et al. 2013), integrating information on both functional traits and phylogenetic relationships promises major progress in understanding of assembly processes not only for aboveground biota but also for belowground organisms.

\section{Acknowledgements}

We thank Charlotte Seifert for her help in site selection. Special thanks to Guido Humpert for support during lab work. This study was supported by the German Ministry of Education, Science and Technology (BMBF) and by the Uyttenbogaard Eliasen Society via a personal grant to MPB. The authors have no conflict of interest to declare.

\section{Author Contributions}

TWC and SS conceived and designed the study; TWC, JFC, VK and MPB performed the study and collected the data; TWC analyzed the data; TWC, MPB, IS and SS wrote the manuscript.

\section{References}

Adler, P.B., Fajardo, A., Kleinhesselink, A.R. \& Kraft, N.J.B. (2013) Trait-based tests of coexistence mechanisms. Ecology Letters, 16, 1294-1306.

Anderson, J.M. (1975) The enigma of soil animal species diversity. Progress in Soil Zoology: Proceedings of the 5th International Colloquium on Soil Zoology (ed J. Vaněk), pp. 51-58. Springer Netherlands, Dordrecht.

Andújar, C., Arribas, P., Ruzicka, F., Platt, A.C., Timmermans, M.J.T.N. \& Vogler, A.P. (2015) Phylogenetic community ecology of soil biodiversity using mitochondrial metagenomics. Molecular Ecology, 24, 3603-3617.

Bässler, C., Ernst, R., Cadotte, M., Heibl, C. \& Müller, J. (2014) Near-to-nature logging influences fungal community assembly processes in a temperate forest (ed J Barlow). Journal of Applied Ecology, 51, 939-948.

Bellinger, P.F., Christiansen, K.A. \& Janssens, F. Checklist of the Collembola of the world (www.collembola.org). 
Benjamini, Y. \& Hochberg, Y. (1995) Controlling the false discovery rate: a practical and powerful approach to multiple testing. Journal of the Royal Statistical Society Series B, 57, 289-300.

Berg, M.P. \& Bengtsson, J. (2007) Temporal and spatial variability in soil food web structure. Oikos, 116, $1789-1804$.

Berg, M.P., Kniese, J.P., Bedaux, J.J.M. \& Verhoef, H.A. (1998) Dynamics and stratification of functional groups of micro- and mesoarthropods in the organic layer of a Scots pine forest. Biology and Fertility of Soils, 26, $268-284$.

Birkhofer, K., Scheu, S. \& Wiegand, T. (2010) Assessing spatiotemporal predator-prey patterns in heterogeneous habitats. Basic and Applied Ecology, 11, 486-494.

Bokhorst, S., Phoenix, G.K., Bjerke, J.W., Callaghan, T. V., Huyer-Brugman, F. \& Berg, M.P. (2012) Extreme winter warming events more negatively impact small rather than large soil fauna: shift in community composition explained by traits not taxa. Global Change Biology, 18, 1152-1162.

Cadotte, M., Albert, C.H. \& Walker, S.C. (2013) The ecology of differences: assessing community assembly with trait and evolutionary distances. Ecology Letters, 16, 1234-1244.

Caruso, T., Trokhymets, V., Bargagli, R. \& Convey, P. (2013) Biotic interactions as a structuring force in soil communities: evidence from the micro-arthropods of an Antarctic moss model system. Oecologia, 172, 495-503.

Cavender-Bares, J., Keen, A. \& Miles, B. (2006) Phylogenetic structure of Floridian plant communities depends on taxonomic and spatial scale. Ecology, 87, S109-22.

Cavender-Bares, J., Kozak, K.H., Fine, P. V \& Kembel, S.W. (2009) The merging of community ecology and phylogenetic biology. Ecology Letters, 12, 693-715.

Chahartaghi, M., Langel, R., Scheu, S. \& Ruess, L. (2005) Feeding guilds in Collembola based on nitrogen stable isotope ratios. Soil Biology and Biochemistry, 37, 1718-1725.

Chase, J.M. \& Leibold, M.A. (2003) Ecological Niches: Linking Classical and Contemporary Approaches. University of Chicago Press, Chicago, IL.

Chen, T.-W., Sandmann, P., Schaefer, I. \& Scheu, S. (2017) Neutral lipid fatty acid composition as trait and constraint in Collembola evolution. Ecology and Evolution, 7, 9624-9638.

Debastiani, V.J. \& Duarte, L.D.S. (2014) PCPS - an R-package for exploring phylogenetic eigenvectors across metacommunities. Frontiers of Biogeography, 6, 144-148.

Debastiani, V.J. \& Pillar, V.D. (2012) SYNCSA--R tool for analysis of metacommunities based on functional traits and phylogeny of the community components. Bioinformatics, 28, 2067-8.

Dias, A.T.C., Krab, E.J., Mariën, J., Zimmer, M., Cornelissen, J.H.C., Ellers, J., Wardle, D.A. \& Berg, M.P. (2013) Traits underpinning desiccation resistance explain distribution patterns of terrestrial isopods. Oecologia, 172, 667677.

Digel, C., Curtsdotter, A., Riede, J., Klarner, B. \& Brose, U. (2014) Unravelling the complex structure of forest soil food webs: higher omnivory and more trophic levels. Oikos, 123, 1157-1172.

Ding, Y., Zang, R., Letcher, S.G., Liu, S. \& He, F. (2012) Disturbance regime changes the trait distribution, phylogenetic structure and community assembly of tropical rain forests. Oikos, 121, 1263-1270.

Dray, S. \& Dufour, A.-B. (2007) The ade4 Package: Implementing the duality diagram for ecologists. Journal of Statistical Software, 22.

Duarte, L.D.S. (2011) Phylogenetic habitat filtering influences forest nucleation in grasslands. Oikos, 120, $208-215$.

Duarte, L.D.S., Debastiani, V.J., Freitas, A.V.L. \& Pillar, V.D. (2016) Dissecting phylogenetic fuzzy weighting: theory and application in metacommunity phylogenetics. Methods in Ecology and Evolution, 7, 937-946.

Elliott, T.L., Waterway, M.J. \& Davies, T.J. (2016) Contrasting lineage-specific patterns conceal community phylogenetic structure in larger clades. Journal of Vegetation Science, 27, 69-79. 
Emerson, B.C. \& Gillespie, R.G. (2008) Phylogenetic analysis of community assembly and structure over space and time. Trends in Ecology \& Evolution, 23, 619-630.

Ferlian, O., Klarner, B., Langeneckert, A.E. \& Scheu, S. (2015) Trophic niche differentiation and utilisation of food resources in collembolans based on complementary analyses of fatty acids and stable isotopes. Soil Biology and Biochemistry, 82, 28-35.

Ferlian, O. \& Scheu, S. (2014) Shifts in trophic interactions with forest type in soil generalist predators as indicated by complementary analyses of fatty acids and stable isotopes. Oikos, 123, 1182-1191.

Fjellberg, A. (1998) The Collembola of Fennoscandia and Denmark Part I: Poduromorpha (eds NP Kristensen and V Michelsen). Koninklijke Brill, Leiden. The Netherlands.

Fjellberg, A. (2007) The Collembola of Fennoscandia and Denmark Part II: Entomobryomorpha and Symphypleona (eds NP Kristensen and V Michelsen). Koninklijke Brill, Leiden. The Netherlands.

Gerhold, P., Cahill, J.F., Winter, M., Bartish, I. V \& Prinzing, A. (2015) Phylogenetic patterns are not proxies of community assembly mechanisms (they are far better). Functional Ecology, 29, 600-614.

Gianuca, A.T., Dias, R.A., Debastiani, V.J. \& Duarte, L.D.S. (2014) Habitat filtering influences the phylogenetic structure of avian communities across a coastal gradient in southern Brazil. Austral Ecology, 39, 29-38.

Giller, P.S. (1996) The diversity of soil communities, the 'poor man's tropical rainforest'. Biodiversity and Conservation, 5, 135-168.

Gisin, H. (1960) Collembolenfauna Europas. Muséum d'Histoire Naturelle, Geneva.

Gong, X., Chen, T.-W., Zieger, S., Bluhm, C., Heidemann, K., Schaefer, I., Maraun, M., Liu, M. \& Scheu, S. Phylogenetic and trophic determinants of gut microbiota in soil detritivores. Submitted.

Hausberger, B. \& Korb, J. (2015) A phylogenetic community approach for studying termite communities in a West African savannah. Biology Letters, 11, 20150625.

Hausberger, B. \& Korb, J. (2016) The impact of anthropogenic disturbance on assembly patterns of termite communities. Biotropica, 48, 356-364.

Heidemann, K., Hennies, A., Schakowske, J., Blumenberg, L., Ruess, L., Scheu, S. \& Maraun, M. (2014) Free-living nematodes as prey for higher trophic levels of forest soil food webs. Oikos, 123, 1199-1211.

Heiniger, C., Barot, S., Ponge, J.-F., Salmon, S., Botton-Divet, L., Carmignac, D. \& Dubs, F. (2014) Effect of habitat spatiotemporal structure on collembolan diversity. Pedobiologia, 57, 103-117.

Hishi, T., Hyodo, F., Saitoh, S. \& Takeda, H. (2007) The feeding habits of collembola along decomposition gradients using stable carbon and nitrogen isotope analyses. Soil Biology and Biochemistry, 39, 1820-1823.

Hopkin, S.P. (2007) A Key to the Collembola (Springtails) of Britain and Ireland (ed Field Studies Council (FSC)). Shrewsbury.

Hubbell, S.P. (2001) The Unified Neutral Theory of Biodiversity and Biogeography. Princeton University Press, Princeton and Oxford.

Hyodo, F., Matsumoto, T., Takematsu, Y., Kamoi, T., Fukuda, D., Nakagawa, M. \& Itioka, T. (2010) The structure of a food web in a tropical rain forest in Malaysia based on carbon and nitrogen stable isotope ratios. Journal of Tropical Ecology, 26, 205-214.

Kembel, S.W. (2009) Disentangling niche and neutral influences on community assembly: assessing the performance of community phylogenetic structure tests. Ecology letters, 12, 949-60.

Kembel, S.W., Cowan, P.D., Helmus, M.R., Cornwell, W.K., Morlon, H., Ackerly, D.D., Blomberg, S.P. \& Webb, C.O. (2010) Picante: R tools for integrating phylogenies and ecology. Bioinformatics, 26, 1463-4.

Kempson, D., Lloyd, M. \& Ghelardi, R. (1963) A new extractor for woodland litter. Pedobiologia, 3, 1-21. 
Legendre, P. \& Gallagher, E.D. (2001) Ecologically meaningful transformations for ordination of species data. Oecologia, 129, 271-280.

Lessard, J.-P., Belmaker, J., Myers, J.A., Chase, J.M. \& Rahbek, C. (2012) Inferring local ecological processes amid species pool influences. Trends in Ecology \& Evolution, 27, 600-607.

Li, J., Li, S., Chen, Y., Jia, P., Hua, Z., Wang, S., Song, Y., Liao, B. \& Shu, W. (2014) Phylogenetic structures of soil nematode communities along a successional gradient in an unreclaimed copper mine tailings site. Soil Biology and Biochemistry, 77, 179-186.

Maaß, S., Maraun, M., Scheu, S., Rillig, M.C. \& Caruso, T. (2015) Environmental filtering vs. resource-based niche partitioning in diverse soil animal assemblages. Soil Biology and Biochemistry, 85, 145-152.

Makkonen, M., Berg, M.P., van Hal, J.R., Callaghan, T. V., Press, M.C. \& Aerts, R. (2011) Traits explain the responses of a sub-arctic Collembola community to climate manipulation. Soil Biology and Biochemistry, 43, 377-384.

Malcicka, M., Berg, M.P. \& Ellers, J. (2017) Ecomorphological adaptations in Collembola in relation to feeding strategies and microhabitat. European Journal of Soil Biology, 78, 82-91.

Martins da Silva, P., Berg, M.P., Serrano, A.R.M., Dubs, F. \& Sousa, J.P. (2012) Environmental factors at different spatial scales governing soil fauna community patterns in fragmented forests. Landscape Ecology, 27, 1337-1349.

McGill, B.J., Enquist, B.J., Weiher, E. \& Westoby, M. (2006) Rebuilding community ecology from functional traits. Trends in Ecology \& Evolution, 21, 178-185.

Moretti, M., Dias, A.T.C., de Bello, F., Altermatt, F., Chown, S.L., Azcarate, F.M., Bell, J.R., Fournier, B., Hedde, M., Hortal, J., Ibanez, S., Öckinger, E., Sousa, J.P., Ellers, J. \& Berg, M.P. (2017) Handbook of protocols for standardized measurement of terrestrial invertebrate functional traits. Functional Ecology, 31, 558-567.

Mouquet, N., Devictor, V., Meynard, C.N., Munoz, F., Bersier, L.F., Chave, J., Couteron, P., Dalecky, A., Fontaine, C., Gravel, D., Hardy, O.J., Jabot, F., Lavergne, S., Leibold, M.A., Mouillot, D., Munkemuller, T., Pavoine, S., Prinzing, A., Rodrigues, A.S., Rohr, R.P., Thebault, E. \& Thuiller, W. (2012) Ecophylogenetics: advances and perspectives. Biological Reviews, 87, 769-785.

Ndiribe, C., Pellissier, L., Antonelli, S., Dubuis, A., Pottier, J., Vittoz, P., Guisan, A. \& Salamin, N. (2013) Phylogenetic plant community structure along elevation is lineage specific. Ecology and Evolution, 3, 4925-4939.

Oksanen, J., Blanchet, F.G., Kindt, R., Legendre, P., Minchin, P.R., O’Hara, R.B., Simpson, G.L., Solymos, P., Stevens, M.H.H. \& Wagner, H. (2015) vegan: community ecology package.

Paradis, E., Claude, J. \& Strimmer, K. (2004) APE: Analyses of phylogenetics and evolution in R language. Bioinformatics, 20, 289-290.

Pellissier, L., Pradervand, J.-N., Williams, P.H., Litsios, G., Cherix, D. \& Guisan, A. (2013) Phylogenetic relatedness and proboscis length contribute to structuring bumblebee communities in the extremes of abiotic and biotic gradients (ed A Baselga). Global Ecology and Biogeography, 22, 577-585.

Petersen, H. \& Luxton, M. (1982) A comparative analysis of soil fauna populations and their role in decomposition processes. Oikos, 39, 288-388.

Pey, B., Nahmani, J., Auclerc, A., Capowiez, Y., Cluzeau, D., Cortet, J., Decaëns, T., Deharveng, L., Dubs, F., Joimel, S., Briard, C., Grumiaux, F., Laporte, M.-A.A., Pasquet, A., Pelosi, C., Pernin, C., Ponge, J.-F., Salmon, S., Santorufo, L. \& Hedde, M. (2014) Current use of and future needs for soil invertebrate functional traits in community ecology. Basic and Applied Ecology, 15, 194-206.

Pillar, V.D. \& Duarte, L.D.S. (2010) A framework for metacommunity analysis of phylogenetic structure. Ecology Letters, 13, 587-596.

Pollierer, M.M., Langel, R., Scheu, S. \& Maraun, M. (2009) Compartmentalization of the soil animal food web as indicated by dual analysis of stable isotope ratios $\left({ }^{15} \mathrm{~N} /{ }^{14} \mathrm{~N}\right.$ and $\left.{ }^{13} \mathrm{C} /{ }^{12} \mathrm{C}\right)$. Soil Biology and Biochemistry, 41, 12211226. 
Pollierer, M.M. \& Scheu, S. (2017) Driving factors and temporal fluctuation of Collembola communities and reproductive mode across forest types and regions. Ecology and Evolution, ece3.3035.

Ponge, J.-F., Gillet, S., Dubs, F., Fedoroff, E., Haese, L., Sousa, J.P. \& Lavelle, P. (2003) Collembolan communities as bioindicators of land use intensification. Soil Biology and Biochemistry, 35, 813-826.

Potapov, A.A., Semenina, E.E., Korotkevich, A.Y., Kuznetsova, N.A. \& Tiunov, A. V. (2016) Connecting taxonomy and ecology: Trophic niches of collembolans as related to taxonomic identity and life forms. Soil Biology and Biochemistry, 101, 20-31.

Ricklefs, R.E. (1987) Community diversity: Relative roles of local and regional processes. Science, 235, 167-171.

Rusek, J. (1998) Biodiversity of Collembola and their functional role in the ecosystem. Biodiversity and Conservation, 7 , 1207-1219.

Salmon, S. \& Ponge, J.-F. (2012) Species traits and habitats in springtail communities: A regional scale study. Pedobiologia, 55, 295-301.

Salmon, S., Ponge, J.-F., Gachet, S., Deharveng, L., Lefebvre, N. \& Delabrosse, F. (2014) Linking species, traits and habitat characteristics of Collembola at European scale. Soil Biology and Biochemistry, 75, 73-85.

Schaefer, M., Migge-Kleian, S. \& Scheu, S. (2009) The role of soil fauna for decomposition of plant residues. Functioning and Management of European Beech Ecosystems (eds R. Brumme \& P.K. Khanna), pp. 207-230. Springer Berlin Heidelberg, Berlin, Heidelberg.

Scheu, S. \& Falca, M. (2000) The soil food web of two beech forests (Fagus sylvatica) of contrasting humus type: stable isotope analysis of a macro- and a mesofauna-dominated community. Oecologia, 123, 285-296.

Schneider, K. \& Maraun, M. (2009) Top-down control of soil microarthropods - Evidence from a laboratory experiment. Soil Biology and Biochemistry, 41, 170-175.

Silver, C.A., Vamosi, S.M. \& Bayley, S.E. (2012) Temporary and permanent wetland macroinvertebrate communities: Phylogenetic structure through time. Acta Oecologica, 39, 1-10.

Silvertown, J., McConway, K., Gowing, D., Dodd, M., Fay, M.F., Joseph, J.A. \& Dolphin, K. (2006) Absence of phylogenetic signal in the niche structure of meadow plant communities. Proceedings of the Royal Society B, 273, 3944.

Sousa, J.P., Bolger, T., da Gama, M.M., Lukkari, T., Ponge, J.-F., Simón, C., Traser, G., Vanbergen, A.J., Brennan, A., Dubs, F., Ivitis, E., Keating, A., Stofer, S. \& Watt, A.D. (2006) Changes in Collembola richness and diversity along a gradient of land-use intensity: A pan European study. Pedobiologia, 50, 147-156.

Swenson, N.G., Enquist, B.J., Pither, J., Thompson, J. \& Zimmerman, J.K. (2006) The problem and promise of scale dependency in community phylogenetics. Ecology, 87, 2418-2424.

Tanaka, T. \& Sato, T. (2015) Taxonomic, phylogenetic and functional diversities of ferns and lycophytes along an elevational gradient depend on taxonomic scales. Plant Ecology, 216, 1597-1609.

Thorn, S., Bässler, C., Bernhardt-Römermann, M., Cadotte, M., Heibl, C., Schäfer, H., Seibold, S. \& Müller, J. (2016) Changes in the dominant assembly mechanism drives species loss caused by declining resources. Ecology Letters, 19, 163-170.

Vamosi, S.M., Heard, S.B., Vamosi, J.C. \& Webb, C.O. (2009) Emerging patterns in the comparative analysis of phylogenetic community structure. Molecular Ecology, 18, 572-592.

Vandewalle, M., de Bello, F., Berg, M.P., Bolger, T., Dolédec, S., Dubs, F., Feld, C.K., Harrington, R., Harrison, P. A., Lavorel, S., Silva, P.M., Moretti, M., Niemelä, J., Santos, P., Sattler, T., Sousa, J.P., Sykes, M.T., Vanbergen, A.J. \& Woodcock, B.A. (2010) Functional traits as indicators of biodiversity response to land use changes across ecosystems and organisms. Biodiversity and Conservation, 19, 2921-2947.

Violle, C., Navas, M.L., Vile, D., Kazakou, E., Fortunel, C., Hummel, I. \& Garnier, E. (2007) Let the concept of trait be functional! Oikos, 116, 882-892. 
Webb, C.O. (2000) Exploring the Phylogenetic Structure of Ecological Communities: An Example for Rain Forest Trees. The American Naturalist, 156, 145-155.

Webb, C.O., Ackerly, D.D., McPeek, M.A. \& Donoghue, M.J. (2002) Phylogenies and community ecology. Annual Review of Ecology and Systematics, 33, 475-505.

Widenfalk, L.A., Bengtsson, J., Berggren, A., Zwiggelaar, K., Spijkman, E., Huyer-Brugman, F. \& Berg, M.P. (2015) Spatially structured environmental filtering of collembolan traits in late successional salt marsh vegetation. Oecologia, 179, 537-549.

Widenfalk, L.A., Malmström, A., Berg, M.P. \& Bengtsson, J. (2016) Small-scale Collembola community composition in a pine forest soil - Overdispersion in functional traits indicates the importance of species interactions. Soil Biology and Biochemistry, 103, 52-62. 


\section{Supplementary Materials}
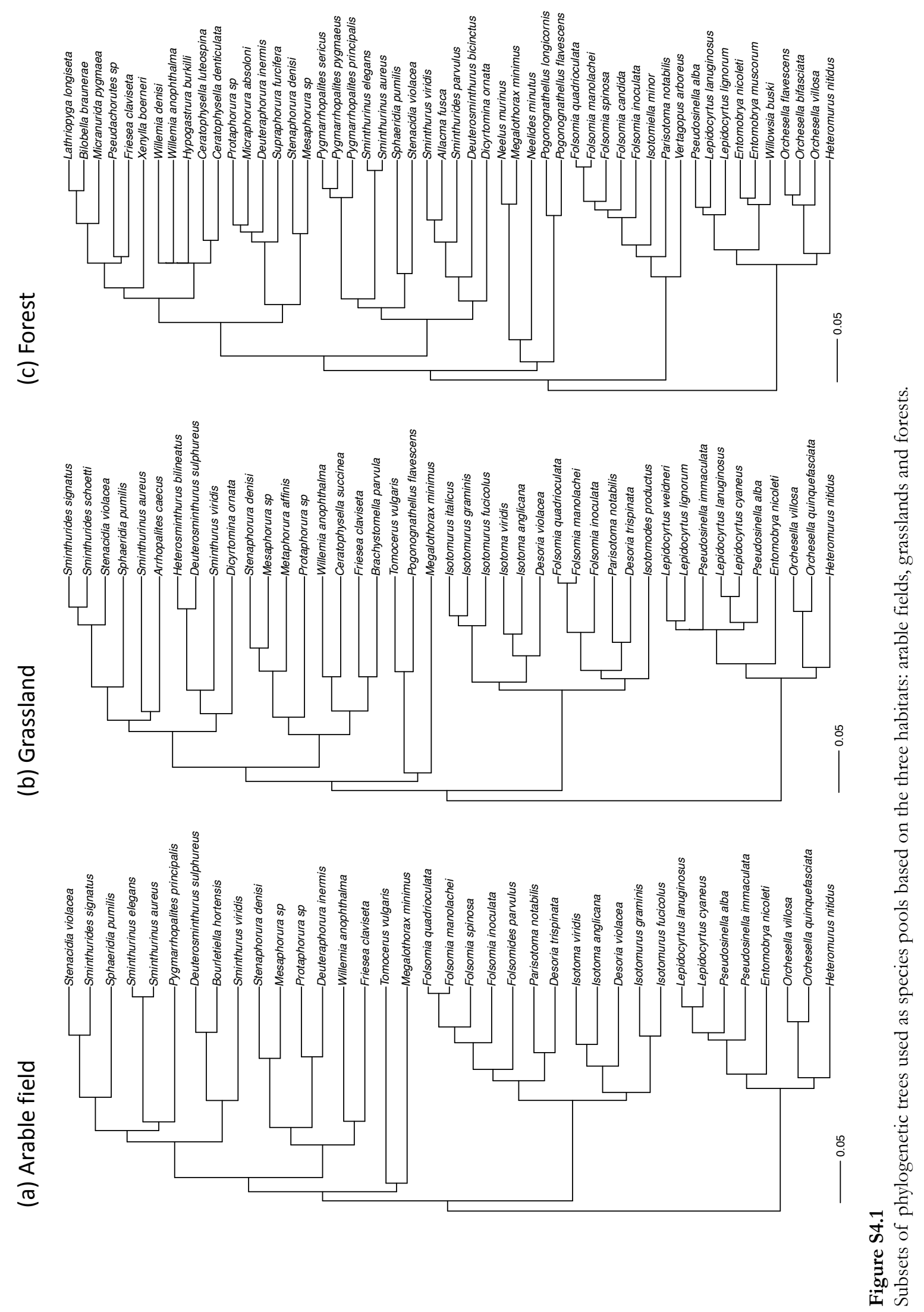


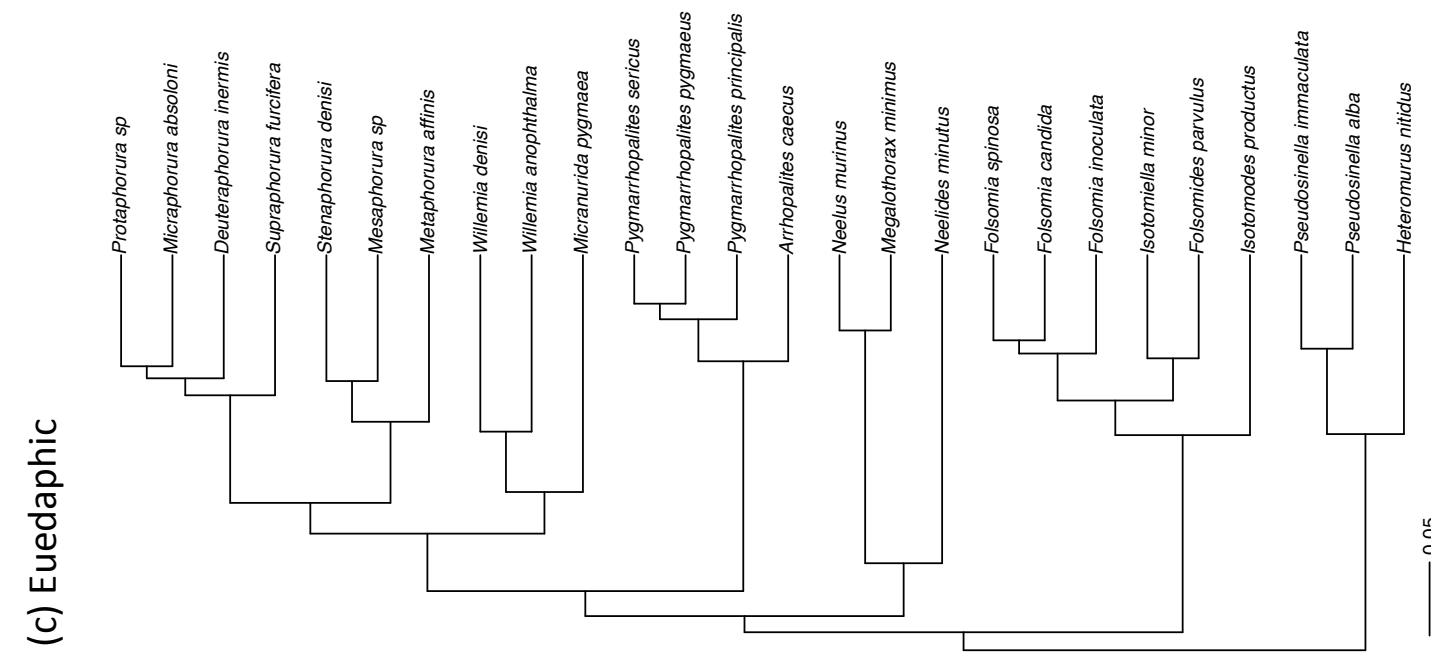

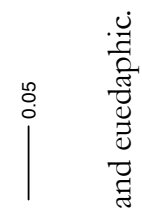

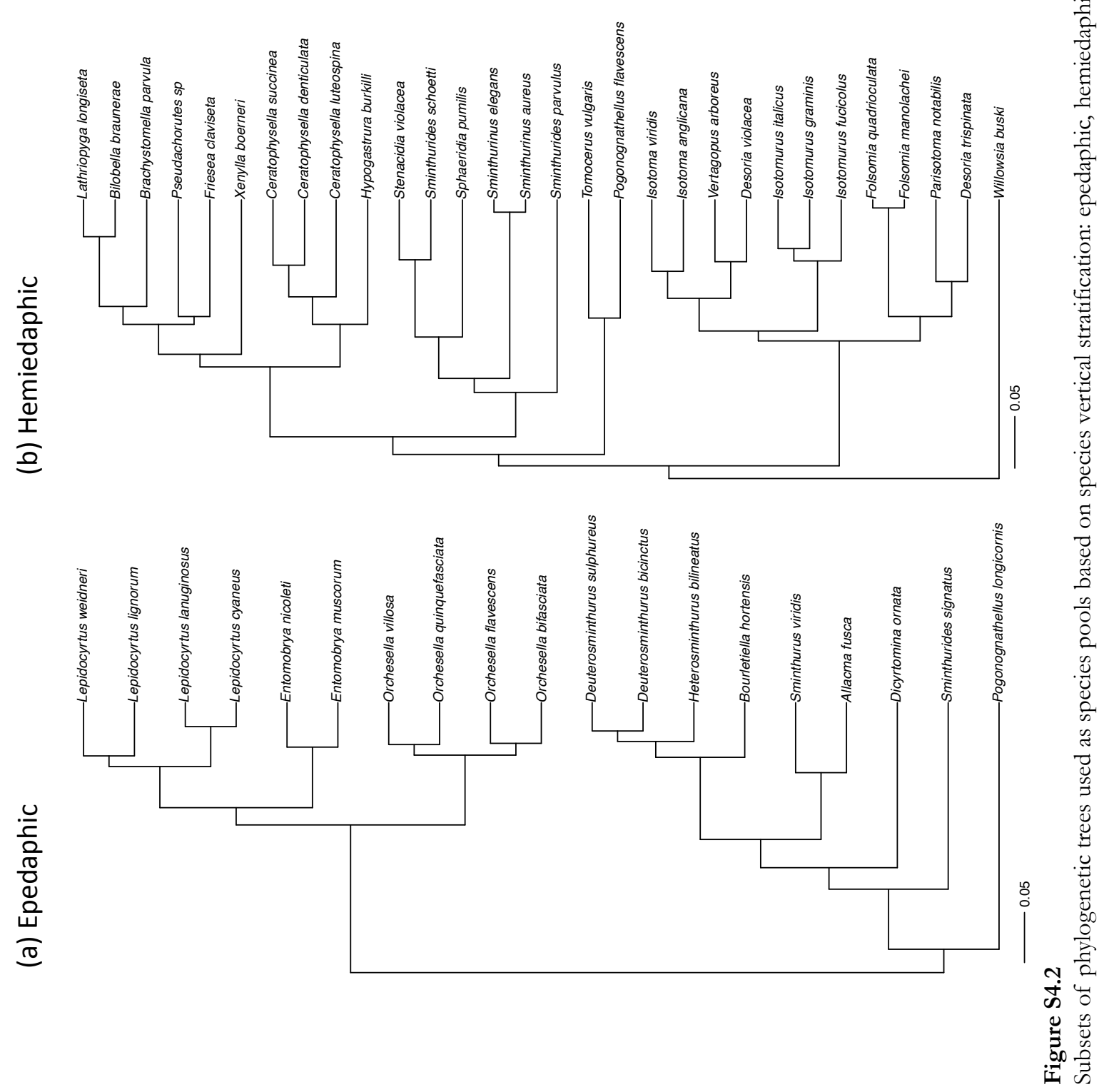




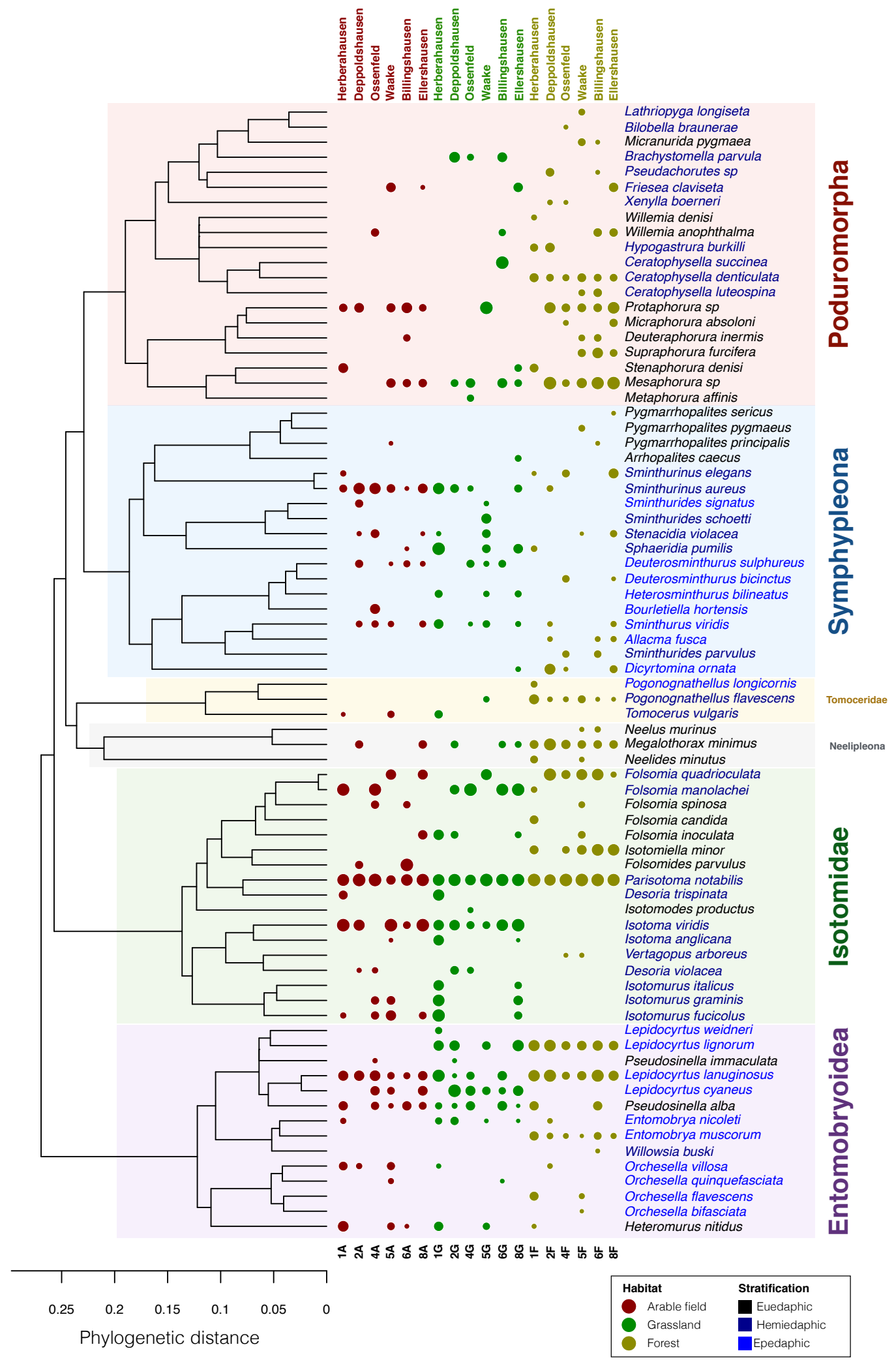

Figure S4.3

Densities of Collembola collected from the study sites plotted against the ultrametric phylogenetic tree. Size of circles represents standardized relative density of a species in a given community. Color of circles represent habitat type a species was sampled. Colors in the species names indicate vertical stratification of species. See Figure 2.4 and Chapter 2 for details on phylogenetic reconstruction of Collembola. 


\title{
Chapter 5
}

\section{Inferring assembly processes of Collembola communities along successional trajectories using phylogenetic approaches}

Ting-Wen Chen, Gerrit Moser, Apolline Auclerc, Jean-François Ponge, Sébastien Barot, Florence Dubs, Stefan Scheu

\begin{abstract}
Understanding ecological and evolutionary processes in community assembly can help to explain species coexistence of soil biota. Along successional trajectories, dispersal of species interacting with selection processes determines species composition in local communities. Intermediate dispersal with local environmental selection results in "species sorting" according to metacommunity theory, while frequent dispersal leads to "mass effect". As a consequence, the community may show a random pattern in early stages of succession but may follow deterministic pathways in later stages. In this study, we applied community phylogenetic approaches to a manipulative soil block experiment (Auclerc et al. 2009; Soil Biology and Biochemistry 41, 1596-1604), to infer factors influencing community assembly of soil springtails (Hexapoda: Collembola) during succession in forest and meadow soil. Soil blocks were defaunated and/or exchanged, and Collembola colonization from surrounding habitat into soil blocks was monitored one week, one month and six months after soil block reinstallation to the fields. We found phylogenetic signal of species preference to the soil types and of their dispersal ability in meadow but not in forest. Community assembly processes in meadow were predominated by niche partitioning, while in forest environmental filtering was the main driver. While environmental filtering continuously influenced Collembola community assembly in forest during the whole succession period, niche partitioning worked during Collembola succession in meadow and was significant at the later successional stage. Our results also indicate that soil properties of the defaunated and transferred blocks influenced community assembly of Collembola at initial stages of succession, in line with the species sorting scenario of metacommunity theory, while at later stages community assembly was dominated by mass effect. Furthermore, indigenous Collembola species survived in forest soil blocks that were transferred to the meadow habitat. In contrast, while most indigenous meadow species transferred to forest did not survive, Isotomidae species survived in meadow soil blocks and likely also immigrated from the surrounding forest habitat.
\end{abstract}

\section{Keywords}

ancestral state estimation; community phylogeny; dispersal ability; experimental manipulation; habitat preference; phylogenetic signal; phylogeny; springtail; soil preference; succession; transfer experiment 


\section{Introduction}

Composition of biotic community is influenced by both ecological and evolutionary processes (Vellend, 2010, 2016). In a metacommunity, local communities are connected via individual dispersal, interacting with habitat selection processes such as environmental filtering and species interactions that together drive local community composition (Leibold et al. 2004). In local communities, species possess a number of traits reflecting both habitat selection and dispersal processes. Traits associated with habitats, e.g. physiological attributes, may reflect environmental requirements of a species. Other traits such as morphological characters may directly link to species dispersal abilities in a habitat (Ponge et al. 2006, Auclerc et al. 2009, Pey et al. 2014). However, species traits not only are the characters which contemporary assembly processes are based on, but also reflect adaptation of species to the environment during evolutionary history, thus showing phylogenetic signal (Liu et al. 2015). Therefore, contemporary ecological processes, such as habitat selection and dispersal, work on (or relate to) species traits that have been shaped by past processes, and result in current patterns of community assembly.

Relative strengths of assembly processes may vary during colonization of species in new habitats, resulting in dynamic changes in community assembly patterns with time (Emerson and Gillespie 2008, Purschke et al. 2013, Li et al. 2014). At early successional stages, species arrive via dispersal from source habitats; species with high dispersal ability recolonize faster than poor dispersers (Emerson and Gillespie 2008). At these stages dispersal likely overwhelms the influence of local environments, resulting in the "mass effect" scenario, i.e., similar compositions of local communities. In later stages of succession, communities likely follow deterministic trajectories influenced by habitat selection, i.e., environmental filtering and biotic interactions (Purschke et al. 2013, Letten et al. 2014). Selection by the habitats results in the "species sorting" scenario, in which community compositions correlate with local abiotic and/or biotic factors.

To uncover the processes predominant during succession, community phylogeny offers an analytical framework, in which phylogenetic distances of species are used as a surrogate for the differences in traits, assuming that process-related traits exhibit phylogenetic signal (Cavender-Bares et al. 2009, Cadotte et al. 2013, de Bello et al. 2015). If traits are phylogenetically conserved (i.e., related species share similar traits), a community composed of phylogenetically closely related species suggests environmental filtering as major structuring force. In contrast, low relatedness between coexisting species suggests the dominance of biotic interactions, such as competition or niche partitioning (Webb et al. 2002, Cavender-Bares et al. 2009). Furthermore, a phylogeny-weighted community matrix (Pillar and Duarte, 2010) allows to measure phylogenetic dissimilarities between local communities. This matrix, combined with principle coordinate analysis, generates principal coordinates of phylogenetic structure of a metacommunity, allowing to identify phylogenetic clades associated with different habitats (Duarte 2011, Duarte et al. 2016).

Compared to studies on aboveground community succession, assembly processes of belowground community are little known, despite the tremendous biodiversity in soil ecosystems (Anderson 1975, 
Bardgett and van der Putten 2014). Collembola (springtails) are among the most abundant and diverse soil invertebrates which contribute to organic matter decomposition processes and nutrient cycling (Petersen and Luxton 1982, Rusek 1998, Schaefer et al. 2009). Distinct community composition of Collembola in different habitats suggests that assembly processes are related to local abiotic and biotic factors (Caruso et al. 2013, Salmon et al. 2014). Collembola communities also change along successional gradients (Dunger et al. 2004, Huebner et al. 2012, Perez et al. 2013), likely following the "mass effect" scenario via frequent dispersal from the source habitats (Ingimarsdóttir et al. 2012). After arrival in new habitats, however, local environmental conditions likely drive communities in certain directions. As indicated by a field observational study on Collembola assembly processes (Chapter 4) using community phylogenetic approaches, Collembola assemblages are likely driven by environmental filtering in disturbed environments, while niche partitioning is more prominent in a stable habitat. However, to further understand assembly processes of Collembola during succession, a combined approach of community phylogeny and manipulative experiments is needed.

The experiment of Auclerc et al. (2009) offers a model system to examine assembly processes during succession of soil animal community in contrasting habitats. In this study, the authors eradicated animals from meadow and forest soil blocks, and then transferred the soil blocks back to the original habitat or to new habitats (Table 5.1). Individual Collembola species were ascribed to different groups in respect of dispersal ability and habitat preferences based on monitoring species recolonization in the soil blocks. In the present study, we took a community perspective with a focus on assembly processes of Collembola recolonizing the soil blocks. We reanalyzed the data of Auclerc et al. (2009) using (meta)community phylogenetic approaches. We analyzed phylogenetic signal and ancestral states of dispersal abilities and environmental preferences of the species examined in Auclerc et al. (2009), to infer how the ecological preferences of Collembola and their dispersal abilities in habitats have evolved. Then, we inferred assembly processes of Collembola communities inhabiting meadow and forest and at different successional stages. We examined effects of soil origin on community succession patterns and assembly processes in defaunated blocks, as well as the fate of indigenous species after soil blocks were transferred to a new habitat.

We hypothesized that (1) community structures and phylogeny-weighted structures both differ between meadow and forest but not between sampling time; communities in meadow exhibit phylogenetic clustering due to environmental filtering resulting from disturbances, e.g. mowing and grazing, while forest communities show phylogenetic overdispersion resulting from niche partitioning in a stable environment. (2) At early successional stages drift predominates resulting in random patterns of phylogenetic relatedness in defaunated blocks, while selection drives communities to deterministic patterns at later successional stages. (3) Successional patterns in defaunated soil blocks differ between transferred soil blocks and those remained in the original habitat. (4) Community structures in untreated, transferred soil blocks change gradually from those installed in the original habitat to those in the transferred habitat (Table S5.1). 


\section{Materials and Methods}

\section{Collembola communities}

We reanalyzed the data of Collembola community used in Auclerc et al. (2009). The original experiment was conducted between December 2005 and June 2006 in Morvan Regional Natural Park in Burgundy, France. Sixty soil blocks $(15 \mathrm{~cm}$ in diameter and $10 \mathrm{~cm}$ in depth) were sampled from a meadow and another 60 soil blocks from a mixed forest nearby. For each habitat, 30 soil blocks were frozen at $-20^{\circ} \mathrm{C}$ for one week to eradicate soil animals, and the other 30 blocks were left undisturbed. Fifteen defaunated and 15 undisturbed blocks were installed back to their original habitat, while the other 15 defaunated and 15 untreated blocks were transferred to the respective other habitat. The manipulations resulted in eight treatments with 15 soil blocks each (Table 5.1). The soil blocks were sampled again in three time intervals: one week (T1), one month (T2) and six months (T3) after installation. For each sampling interval, five blocks per treatment were randomly selected and used as replicates in the following statistical analyses. Soil animals were extracted from the blocks using heat and Collembola were identified to species level. For more details on the experiment see Auclerc et al. (2009).

\section{Table 5.1}

Eight treatments used in Auclerc et al. (2009). Soil blocks were sampled from meadow or forest, defaunated or untreated and installed to the original or the respective other habitat. For example, WMF indicates untreated soil blocks with fauna (W) originated from meadow $(\mathrm{M})$ and installed in forest $(\mathrm{F})$. Each of the treatments was replicated in 15 soil blocks. Five replicates per treatment were sampled at each of the three intervals, T1 (one week), T2 (one month) and T3 (six months) after soil block installation.

\begin{tabular}{llcc}
\hline \multirow{2}{*}{ Habitat } & Defaunation & \multicolumn{2}{c}{ Soil block installation to } \\
\cline { 3 - 4 } Meadow & Untreated & Original habitat & Respective other habitat \\
\cline { 2 - 4 } Forest & Defaunated & WMM & WMF \\
& Untreated & OMM & OMF \\
& Defaunated & WFF & WFM \\
& & OFF & OFM \\
\hline
\end{tabular}

\section{Collembola phylogeny}

Forty-nine species of Collembola were found in the untreated blocks installed in the original habitats (WFF and WMM; Table 5.1) and were used as the regional species pool in the community phylogenetic analyses. Collembola phylogeny of the regional species pool was constructed using six genetic markers, including 18S rRNA, 28S rRNA (D1, D2 and D3-D5 regions), Histone H3 and Cytochrome Oxidase I (COI). Sequences were downloaded from Genbank with the Accession Number listed in Table S5.2. For species for which none of the above molecular sequences were available, sequences of congeneric species were used. In case of lack of congeneric sequences, taxa were grafted to the most recent common ancestors according to the taxonomy (Table S5.2). 
Sequences of each marker were aligned with outgroup taxa Zygentoma (Insecta), Machilis (Insecta: Archaeognatha) and Callibaetis (Insecta: Palaeoptera) using R functions AlignSeqs and AdjustAlignment for $18 \mathrm{~S}$ and 28S rRNA (package "DECIPHER"; Wright 2015) and msa for Histone H3 and COI, setting gap opening penalty to 15 and gap extension penalty to 6.6 (package "msa"; Bodenhofer et al. 2015). For each genetic marker the aligned sequences were trimmed to the same length. The best model of sequence evolution of each genetic marker was estimated using jModelTest 2.1.4 on the basis of the Akaike information criterion (AIC; Guindon and Gascuel, 2003, Darriba et al. 2012). Ribosomal 18 S and 28S markers and COI were fitted with GTR $+\mathrm{I}+\mathrm{G}$ model, while Histone $\mathrm{H} 3$ was fitted with $\mathrm{SYM}+\mathrm{I}+\mathrm{G}$. In each marker set, terminal gaps at the beginning and the end of sequences varied in length and were replaced by "?", and all the six markers was concatenated in a supermatrix (3,073 bp) using SequenceMatrix 1.8 (Vaidya et al. 2011). Collembola phylogeny was inferred using Bayesian Inference (BI; MrBayes 3.2.4; Ronquist et al. 2012), setting the model of sequence evolution separately for the six markers, two independent runs, four chains, 2,000,000 generations, 0.1 temperature and 0.5 burn-in fraction; other parameters were set as default.

The resulting tree (Figure S5.1a) was transformed to an ultrametric tree using a penalized likelihood approach by assuming different models of substitution rate variation among branches, i.e., correlated, relaxed, discrete and strict clock models, using the function cbronos implemented in the $\mathrm{R}$ package "ape" (Paradis et al. 2004). The best ultrametric tree was selected based on the smallest PHIIC value, a criterion analogous to AIC reflecting the best model fit to the data (Paradis 2013). The ultrametric tree based on the strict clock model was selected, and the species without available sequences (Gisinianus flammeolus and Stenognathellus denisi) were grafted to the most recent common ancestors according to the taxonomy using the function add.species.to.genus implemented in the R package "phytools" (Revell 2012). This tree was then used in comparative analyses of species preferences for habitats and phylogenetic analyses of community (Figure S5.1b).

\section{Phylogenetic comparative methods}

Collembola preferences for habitat and soil and dispersal abilities in meadow and forest reported in Auclerc et al. (2009) were tested for phylogenetic signal (Table S5.2). Species for which data on soil preference were lacking were removed from the corresponding analysis. Characters of species were mapped onto the phylogenetic tree and phylogenetic signal was measured using Pagel's lambda (Pagel 1999, Freckleton et al. 2002) by the function fitDiscrete implemented in R package "geiger" (Harmon et al. 2008). An appropriate model of character evolution was estimated from one of the equal-rates (ER), symmetric (SYM) and all-rates-different (ARD) models using likelihood comparison. The ER model was selected for habitat and soil preferences, while the SYM model was selected for dispersal ability in meadow and forest. A star-like tree (lambda 0) was then transformed from the original tree (lambda 1). Likelihoods of the distribution of character states among species were compared, given the lambda 1 and lambda 0 trees. If the lambda 0 tree was rejected, the character showed phylogenetic signal. An optimal 
lambda value was then estimated which represented the strength of phylogenetic signal (Pagel 1999, Freckleton et al. 2002).

For the characters exhibiting phylogenetic signal, ancestral states of characters were estimated using stochastic character mapping (Huelsenbeck et al. 2003, Bollback 2006) by the function make.simmap implemented in the R package "phytools" (Revell 2012). Transition of character state was assumed following the ER model. Prior distribution on root node was estimated from tip character states. Transition matrix $\mathrm{Q}$ was sampled 1,000 times from the posterior probability distribution using Bayesian MCMC. Then, 1,000 stochastic maps were simulated which were conditioned by the sampled value of $\mathrm{Q}$. Numbers of character state transitions in the tree were reported as mean and median, and posterior probabilities of character states were mapped to the tree nodes.

\section{Community composition and phylogenetic structure}

For each community assembly hypothesis, a respective metacommunity was defined as a combination of several treatments (Table S5.1). Differences in community compositions were calculated based on Bray-Curtis dissimilarity between communities, resulting in a phylogeny-unweighted distance matrix. Effects of treatment and sampling interval on community compositions were tested by distance-based multivariate analysis of variance (ADONIS) with 9,999 permutations using the function adonis implemented in the R package "vegan" (Oksanen et al. 2015). If treatments had significant effects on community compositions, principle coordinate analysis (PCoA) was performed to visualize community compositions using the function pcoa implemented in the R package "ape" (Paradis et al. 2004). Pearson correlation coefficients of site scores and species abundance at each PCoA axis were calculated using function cor.test. $P$-values of correlation tests were adjusted using Benjamini and Hochberg corrections (BH; Benjamini and Hochberg 1995). Treatment effects on site scores of the PCoA axes were tested using Kruskal-Wallis rank sum test followed by the Dunn's test with $\mathrm{BH}$ corrections for multiple comparisons.

Furthermore, community compositions were weighted by species phylogenetic relationships using the phylogenetic fuzzy-weighting method (Pillar and Duarte 2010) implemented in the $\mathrm{R}$ package "SYNCSA"; Debastiani and Pillar 2012). ADONIS was then used to test treatment effects on the phylogeny-weighted community compositions. If treatments had significant effects, phylogeny-weighted community compositions were applied to PCoA using the function $p c p s$ implemented in the R package "PCPS" (Debastiani and Duarte 2014), resulting in principal coordinates of phylogenetic structures (PCPS) of the respective metacommunity. Pearson correlation coefficients of site scores and species abundance at each PCPS axis were tested as described above. Treatment effects on PCPS using KruskalWallis rank sum test followed by the Dunn's test were tested as described above. 


\section{Community phylogenetic relatedness}

Phylogenetic relatedness of coexisting species in local communities was calculated based on species pairwise phylogenetic distances obtained from the ultrametric tree using the function cophenetic.pbylo implemented in R package "ape" (Paradis et al. 2004). Mean pairwise phylogenetic distance (MPD) and mean nearest taxon distance (MNTD) of a local community were calculated and Net Relatedness Index (NRI) and Nearest Taxon Index (NTI) were calculated based on MPD and MNTD to represent overall species relatedness and terminal species relatedness of a local community, respectively (Webb 2000). The NRI is a negative value of the standardized effect size of the MPD, for which the observed MPD was standardized by 999 simulated MPDs generated by drawing species with equal probability from the phylogenetic distance matrix ("phylogeny.pool" null model). Similarly, the NTI is a negative value of the standardized effect size of the MNTD, for which the observed MNTD was standardized by 999 simulated MNTDs. A positive NRI or NTI indicates more closely related species as compared to the "null" communities. A negative NRI or NTI, on the contrary, indicates more distantly related species in a local community. NRI and NTI were calculated using the function ses.mpd and ses.mntd implemented in R package "picante", respectively (Kembel et al. 2010), based on species presence/absence or abundance data.

To infer community assembly processes in each treatment, Student's t-test was used to test mean phylogenetic relatedness of the five replicates of a treatment against " 0 ", which indicates a randomly assembled community. $P$-values were adjusted by the number of treatments within a metacommunity using BH corrections. Kruskal-Wallis rank sum test was applied to test effects of treatments on NRI or NTI, followed by the Dunn's test with BH corrections.

\section{Results}

\section{Evolution of species preferences for habitats and dispersal abilities}

Among the four characters published in Auclerc et al. (2009), only soil preference and dispersal ability in meadow exhibited phylogenetic signal (soil preference: lambda $=0.881, P=0.003$; dispersal ability in meadow: lambda $=0.498, P=0.020$; Figure 5.1a, $\mathbf{b}$ ).

The most likely ancestral state of soil preference of Collembola was for the meadow soil. The median number of soil preference changes was 21 . The median number of transitions from meadow specialist to soil generalist was eight and for the opposite direction it was three, while from meadow specialist to forest specialist it was five and in the opposite direction it was two. There was a single transition between soil generalist and forest specialist for both directions (Table S5.3). The ancestor of Neanuridae and the ancestor of Lepidocyrtidae were likely soil generalists, while the ancestor of Isotomidae was likely a meadow specialist (Figure 5.1a).

Ancestral state of Collembola dispersal ability in meadow was likely either M0 or M1. Transitions of dispersal ability in meadow occurred more often between M0 and M1 and between M0 and M4 than the 

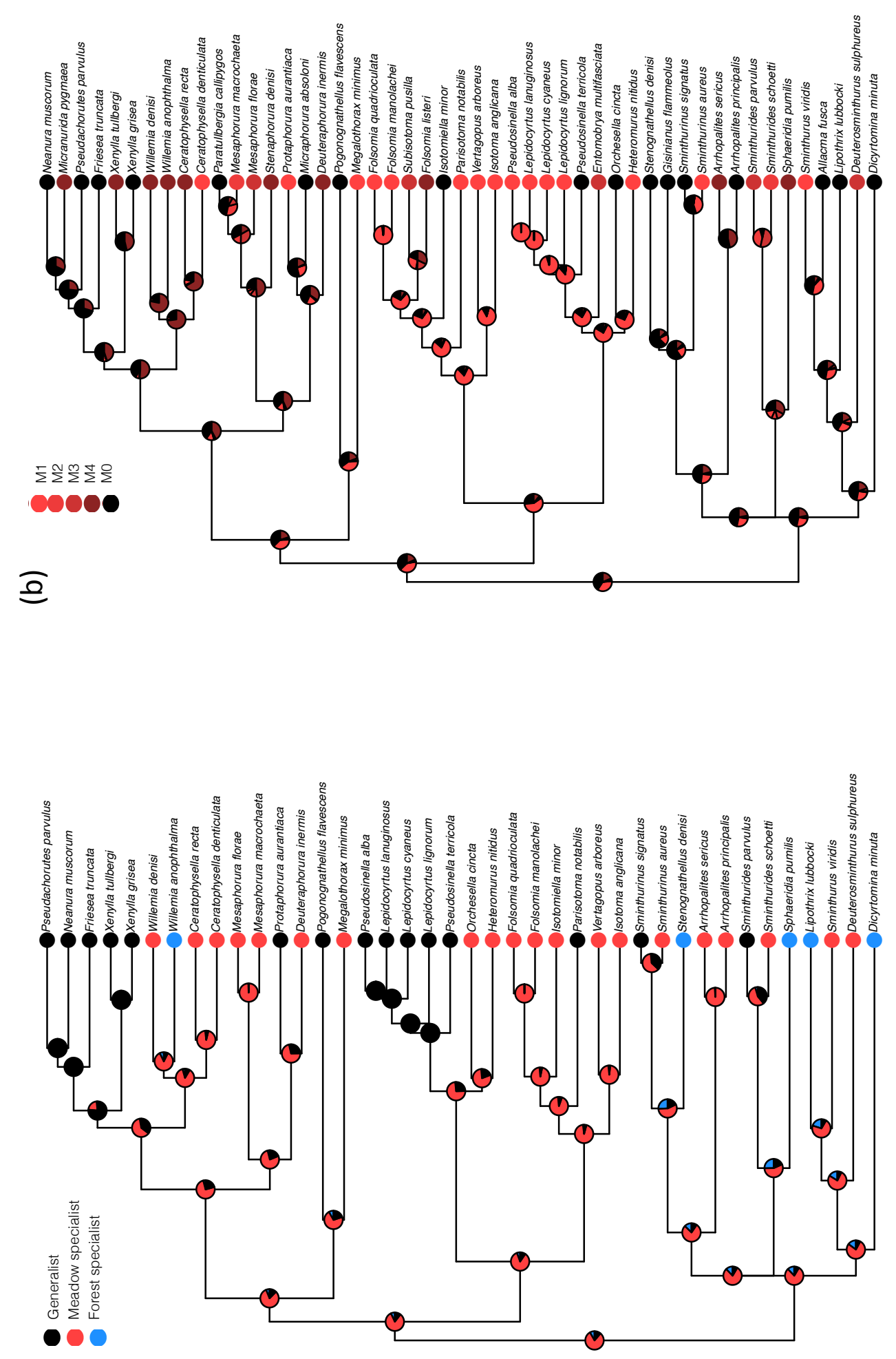

$\widetilde{0}$ 
other state transitions (Table S5.4). The ancestor of Isotomidae and Entomobryoidea was likely a fast disperser in meadow (M1), while the ancestor of Poduromorpha was likely a slow or poor disperser in meadow (M4 and M0). The ancestor of Symphypleona, however, was likely a poor disperser in meadow (M0; Figure 5.1b).

\section{Habitat effects on Collembola communities}

Both community structures and phylogeny-weighted structures differed significantly between meadow (WMM) and forest (WFF) but not between sampling intervals (Table S5.5). Site scores on PCoA 1 and those on PCPS 1, PCPS 3 and PCPS 5 significantly differed between meadow and forest (Figure 5.2a, b, Table S5.6). Phylogenetic gradient along PCPS 1 was mostly correlated with Entomobryoidea and Isotomidae which were associated with forest, and Poduromorpha and Symphypleona which were associated with meadow. PCPS 3 was positively correlated with Poduromorpha and negatively with Symphypleona (Figure 5.2b). Communities in meadow showed phylogenetic overdispersion, while in forest they were phylogenetic clustered as indicated by NRI using species presence/absence data, with a significant difference between meadow and forest. When species abundances were considered, NRI increased in both habitats, indicating that abundant species in both habitats were close relatives. Mean NTI of communities did not differ significantly between meadow and forest when species presence/ absence data were used. However, abundant species of forest communities exhibited phylogenetic clustering, resulting in the NTI being higher in forest than in meadow (Table 5.2).

\section{Successional patterns of community structures}

Community structures differed during succession in both meadow and forest, mainly due to increased abundance of a few species (Figure 5.3a, c, Table S5.5). In contrast, phylogeny-weighted structures in meadow did not differ significantly with successional stages (Figure 5.3b, Table S5.5). However, phylogeny-weighted structures in forest differed between OFF at T1 and WFF (Table S5.6). The OFF communities at T1 were associated with Symphypleona and Entomobryoidea, while WFF communities were associated with Isotomidae and Poduromorpha (Figure 5.3d).

In meadow, NRI using species presence/absence data decreased from T1 to T3 and to the WMM where local communities showed phylogenetic overdispersion. However, when abundances were considered, although NRI did not differ from 0 in all successional stages, NRI at T3 was significantly higher than that at T2 and WMM. In contrast, NTI of OMM at T2 and of WMM show phylogenetic overdispersion but were not different from each other. In forest OFF communities, although phylogenetic relatedness based on species presence/absence data did not differ from that of a random pattern, abundance-weighted NRI and NTI showed significant phylogenetic clustering in each sampling interval, indicating that abundant species were phylogenetically closely related. The abundance-weighted NRI in OFF at T2 was the lowest among all the successional stages, while the abundance-weighted NTI 
in OFF at T1 was the highest. Interestingly, NTI using species presence/absence data decreased from T1 to T3 and to WFF the lowest (Table 5.2).
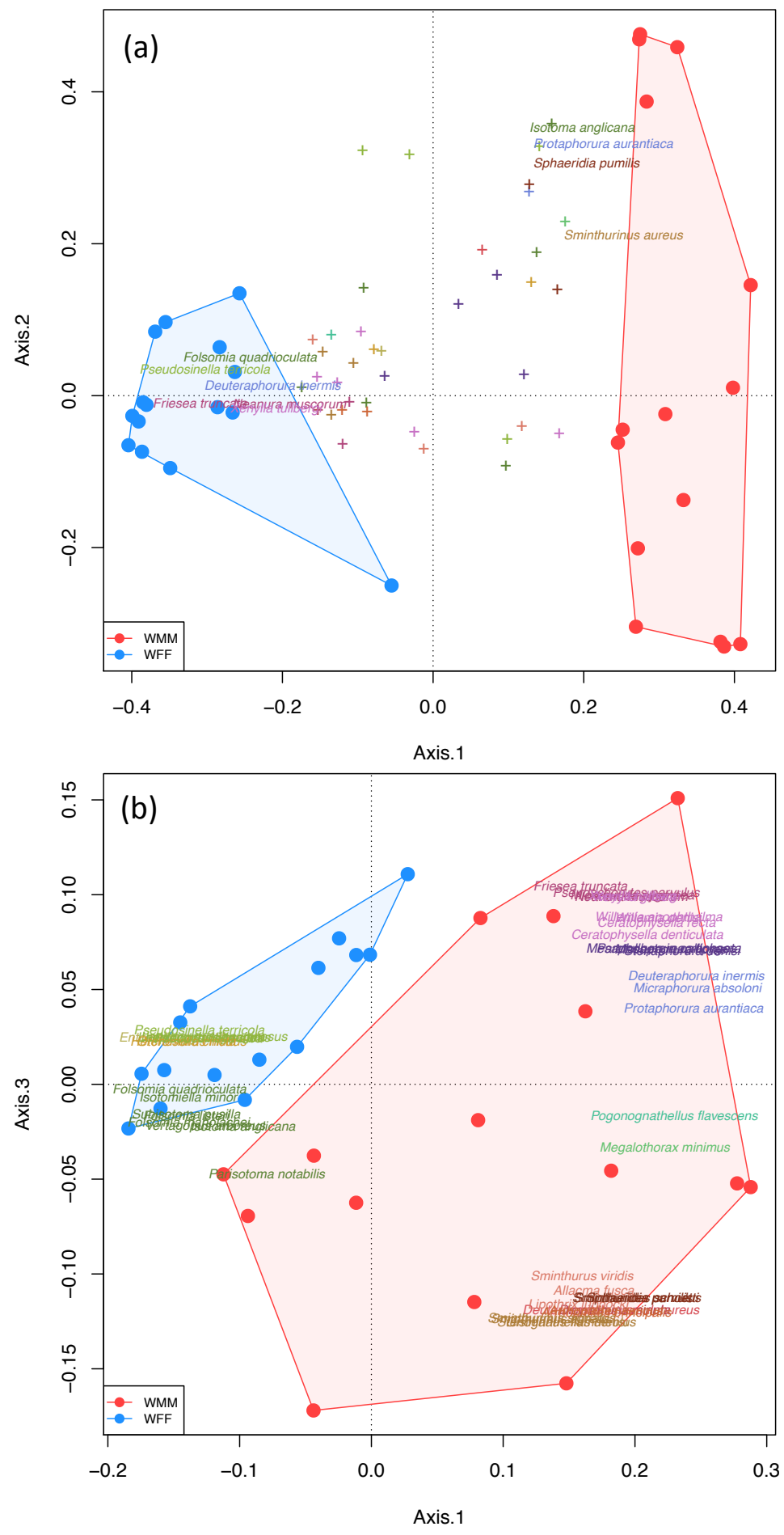

Figure 5.2

Scatter diagram of principle coordinates of (a) community structure (PCoA) and (b) phylogenetic-weighted structure (PCPS) of Collembola communities occurring in the untreated soil blocks installed in meadow (WMM) and forest (WFF) pooled for sampling times. Polygons encompass replicates of the same treatment. Only Collembola species significantly correlated with the axes are plotted with names and the others with cross symbols. 


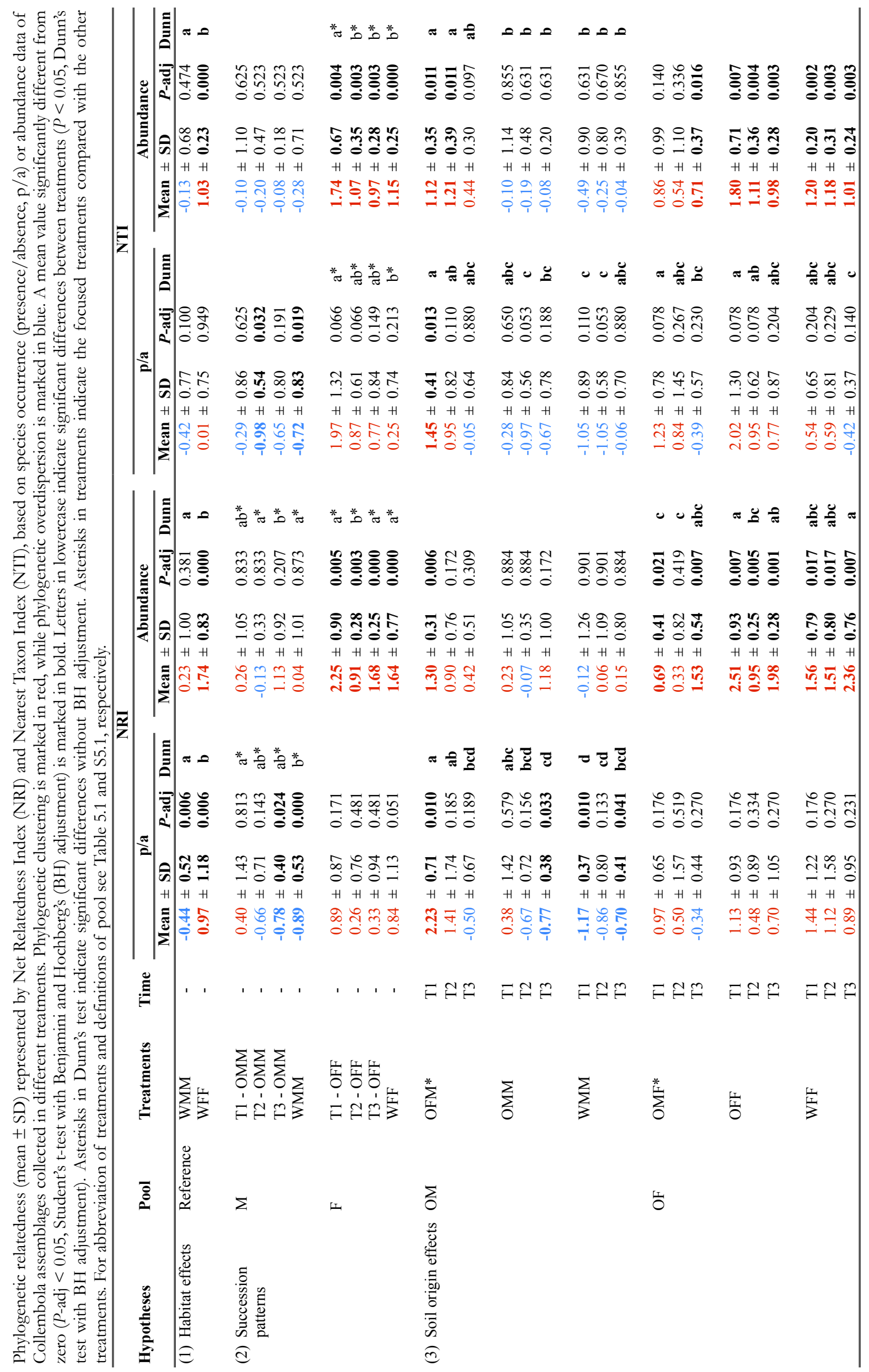




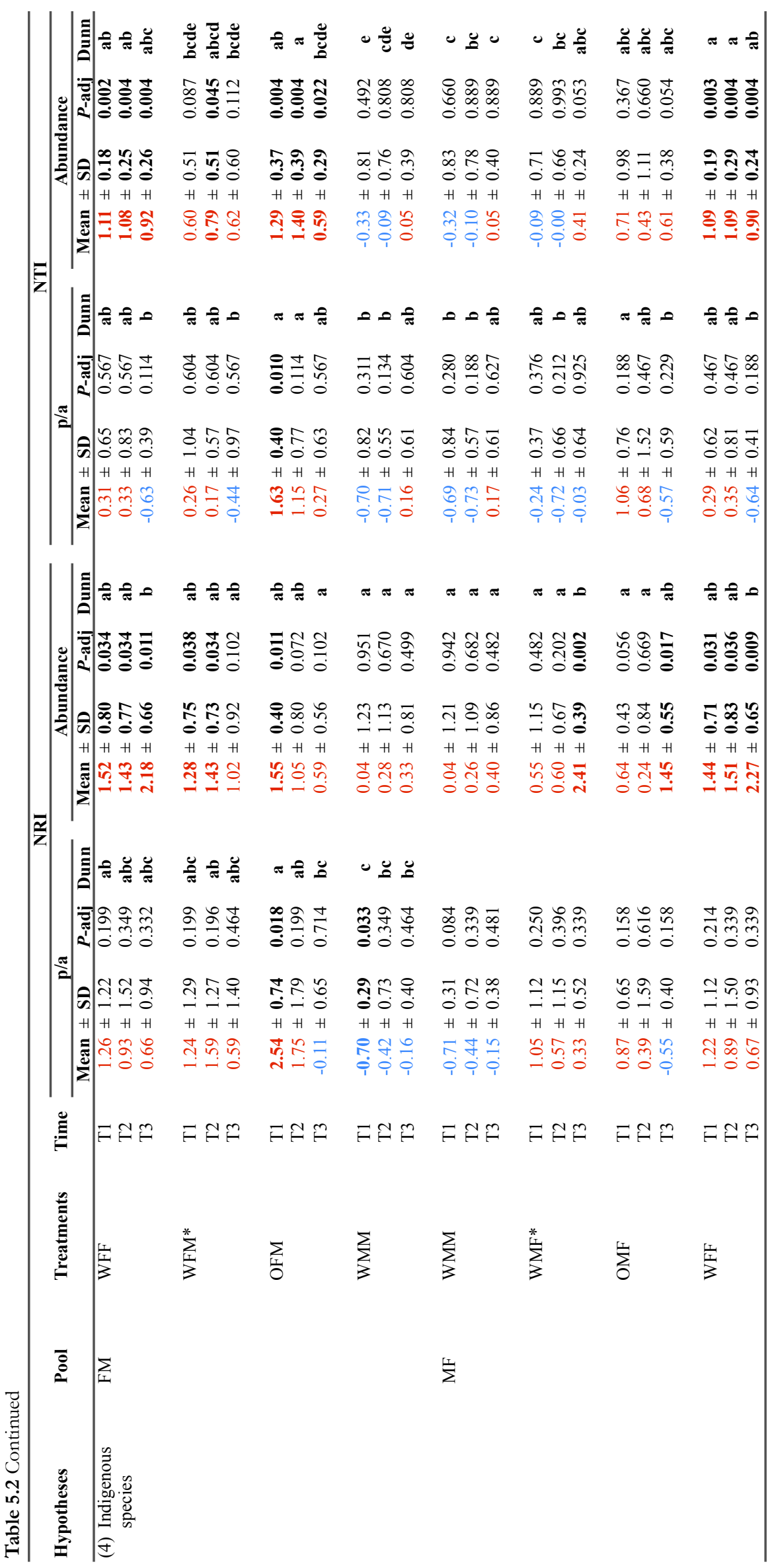



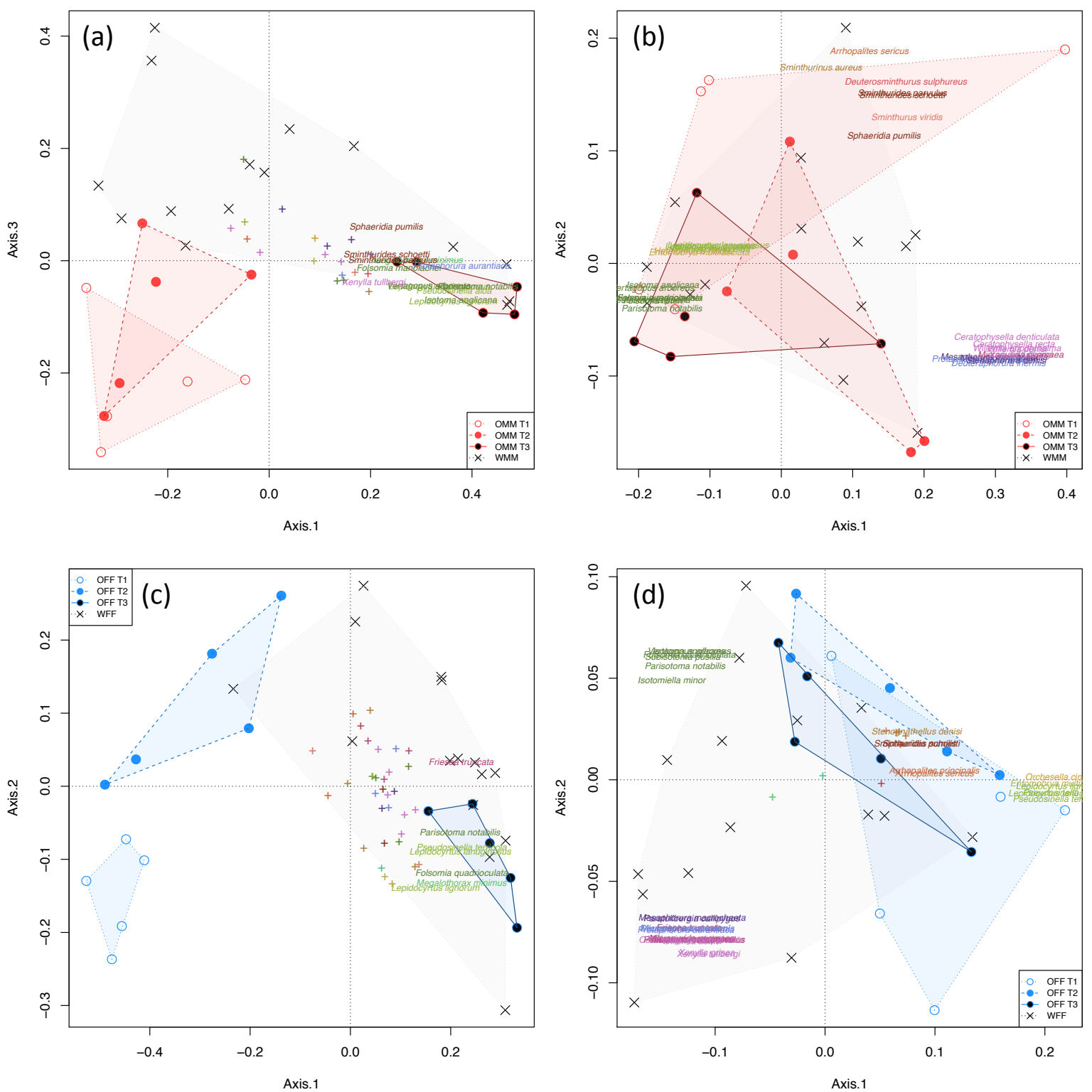

Figure 5.3

Scatter diagram of principle coordinates of (a) community structure (PCoA) and (b) phylogenetic-weighted structure (PCPS) of Collembola communities occurring in defaunated meadow soil blocks (OMM) sampled one week (T1), one month (T2) and six months (T3) after installation and in untreated meadow soil blocks (WMM). Scatter diagram of principle coordinates of (c) community structure (PCoA) and (d) phylogenetic-weighted structure (PCPS) of Collembola communities occurring in defaunated forest soil blocks (OFF) sampled one week (T1), one month (T2) and six months (T3) after installation and in untreated forest soil blocks (WFF). Polygons encompass replicates of the same sampling time. Only Collembola species significantly correlated to the axes are plotted with names and the others with cross symbols.

\section{Effects of soil origin on community compositions}

Communities of OFM, OMM and WMM, all surrounded by meadow soil, differed in their assembly patterns but this varied with sampling intervals (Table S5.5). Site scores of OFM communities at T1 differed from those at T3 on PCoA 1. OFM communities differed from OMM communities at T2 for 
site scores on PCoA 4 which were positively correlated with the abundance of Ceratophysella denticulata (Figure 5.4a, Table S5.6). In contrast, phylogeny-weighted structures were influenced by treatments but not by sampling intervals (Table S5.5, S5.6). Soil originating from forest was associated with Entomobryoidea that immigrated from the meadow surrounding (Figure 5.4b). OFM communities exhibited phylogenetic clustering that decreased with successional stages, as indicated by NRI using presence/absence data. However, NRI of OFM communities did not differ significantly from those of OMM in all three sampling intervals. Furthermore, NTI of OFM at T2 was significantly higher than that
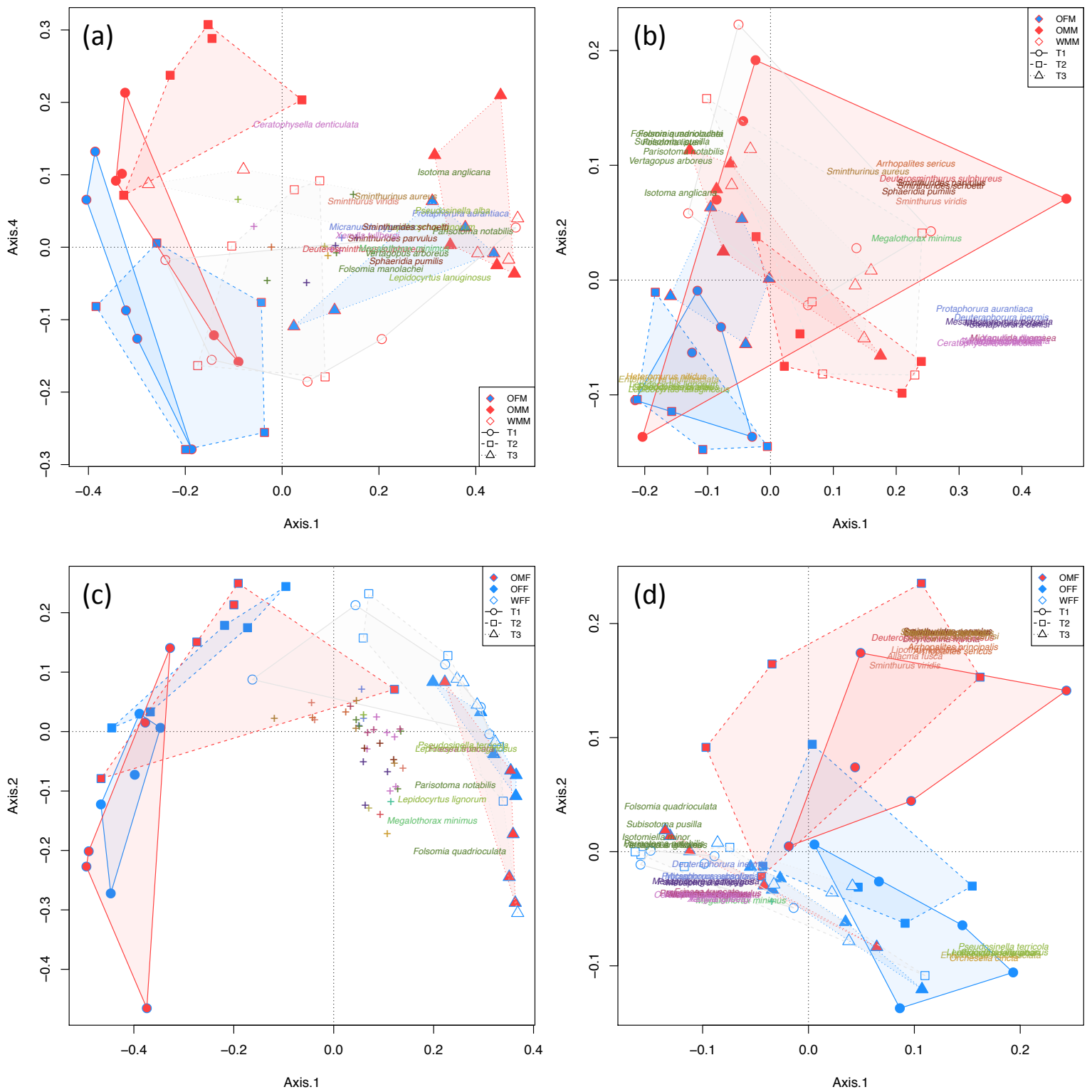

Figure 5.4

Scatter diagram of principle coordinates of (a) community structure (PCoA) and (b) phylogenetic-weighted structure (PCPS) of Collembola communities surrounded by meadow, and of (c) community structure (PCoA) and (d) phylogenetic-weighted structure (PCPS) of Collembola communities surrounded by forest. Polygons encompass replicates of the same treatment per sampling time. Only Collembola species significantly correlated to the axes are plotted with names and the others with cross symbols. 
of OMM using species presence/absence data, while abundance-weighted NTI at both T1 and T2 were higher than that of OMM at the corresponding sampling intervals. This indicates that while OFM communities were phylogenetically clustered at T1, phylogenetic relatedness decreased with succession to a more random pattern at T3; compared with OMM, OFM exhibited more phylogenetic clustering (Table 5.2).

OMF, OFF and WFF communities, all surrounded by forest soil, differed in their assembly patterns but this again varied with sampling intervals (Table S5.5). OMF communities did not differ from OFF communities in any sampling interval (Table S5.6), although OMF communities changed with successional stages (Figure 5.4c). This indicates that meadow soil in the transferred blocks to forest habitat did not influence community structures significantly. Similarly, phylogeny-weighted structures differed between treatments and between successional stages, with these two factors depending on each other (Table S5.5). Differences between OMF and OFF communities were significant at T1 on PCP2 (Table S5.6). On that axis OMF communities were associated with Symphypleona while those of OFF with Entomobryoidea (Figure 5.4d). At T1 mean abundance-weighted NRI of OMF communities was significantly lower than that of OFF communities but both were higher than 0 . The species presence/ absence NTI of OMF communities decreased significantly with successional stage but did not differ from random. Abundant species in the OMF communities exhibited phylogenetic clustering at T3 as indicated by both NRI and NTI (Table 5.2).

\section{Indigenous species}

Indigenous species originating from one habitat but transferred to another habitat contributed to differences in community composition as compared with defaunated soil blocks, but this varied with time (Table S5.5). Collembola communities in forest blocks transferred to meadow (WFM) resembled those of their original forest habitat (WFF), but differed from those of the meadow (WMM) at each sampling interval, as indicated by site scores on PCoA 1 (Figure 5.5a, Table S5.6). Phylogeny-weighted structures differed between treatments but not between sampling intervals (Table S5.5). Furthermore, phylogenyweighted structures of WFM communities at T3 resembled that of WMM communities but differed from that of WFF communities on PCPS 1, which was positively correlated with Poduromorpha, Tomoceridae and Neelidae, and negatively with Isotomidae and Entomobryoidea (Figure 5.5b). Phylogenetic relatedness in WFM communities did not differ from those of the other treatments at all sampling intervals (Table 5.2).

Collembola communities in meadow blocks transferred to forest (WMF) resembled those of their original meadow habitat (WMM) at T1 and T2, but were more similar to WFF communities at T3. Significant differences between WMF and OMF communities occurred at T2, when WMF communities resembled WMM communities and OMF communities resembled WFF communities. At T3 both WMF and OMF communities resembled WFF communities (Figure 5.5c, Table S5.5, S5.6). Phylogeny- 
weighted structures of WMF could not be differentiated from either WMM or WFF at each sampling interval. However, WMF communities differed from OMF communities at T1, as indicated by PCPS 1 and PCPS 2 (Table S5.6). WMF communities at T1 were associated with the clade composed of Poduromorpha plus Tomoceridae and Neelidae, while OMF communities at T1 were associated with the other basal phylogenetic clades, e.g. Entomobryoidea and Isotomidae (Figure 5.5d). Mean abundanceweighted phylogenetic relatedness of WMF communities at T3 was significantly higher than 0 as well as that of WMM communities, but not different from that of WFF and OMF communities as indicated by abundance-weighted NRI (Table 5.2).
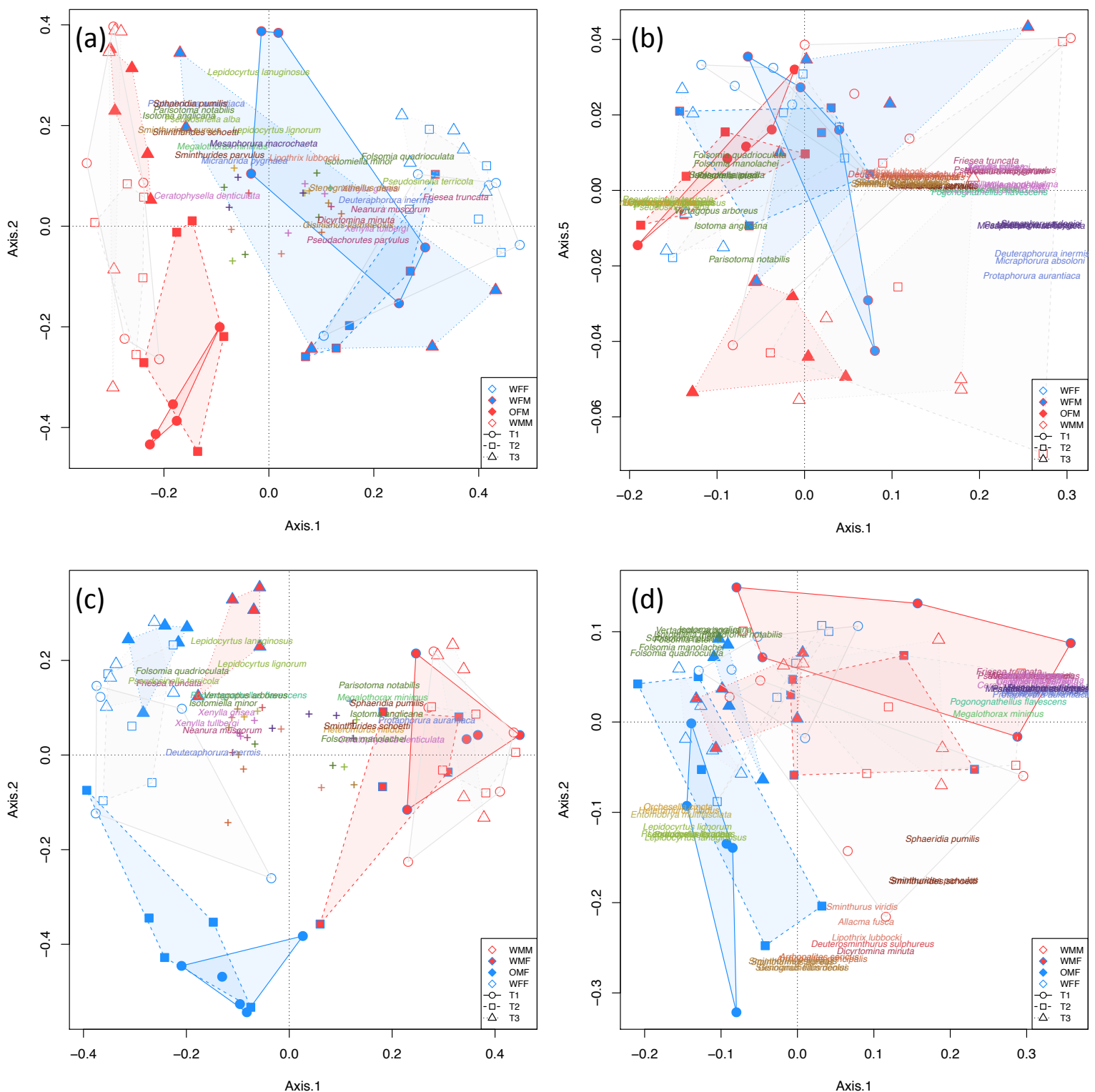

Figure 5.5

Scatter diagram of principle coordinates of community structure (a) and (c), and phylogenetic-weighted structure (b) and (d) of Collembola communities. Polygons encompass replicates of the same treatment per sampling time. Only Collembola species significantly correlated to the axes are plotted with names and the others with cross symbols. 


\section{Discussion}

\section{Evolution of Collembola dispersal abilities and environmental preferences}

Phylogenetic signal found in Collembola dispersal ability in meadow suggests that related species displayed similar ability to move from the surrounding to the defaunated soil blocks in meadow habitat. Generally, most of Isotomidae and Entomobryoidea recolonized the defaunated soil blocks, while most species from Neanuridae and Hypogastruridae were poor dispersers. Dispersal ability in meadow likely reflected morphological characters of different taxa. Isotomidae and Entomobryoidea usually are elongate and possess long furca, while Neanuridae and Hypogastruridae are stout with short furca. Since these morphological characters were fixed within taxonomic groups (Chapter 2), ancestor of Isotomidae and Entomobryoidea in a meadow-like habitat was likely a fast disperser, while that of Poduromorpha was likely a poor disperser. Similarly, preference of Collembola for soil types exhibited phylogenetic signal. Ancestral character estimation showed that ancestor of Collembola was likely a meadow soil specialist but this changed to soil generalists several times during species diversification, while changes from generalist to meadow soil specialist occurred less frequently. Notably, changes from meadow soil specialists to forest soil specialists occurred frequently, while changes from soil generalists to forest soil specialists and vice versa rarely occurred. Compared to other soil animal taxa such as oribatid mites (Oribatida), Collembola recover faster after drought (Lindberg and Bengtsson 2005) and more tolerate fluctuations in temperature and soil moisture (Tsiafouli et al. 2005). Accordingly, Collembola might have adapted to meadow-like habitats characterized by fluctuations in abiotic environmental factors.

In the paper of Auclerc et al. (2009) Collembola dispersal ability in meadow and forest was defined by the time at which the species reappeared in the defaunated blocks (OFF, OMM), while soil preference was estimated by difference in abundance of species between forest and meadow soil cores installed in the same habitat. Species abilities to immigrate from the surrounding habitat and to survive in a new habitat are determined not only by their morphological characters, such as legs, antenna, furca and visual apparatus (Ponge et al. 2006), but also by the physiological attributes e.g. those related to moisture tolerance (Kuznetsova 2003). Soil preference identified in the study may be correlated with species dispersal from the surrounding. Therefore, dispersal ability and soil preference in the present study should be considered as a summarized response that is influenced by various functional traits of the species. Determination of these functional traits needs further measurements at individual or population level using both laboratory experiments and field observations (Pey et al. 2014, Moretti et al. 2017). Overall, our study indicates that closely related species shared similar soil preference and dispersal ability in meadow, and phylogenetic signal in these characters likely resulted from niche conservatism in morphological and/or physiological functional traits. 


\section{Hypothesis 1 - Habitat}

Contrary to our Hypothesis 1, communities in meadow showed phylogenetic overdispersion, while those in forest exhibited phylogenetic clustering. These findings also contrast results presented in the previous study (Chapter 4) where Collembola from forests exhibited phylogenetic overdispersion, while those from grasslands showed random patterns. Presumably, the difference between the two studies was due to the different sampling designs. In the previous study, Collembola communities were sampled from replicated forest sites and grasslands, while in the present study communities of each type of habitats were replicated by soil blocks from a single site. This suggests that assembly processes of Collembola differed not only between habitats but may also be site-specific. In the present study, Collembola in forest comprised species of Isotomidae and Entomobryoidea, resulting in coexistence of phylogenetically closely related species, while meadow communities comprised Poduromorpha, Symphypleona, Neelipleona and Tomoceridae - species distantly related to each other. If traits responsible for these processes exhibited phylogenetic signal, Collembola communities of the studied forest in Morvan Regional Natural Park were likely structured by environmental filtering. Future studies need to identify the traits responsible for the observed patterns.

\section{Hypothesis 2 - Succession}

As tested in Hypothesis 1, community structures and phylogenetic relatedness of WMM and WFF did not significantly differed between the three sampling intervals, T1, T2 and T3. Temporal variations in the other treatments, therefore, reflected successional changes of Collembola communities. Consistent with our expectation, community phylogenetic relatedness in meadow as indicated by NRI decreased along successional stages when presence/absence data were used, although phylogeny-weighted structures did not differ significantly. At later stages, communities comprised the species assembled from various clades, though with low abundance. Presumably, Collembola communities in meadow needed a longer period of time, i.e., 6 months (T3), to fill the empty niches of defaunated soil blocks with distantly related species immigrated from the surrounding soil. Niche partitioning, instead of environmental filtering, was likely the predominant process at later successional stages of Collembola community in the meadow habitat.

In contrast, phylogeny-weighted community structures in forest differed between OFF at T1 and WFF, suggesting that habitat sorting was the main driver of Collembola community assembly early during succession (OFF, T1). Environmental filtering, interacting with frequent dispersal of a few species of Entomobryoidea throughout the study period, likely resulted in phylogenetic clustering in Collembola communities in forest. Interestingly, a decrease in abundance-weighted NTI at later successional stages suggests that niche partitioning gradually became stronger. 


\section{Hypothesis 3 - Soil origin}

Community assembly patterns of defaunated soil blocks transferred to the respective other habitat differed from those in the original habitat, suggesting that soil properties influenced community assembly of Collembola. This is in line with the species sorting scenario of metacommunity theory (Leibold et al. 2004). However, the patterns varied with successional stages and also with the habitats where the soil blocks were inserted into. Soil originating from forest transferred to meadow was associated with Entomobryoidea. Presumably, forest soil attracted species of Entomobryoidea with some traits facilitating immigration from the surrounding meadow, resulting in different structures between OFM and OMM. Future studies need to investigate the traits corresponding to the species sorting by forest soil. Furthermore, decrease in phylogenetic relatedness in OFM suggests that environmental filtering ("species sorting") by forest soil in the meadow dominated at the beginning of succession but was less pronounced later on. Continuous immigration of species from the surrounding meadow habitat likely resulted in the "mass effect", and as a consequence, phylogenetic relatedness of OFM communities was similar to that of OMM communities at later successional stages.

The influence of species sorting in meadow soil blocks inserted into forest was weaker than that in forest soil blocks inserted into meadow, as indicated by the similarity between OMF and OFF communities at all three sampling intervals. This is further supported by the only significant difference in PCPS between OMF and OFF at T1, with no differences at later successional stages. At the beginning of succession, meadow soil blocks (OMF) were colonized predominantly by Symphypleona, while forest soil blocks (OFF) were colonized predominantly by Entomobryoidea. The lower abundance-weighted NRI of $\mathrm{OMF}$ at $\mathrm{T} 1$ than that of $\mathrm{OFF}$ indicates that meadow soil might hamper immigration of phylogenetically related species from forest habitat early during succession. Later, frequent immigration of species from the surrounding forest habitat likely resulted in the patterns in which OMF communities resembled OFF communities, and lower NTI (presence/absence data) in OMF communities at later successional stages. Overall, the results suggest that species sorting caused by transferring local soil likely influenced community assembly early in succession, while at later successional stages, mass effect predominantly structured Collembola communities in defaunated and transferred soil blocks.

\section{Hypothesis 4 - Indigenous species}

Community composition of Collembola in untreated forest soil blocks inserted into meadow soil (WFM) resembled those in the original forest habitat (WFF) but differed from those in the meadow (WMM). Although meadow habitat was characterized by more fluctuation in soil temperature and moistures, indigenous Collembola species of forest soil survived the translocation to meadow and might hamper colonization by meadow Collembola species. However, immigration of species from the surrounding meadow soil at T3 (e.g., Poduromorpha, Symphypleona, Tomoceridae and Neelipleona) likely resulted in resemblance between WFM and WMM communities at PCPS 1. In contrast, Collembola 
communities in meadow soil blocks inserted into forest soil (WMF) at T3 changed from meadow communities (WMM) to those of forest soil (WFF), indicating that indigenous meadow species could not survive, except for Isotomidae that not only remained in meadow blocks but also immigrated from the surrounding forest soil. Presumably, the change from a fluctuating to a more stable environment resulted in environmental filtering predominantly structuring Collembola communities.

\section{Outlook}

For the first time we applied community phylogenetic approaches to a field manipulative experiment on Collembola communities where the original animals were removed and recolonization of species was monitored in both native and translocated habitats characterized by different environmental variability. Assembly processes of Collembola along successional trajectories were inferred using phylogenetic relatedness as a surrogate for trait similarity between coexisting species. Since traits are objects that different processes relate to or work on, the next step is to explicitly measure functional traits including morphological, physiological as well as trophic attributes, and to test phylogenetic niche conservatism of the traits. Integrating phylogenetic comparative methods and community phylogenetic and trait-based approaches in both manipulative experiments and field observations allows a deeper understanding of the mechanisms driving and maintaining species coexistence in soil.

\section{Author Contributions}

TWC and SS conceived the ideas; AA, JFP, SB and FD collected the data; TWC and GM analyzed the data; TWC, GM and SS wrote the manuscript.

\section{References}

Anderson, J.M. (1975) The enigma of soil animal species diversity. Progress in Soil Zoology: Proceedings of the 5th International Colloquium on Soil Zoology (ed J. Vaněk), pp. 51-58. Springer Netherlands, Dordrecht.

Auclerc, A., Ponge, J.-F., Barot, S. \& Dubs, F. (2009) Experimental assessment of habitat preference and dispersal ability of soil springtails. Soil Biology and Biochemistry, 41, 1596-1604.

Bardgett, R.D. \& van der Putten, W.H. (2014) Belowground biodiversity and ecosystem functioning. Nature, 515, 505-511.

de Bello, F., Berg, M.P., Dias, A.T.C., Diniz-Filho, J.A.F., Götzenberger, L., Hortal, J., Ladle, R.J. \& Lepš, J. (2015) On the need for phylogenetic "corrections" in functional trait-based approaches. Folia Geobotanica, 50, 349-357.

Benjamini, Y. \& Hochberg, Y. (1995) Controlling the false discovery rate: a practical and powerful approach to multiple testing. Journal of the Royal Statistical Society Series B, 57, 289-300.

Bodenhofer, U., Bonatesta, E., Horejs-Kainrath, C. \& Hochreiter, S. (2015) msa: An R package for multiple sequence alignment. Bioinformatics, 31, 3997-3999.

Bollback, J.P. (2006) SIMMAP: stochastic character mapping of discrete traits on phylogenies. BMC bioinformatics, 7, 88. 
Cadotte, M., Albert, C.H. \& Walker, S.C. (2013) The ecology of differences: assessing community assembly with trait and evolutionary distances. Ecology Letters, 16, 1234-1244.

Caruso, T., Trokhymets, V., Bargagli, R. \& Convey, P. (2013) Biotic interactions as a structuring force in soil communities: evidence from the micro-arthropods of an Antarctic moss model system. Oecologia, 172, 495-503.

Cavender-Bares, J., Kozak, K.H., Fine, P. V \& Kembel, S.W. (2009) The merging of community ecology and phylogenetic biology. Ecology Letters, 12, 693-715.

Darriba, D., Taboada, G.L., Doallo, R. \& Posada, D. (2012) jModelTest 2: more models, new heuristics and parallel computing. Nature Methods, 9, 772.

Debastiani, V.J. \& Duarte, L.D.S. (2014) PCPS - an R-package for exploring phylogenetic eigenvectors across metacommunities. Frontiers of Biogeography, 6, 144-148.

Debastiani, V.J. \& Pillar, V.D. (2012) SYNCSA--R tool for analysis of metacommunities based on functional traits and phylogeny of the community components. Bioinformatics, 28, 2067-8.

Duarte, L.D.S. (2011) Phylogenetic habitat filtering influences forest nucleation in grasslands. Oikos, 120, 208-215.

Duarte, L.D.S., Debastiani, V.J., Freitas, A.V.L. \& Pillar, V.D. (2016) Dissecting phylogenetic fuzzy weighting: theory and application in metacommunity phylogenetics. Methods in Ecology and Evolution, 7, 937-946.

Dunger, W., Schulz, H.-J., Zimdars, B. \& Hohberg, K. (2004) Changes in collembolan species composition in Eastern German mine sites over fifty years of primary succession. Pedobiologia, 48, 503-517.

Emerson, B.C. \& Gillespie, R.G. (2008) Phylogenetic analysis of community assembly and structure over space and time. Trends in Ecology \& Evolution, 23, 619-630.

Freckleton, R.P., Harvey, P.H. \& Pagel, M. (2002) Phylogenetic analysis and comparative data: a test and review of evidence. The American Naturalist, 160, 712-726.

Guindon, S. \& Gascuel, O. (2003) A simple, fast and accurate method to estimate large phylogenies by maximumlikelihood. Systematic Biology, 52, 696-704.

Harmon, L.J., Weir, J.T., Brock, C.D., Glor, R.E. \& Challenger, W. (2008) GEIGER: Investigating evolutionary radiations. Bioinformatics, 24, 129-131.

Huebner, K., Lindo, Z. \& Lechowicz, M.J. (2012) Post-fire succession of collembolan communities in a northern hardwood forest. European Journal of Soil Biology, 48, 59-65.

Huelsenbeck, J.P., Nielsen, R., Bollback, J.P. \& Schultz, T. (2003) Stochastic Mapping of Morphological Characters. Systematic Biology, 52, 131-158.

Ingimarsdóttir, M., Caruso, T., Ripa, J., Magnusdottir, O.B., Migliorini, M. \& Hedlund, K. (2012) Primary assembly of soil communities: disentangling the effect of dispersal and local environment. Oecologia, 170, 745-754.

Kembel, S.W., Cowan, P.D., Helmus, M.R., Cornwell, W.K., Morlon, H., Ackerly, D.D., Blomberg, S.P. \& Webb, C.O. (2010) Picante: R tools for integrating phylogenies and ecology. Bioinformatics, 26, 1463-4.

Kuznetsova, N. (2003) Humidity and distribution of springtails. Entomological Review, 83, 230-238.

Leibold, M.A., Holyoak, M., Mouquet, N., Amarasekare, P., Chase, J.M., Hoopes, M.F., Holt, R.D., Shurin, J.B., Law, R., Tilman, D., Loreau, M. \& Gonzalez, A. (2004) The metacommunity concept: a framework for multi-scale community ecology. Ecology Letters, 7, 601-613.

Letten, A.D., Keith, D.A. \& Tozer, M.G. (2014) Phylogenetic and functional dissimilarity does not increase during temporal heathland succession. Proceedings of the Royal Society B, 281, 2014102.

Li, J., Li, S., Chen, Y., Jia, P., Hua, Z., Wang, S., Song, Y., Liao, B. \& Shu, W. (2014) Phylogenetic structures of soil nematode communities along a successional gradient in an unreclaimed copper mine tailings site. Soil Biology and Biochemistry, 77, 179-186. 
Lindberg, N. \& Bengtsson, J. (2005) Population responses of oribatid mites and collembolans after drought. Applied Soil Ecology, 28, 163-174.

Liu, H., Xu, Q., He, P., Santiago, L.S., Yang, K. \& Ye, Q. (2015) Strong phylogenetic signals and phylogenetic niche conservatism in ecophysiological traits across divergent lineages of Magnoliaceae. Scientific Reports, 5, 12246.

Moretti, M., Dias, A.T.C., de Bello, F., Altermatt, F., Chown, S.L., Azcarate, F.M., Bell, J.R., Fournier, B., Hedde, M., Hortal, J., Ibanez, S., Öckinger, E., Sousa, J.P., Ellers, J. \& Berg, M.P. (2017) Handbook of protocols for standardized measurement of terrestrial invertebrate functional traits. Functional Ecology, 31, 558-567.

Oksanen, J., Blanchet, F.G., Kindt, R., Legendre, P., Minchin, P.R., O’Hara, R.B., Simpson, G.L., Solymos, P., Stevens, M.H.H. \& Wagner, H. (2015) vegan: community ecology package.

Pagel, M. (1999) Inferring the historical patterns of biological evolution. Nature, 401, 877-884.

Paradis, E. (2013) Molecular dating of phylogenies by likelihood methods: A comparison of models and a new information criterion. Molecular Phylogenetics and Evolution, 67, 436-444.

Paradis, E., Claude, J. \& Strimmer, K. (2004) APE: Analyses of phylogenetics and evolution in R language. Bioinformatics, 20, 289-290.

Perez, G., Decaëns, T., Dujardin, G., Akpa-Vinceslas, M., Langlois, E. \& Chauvat, M. (2013) Response of collembolan assemblages to plant species successional gradient. Pedobiologia, 56, 169-177.

Petersen, H. \& Luxton, M. (1982) A comparative analysis of soil fauna populations and their role in decomposition processes. Oikos, 39, 288-388.

Pey, B., Nahmani, J., Auclerc, A., Capowiez, Y., Cluzeau, D., Cortet, J., Decaëns, T., Deharveng, L., Dubs, F., Joimel, S., Briard, C., Grumiaux, F., Laporte, M.-A.A., Pasquet, A., Pelosi, C., Pernin, C., Ponge, J.-F., Salmon, S., Santorufo, L. \& Hedde, M. (2014) Current use of and future needs for soil invertebrate functional traits in community ecology. Basic and Applied Ecology, 15, 194-206.

Pillar, V.D. \& Duarte, L.D.S. (2010) A framework for metacommunity analysis of phylogenetic structure. Ecology Letters, 13, 587-596.

Ponge, J.-F., Dubs, F., Gillet, S., Sousa, J. \& Lavelle, P. (2006) Decreased biodiversity in soil springtail communities: the importance of dispersal and landuse history in heterogeneous landscapes. Soil Biology and Biochemistry, 38, 1158-1161.

Purschke, O., Schmid, B.C., Sykes, M.T., Poschlod, P., Michalski, S.G., Durka, W., Kühn, I., Winter, M. \& Prentice, H.C. (2013) Contrasting changes in taxonomic, phylogenetic and functional diversity during a long-term succession: Insights into assembly processes. Journal of Ecology, 101, 857-866.

Revell, L.J. (2012) phytools: An R package for phylogenetic comparative biology (and other things). Methods in Ecology and Evolution, 3, 217-223.

Ronquist, F., Teslenko, M., van der Mark, P., Ayres, D.L., Darling, A., Höhna, S., Larget, B., Liu, L., Suchard, M.A. \& Huelsenbeck, J.P. (2012) Mrbayes 3.2: Efficient Bayesian phylogenetic inference and model choice across a large model space. Systematic Biology, 61, 539-542.

Rusek, J. (1998) Biodiversity of Collembola and their functional role in the ecosystem. Biodiversity and Conservation, 7 , 1207-1219.

Salmon, S., Ponge, J.-F., Gachet, S., Deharveng, L., Lefebvre, N. \& Delabrosse, F. (2014) Linking species, traits and habitat characteristics of Collembola at European scale. Soil Biology and Biochemistry, 75, 73-85.

Schaefer, M., Migge-Kleian, S. \& Scheu, S. (2009) The role of soil fauna for decomposition of plant residues. Functioning and Management of European Beech Ecosystems (eds R. Brumme \& P.K. Khanna), pp. 207-230. Springer Berlin Heidelberg, Berlin, Heidelberg.

Tsiafouli, M.A., Kallimanis, A.S., Katana, E., Stamou, G.P. \& Sgardelis, S.P. (2005) Responses of soil microarthropods to experimental short-term manipulations of soil moisture. Applied Soil Ecology, 29, 17-26. 
Vaidya, G., Lohman, D.J. \& Meier, R. (2011) SequenceMatrix: Concatenation software for the fast assembly of multi-gene datasets with character set and codon information. Cladistics, 27, 171-180.

Vellend, M. (2010) Conceptual synthesis in community ecology. The Quarterly Review of Biology, 85, 183-206.

Vellend, M. (2016) The Theory of Ecological Communities. Princeton University Press, Princeton.

Webb, C.O. (2000) Exploring the Phylogenetic Structure of Ecological Communities: An Example for Rain Forest Trees. The American Naturalist, 156, 145-155.

Webb, C.O., Ackerly, D.D., McPeek, M.A. \& Donoghue, M.J. (2002) Phylogenies and community ecology. Annual Review of Ecology and Systematics, 33, 475-505.

Wright, E.S. (2015) DECIPHER: harnessing local sequence context to improve protein multiple sequence alignment. BMC Bioinformatics, 16, 322. 


\section{Supplementary Materials}

\section{Table S5.1}

Hypotheses tested in this study and definition of metacommunity (species pool) for each hypothesis. Treatments in comparison are marked in bold. Asterisks in treatments indicate the focused treatments. For abbreviation of treatments see Table 5.1.

\begin{tabular}{|c|c|c|c|}
\hline \multirow{2}{*}{\multicolumn{2}{|c|}{ Hypotheses }} & \multicolumn{2}{|c|}{$\begin{array}{r}\text { Metacommunity (species pool) } \\
\end{array}$} \\
\hline & & \multirow{2}{*}{$\begin{array}{l}\text { Definition (number of species) } \\
\text { Reference: Untreated soil blocks installed in } \\
\text { original habitats (49) }\end{array}$} & \multirow{2}{*}{$\begin{array}{l}\text { Treatments } \\
\text { WMM } \\
\text { WFF }\end{array}$} \\
\hline (1) & $\begin{array}{l}\text { Habitat and temporal effects: Community } \\
\text { structures differ between meadow and forest but not } \\
\text { between sampling time. Communities in meadow } \\
\text { exhibit phylogenetic clustering due to environmental } \\
\text { filtering resulting from disturbances, while forest } \\
\text { communities show phylogenetic overdispersion } \\
\text { resulting from niche partitioning in a relatively } \\
\text { stable environment. }\end{array}$ & & \\
\hline \multirow[t]{2}{*}{ (2) } & $\begin{array}{l}\text { Successional patterns: At early successional stages } \\
\text { drift predominates, resulting in a random pattern of } \\
\text { phylogenetic relatedness in defaunated blocks, while } \\
\text { selection drives communities to a deterministic }\end{array}$ & $\begin{array}{l}\text { M: Defaunated meadow soil blocks + } \\
\text { untreated meadow soil blocks (32) }\end{array}$ & $\begin{array}{l}\text { T1 - OMM } \\
\text { T2 - OMM } \\
\text { T3 - OMM } \\
\text { WMM (all time) }\end{array}$ \\
\hline & pattern at later successional stages. & $\begin{array}{l}\text { F: Defaunated forest soil blocks }+ \text { untreated } \\
\text { forest soil blocks }(40)\end{array}$ & $\begin{array}{l}\text { T1 - OFF } \\
\text { T2 - OFF } \\
\text { T3 - OFF } \\
\text { WFF (all time) }\end{array}$ \\
\hline (3) & $\begin{array}{l}\text { Soil origin effects: Successional patterns in } \\
\text { defaunated soil blocks differ between the transferred } \\
\text { soil blocks and the soil blocks installed in the } \\
\text { original habitat. }\end{array}$ & $\begin{array}{l}\text { OM: Defaunated soil blocks from different } \\
\text { origins surrounded by meadow }+ \text { untreated } \\
\text { meadow soil blocks ( } 32 \text { ) } \\
\text { OF: Defaunated soil blocks from different } \\
\text { origins surrounded by forest }+ \text { untreated } \\
\text { forest soil blocks ( } 44)\end{array}$ & $\begin{array}{l}\text { OFM* } \\
\text { OMM } \\
\text { WMM } \\
\text { OMF* } \\
\text { OFF } \\
\text { WFF }\end{array}$ \\
\hline \multirow[t]{2}{*}{ (4) } & $\begin{array}{l}\text { Indigenous species: Community structures in } \\
\text { untreated but transferred soil blocks change } \\
\text { gradually from those in soil blocks of the original } \\
\text { habitat to those in the transferred habitat. }\end{array}$ & $\begin{array}{l}\text { FM: Transferred forest soil blocks installed } \\
\text { in meadow }+ \text { untreated forest and meadow } \\
\text { soil blocks (49) }\end{array}$ & $\begin{array}{l}\text { WFF } \\
\text { WFM* } \\
\text { OFM } \\
\text { WMM }\end{array}$ \\
\hline & & $\begin{array}{l}\text { MF: Transferred meadow soil blocks } \\
\text { installed in forest }+ \text { untreated meadow and } \\
\text { forest soil blocks ( } 49 \text { ) }\end{array}$ & $\begin{array}{l}\text { WMM } \\
\text { WMF* } \\
\text { OMF } \\
\text { WFF }\end{array}$ \\
\hline
\end{tabular}




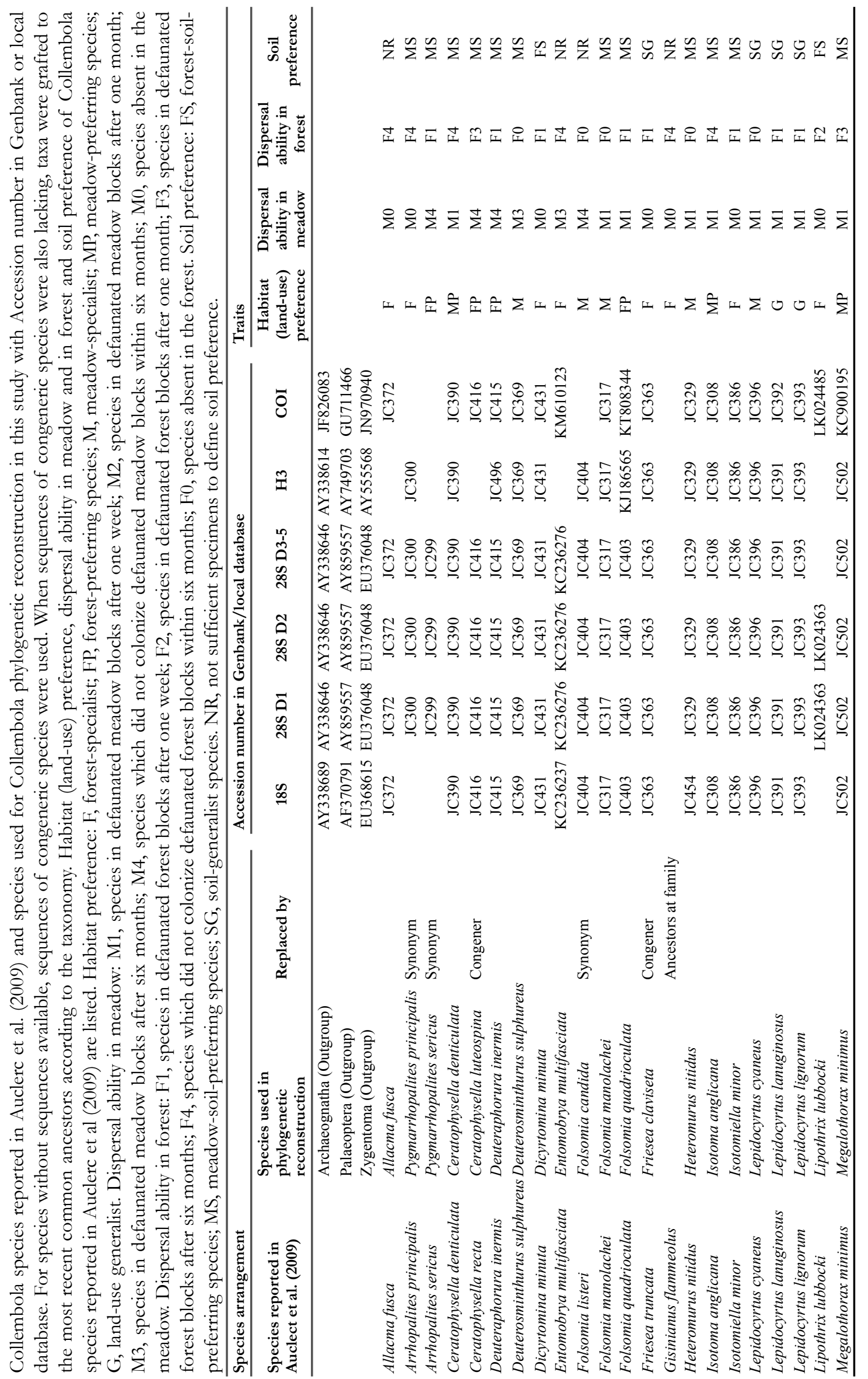




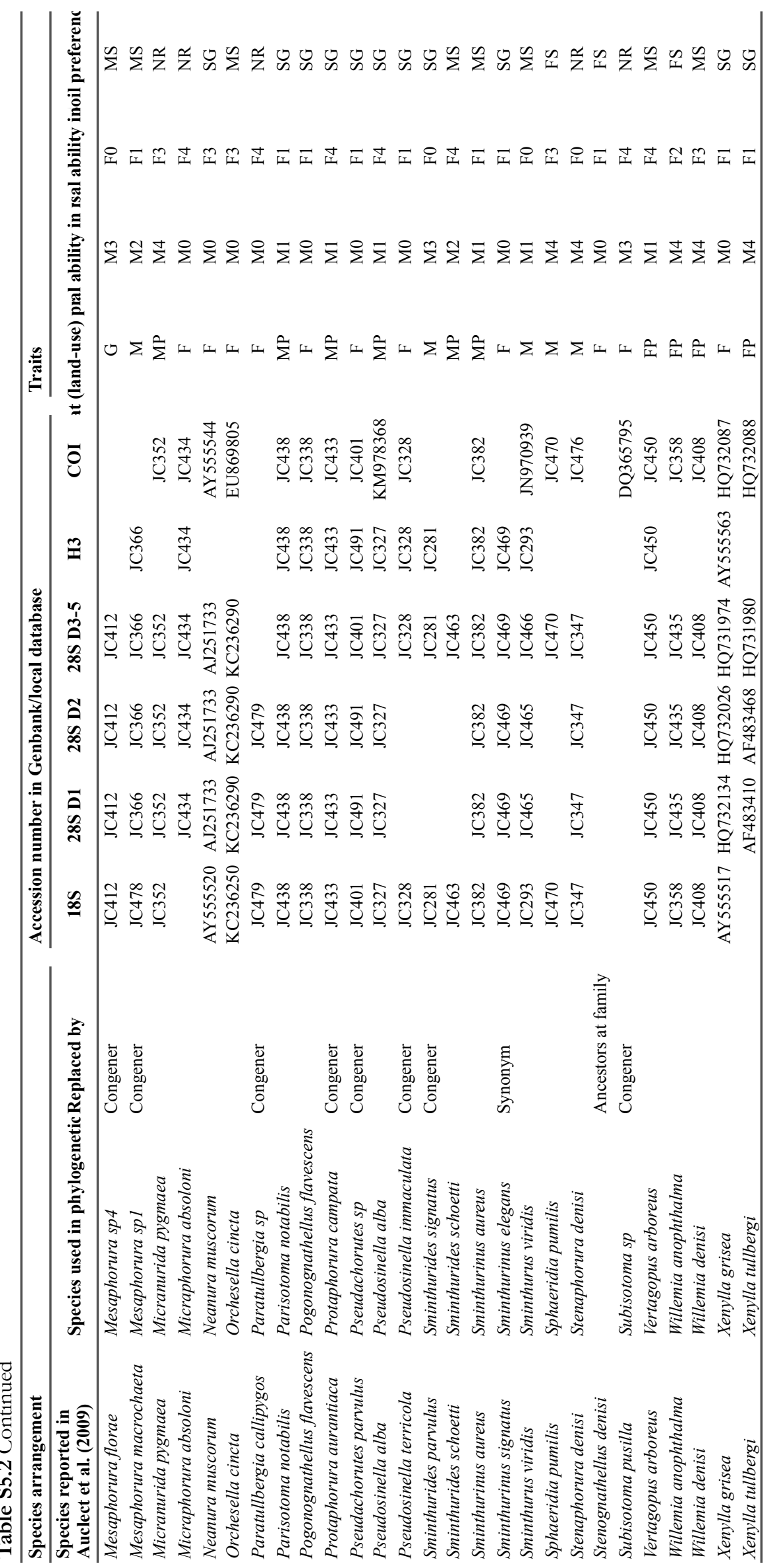




\section{Table S5.3}

Estimated number of transitions across 1,000 stochastic character mapping simulations of soil preferences of Collembola reported in Auclerc et al. (2009).

\begin{tabular}{|c|c|c|c|c|c|c|c|}
\hline \multirow[b]{2}{*}{ From: } & \multirow[t]{2}{*}{ To: } & \multicolumn{2}{|c|}{ Meadow-soil-preferring } & \multicolumn{2}{|c|}{ Soil-generalist } & \multicolumn{2}{|c|}{ Forest-soil-preferring } \\
\hline & & Median & Mean \pm SD & Median & Mean \pm SD & Median & Mean \pm SD \\
\hline Meadow-soil-preferring & & & & 8 & $7.6 \pm 2.0$ & 5 & $5.1 \pm 1.9$ \\
\hline Soil-generalist & & 3 & $4.2 \pm 3.4$ & & & 1 & $2.0 \pm 2.0$ \\
\hline Forest-soil-preferring & & 2 & $2.7 \pm 2.7$ & 1 & $1.9 \pm 2.0$ & & \\
\hline
\end{tabular}

\section{Table S5.4}

Estimated number of transitions across 1,000 stochastic character mapping simulations of dispersal ability in meadow of Collembola reported in Auclerc et al. (2009). Dispersal ability in meadow: M1, species in defaunated meadow blocks after one week; M2, species in defaunated meadow blocks after one month; M3, species in defaunated meadow blocks after six months; M4, species which did not colonize defaunated meadow blocks within six months; M0, species absent in the meadow.

\begin{tabular}{|c|c|c|c|c|c|c|c|c|c|c|c|c|c|c|}
\hline \multirow{2}{*}{$\begin{array}{l}\text { To: } \\
\text { From: }\end{array}$} & \multicolumn{3}{|c|}{ M1 } & \multicolumn{3}{|c|}{ M2 } & \multicolumn{3}{|c|}{ M3 } & \multicolumn{3}{|c|}{ M4 } & \multicolumn{2}{|r|}{ M0 } \\
\hline & Median & Mean \pm & SD & Median & Mean \pm & SD & Median & Mean \pm & SD & Median & Mean \pm & SD & Median & Mean \pm SD \\
\hline M1 & & & & $\mathbf{0}$ & $0.5 \pm$ & 0.9 & 2 & $2.0 \pm$ & 1.9 & 2 & $2.9 \pm$ & 3.0 & 9 & $10.1 \pm 5.6$ \\
\hline M2 & 0 & $0.4 \pm$ & 0.9 & & & & 1 & $1.5 \pm$ & 1.5 & 0 & $0.6 \pm$ & 1.2 & 1 & $1.1 \pm 1.7$ \\
\hline M3 & $\mathbf{0}$ & $1.0 \pm$ & 1.7 & 1 & $1.4 \pm$ & 1.2 & & & & 1 & $1.3 \pm$ & 1.9 & 1 & $1.4 \pm 2.2$ \\
\hline M4 & 2 & $2.7 \pm$ & 2.9 & $\mathbf{0}$ & $0.8 \pm$ & 1.2 & 2 & $2.1 \pm$ & 2.0 & & & & 8 & $9.2 \pm 6.7$ \\
\hline M0 & 8 & $8.5 \pm$ & 4.8 & 1 & $1.3 \pm$ & 1.5 & 2 & $2.1 \pm$ & 2.1 & 8 & $9.1 \pm$ & 5.3 & & \\
\hline
\end{tabular}




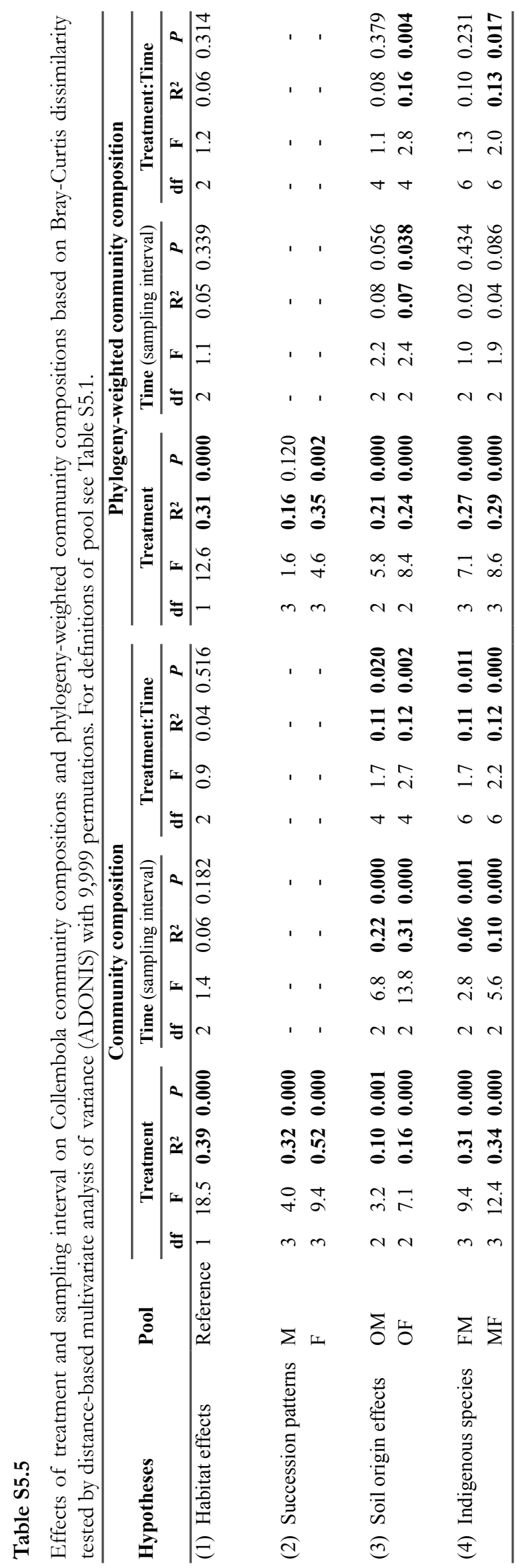




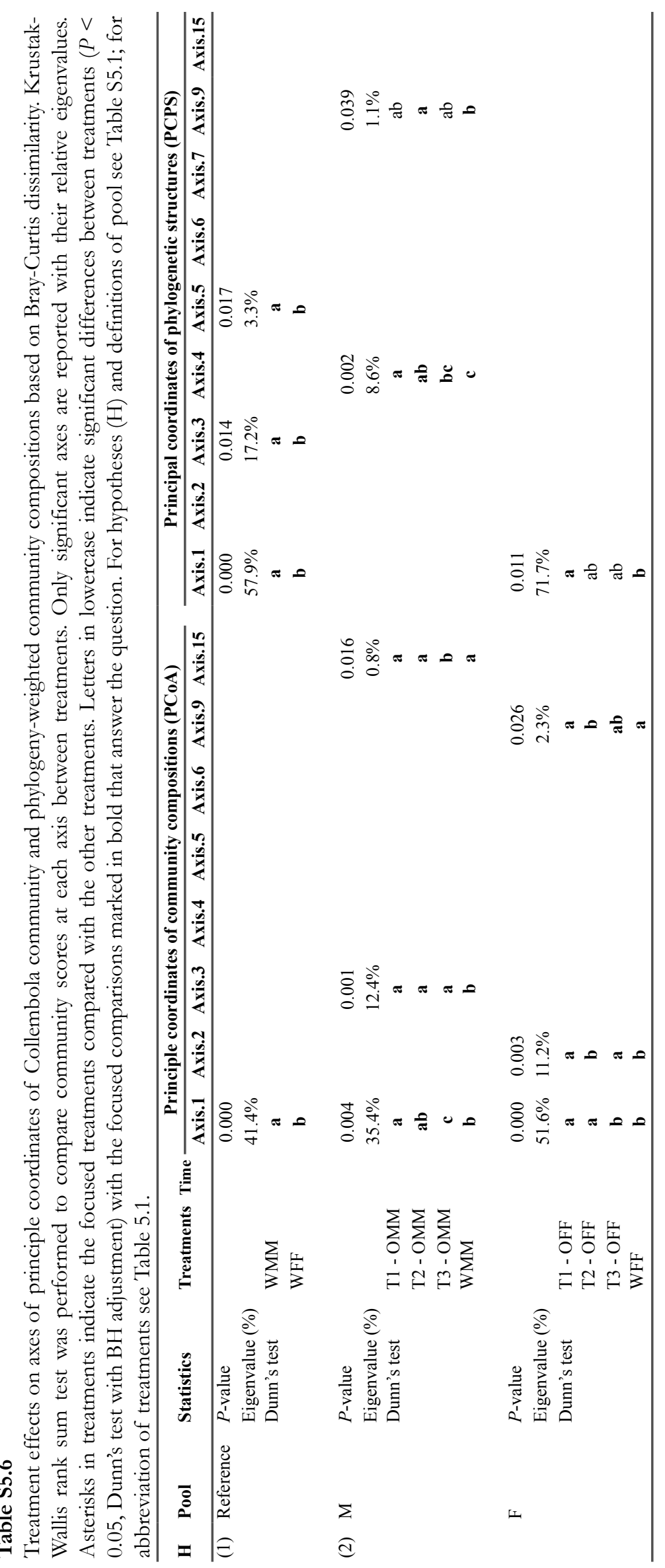




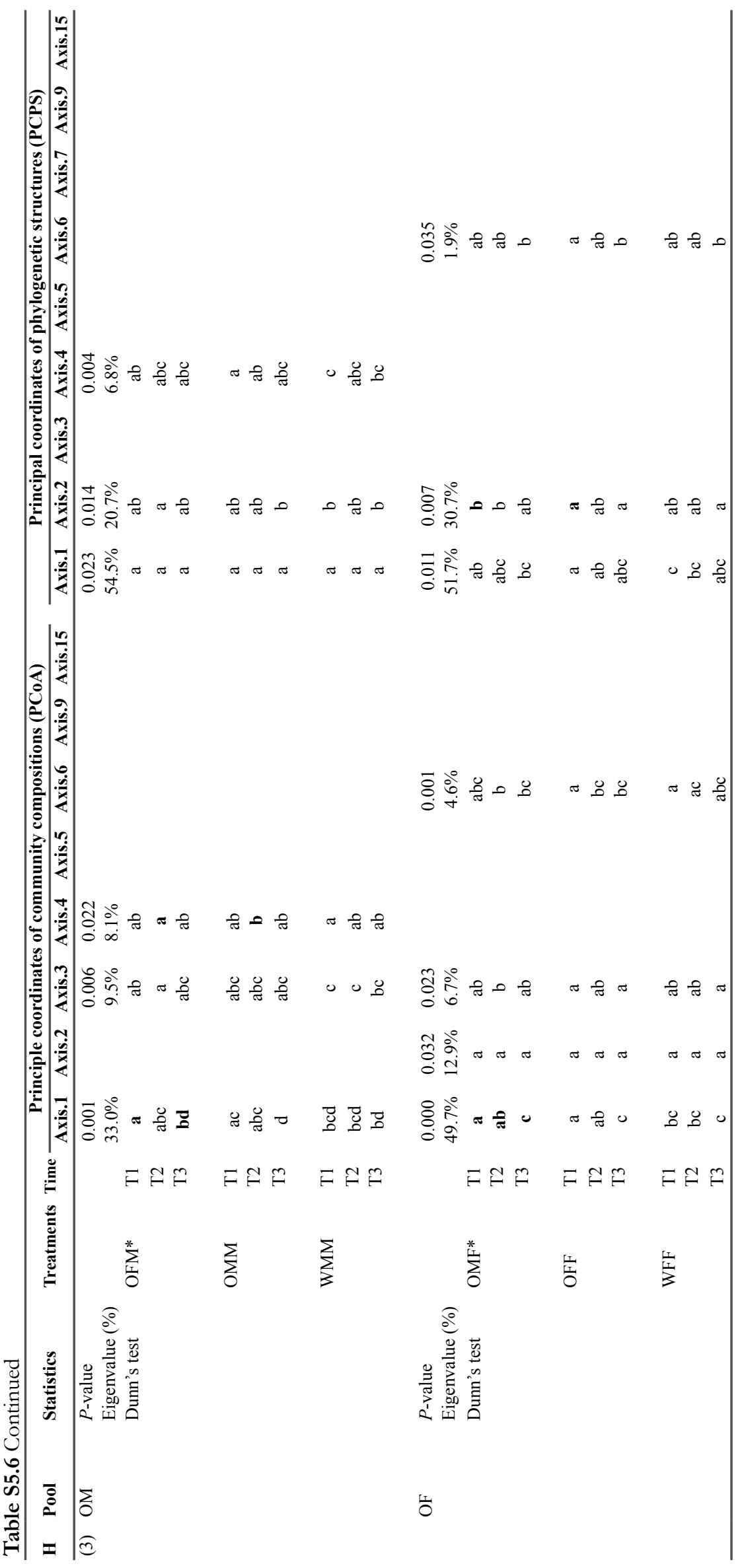




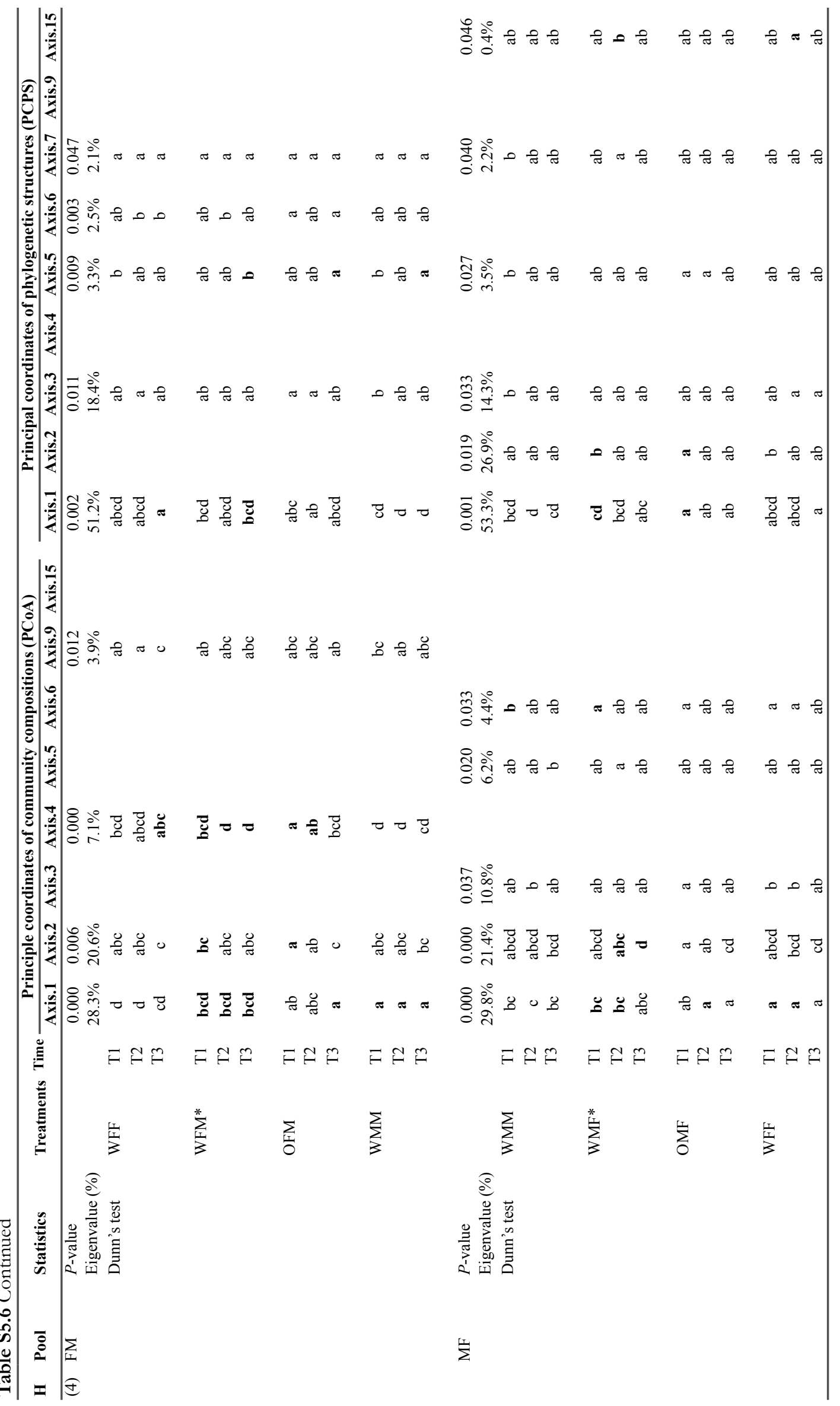




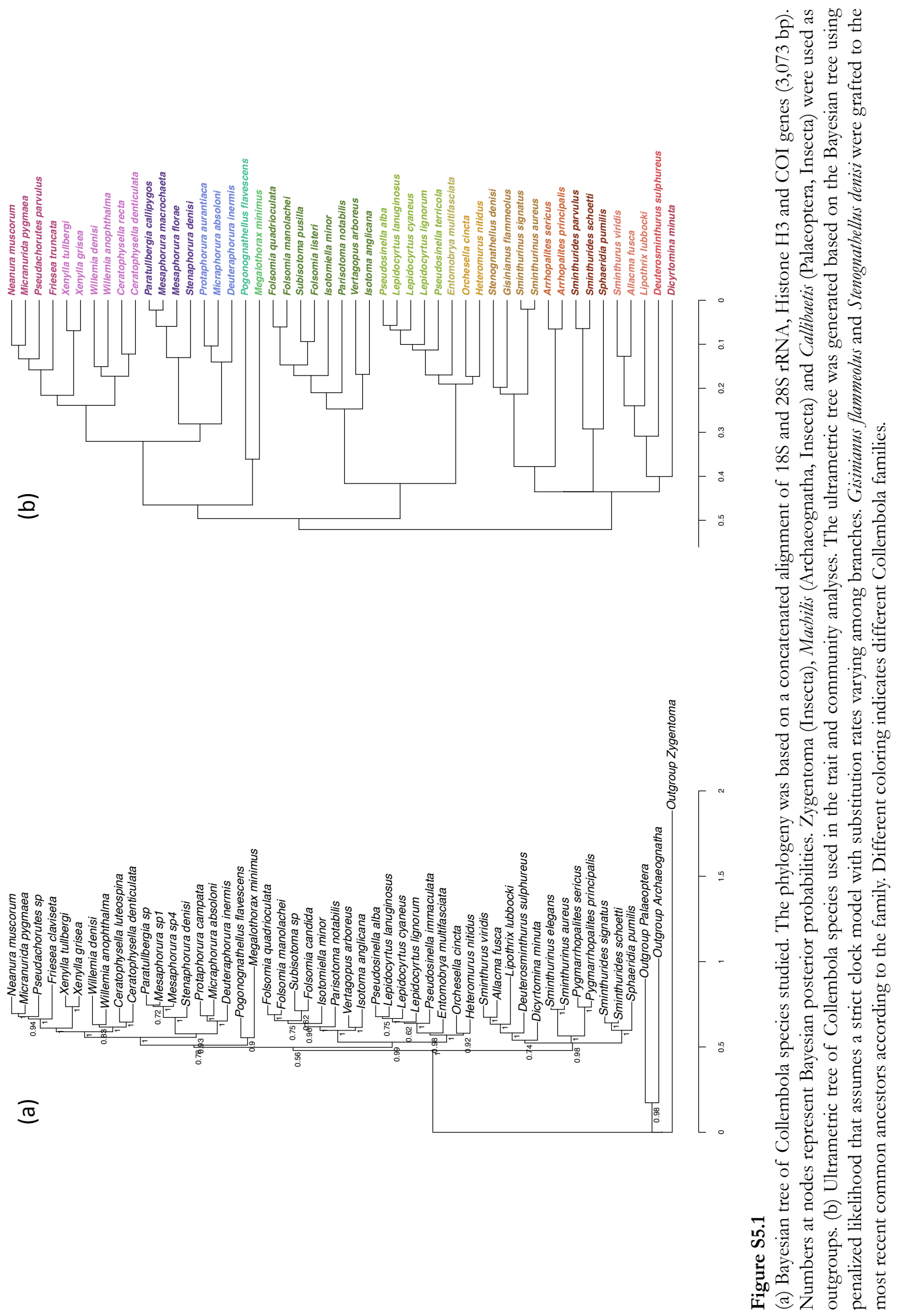




\section{Chapter 6}

\section{General Discussion}

This thesis is the first attempt to apply phylogenetic approaches to elucidate assembly processes of soil Collembola communities. By integrating community phylogenetic approaches with phylogenetic comparative and trait-based methods, this thesis presents major advances in understanding biodiversity of soil animals from both ecological and evolutionary perspectives. The conceptual model proposed in Chapter 1 (Figure 1.1) can be applied to any other soil taxon and thus provides a framework for future studies.

In this thesis, I reconstructed the evolutionary history of several traits in Collembola, including morphological characters (Chapter 2), ecological preferences (Chapters 2 and 5), physiological attributes and food resources (Chapter 3). Phylogenetic relatedness and trait similarity of species within local communities ( $\alpha$-diversity) were used to infer assembly processes, given the assumption of phylogenetic signal in ecologically relevant traits. A phylogenetic $\beta$-diversity approach was used to elucidate community-environmental associations from an evolutionary perspective (Chapters 4 and 5). Furthermore, the community phylogenetic approaches were applied to both field observational (Chapter 4) and experimental (Chapter 5) studies.

In the following sections, I first ascribe the patterns of Collembola communities found in previous chapters to the scenarios in the conceptual model (Figure 1.1) by specifically exploring trait evolution, trait similarity and phylogenetic relatedness between coexisting species, then discuss the likely processes referring to the four high-level processes, selection, dispersal, drift and speciation, proposed in The Theory of Ecological Communities (Vellend 2010, 2016). Finally, I supply a roadmap for soil ecologists to integrate phylogenetic comparative methods, community phylogenetic analyses and trait-based approaches in studies on the assembly processes of soil communities.

\section{Assembly processes of soil Collembola communities}

\section{Disturbance as a selecting factor}

Phylogenetic clustering is revealed in the abundant Collembola species inhabiting arable fields near Göttingen (Chapter 4), following scenario (a) in which environmental filtering is a predominant process, if the traits underlying community assembly processes are conserved relative to the ancestor and thus exhibit phylogenetic signal, or scenario (c) in which niche partitioning structures communities, if the niche traits diverged from the ancestral state (Figure 1.1). Traits such as body length, vertical stratification, pigmentation, number of ommatidia and reproductive mode are similar between phylogenetically related species and evolution of body shape was constrained (Chapter 2), suggesting that scenario (a) is more likely. As a logical consequence, traits between coexisting species should be more similar than that predicted by the null model. However, randomness in trait similarity suggests that while 
some of the examined traits are filtered by the environment, other traits are likely driven by other factors from the opposite direction. For example, niche partitioning may structure coexisting species which differed in traits, such as vertical stratification, pigmentation, number of ommatidia and reproductive mode, that co-evolved during Collembola diversification, and thus likely diminish the effect of environmental filtering, shifting the traits from similar to random patterns. Nevertheless, phylogenetic clustering in Collembola communities collected from arable fields is consistent with patterns in other taxa inhabiting disturbed environments (Ding et al. 2012, Pellissier et al. 2013, Gianuca et al. 2014) and with findings from trait-based analysis of Collembola communities inhabiting salt marshes (Widenfalk et al. 2015). Overall, environmental filtering is likely the predominant process in soil animal communities in habitats where disturbance may result in homogeneity of soil properties (Maaß et al. 2014).

Phylogenetic overdispersion is found in the forest Collembola communities collected in Göttingen (Chapter 4). Again, traits between coexisting species show a random pattern. Collembola communities in forests thus follow scenario (b) or (d) (Figure 1.1), depending on how the process-relevant traits have evolved and how they are structured by different processes. If the traits show phylogenetic signal, coexistence of distantly related species may result in different traits in local communities. Randomness in trait patterns, thus suggests that environmental filtering may still work but with limited influence, while niche partitioning is the predominant process working on Collembola living in forest soils. High spatial heterogeneity in forest soils likely results in different traits of communities (Maaß et al. 2014, Widenfalk et al. 2016). Also, soil food webs in stable habitats such as forests are complex (Scheu and Falca 2000, Digel et al. 2014). Given that Collembola species span several trophic levels (Chahartaghi et al. 2005, Pollierer et al. 2009) with taxonomically related species occupying similar trophic levels (Potapov et al. 2016), communities comprised of species from a variety of phylogenetic clades may be able to exploit a wide range of food resources and thus facilitate coexistence in forests. Overall, niche partitioning in micro-habitats and food resources is likely a predominant process in Collembola communities in stable habitats, although environmental filtering working on phylogenetically convergent traits may produce similar patterns.

The general hypothesis that Collembola communities in disturbed habitats are determined by environmental filtering, while in relatively stable habitats interspecific competition/niche partitioning is predominant, is supported by phylogenetic relatedness but not by trait similarity (Chapter 4), even though the tested traits exhibit phylogenetic signal (Chapter 2). In contrast, Collembola communities in Morvan Regional Natural Park (Chapter 5, Hypothesis 1) show phylogenetic clustering in forest but overdispersion in meadow habitats. Discrepancies between the two studies may be due to the sampling design. In Chapter 4, Collembola communities were sampled from replicated forest sites and grasslands, while in Chapter $\mathbf{5}$ communities of each type of habitat were replicated by soil blocks from a single site. Assembly processes of Collembola likely differ not only between habitats but may also be site-specific. Furthermore, successional stages of communities may also influence relative strengths of the forces driving community assembly, as demonstrated in Chapter 5 (Hypothesis 2) in which niche partitioning 
becomes stronger at later successional stages and may balance the effects of environmental filtering. Overall, the results of this thesis indicate that Collembola communities are structured by selection processes such as environmental filtering and niche partitioning, which vary in different habitats and with different successional stages.

\section{Dispersal as a high-level process}

Data on successional trajectories in the manipulative experiment (Auclerc et al. 2009; Chapter 5) show that species dispersal, interacting with selection processes, determine community composition of Collembola. Results indicate that soil properties of the defaunated blocks influence community assembly of Collembola at initial stages of succession, while at later stages community assembly is dominated by mass effects due to continuous immigration of species from the surrounding habitat (Hypothesis 3). As a consequence of dispersal (dispersal as a high-level process), community compositions change gradually from those resembling the original habitats to those of the new habitats (Hypothesis 4). These results are consistent with previous findings at different spatial scales, from plot (Åström and Bengtsson 2011) to landscape (Ingimarsdóttir et al. 2012), reemphasizing the importance of mass effects (i.e., consequences of dispersal) on Collembola community compositions. Collembola, considered a fast disperser among the soil animals (but see Ojala and Huhta 2001), can disperse actively at small spatial scales (Bengtsson et al. 1994) but may also be transmitted via other vectors over long geographical distances (Costa et al. 2013). Furthermore, dispersal ability of Collembola is likely related to their life forms, such as surface-living (epedaphic) or soil-dwelling (euedaphic) (Bengtsson et al. 1994, Hågvar 2000, Ojala and Huhta 2001, Zhang et al. 2017), and to food availability and quality in the habitats (Bengtsson et al. 1994, Stötefeld et al. 2012).

\section{Stochasticity in communities-ecological drift}

One prediction of The Theory of Ecological Communities (Vellend 2010, 2016) is that the signature of ecological drift as a high-level process on community assembly is random patterns of traits. In Chapter 4, Collembola collected from the grasslands and those dwelling in soil (euedaphic) exhibit random patterns in both trait similarity and phylogenetic relatedness. Considering that disturbance and spatial heterogeneity in grasslands are between that in the arable fields and forests, it may be that communities in moderately disturbed habitats are driven by environmental filtering and niche partitioning with similar strengths, therefore resulting in the random patterns. Interestingly, euedaphic Collembola are also likely influenced by the stochastic processes, irrespective of the habitat types. Whether ecological drift plays a major role in the coexistence of deep soil species needs further investigations. Although some have attempted to evaluate the importance of stochasticity in community assembly of Collembola (Ims et al. 2004, Ingimarsdóttir et al. 2012, Chen et al. 2015, Sha et al. 2015), to the best of my knowledge, no study so far explicitly tests or quantifies the contribution of drift (i.e., demographic stochasticity) to community 
assembly in soil, presumably due to the difficulty to discriminate between dispersal and demographic stochasticity in the spatial processes.

\section{Speciation-with emphasis on trait evolution}

\section{Phylogenetic reconstruction of Collembola}

Results of phylogenetic inference of Collembola species indicate that Entomobryoidea and Isotomidae diverged earlier from the other Collembola lineages, while Poduromorpha and Symphypleona are sister groups (Chapter 2), contrary to the previous studies which recover Symphypleona diverging earlier than the other Collembola (D’Haese 2002, Xiong et al. 2008, Schneider et al. 2011, Yu et al. 2016). Although marker selection, unbalanced taxon sampling and choice of outgroup taxa may affect the topology of phylogenetic trees (Heath et al. 2008, Rosenfeld et al. 2012), genetic markers ranging from conserved (18S rRNA, H3) to variable (28S rRNA, COI) are used in this study to generate a phylogenetic tree for 102 locally occurring Collembola species including 51 genera from 18 families. Thus, this tree provides the most comprehensive Collembola phylogeny to date. Future studies applying phylogenomic methods may help to resolve phylogenetic relationships between basal Collembola lineages (van Straalen et al. 2008, Misof et al. 2014, Carapelli et al. 2014).

\section{Trait evolution in light of species coexistence}

According to the $\alpha$ and $\beta$ niche traits concept, species within a community possess both similar and different traits. Similar traits related to $\beta$ niches allow community members to cope with certain environmental conditions, while different $\alpha$ niche traits avoid competition for resources (Silvertown et al. 2006). Since traits are evolutionary signatures of species diversification, these two types of traits may evolve in different ways, with $\beta$ niche traits usually phylogenetically conserved, and $\alpha$ niche traits evolutionarily labile (Silvertown et al. 2006; Ackerly et al. 2006; Best and Stachowicz 2013).

Results of Chapter 2 demonstrate that body shape of Collembola evolved quickly early in diversification but followed by relative stasis, presumably under stable evolutionary or ecological constraints. Pigmentation, number of ommatidia and reproductive mode of Collembola were all associated with vertical stratification during species diversification. Although ancestral traits of Collembola were likely slender body, hemiedaphic way of life, sexual reproduction, possession of many ommatidia and bright color, these traits may have changed several times during diversification of species. Overall, the traits considered in this chapter may be categorized as $\beta$ niche traits, since they are more or less related to species adaptation to the abiotic conditions. Interestingly, in each phylogenetic clade, there is one or a few Collembola species with high abundance in the Göttingen region, resulting in lower phylogenetic signal measured in species total abundance compared to that predicted by Brownian motion model. Since abundance of soil microarthropods positively correlates with the amount of food resources 
available in the habitats (Domes et al. 2007, Chahartaghi et al. 2009), food resource exploitation of Collembola might have evolved convergently during species diversification.

In Chapter 3, neutral lipid fatty acid composition is proposed as a functional trait that relates to both physiological functions and food resources of Collembola. Long-chain polyunsaturated fatty acids, related to physiological functions, demonstrate phylogenetic signal, while most food resource biomarker fatty acids and the ratios between bacterial, fungal and plant biomarker fatty acids exhibit no phylogenetic signal. Presumably, species with close phylogenetic affinity experienced similar environments during diversification, while divergence in exploitation of food resources among closely related species may favor species coexistence. Since stable isotope data indicates phylogenetic (taxonomic) conservatism in Collembola trophic niches (Potapov et al. 2016) and fatty acid composition complements stable isotopes in analyzing trophic niche of soil animals (Ferlian et al. 2015), Collembola feeding traits are, on one hand, likely to relate to the evolutionary history of species; on the other hand, they may retain variability to reduce competition. More data on trophic niches and food resources identified indirectly by stable isotope signature and neutral lipid fatty acids and directly by gut content and microbiome analyses in various species from different phylogenetic groups are needed to test this hypothesis.

Dispersal ability is a composite trait. Chapter 5 finds close Collembola relatives colonizing defaunated soil blocks with similar speed in a meadow habitat. However, species abilities to immigrate from the surrounding habitat and survive in a new habitat are determined not only by morphological characters, such as legs, antennae, furca and visual apparatus (Ponge et al. 2006, Auclerc et al. 2009), but also by physiological attributes such as moisture tolerance (Kuznetsova 2003) and presumably by food resource quality (Bengtsson et al. 1994, Stötefeld et al. 2012). Therefore, dispersal ability ascribed by Auclerc et al. (2009) and subsequently analyzed in this chapter should be considered as a summarized response, and phylogenetic signal measured in dispersal ability likely reflects phylogenetic niche conservatism in other functional traits. Future studies may estimate dispersal ability of different species using observations (Zhang et al. 2017) and genetic tools (van der Wurff et al. 2003) and further test phylogenetic signal in dispersal ability.

Except for the fatty acid composition, this thesis mainly relies on the trait data compiled from literature at species level. The situation is probably applicable to most soil animals where information on field-measured traits at individual or population levels is still lacking (Pey et al. 2014, Moretti et al. 2017). To understand intraspecific variations, there remains a need to directly measure field-derived individuals including different cohorts and populations. Furthermore, laboratory measurements of functional traits of soil animals may help to determine the range of species fundamental niches, which may be subsequently compared with field observation data to reveal the differences in realized niches under the influences of other coexisting species. Explicitly measuring individual properties using both laboratory experiments and field observations for multiple traits, including morphological, physiological and trophic attributes, may help uncover the mechanisms driving and maintaining species coexistence in soil animals and thus explain the enigma of soil animal biodiversity (Anderson 1975). 


\section{A roadmap for implementation}

In this thesis I use phylogeny as a surrogate for traits, a technique that has been much debated recently among plant ecologists (Gerhold et al. 2015, de Bello et al. 2015, Šímová 2016, Prinzing 2016, Rosado et al. 2016, McPeek 2017). One main critique is that traits are the objects that relate to or underlie different processes, not phylogeny. Phylogeny should not be used to infer assembly processes without considering the many assumptions behind it (Gerhold et al. 2015, McPeek 2017). For example, traits underlying processes must be identified and included in the null model tests; the assumption of phylogenetic conservatism of traits must be validated; phylogenetic dispersion should reflect trait dispersion; similarity in traits should translate to interspecific competition; competition should lead to species exclusion and so on. These many assumptions have received very limited empirical support (Gerhold et al. 2015, Prinzing 2016). However, phylogeny may show its advantage as a representation of multiple unmeasurable traits (Cadotte et al. 2013, de Bello et al. 2015). Phylogeny may therefore be treated as an independent variable and combined with existing trait data in the case in which the process-related traits are phylogenetically convergent or labile (Cadotte et al. 2013). Furthermore, beyond its use as a surrogate for traits, phylogenetic information can be used for studies on adaptation of species in trait-based approaches (de Bello et al. 2015) and for asking evolutionary questions of community assembly (Gerhold et al. 2015, Prinzing 2016).

For most soil animal species, however, except for morphological traits, we lack information on other aspects of traits, such as physiological attributes or those related to food resources. The situation may be worse for rare species and those difficult to culture in the laboratory. Also, species possess multiple traits that may correlate statistically or evolutionarily. Therefore, as compared to measuring all possible relevant traits, phylogenetic relationships may be a more pragmatic approach to the problem, considering the current developing stage of trait databases of soil animals, although the same concerns raised by plant ecologists may likely also apply to soil animals. Nevertheless, when trait information is lacking, we may use phylogeny to develop a first impression of the likely distribution of species traits in the communities, from which we are able to further study ecological and evolutionary hypotheses of species coexistence in soil (Cadotte et al. 2013, Gerhold et al. 2015, de Bello et al. 2015; but see Rosado et al. 2016). To that end, I propose the following roadmap for analytical strategies to study community assembly of soil animals using phylogenetic information and functional traits (Figure 6.1):

1. Propose traits which are likely relevant for community assembly processes. Multiple traits of soil animals from various aspects need to be considered, including morphological characters, physiological attributes and those related to food resources. Dispersal ability and ecological preference of species may also be considered as traits. Here we may ask: Are these traits measurable for all species (Q1)? If traits underlying community assembly processes are known and measurable for all species, we then go on with step 2. 
2. Measure traits for individuals/populations in the laboratory/field. Traits should be measured at individual, population or species levels. Both laboratory experiments and field observations are needed to achieve species fundamental and realized niches. We may ask: Do trait variations reflect processes (Q2)? For example, whether environmental gradients correlate with community-weighted mean traits (Widenfalk et al. 2015), or whether coexisting species possess similar traits. Here, ecological processes, usually selection processes, can be investigated using trait-based approaches (the red route; I). However, in a situation where no functional traits are known, or traits of rare species are difficult to measure (answer no to Q1), we need to go on with step 2 a.

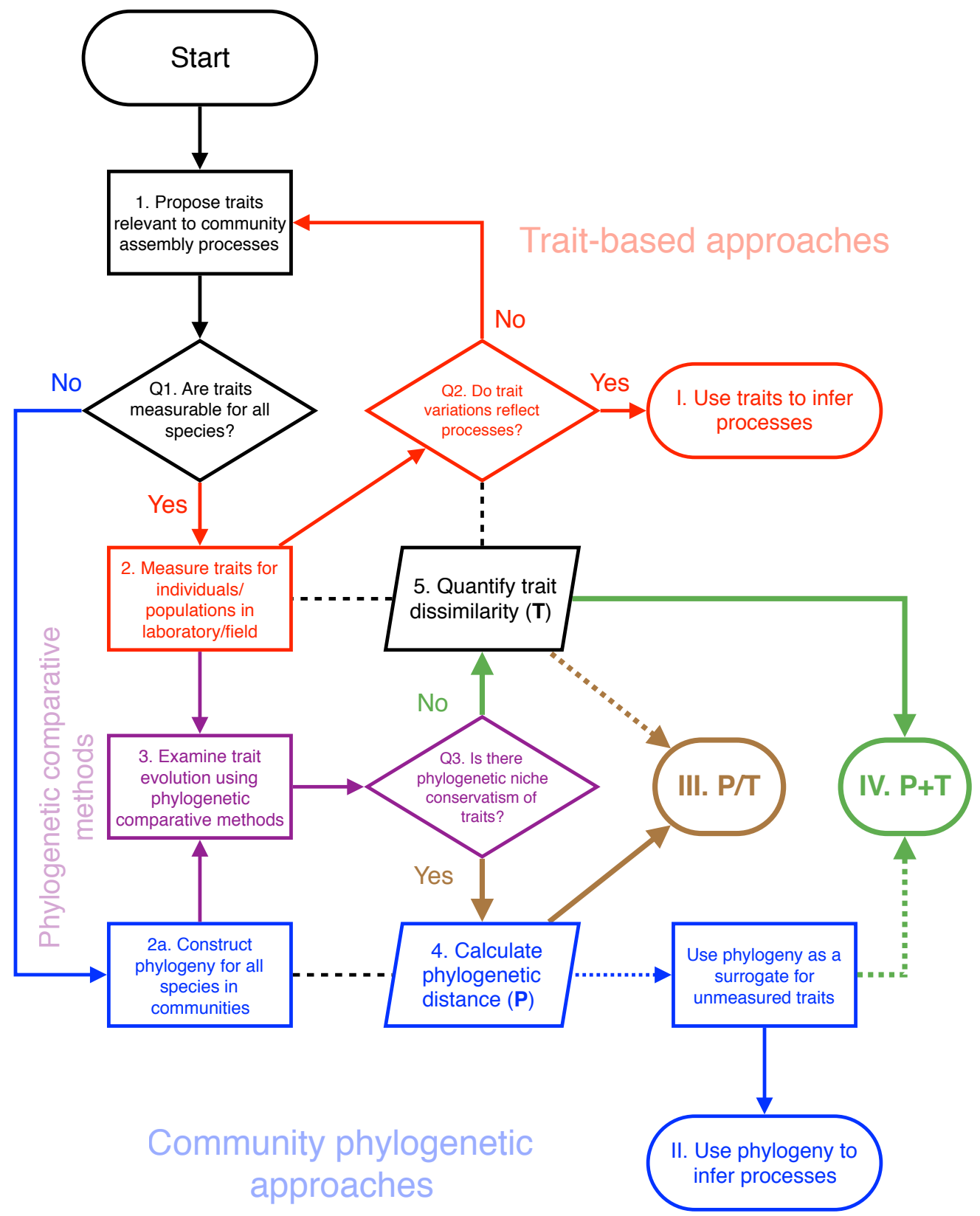

Figure 6.1

A guideline for analytical strategies to study community assembly of soil animals using phylogenetic information and functional traits. For details see the text. 
2a. Construct phylogeny for all species in communities. We may take phylogeny as a surrogate for the unknown or unmeasured traits, assuming phylogenetic signal (de Bello et al. 2015), and get first insight into the phylogenetic (thus likely trait) structures in local communities (the blue route). In this situation, phylogeny provides an alternative tool to quantify differences between species, without a priori knowledge of traits (II). This approach may also apply to communities consisting of cryptic species that are unable to be differentiated by any morphological characters, a situation frequently occurring in soil animals (Zhang et al. in revision), in which only genetic distances between cryptic species are known. In such a case phylogeny shows its advantage to quantifying differences between (cryptic) species. Furthermore, from these patterns, other hypotheses may be proposed; for example, if phylogenetic clustering is found, we may hypothesize that some traits do differ between coexisting species which allow coexistence, and predict what these traits may be. This method is adopted, for example, in Chapter $\mathbf{5}$ of this thesis.

3. Examine trait evolution. Then, having species trait data and their phylogenetic tree, we may investigate evolutionary processes that shape trait variations in different species (the purple route). Trait evolution can be analyzed using phylogenetic comparative methods, such as phylogenetic signal measurements, model tests and ancestral state estimation as demonstrated in Chapters 2 and 3. Taxonomic or phylogenetic scales should be considered in the analyses. Here, the question we may ask includes: Is there phylogenetic niche conservatism of the traits (Q3)? Different answers lead to different ways to the subsequent analyses: If yes, first go to step 4 and then consider step 5; if no, go to step 5 and combine this with step 4.

4. Calculate phylogenetic distances, and 5. quantify trait dissimilarity between species. If traits are phylogenetically conserved or follow Brownian motion model of evolution (the brown route), phylogenetic distance matrix $(\mathbf{P})$ is calculated and used to infer processes. At the same time, the trait dissimilarity matrix $(\mathbf{T})$ is quantified to evaluate whether phylogenetic relatedness and trait similarity give congruent results (III; P/ $\mathbf{T}$ ), as demonstrated in Chapter 4. On the contrary, if traits evolved randomly or convergently (the green route), the trait distance matrix $(\mathbf{T})$ should be treated as an independent dataset which can be combined with the phylogenetic distance matrix $(\mathbf{P})$ to evaluate the relative contributions of each dataset $(\mathbf{P}$ and $\mathbf{T})$ to the differences between community members (IV; P+T; Cadotte et al. 2013, Gong et al. submitted).

\section{Conclusions}

This thesis provides the first example of integrating phylogenetic comparative methods, community phylogenetic analyses and trait-based approaches in studies on the assembly processes of soil invertebrates using both field observations and experimental manipulations with Collembola as the model soil animal group. Phylogenetic signal in process-related functional traits is demonstrated by using comparative methods for body shape, body length, pigmentation, number of ommatidia, reproductive mode and vertical stratification that reflect adaptations to the environment. For the first time, neutral lipid fatty acid composition is established as a functional trait related to both food resources and physiological 
attributes of species. Phylogenetic signal in fatty acid composition suggests that species with close phylogenetic affinity experienced similar environments during divergence, while niche partitioning in food resources favored species coexistence. Furthermore, differences in phylogenetic relatedness and trait similarity of local communities provide hints on assembly processes, i.e., Collembola in arable fields are mainly structured by environmental filtering, while niche partitioning dominates in forests. In addition, the relative importance of these mechanisms varies between soil strata and between phylogenetic lineages. Furthermore, combining of field manipulative experiments with community phylogenetic approaches allows deeper understanding of assembly processes in Collembola communities along successional trajectories in different habitats. Both the conceptual model and analytical roadmap proposed in this thesis can be applied to other soil taxa. Future studies integrating different approaches may shed new light on the mechanisms driving and maintaining species coexistence and biodiversity in soil.

\section{References}

Ackerly, D.D., Schwilk, D.W. \& Webb, C.O. (2006) Niche evolution and adaptive radiation: testing the order of trait divergence. Ecology, 87, S50-61.

Anderson, J.M. (1975) The enigma of soil animal species diversity. Progress in Soil Zoology: Proceedings of the 5th International Colloquium on Soil Zoology (ed J. Vaněk), pp. 51-58. Springer Netherlands, Dordrecht.

Åström, J. \& Bengtsson, J. (2011) Patch size matters more than dispersal distance in a mainland-island metacommunity. Oecologia, 167, 747-757.

Auclerc, A., Ponge, J.-F., Barot, S. \& Dubs, F. (2009) Experimental assessment of habitat preference and dispersal ability of soil springtails. Soil Biology and Biochemistry, 41, 1596-1604.

de Bello, F., Berg, M.P., Dias, A.T.C., Diniz-Filho, J.A.F., Götzenberger, L., Hortal, J., Ladle, R.J. \& Lepš, J. (2015) On the need for phylogenetic "corrections" in functional trait-based approaches. Folia Geobotanica, 50, 349-357.

Bengtsson, G., Hedlund, K. \& Rundgren, S. (1994) Food- and density-dependent dispersal: evidence from a soil collembolan. Journal of Animal Ecology, 63, 513-520.

Best, R.J. \& Stachowicz, J.J. (2013) Phylogeny as a proxy for ecology in seagrass amphipods: which traits are most conserved? PLOS ONE, 8, e57550.

Cadotte, M., Albert, C.H. \& Walker, S.C. (2013) The ecology of differences: assessing community assembly with trait and evolutionary distances. Ecology Letters, 16, 1234-1244.

Carapelli, A., Convey, P., Nardi, F. \& Frati, F. (2014) The mitochondrial genome of the antarctic springtail Folsomotoma octooculata (Hexapoda; Collembola), and an update on the phylogeny of collembolan lineages based on mitogenomic data. Entomologia, 2.

Chahartaghi, M., Langel, R., Scheu, S. \& Ruess, L. (2005) Feeding guilds in Collembola based on nitrogen stable isotope ratios. Soil Biology and Biochemistry, 37, 1718-1725.

Chahartaghi, M., Maraun, M., Scheu, S. \& Domes, K. (2009) Resource depletion and colonization: A comparison between parthenogenetic and sexual Collembola species. Pedobiologia, 52, 181-189.

Chen, Y., Amundrud, S.L. \& Srivastava, D.S. (2015) Spatial variance in soil microarthropod communities: Niche, neutrality, or stochasticity? Ecoscience, 21, 1-14.

Costa, D., Timmermans, M.J.T.N., Sousa, J.P., Ribeiro, R., Roelofs, D. \& van Straalen, N.M. (2013) Genetic structure of soil invertebrate populations: Collembolans, earthworms and isopods. Applied Soil Ecology, 68, 61-66. 
D'Haese, C.A. (2002) Were the first springtails semi-aquatic? A phylogenetic approach by means of 28S rDNA and optimization alignment. Proceedings of the Royal Society of London B, 269, 1143-1151.

Digel, C., Curtsdotter, A., Riede, J., Klarner, B. \& Brose, U. (2014) Unravelling the complex structure of forest soil food webs: higher omnivory and more trophic levels. Oikos, 123, 1157-1172.

Ding, Y., Zang, R., Letcher, S.G., Liu, S. \& He, F. (2012) Disturbance regime changes the trait distribution, phylogenetic structure and community assembly of tropical rain forests. Oikos, 121, 1263-1270.

Domes, K., Scheu, S. \& Maraun, M. (2007) Resources and sex: Soil re-colonization by sexual and parthenogenetic oribatid mites. Pedobiologia, 51, 1-11.

Ferlian, O., Klarner, B., Langeneckert, A.E. \& Scheu, S. (2015) Trophic niche differentiation and utilisation of food resources in collembolans based on complementary analyses of fatty acids and stable isotopes. Soil Biology and Biochemistry, 82, 28-35.

Gerhold, P., Cahill, J.F., Winter, M., Bartish, I. V \& Prinzing, A. (2015) Phylogenetic patterns are not proxies of community assembly mechanisms (they are far better). Functional Ecology, 29, 600-614.

Gianuca, A.T., Dias, R.A., Debastiani, V.J. \& Duarte, L.D.S. (2014) Habitat filtering influences the phylogenetic structure of avian communities across a coastal gradient in southern Brazil. Austral Ecology, 39, 29-38.

Gong, X., Chen, T.-W., Zieger, S., Bluhm, C., Heidemann, K., Schaefer, I., Maraun, M., Liu, M. \& Scheu, S. Phylogenetic and trophic determinants of gut microbiota in soil detritivores. Submitted.

Hågvar, S. (2000) Navigation and behaviour of four Collembola species migrating on the snow surface. Pedobiologia, 44, 221-233.

Heath, T.A., Hedtke, S.M. \& Hillis, D.M. (2008) Taxon sampling and the accuracy of phylogenetic analyses. Journal of Systematics and Evolution, 46, 239-257.

Ims, R.A., Leinaas, H.P. \& Coulson, S. (2004) Spatial and temporal variation in patch occupancy and population density in a model system of an arctic Collembola species assemblage. Oikos, 105, 89-100.

Ingimarsdóttir, M., Caruso, T., Ripa, J., Magnúsdóttir, Ó.B., Migliorini, M. \& Hedlund, K. (2012) Primary assembly of soil communities: disentangling the effect of dispersal and local environment. Oecologia, 170, 745-754.

Kuznetsova, N. (2003) Humidity and distribution of springtails. Entomological Review, 83, 230-238.

Maaß, S., Migliorini, M., Rillig, M.C. \& Caruso, T. (2014) Disturbance, neutral theory, and patterns of beta diversity in soil communities. Ecology and Evolution, 4, 4766-4774.

McPeek, M.A. (2017) Evolutionary Community Ecology. Princeton University Press.

Misof, B., Liu, S., Meusemann, K., Peters, R.S., Donath, A., Mayer, C., Frandsen, P.B., Ware, J., Flouri, T., Beutel, R.G., Niehuis, O., Petersen, M., Izquierdo-Carrasco, F., Wappler, T., Rust, J., Aberer, A.J., Aspöck, U., Aspöck, H., Bartel, D., Blanke, A., Berger, S., Böhm, A., Buckley, T.R., Calcott, B., Chen, J., Friedrich, F., Fukui, M., Fujita, M., Greve, C., Grobe, P., Gu, S., Huang, Y., Jermiin, L.S., Kawahara, A.Y., Krogmann, L., Kubiak, M., Lanfear, R., Letsch, H., Li, Y., Li, Z., Li, J., Lu, H., Machida, R., Mashimo, Y., Kapli, P., McKenna, D.D., Meng, G., Nakagaki, Y., Navarrete-Heredia, J.L., Ott, M., Ou, Y., Pass, G., Podsiadlowski, L., Pohl, H., von Reumont, B.M., Schütte, K., Sekiya, K., Shimizu, S., Slipinski, A., Stamatakis, A., Song, W., Su, X., Szucsich, N.U., Tan, M., Tan, X., Tang, M., Tang, J., Timelthaler, G., Tomizuka, S., Trautwein, M., Tong, X., Uchifune, T., Walzl, M.G., Wiegmann, B.M., Wilbrandt, J., Wipfler, B., Wong, T.K.F., Wu, Q., Wu, G., Xie, Y., Yang, S., Yang, Q., Yeates, D.K., Yoshizawa, K., Zhang, Q., Zhang, R., Zhang, W., Zhang, Y., Zhao, J., Zhou, C., Zhou, L., Ziesmann, T., Zou, S., Li, Y., Xu, X., Zhang, Y., Yang, H., Wang, J., Wang, J., Kjer, K.M. \& Zhou, X. (2014) Phylogenomics resolves the timing and pattern of insect evolution. Science, 346, 763-767.

Moretti, M., Dias, A.T.C., de Bello, F., Altermatt, F., Chown, S.L., Azcarate, F.M., Bell, J.R., Fournier, B., Hedde, M., Hortal, J., Ibanez, S., Öckinger, E., Sousa, J.P., Ellers, J. \& Berg, M.P. (2017) Handbook of protocols for standardized measurement of terrestrial invertebrate functional traits. Functional Ecology, 31, 558-567. 
Ojala, R. \& Huhta, V. (2001) Dispersal of microarthropods in forest soil. Pedobiologia, 45, 443-450.

Pellissier, L., Pradervand, J.-N., Williams, P.H., Litsios, G., Cherix, D. \& Guisan, A. (2013) Phylogenetic relatedness and proboscis length contribute to structuring bumblebee communities in the extremes of abiotic and biotic gradients (ed A Baselga). Global Ecology and Biogeography, 22, 577-585.

Pey, B., Nahmani, J., Auclerc, A., Capowiez, Y., Cluzeau, D., Cortet, J., Decaëns, T., Deharveng, L., Dubs, F., Joimel, S., Briard, C., Grumiaux, F., Laporte, M.-A.A., Pasquet, A., Pelosi, C., Pernin, C., Ponge, J.-F., Salmon, S., Santorufo, L. \& Hedde, M. (2014) Current use of and future needs for soil invertebrate functional traits in community ecology. Basic and Applied Ecology, 15, 194-206.

Pollierer, M.M., Langel, R., Scheu, S. \& Maraun, M. (2009) Compartmentalization of the soil animal food web as indicated by dual analysis of stable isotope ratios $\left({ }^{15} \mathrm{~N} /{ }^{14} \mathrm{~N}\right.$ and $\left.{ }^{13} \mathrm{C} /{ }^{12} \mathrm{C}\right)$. Soil Biology and Biochemistry, 41, 12211226.

Ponge, J.-F., Dubs, F., Gillet, S., Sousa, J. \& Lavelle, P. (2006) Decreased biodiversity in soil springtail communities: the importance of dispersal and landuse history in heterogeneous landscapes. Soil Biology and Biochemistry, 38, 1158-1161.

Potapov, A.A., Semenina, E.E., Korotkevich, A.Y., Kuznetsova, N.A. \& Tiunov, A. V. (2016) Connecting taxonomy and ecology: Trophic niches of collembolans as related to taxonomic identity and life forms. Soil Biology and Biochemistry, 101, 20-31.

Prinzing, A. (2016) On the opportunity of using phylogenetic information to ask evolutionary questions in functional community ecology. Folia Geobotanica, 51, 69-74.

Rosado, B.H.P., Matos, I.S. \& Amorim, T. de A. (2016) A matter of scale and traits: a comment on "On the need for phylogenetic 'corrections' in functional trait-based approaches” by de Bello et al. (2015). Folia Geobotanica, 51, $383-387$.

Rosenfeld, J.A., Payne, A. \& DeSalle, R. (2012) Random roots and lineage sorting. Molecular Phylogenetics and Evolution, 64, 12-20.

Scheu, S. \& Falca, M. (2000) The soil food web of two beech forests (Fagus sylvatica) of contrasting humus type: stable isotope analysis of a macro- and a mesofauna-dominated community. Oecologia, 123, 285-296.

Schneider, C., Cruaud, C. \& D’Haese, C.A. (2011) Unexpected diversity in Neelipleona revealed by molecular phylogeny approach (Hexapoda, Collembola). Soil Organisms, 83, 383-398.

Sha, D., Gao, M., Sun, X., Wu, D. \& Zhang, X. (2015) Relative Contributions of Spatial and Environmental Processes and Biotic Interactions in a Soil Collembolan Community. Chinese Geographical Science, 25, 582-590.

Silvertown, J., McConway, K., Gowing, D., Dodd, M., Fay, M.F., Joseph, J.A. \& Dolphin, K. (2006) Absence of phylogenetic signal in the niche structure of meadow plant communities. Proceedings of the Royal Society B, 273, 3944.

Šímová, I. (2016) Phylogenies are relevant when assessing environmental filtering. Folia Geobotanica, 51, 65-68.

Stötefeld, L., Scheu, S. \& Rohlfs, M. (2012) Fungal chemical defence alters density-dependent foraging behaviour and success in a fungivorous soil arthropod. Ecological Entomology, 37, 323-329.

van Straalen, N.M., Timmermans, M.J.T.N., Roelofs, D. \& Berg, M.P. (2008) Apterygota in the spotlights of ecology, evolution and genomics. European Journal of Soil Biology, 44, 452-457.

Vellend, M. (2010) Conceptual synthesis in community ecology. The Quarterly Review of Biology, 85, 183-206.

Vellend, M. (2016) The Theory of Ecological Communities. Princeton University Press, Princeton.

Widenfalk, L.A., Bengtsson, J., Berggren, Å., Zwiggelaar, K., Spijkman, E., Huyer-Brugman, F. \& Berg, M.P. (2015) Spatially structured environmental filtering of collembolan traits in late successional salt marsh vegetation. Oecologia, 179, 537-549. 
Widenfalk, L.A., Malmström, A., Berg, M.P. \& Bengtsson, J. (2016) Small-scale Collembola community composition in a pine forest soil - Overdispersion in functional traits indicates the importance of species interactions. Soil Biology and Biochemistry, 103, 52-62.

van der Wurff, A.W.G., Isaaks, J.A., Ernsting, G. \& van Straalen, N.M. (2003) Population substructures in the soil invertebrate Orchesella cincta, as revealed by microsatellite and TE-AFLP markers. Molecular Ecology, 12, 1349-1359.

Xiong, Y., Gao, Y., Yin, W. \& Luan, Y. (2008) Molecular phylogeny of Collembola inferred from ribosomal RNA genes. Molecular Phylogenetics and Evolution, 49, 728-735.

Yu, D., Zhang, F., Stevens, M.I., Yan, Q., Liu, M. \& Hu, F. (2016) New insight into the systematics of Tomoceridae (Hexapoda, Collembola) by integrating molecular and morphological evidence. Zoologica Scripta, 45, 286-299.

Zhang, B., Chang, L., Ni, Z., Sun, X. \& Wu, D. (2017) Directional migration of three Desoria species (Collembola: Isotomidae) on the snow surface in late winter. European Journal of Soil Biology, 81, 64-68.

Zhang, B., Chen, T.-W., Mateos, E., Scheu, S. \& Schaefer, I. Cryptic species in Lepidocyrtus lanuginosus (Collembola: Lepidocyrtidae) are sorted by habitat types. Pedobiologia. In revision. 


\section{Acknowledgements}

I owe many thanks to Stefan Scheu for the opportunity to be part of his working group. He was and is the best supervisor with great support in many aspects during my whole $\mathrm{PhD}$ period. His knowledge and enthusiasm inspire me with new ideas and the motivation for going on. I still remember the warmest smile he gave to me at the entrance of the Zoological Institute when the first time I came to Göttingen for the interview.

I thank Holger Kreft for his efforts as referee for this dissertation. I appreciate Mark Maraun, Marko Rohlfs, Elvira Hörandl and Kerstin Wiegand for their willingness and the time they have invested in being part of the committee.

Special thanks to Andrea Lambertz and the technicians in the working group, Christel Fischer, Susanne Böning-Klein, Guido Humpert, Theodora Volovei, Ingrid Kleinhans, Dieter Nünchert, Andrea Graebe and Elisabeth Opielka. Without their efforts and organization, most things could not run smoothly in the labs or the working group.

I would like to thank the colleagues who helped me with field work, Bernhard Eitzinger, Jens Bast, Patrick Pachl, Kathleen Lemanski, Odette Gonzalez, Paul Götsch, Andreas Klein and Charlotte Seifert, and with Collembola identification, Nicole Scheuermann and Xin Sun. I also thank Ina Schaefer for her introductions to molecular phylogenetic analyses, as well as Tamara Hartke and Andrew Davis for their corrections to my writing in English. Special thanks to the people reviewing this dissertation; in addition to Stefan, Tamara and Andrew, this should also include Mark, Ina and Gerrit Moser; their comments on the manuscripts improved the quality of the thesis very much.

Thanks to the core members in the paper writing group, Tamara, Odette and Nico Radermacher; gathering together really speeded up the writing. Also thanks to the participants in the paper/book reading group, Tamara, Odette, Ina, Bing Zhang, Zhipeng Li, Xin Gong, Bastian Heinburger and those I forgot to mention; they have inspired me with lots of ideas and helped me to reformulate my thoughts. I was also glad to talk with Huining Zhang; the casual talk surprisingly led to a project within this thesis (Chapter 5), even though she is an expert in cicada taxonomy.

Thanks to all the Bachelor and Master students and HiWis, Mona Laura Kahlfeld, Victoria Kreipe, Lars Blumenberg, Yu-De Lin, Einar Michael Unger, Denise Castle, Jo-Fan Chao, Philipp Sandmann, Sarah Wehrmaker, Birk Urmersbach and Gerrit M., for their time working with me and all they have accomplished.

I also appreciate the inspiring discussions with Mark, Marko, Andrew, Tamara, Ina, Bing, Xin G., Bernhard E., Anton Potapov, Matty P. Berg, Tancredi Caruso, Stefanie Maaß, Meixiang Gao and Donghui $\mathbf{W u}$, and still remember what we may go on with in the future.

I would like to thank the whole AG Scheu for such a nice working atmosphere. I have really enjoyed the time here. 
What also impresses me a lot is the "German learning lunch" with Jens, Bernhard E. and Alexandre Jousset along the walk to the Mensa as well as our friendships. I received great support from my friends in Göttingen and would like to thank Elissa H. Lee-Drude and her family, Che-Wei Chang, Chao-Chin Chan, Po-Yin Chiang, "Right Fist" Chang, Sheng-An Yang, Sheng-Yen Feng and Wei-An Sheng; they have made life in Germany not lonely and boring but colorful and wonderful. I also thank Liang Chang, Bing, Xin S. and Xin G. for the relaxing talks in our fluent mother tongue. In addition, thanks to the Lord Jesus Christ for the support from the Brothers and Sisters in the Church life in Göttingen.

I am grateful for the scholarship given by the Ministry of Education, Taiwan and the BEST-project funded by the German Ministry of Education, Science and Technology (BMBF).

I would like to thank Ping-Chun Lucy Hou, who was a pioneer in the field, for bringing me to the world of soil invertebrates.

Last, I give my special thanks to my beloved Jo-Fan Chao for her encouragement, patience, company and love in all situations, and to my family in Taiwan for all kinds of their support.

謝謝大家！ 


\section{$\underline{\text { List of publications }}$}

\section{Published in peer-reviewed journals}

Chen, T.-W., P. Sandmann, I. Schaefer and S. Scheu (2017) Neutral lipid fatty acid composition as trait and constraint in Collembola evolution. Ecology and Evolution, 7, 9624-9638.

Talbot, B, T.-W. Chen, S. Zimmerman, S. Joost, A. J. Eckert, T. M. Crow, D. Semizer-Cuming, C. Seshadri, and S. Manel (2017) Combining genotype, phenotype, and environment to infer potential candidate genes. Journal of Heredity, 108, 207-216.

Chen, T.-W., C.-P. Wang, and P.-C. L. Hou (2008) A review on the impacts of the invasive earthworms, Pontoscolex corethrurus, on forest ecosystems. Quarterly Journal of Chinese Forestry, 41, 135-148 (in Chinese with English abstract).

Chang, W.-L., T.-W. Chen, C.-Y Yang, Y.-H Huang, and D. Chao (2004) Prevalence and observation of intestinedwelling gregarines in the millipede Trigoniulus corallinus (Spirobolida: Pachybolidae) collected from Shoushan, Kaohsiung, Taiwan. Formosan Entomologist, 24, 137-145.

\section{Submitted}

Zhang, B.†, T.-W. Chen†, E. Mateos, S. Scheu and I. Schaefer. Cryptic species in Lepidocyrtus lanuginosus (Collembola: Lepidocyrtidae) are sorted by habitat types. Pedobiologia. In revision.

(†These authors contributed equally to this paper.)

Gong, X.t, T.-W. Chent, S. Zieger, C. Bluhm, K. Heidemann, I. Schaefer, M. Maraun, M. Liu and S. Scheu. Phylogenetic and trophic determinants of gut microbiota in soil detritivores. Submitted.

(†These authors contributed equally to this paper.) 


\section{Thesis declarations}

\section{Declaration of the author's own contribution to manuscripts with multiple authors}

Chapter 3 has been published in a peer-reviewed journal; Philipp Sandmann collected the fatty acid data and performed his Bachelor thesis under my supervision. Trait data used in Chapters $\mathbf{2}$ and 4 was provided by Matty P. Berg. Data on molecular sequences in Chapter 2 was obtained with the help of JoFan Chao. Abundance data on Collembola in Chapter 4 was collected with the help of Jo-Fan Chao; Victoria Kreipe performed her Master thesis under my supervision which was related to Chapter 4. Community data used in Chapter $\mathbf{5}$ was kindly provided by Apolline Auclerc, Jean-François Ponge, Sébastien Barot and Florence Dubs; Gerrit Moser conducted the preliminary analyses for the data and performed his Master thesis under my supervision.

I am the first author of all chapters; I have developed the main ideas, designed the studies, analyzed the data, written the manuscripts and created tables, figures and supplementary materials. 


\section{Plagiarism declaration}

I, Ting-Wen Chen, declare that I have written this doctoral thesis independently. All persons contributing to the manuscripts have been named so. All sentences or passages quoted from other people's work have been specifically acknowledged by clear cross-referencing.

I have not submitted this thesis in any form for another degree at any university or institution.

Ting-Wen Chen

Göttingen, December 2017 
